\title{
Comparative Analysis of Inactivated Wood Surfaces
}

\author{
Milan Sernek \\ Dissertation submitted to the Faculty of the \\ Virginia Polytechnic Institute and State University \\ in partial fulfillment of the requirements for the degree of \\ Doctor of Philosophy \\ in \\ Wood Science and Forest Products
}

Dr. Wolfgang G. Glasser, Co-chair

Dr. Frederick A. Kamke, Co-chair

Dr. John G. Dillard

Dr. Charles E. Frazier

Dr. Richard F. Helm

April 24, 2002

Blacksburg, Virginia

Keywords: Wood Surface Inactivation, XPS, Wettability, Adhesion,

Fracture Mechanics, Extractives

Copyright 2002, Milan Sernek 


\title{
Comparative Analysis of Inactivated Wood Surfaces
}

\author{
Milan Sernek \\ Wolfgang G. Glasser and Frederick A. Kamke, Co-Chairs
}

\begin{abstract}
(ABSTRACT)
A wood surface, which is exposed to a high temperature condition, can experience inactivation. Surface inactivation reflects physical and chemical modifications of the wood surface. Consequently, these changes result in reduced ability of an adhesive to properly wet, flow, penetrate, and cure. Thus, an inactivated wood surface does not bond well with adhesives.

The changes in surface chemistry, wettability, and adhesion of inactivated wood surfaces, including heartwood of yellow-poplar (Liriodendron tulipifera) and southern pine (Pinus taeda), were studied. Wood samples were dried from the green moisture content condition in a convection oven at five different temperature levels ranging from 50 to $200{ }^{\circ} \mathrm{C}$. The comparative characterization of the surface was done by X-ray photoelectron spectroscopy (XPS), sessile drop wettability, and fracture testing of adhesive bonds. Additionally, several chemical treatments were utilized to improve wettability and adhesion of inactivated wood surfaces.

The comparative analysis helped elucidate clear relationships between surface chemistry, wettability, and bond performance in regard to surface inactivation. XPS results showed that wood drying caused modification in wood surface chemistry. The oxygen to carbon ratio $(\mathrm{O} / \mathrm{C})$ decreased and the $\mathrm{C} 1 / \mathrm{C} 2$ ratio increased with drying temperature. The $\mathrm{C} 1$ component is related to carbon-carbon or carbon-hydrogen bonds, and the $\mathrm{C} 2$ component represents single carbonoxygen bond. A low $\mathrm{O} / \mathrm{C}$ ratio and a high $\mathrm{C} 1 / \mathrm{C} 2$ ratio reflected a high concentration of non-polar wood components (extractives/VOCs) on the wood surface, which modified the wood surface from hydrophilic to more hydrophobic. A hydrophobic wood surface repelled water and wettability of this surface was low (i.e., a high contact angle). Wettability was directly related to the $\mathrm{O} / \mathrm{C}$ ratio and inversely related to the $\mathrm{C} 1 / \mathrm{C} 2$ ratio.
\end{abstract}


Contact angle decreased with time and increased with the temperature of exposure. A dependence of wood species was evident. Southern pine had a lower wettability than yellowpoplar, which was due to a greater concentration of non-polar hydrocarbon-type extractives and heat-generated volatiles on the surface. Solvent extraction prior to drying did not improved wettability, whereas, extraction after drying improved wettability. A contribution of extractives migration and pyrolysis products deposition played a significant role in the heat-induced inactivation process of southern pine.

The maximum strain energy release rate $\left(\mathrm{G}_{\max }\right)$ obtained by fracture testing showed that surface inactivation was insignificant for yellow-poplar when exposed to drying temperatures < $187^{\circ} \mathrm{C}$. The southern pine was most susceptible to inactivation particularly when bonded with phenol-formaldehyde (PF) adhesive. A typical surface inactivation for southern pine occurred at drying temperatures $>156^{\circ} \mathrm{C}$.

Chemical treatments improved the wettability of inactivated wood surfaces, but an improvement in adhesion was not evident for specimens bonded with polyvinyl-acetate (PVA) adhesive. Of the chemical treatments employed in this study, $\mathrm{NaOH}$ was most effective for improving adhesion of the PF adhesive bond. $\mathrm{G}_{\max }$ of southern pine specimens treated with $\mathrm{NaOH}$ increased by a factor of three compared with inactivated specimens. Enzymatic treatment of inactivated surfaces with xylanases did not improve adhesion and this ruled out temperatureinduced hornification of fibers as being responsible for surface inactivation. Bonding of inactivated southern pine with a polyisocyanate adhesive significantly improved the adhesive bond performance. However, this improvement reached $<70 \%$ of the adhesion established between freshly produced wood surfaces bonded with PVA or PF adhesives. 


\section{Dedication}

To my wife Iris, daughter Barbara, and son Matija.

They are my love, my joy, my pride, ... my everything. 


\section{Acknowledgements}

I would like to express my sincere thanks and appreciation to my advisors, Dr. Wolfgang G. Glasser and Dr. Frederick A. Kamke, for their guidance, tutoring, and encouragement during my graduate study. Dr. Glasser inspired me with his ideas and enriched my knowledge with fruitful discussions. Dr. Kamke directed my research, motivated me with challenges, and elucidated to me many aspects of wood-based composites. They were excellent committee cochairs. I also thank Dr. Kamke for inviting me to Virginal Tech in 1997 as a visiting scientist.

I would also like to express my sincere gratitude to Dr. John G. Dillard, Dr. Charles E. Frazier, and Dr. Richard F. Helm, members of my committee for their discussions, comments, and advices. I would like to thank former department head Dr. Geza Ifju and current department head Dr. Paul Winistorfer for their help during my graduate study.

I would like to acknowledge the effort and help of my former advisor, Dr. Joze Resnik, at the University of Ljubljana, Department of Wood Science, who introduced me to Dr. Kamke. Thanks to Dr. Niko Torelli for his contribution to my study.

I thank Kenneth Albert, Robert Carner, Frank Cromer, Carlisle Price, Harrison Sizemore, and Robert Wright for their assistance in my research. I appreciate the administrative help from Joanne Buckner, Linda Caudill, Sharon Daley, Debra Garnand, and Angie Riegel. I thank the tutors at the Virginia Tech Writing Center, who helped me with my English writing. Thanks to the students and all the others at the Department of Wood Science and Forest Products.

I greatly appreciate the financial support of this research from The Wood-Based Composite Center at Virginia Tech. I would like to thank the National Starch and Chemical Co. and the Dynea Co. for adhesives supplies. I greatly appreciate the financial contribution of the Slovenian Ministry of Education, Science and Sport, and its allowance for switching my study in Slovenia to study in the U.S.

Thanks to my mother and especially to my father who inspired and motivated me with a constructive criticism of my achievements. Last, but by no means least, I would like to thank my wife, Iris, for her love, trust, understanding, support, and help. 
This dissertation comprises seven chapters. Chapter 1 introduces the subject of the study, defines the problem, exposes the postulations, and states the objectives. Chapter 2 reviews wood surface inactivation phenomenon, explains principles of two analytical methods employed in wood surface characterization (XPS and contact angle), and describes the fracture mechanics approach for evaluation of wood adhesion. Chapters 3, 4, 5, and 6 present the experimental studies. Chapter 3 interprets the consequences of thermal inactivation on the chemistry and wettability of a wood surface. The second part of the chapter provides temperature dependence data of wood inactivation for two wood species, and it evaluates adhesion in regard to inactivation. This chapter also establishes relationships among surface chemistry, wettability, and adhesion of inactivated wood surfaces. Chapter 4 summarizes the theoretical aspect of wood surface chemistry, and then evaluates the surface chemistry of several wood components experimentally. This chapter also elucidates the possible mechanisms involved in wood surface inactivation. Chapter 5 focuses on the inactivation study of one wood species only-the most susceptible one. Several surface treatments and adhesive modifications examine possible remedies for weak adhesion of inactivated surfaces. Chapter 6 uses knowledge gained from previous experimental work to introduce a reliable method for the fast detection of wood surface inactivation. Finally, Chapter 7 summarizes the findings and draws the conclusions from all conducted studies on thermally inactivated wood surfaces. 


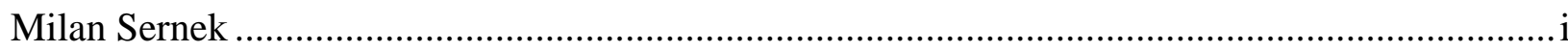

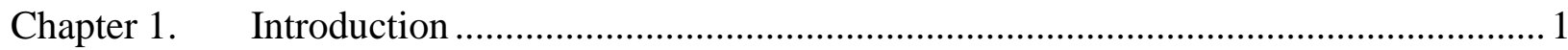

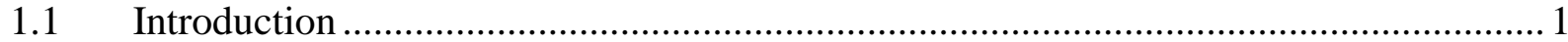

1.2 Problem Definition and Research Justification ........................................................... 1

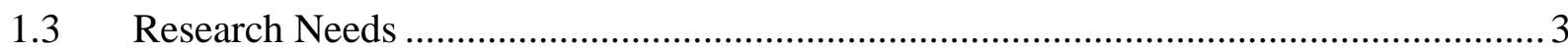

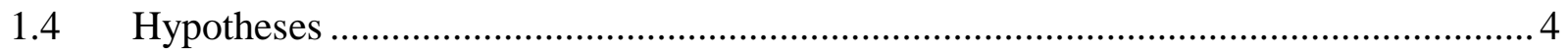

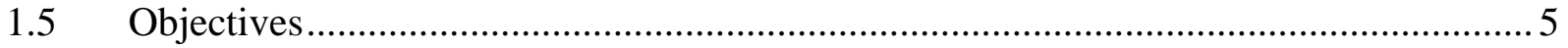

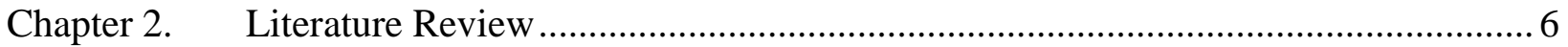

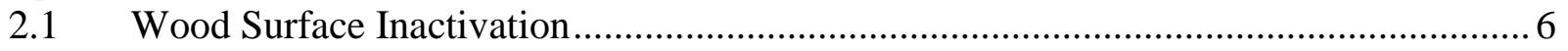

2.2 Sources and Causes of Wood Inactivation ..................................................................... 7

2.3 Factors Affecting Wood Surface Inactivation............................................................ 8

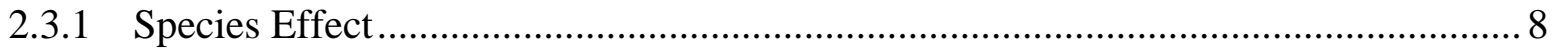

2.3.2 Effect of High Temperature and Time ……….................................................... 9

2.3.3 Effect of Drying Technique............................................................................... 11

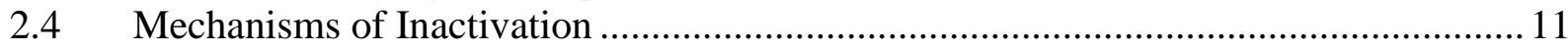

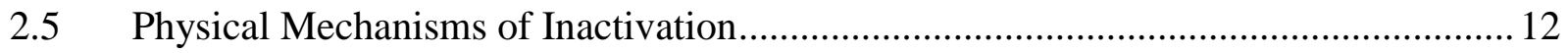

2.5.1 Effect of Extractives on Wettability and Adhesion................................................... 12

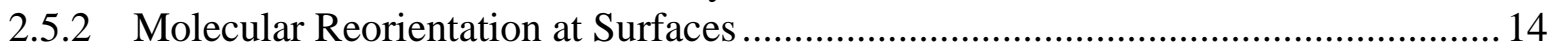

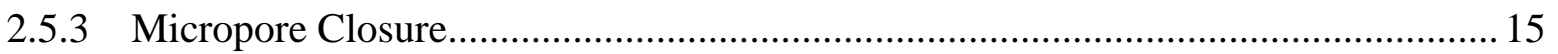

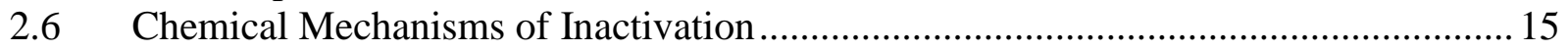

2.6.1 Elimination of Surface Hydroxyl Bonding Sites.................................................... 15

2.6.2 Oxidation and/or Pyrolysis of Surface Bonding Sites............................................ 16

2.6.3 Chemical Interference with Resin Cure or Bonding ............................................. 16

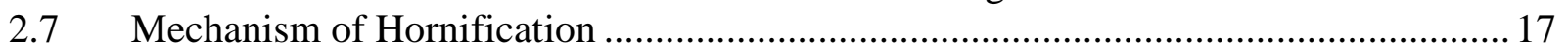

2.8 Measures for Inhibiting Inactivation of Wood Surface................................................ 17

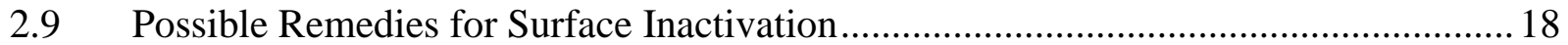

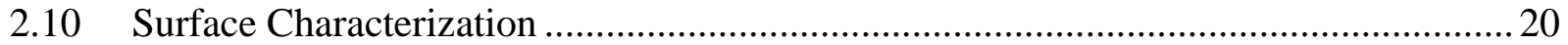

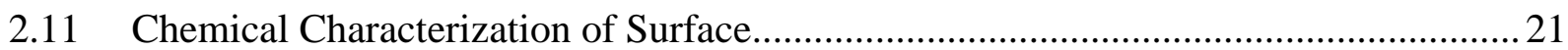

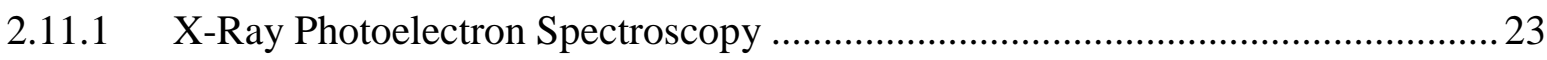

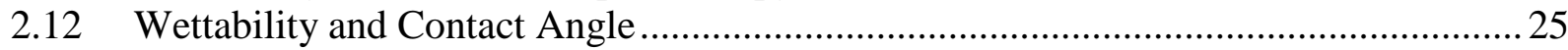

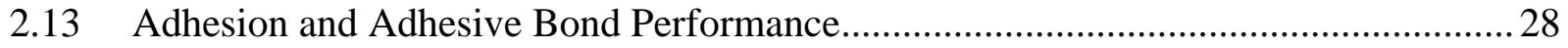

2.13.1.1 Shear Corrected Compliance Method …………............................................... 30

Chapter 3. Characterization of Thermally Inactivated Wood Surfaces ...................................... 32

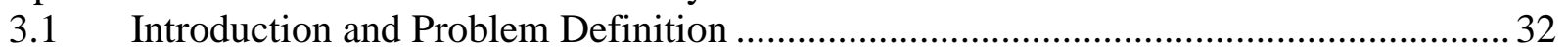

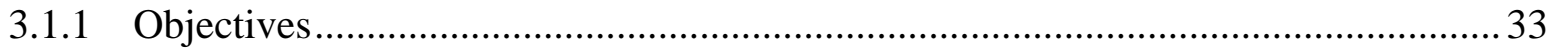

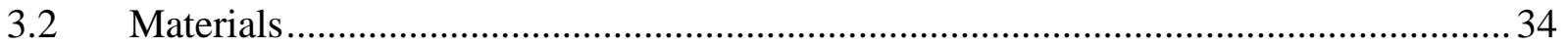

3.2.1 Heat Treatment - Drying of Wood Samples ……................................................... 34

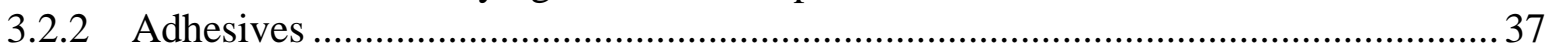

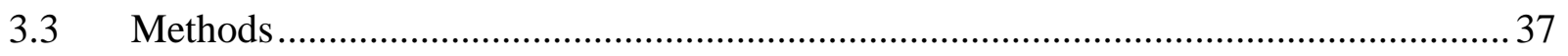

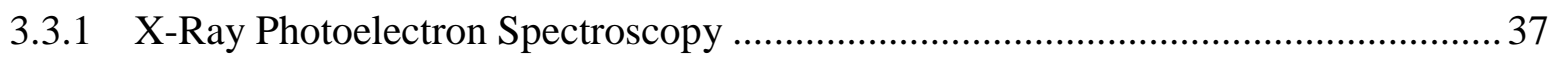

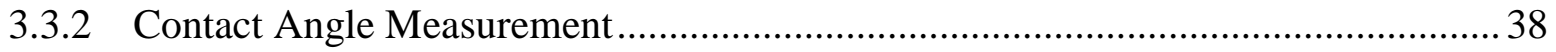




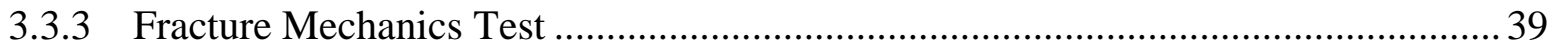

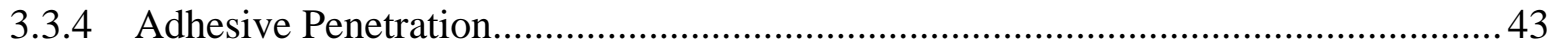

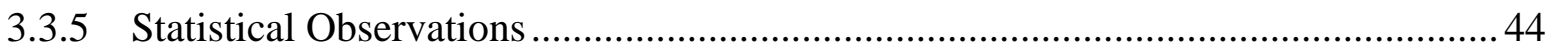

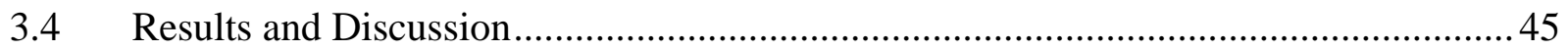

3.4.1 Influence of Drying Temperature on Chemical Changes of Wood Surface ............. 45

3.4.2 Influence of Drying Temperature on Wood Wettability ...........................................5 53

3.4.3 Influence of Drying Temperature on Adhesive Bond Performance ..........................59

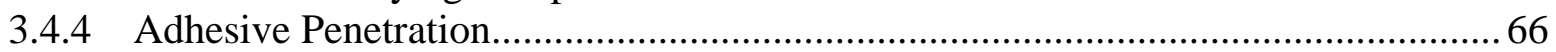

3.4.5 Relationships among Wood Surface Chemistry, Wettability, and Adhesion ............ 68

3.4.5.1 Wettability and Chemical Composition ...................................................................6 68

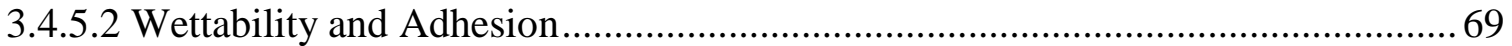

3.4.5.3 Chemical Composition and Adhesion .............................................................. 72

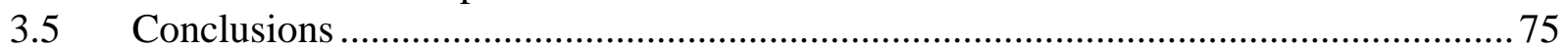

Chapter 4. Wood Surface Chemistry, Wettability, and Adhesion ............................................. 76

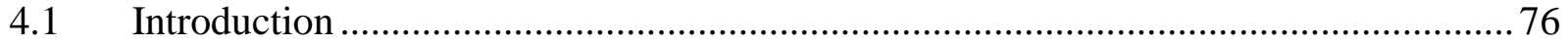

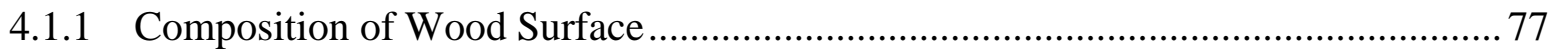

4.1.2 The O/C Ratio and the C1/C2 Ratio of Cellulose and Hemicelluloses..................... 78

4.1.3 The O/C Ratio and the C1/C2 Ratio of Lignin..................................................... 79

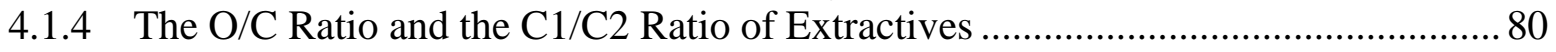

4.1.4.1 Extractives of Yellow-Poplar ............................................................................ 80

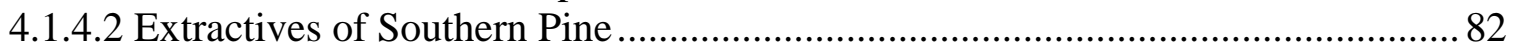

4.1.4.3 Hydrocarbon and Carbohydrate Types of Extractives .......................................... 84

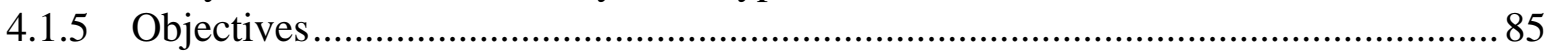

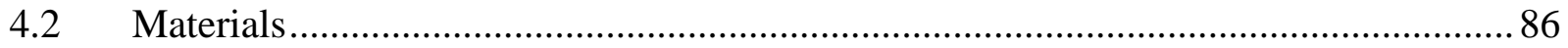

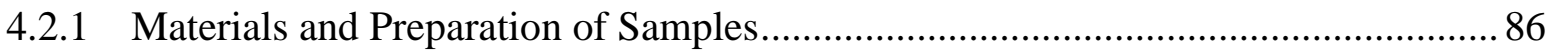

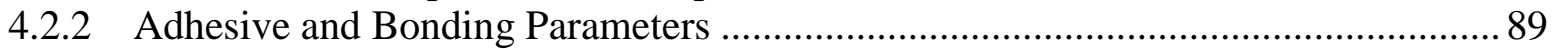

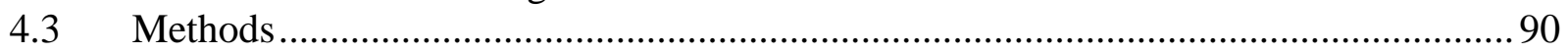

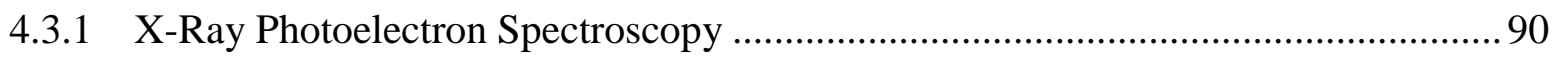

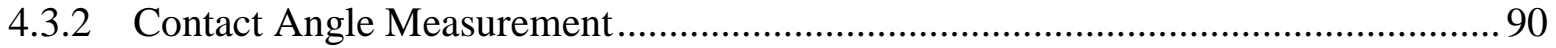

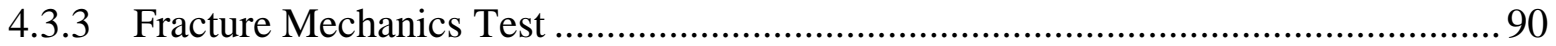

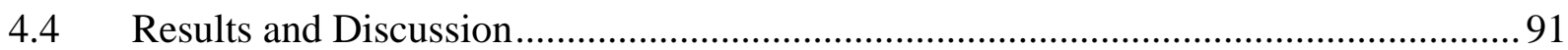

4.4.1 Chemical Characterization of Wood Surfaces ......................................................91

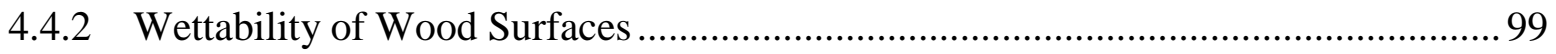

4.4.2.1 Relationship between Wood Surface Chemistry and Wettability ....................... 102

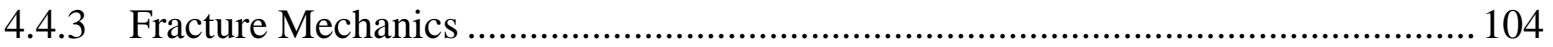

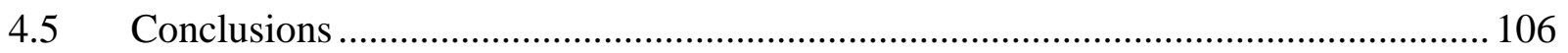

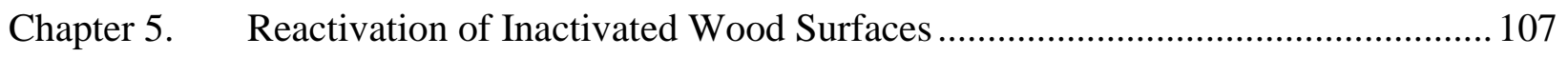

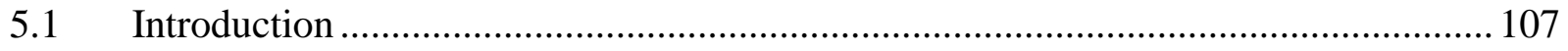

5.1.1 Hydroxymethylated Resorcinol....................................................................... 108

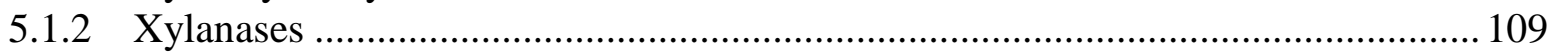

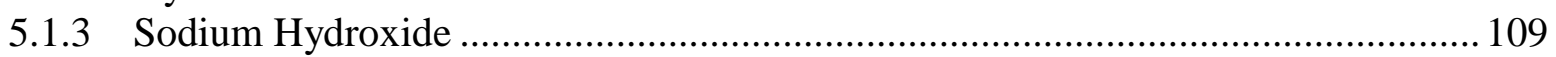

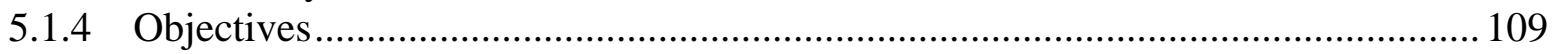

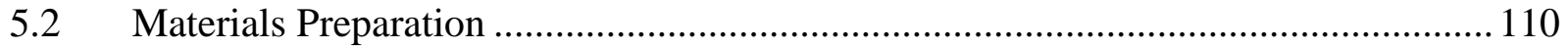

5.2.1 Drying of Wood Samples................................................................................. 110 


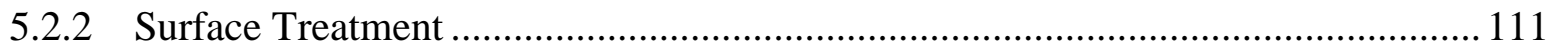

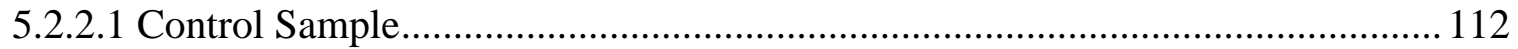

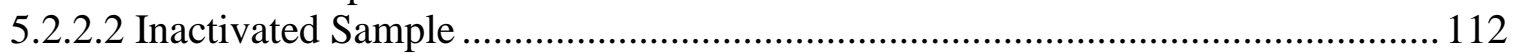

5.2.2.3 Inactivated Sample Treated with Hydroxymethylated Resorcinol ...................... 112

5.2.2.4 Inactivated Sample Treated with Enzyme Xylanase............................................ 113

5.2.2.5 Inactivated Sample Treated with Sodium Hydroxide ………………………....... 113

5.2.2.6 Inactivated Sample Treated with Xylanase and Sodium Hydroxide.................... 114

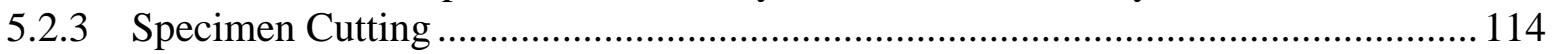

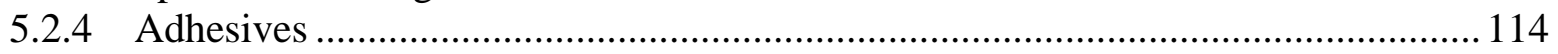

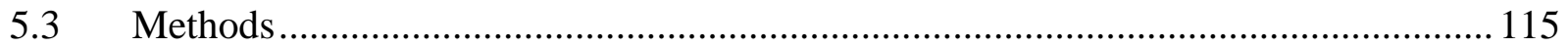

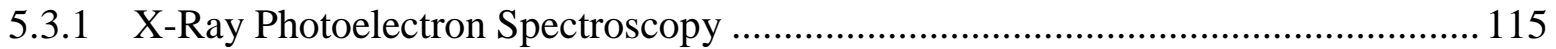

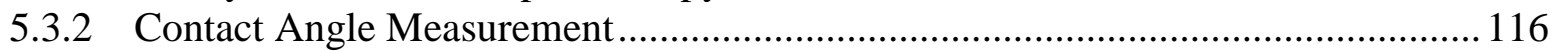

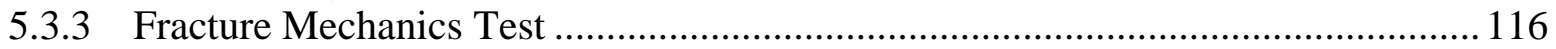

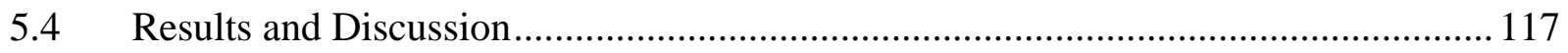

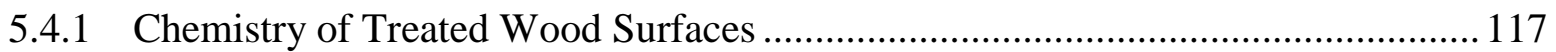

5.4.2 Effect of Surface Treatment on Wettability of Southern Pine .................................. 118

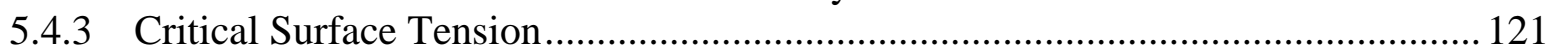

5.4.4 Effect of Surface Treatment on Adhesion........................................................... 125

5.4.4.1 Specimens Bonded with PVA Adhesive ………………................................. 125

5.4.4.2 Specimens Bonded with PF Adhesive …………............................................ 128

5.4.4.3 Effect of Adhesive on $\mathrm{G}_{\max }$ of Inactivated Specimens....................................... 132

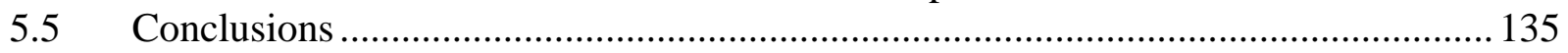

Chapter 6. Method for Detection of Wood Surface Inactivation........................................... 136

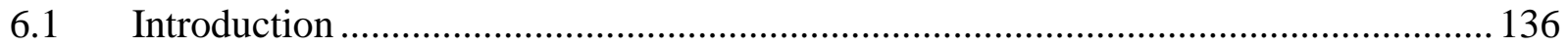

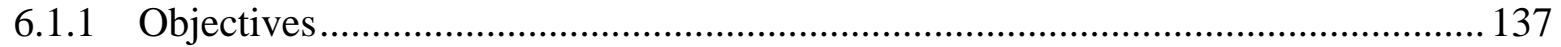

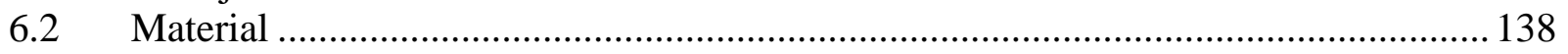

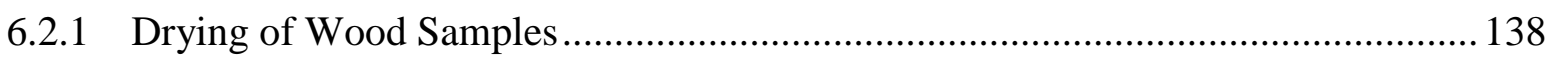

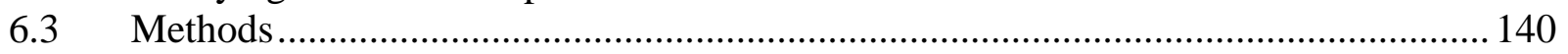

6.3.1 Surface Inactivation Method ......................................................................... 140

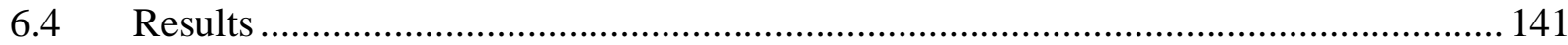

6.4.1 Wood Surface Inactivation Tested with PF Adhesive ............................................. 141

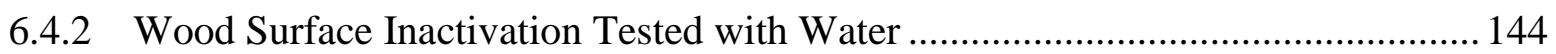

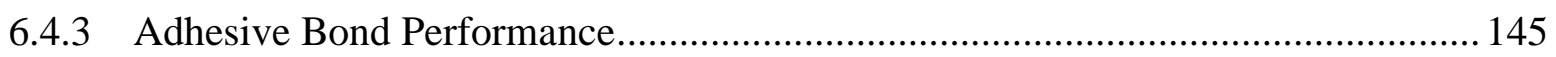

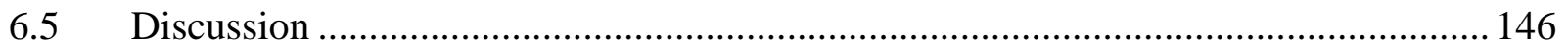

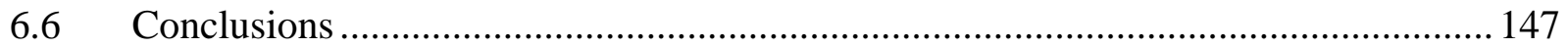

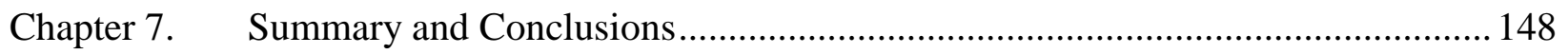

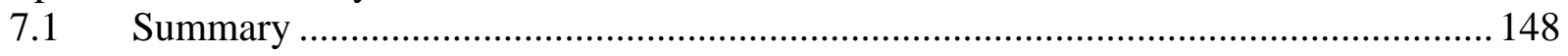

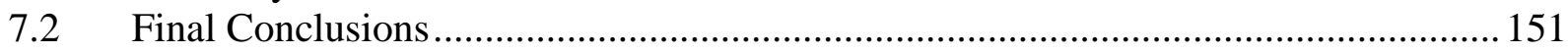

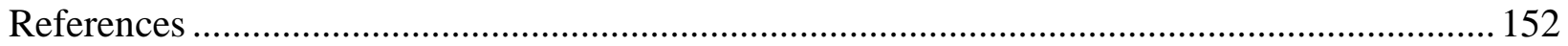

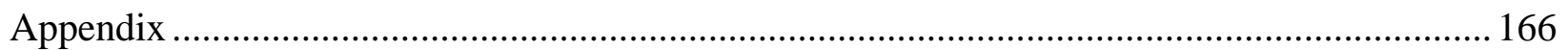

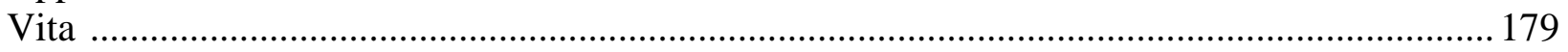




\section{List of Figures}

Figure 2.1. Influence of thermal treatment on Fir wettability (Podgorski et al. 2000)............... 10 Figure 2.2. Influence of the plasma treatment time on fir wettability (Podgorski et al. 2000).... 19 Figure 2.3. Lignin deposition on fiber surfaces after kraft pulping (Li and Reeve 2000)........... 20 Figure 2.4. The regimes of surface analysis, thin film analysis and bulk analysis

(Briggs and Seah 1990).................................................................................. 21

Figure 2.5. Escape characteristic of photoelectrons in XPS. ................................................ 24

Figure 2.6. Contact angle and interfacial surface tensions at equilibrium...............................25

Figure 2.7. Critical surface tension plot (Schrader and Loeb 1992)...................................... 27

Figure 3.1. The machining of the wood samples: timber (left), lamellae (right)........................34

Figure 3.2. The increase of the wood surface temperature during drying. ................................. 35

Figure 3.3. VOCs emission from dried particle at various temperatures

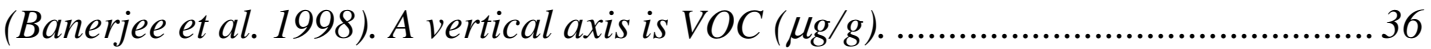

Figure 3.4. Specimen cutting diagram for each lamella. Width $(\mathrm{mm})$ is tangential direction. .... 36

Figure 3.5. The contact angle equipment set-up................................................................... 39

Figure 3.6. Geometry and dimensions $(\mathrm{mm})$ of the fracture test specimen. ............................... 40

Figure 3.7. Fracture test setup showing a mounted specimen and the specimen grip............... 40

Figure 3.8. TestWorks ${ }^{T M}$ data acquisition system with the parameters setup for fracture test.... 42

Figure 3.9. Measurement parameters used in calculating EP and MP ................................... 44

Figure 3.10. Wide scan XPS spectrum for southern pine surface exposed to $200^{\circ} \mathrm{C}$.................. 45

Figure 3.11. Curve fits of carbon C1s peak of southern pine surface exposed to $200{ }^{\circ} \mathrm{C}$............. 46

Figure 3.12. Curve fits of O1s peak of southern pine surface exposed to $200^{\circ} \mathrm{C}$........................ 47

Figure 3.13. The influence of drying temperature on the O/C atomic ratio of yellow-poplar. .... 49

Figure 3.14. The influence of drying temperature on the O/C atomic ratio of southern pine..... 49

Figure 3.15. The influence of drying temperature on the $C 1 / C 2$ atomic ratio of yellow-poplar. ................................................................................................ 51

Figure 3.16. The influence of drying temperature on the $C 1 / C 2$ atomic ratio of southern pine ............................................................................................................ 51

Figure 3.17. Typical initial contact angle of a water drop on the SP wood surface dried at $51^{\circ} \mathrm{C}$ (left), and on the inactivated SP wood surface dried at $187^{\circ} \mathrm{C}$ (right)....... 54

Figure 3.18. Time dependence of the contact angle for yellow-poplar. .....................................55

Figure 3.19. Time dependence of the contact angle for southern pine....................................... 55

Figure 3.20. The rate of contact angle change during one minute in respect to drying

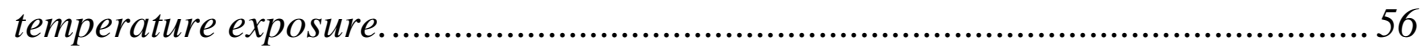

Figure 3.21. The rate of contact angle decline for yellow-poplar............................................5 58

Figure 3.22. The rate of contact angle decline for southern pine ..............................................5 58

Figure 3.23. A typical load-displacement curve obtained from DCB by fracture testing. .......... 59

Figure 3.24. A typical plot of the cube root of compliance versus crack length........................ 60

Figure 3.25. A typical plot of SERR versus crack length for a single DCB specimen................. 60

Figure 3.26. Influence of drying temperature on the maximum strain energy release rate of yellow-poplar adhesive bond. 
Figure 3.27. Influence of drying temperature on the maximum strain energy release rate of southern pine adhesive bond.

Figure 3.28. PF adhesive bond failure in regard to drying temperature exposure:

$Y P$ dried at $51^{\circ} \mathrm{C}$ (left), and YP dried at $187^{\circ} \mathrm{C}$ (right).....

Figure 3.29. PF adhesive bond failure in regard to drying temperature exposure:

SP dried at $51^{\circ} \mathrm{C}$ (left), and SP dried at $187^{\circ} \mathrm{C}$ (right).

Figure 3.30. A poor adhesive bond (left) caused by extensive deposition of extractives on the SP surface (right).

Figure 3.31. PF adhesive penetration into YP exposed to $51^{\circ} \mathrm{C}$ (left) and $187^{\circ} \mathrm{C}$ (right). 67

Figure 3.32. PF adhesive penetration into SP exposed to $51^{\circ} \mathrm{C}$ (left) and $187^{\circ} \mathrm{C}$ (right). 67

Figure 3.33. Relationship between initial wettability of $Y P$ and $S P$ and the $O / C$ ratio...... 68

Figure 3.34. Relationship between initial wettability of YP and SP and the C1/C2 ratio........... 69

Figure 3.35. Relationship between adhesion and wettability for YP and SP bonded with PF. 70

Figure 3.36. Relationship between adhesion and wettability for YP and SP bonded with PVA. 70

Figure 3.37. Relationship between adhesion and rate of contact angle change for SP samples bonded with PF adhesive. 71

Figure 3.38. Relationship between adhesion and rate of contact angle change for YP samples bonded with PF adhesive. ............................................................. 72

Figure 3.39. Relationship between adhesion and $\mathrm{O} / \mathrm{C}$ ratio for $Y \mathrm{P}$ and $S P$.............................. 73

Figure 3.40. Relationship between adhesion and $C 1 / C 2$ ratio for $Y P$ and $S P$......................... 73

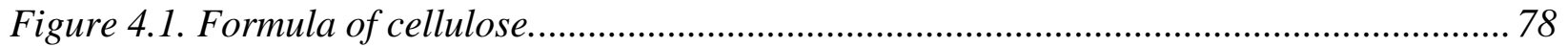

Figure 4.2. Lignin precursors: p-coumaryl (I), coniferyl (II), and sinapyl (III) alcohols........... 79

Figure 4.3. Yellow-poplar alkaloid liriodenine (left), and lignan syringaresinol (right)............ 81

Figure 4.4. Common monoterpenes of southern pine ......................................................... 82

Figure 4.5. Typical resin acids in southern pine: abietic (left) and pimaric (right).................. 83

Figure 4.6. Typical increase of wood surface temperature during drying. ............................... 88

Figure 4.7. Extraction of the wood samples in a big Soxhlet extractor..................................... 89

Figure 4.8. Orientation, geometry and dimensions $(\mathrm{mm})$ of the fracture test specimen.............. 90

Figure 4.9. O/C ratio of wood and wood constituents............................................................ 93

Figure 4.10. C1/C2 ratio of wood and wood constituents. .................................................... 95

Figure 4.11. Schematic presentation of curve fit for C1s peak of wood constituents.................. 96

Figure 4.12. Curve fit of C1s peak of XPS spectra for YP (right) and SP (left)....................... 97

Figure 4.13. Initial water contact angle in regard to wood surface treatment......................... 100

Figure 4.14. Time dependence of contact angle for yellow-poplar....................................... 101

Figure 4.15. Time dependence of contact angle for southern pine .......................................... 102

Figure 4.16. Relationship between wettability and the $O / C$ ratio of $Y P$ and $S P$ surfaces......... 103

Figure 4.17. Relationship between wettability and the C1/C2 ratio of YP and SP surfaces..... 103

Figure 4.18. Influence of surface treatment on the SERR of PF adhesive bond....................... 104

Figure 5.1. Resorcinol (left) and trihydroxymethyl resorcinol (right)................................... 108

Figure 5.2. Changes in temperatures during wood drying - a typical plot .............................. 111

Figure 5.3. Specimen cutting diagram for each lamella. Width $(\mathrm{mm})$ is tangential direction. .. 114

Figure 5.4. Orientation, geometry and dimensions $(\mathrm{mm})$ of the fracture test specimen............ 116 
Figure 5.5. Influence of surface treatment on initial water contact angle............................... 118

Figure 5.6. Influence of time and surface treatment on the contact angle of a water drop....... 119

Figure 5.7. The relative change in the contact angle during one minute ................................ 122

Figure 5.8. Critical surface tension plot for inactivated wood surface ..................................... 123

Figure 5.9. Critical surface tension plot for inactivated wood surface treated with xylanase. 124

Figure 5.10. Effect of southern pine surface treatment on SERR of PVA adhesive................... 126

Figure 5.11. Relationship between PVA adhesion and water wettability................................. 127

Figure 5.12. Effect of southern pine surface treatment on SERR of PF adhesive................... 129

Figure 5.13. Relationship between PF adhesion and wood surface wettability........................ 131

Figure 5.14. Effect of adhesive mixture on SERR of bonded SPI specimens.......................... 133

Figure 6.1. Changes in temperatures during southern pine lamellas drying.......................... 139

Figure 6.2. Specimen cutting diagram for each lamella. Width $(\mathrm{mm})$ is tangential direction. .. 139

Figure 6.3. Actual image of a PF adhesive drop (left) and a side view area (right)................ 141

Figure 6.4. PF adhesive and water were used as test liquids to evaluate SP surface inactivation by using inactivation ratio and absorption index. The greater the deviation (i.e., $\triangle I R$ or $\triangle A B I$ ) from 1, the more severe the surface inactivation.... 147 
Table 2.1. Common acronyms for surface analysis techniques (Brune et al. 1997)................... 22

Table 2.2. Survey of the popular techniques for surface analysis (Briggs and Seah 1990) ......... 22

Table 3.1. Properties of wood samples and drying parameters. ............................................ 34

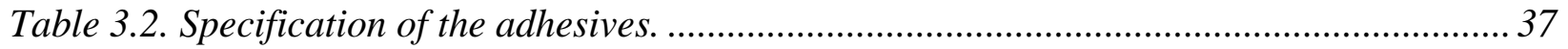

Table 3.3. Atomic percents of yellow-poplar surfaces determined by XPS. .............................. 47

Table 3.4. Atomic percents of southern pine surface as determined by XPS............................. 48

Table 3.5. Average strain energy release rate $\left(\mathrm{J} / \mathrm{m}^{2}\right)$ for yellow-poplar adhesive bond............. 61

Table 3.6. Average strain energy release rate $\left(\mathrm{J} / \mathrm{m}^{2}\right)$ for southern pine adhesive bond................ 61

Table 3.7. Phenol-formaldehyde adhesive penetration into wood............................................ 66

Table 4.1. Possible components of C1s peak of wood, type of bonding, and binding energy. .... 78

Table 4.2. Classification of the extractives with examples according to analysis groups

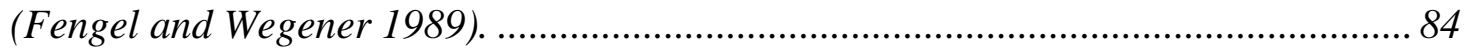

Table 4.3. Type and properties of relevant yellow-polar and southern pine extractives............. 85

Table 4.4. Preparation of wood constituents. ........................................................................... 86

Table 4.5. Treatments of wood samples. ................................................................................. 87

Table 4.6. Atomic percent of wood and wood constituents as determined by XPS.................... 91

Table 4.7. O/C and C1/C2 ratios of wood constituents and wood surfaces. .............................. 92

Table 4.8. Surface coverage by extractives and VOCs in regard to wood species and

drying temperature. ............................................................................................... 94

Table 5.1. Properties of Wood Samples and Drying Parameters. ........................................... 110

Table 5.2. Treatment of the samples for surface reactivation.................................................. 111

Table 5.3. Ingredients for the HMR coupling agent. ........................................................ 112

Table 5.4. Specifications of the adhesive mixtures and curing parameters............................. 115

Table 5.5. Surface tension of liquid probes........................................................................... 116

Table 5.6. Atomic percent of treated southern pine surfaces................................................. 117

Table 5.7. Elemental components of southern pine surface as determined by XPS................. 117

Table 5.8. Contact angle (degree) on treated wood surfaces as a function of time and treatment. Data is an average of 12 measurements............................................ 120

Table 5.9. Relationship between surface tensions of probe liquids and $\theta_{i}$............................. 123

Table 5.10. Statistically significant differences in $G_{\text {max }}$ of PVA adhesive among surface treatments (denoted with *)........................................................................... 125

Table 5.11. Statistically significant differences in $G_{\max }$ of $P F$ adhesive among surface treatments (denoted with *).......................................................................... 128

Table 5.12. Statistically significant differences in $G_{\text {max }}$ of inactivated SP surface among adhesives (denoted with *)........................................................................................ 132

Table 6.1. Properties of wood samples and drying parameters. ............................................... 138

Table 6.2. Contact angle and absorption results for phenol-formaldehyde adhesive. .............. 143

Table 6.3. Two-Sample analysis results (t-test). ............................................................. 144

Table 6.4. Contact angle and absorption of water............................................................... 145

Table 6.5. SERR $\left(\mathrm{J}^{2} \mathrm{~m}^{2}\right)$ of samples bonded with PF adhesive ............................................. 145 


\section{List of Appendices}

Appendix A. X-ray photoelectron spectroscopy results for wood surfaces................................ 166

Appendix B. Water contact angles of yellow-poplar............................................................ 167

Appendix $C$. Water contact angles of southern pine .......................................................... 168

Appendix D. Strain energy release rate results for yellow-poplar specimens bonded with polyvinyl-acetate (PVA) and phenol-formaldehyde $(P F)$ adhesives.................... 169

Appendix E. Strain energy release rate results for southern pine specimens bonded with polyvinyl-acetate (PVA) and phenol-formaldehyde (PF) adhesives.................... 170

Appendix F. Effective and maximum penetration of phenol-formaldehyde adhesive................ 171

Appendix $G$. Water contact angles of extracted and unextracted yellow-poplar samples......... 172

Appendix H. Water contact angle of extracted and unextracted southern pine samples........... 173

Appendix I. Strain energy release rate results of yellow-poplar and southern pine specimens bonded with phenol-formaldehyde adhesive .................................... 174

Appendix J. Water contact angles of treated southern pine surfaces. ...................................... 175

Appendix K. Water contact angles of treated southern pine surfaces. ..................................... 176

Appendix L. Strain energy release rate of treated southern pine bonded with PF, PVA,

PFHMR, and PMDI adhesives.

Appendix M. Strain energy release rate of treated southern pine bonded with PF and PVA adhesives. 


\section{Chapter 1. Introduction}

\subsection{Introduction}

A wood surface, which is exposed to a high temperature condition, can experience surface inactivation. An inactivated wood surface does not bond well with adhesive, because the inactivation process reduces the ability of an adhesive to properly wet, flow, penetrate, and cure. Thus, the ability to establish intimate contact between molecules of wood and adhesive is diminished. Subsequently, the adhesion attractive forces are weak and rare.

Wood inactivation is a surface phenomenon, affecting just a thin outer layer of wood. Removal of the wood surface layer eliminates the inactivation-bonding problem. Initially, Northcott et al. (1959) designated inactivation as "casehardening" of a wood surface. Later, Hancock (1963) introduced the term "inactivated" to denote the apparent adverse effect of this type of wood surface on the reactivity of phenolic adhesive. Troughton and Chow (1971) defined a surface inactivation phenomenon as a heat-induced change in the wood veneer surface resulting in a loss of bonding ability.

\subsection{Problem Definition and Research Justification}

Inactivation reflects physical and chemical modifications of the wood surface. These modifications create hydrophobic and weak boundary layers, which reduce wettability and cause poor adhesion. An adhesive bond assembled from inactivated wood fails at low stress. Bonded inactivated wood fails adhesively rather than cohesively. If any cohesive failure occurs, it will most frequently be the adhesive that fails and not the wood.

Industrial gluing problems, which originate from wood surface inactivation, became apparent in the 1960s. At that time, a fast drying method-such as direct-fired veneer dryersgained popularity because plywood production expanded drastically (Christiansen 1990). Consequently, average drying temperature increased significantly causing many bonding difficulties and excessive adhesive bond failures. 
Harmful inactivation intensifies with increasing temperature. Since temperatures for drying veneers, wood flakes, and wood particles can be very high-up to $400^{\circ} \mathrm{C}$ at the beginning, and around $200^{\circ} \mathrm{C}$ near the end of the drying process-inactivation is often unavoidable in these drying operations. High inlet and outlet drying temperatures are necessary for efficient and economical drying. Besides drying, hot pressing of wood-based composites requires high temperature. The curing process of phenolic adhesive in plywood or oriented strand board (OSB) usually takes place at $200^{\circ} \mathrm{C}$. Thus, the composite surface that is in contact with a hot metal press platen can experience surface inactivation. But wood-based composites are commonly calibrated to the final thickness by sanding of the surfaces, which removes the inactivated layer. On the contrary, inactivated surfaces of veneer, flakes, or particles are not removed after drying. Hence, most of the inactivation problems are associated with high temperature drying.

Drying is an inevitable process in the wood-based composite industry. High moisture content (MC) of a green wood material has to be reduced prior to manufacturing. Otherwise, high water vapor pressure can blow a composite apart during the opening of a hot press. Moreover, shrinkage that occurs in wet wood generates internal stresses in the wood-adhesive interface, and the adhesive bond can fail. Thus, a proper MC is one of the preconditions for achieving a strong adhesive bond. In fact, most wood adhesives require a lower MC for adequate adhesive penetration and curing reaction. A low $\mathrm{MC}$, which is close to the equilibrium moisture content (EMC), is desirable because this condition minimizes dimensional changes of a composite. Accordingly, defects such as warp, bow, twist, and crack are later negligible. Since a low MC is beneficial overall, drying of wood is not only a requirement but also an advantage.

Inactivation has been particularly problematic in the plywood industry, but it has also appeared in other wood-based composite processes, where wood is exposed to an excessive heat condition. Estimation has shown that the inactivation problem is associated with a loss of several million dollars yearly because these wood-based composites must be used for low-value purposes (Christiansen 1990), or they have to be withdrawn from the market. Besides economics, the greatest concern of wood inactivation is safety, because structures can fail and collapse as a consequence of a weak wood-adhesive bond. 


\subsection{Research Needs}

Many surface inactivation issues, caused by excessive heat exposure, are still undefined. A sufficient but simple method for determining wood surface inactivation has not been developed yet. Although the APA-The Engineered Wood Association developed a surface inactivation rating system, which is based on the comparative absorption time for a drop of water on sanded and unsanded veneer surfaces, the results are not directly applicable. Their evaluation does not assure that absorption of adhesive will behave in the same manner as water. Besides this, the APA method comprises only water absorption as a measure for predicting adhesion, but it should also include water wettability, which is often strongly related to adhesion. Therefore, a simultaneous measurement of absorption and wettability would provide more useful information on surface inactivation. Several other techniques have been proposed for quantifying wood surface inactivation (e.g., contact angle measurement, surface tension evaluation, spread wetting measurement, color intensity measurement, and chemical analysis by spectroscopy), but none of them have been universally accepted.

Mechanical testing is the most relevant indication of the adhesive bond performance because it gives information for designing a safe and efficient bonded structure. Therefore, observing the adhesion in situ either from a strength or an energy approach could serve as the most reliable method for detecting inactivation. However, in situ measurement of the adhesive bond performance has at least two shortcomings. First, a mechanical test can be employed only after the manufacturing process, thus an occurrence of the inactivated surface might be detected too late. Second, most of the mechanical tests are complex. They require expensive equipment, knowledge about the stress distribution, and often a special specimen preparation.

A remedy for inactivation is the next research need. In terms of adhesion, the remedy has to be simple, economical, and effective. The approach to the problem goes in two directions. Wood surface inactivation might be either inhibited before heat exposure or treated after its occurrence. Preventive measures comprise utilization of lower drying temperature or higher humidity levels within the dryer. Wood material can be classified according to $\mathrm{MC}$, and then dried at different drying schedules. A chemical treatment of the wood surface prior to drying is also a possibility. 
Once inactivation occurs, several measures might be used to increase wood wettability and to promote adhesion. Removing the surface inactivated layer by brushing, sanding, or planning is the most effective, but least desirable method. Surface removal requires an additional production step, it is not applicable for wood flakes and particles, and it wastes raw material. Thus, this measure is non-economical and often technically unfeasible.

Surface treatment with sodium hydroxide, calcium hydroxide, nitric acid, hydrogen peroxide, and borax usually improves adhesion, but the effect is rather small. These chemicals need to be applied to both surfaces. This is not common practice in the industry because application requires additional and special equipment. Thereafter, inclusion of wetting agents in the adhesive formulation-to promote both wettability and adhesion-would be a more operative method. A non-aqueous solvent can also be added to carry resin components into the wood cell wall where water cannot penetrate through the repellent hydrophobic layer. However, any modification, either of the surface or of the adhesive, presents an additional cost. Unfortunately, the most effective substances are usually the most expensive.

The listed measures provide just a partial improvement of adhesion, usually not sufficient for standard requirements. In addition, some of the proposed measures are not economical, while others involve only a partial solution or present a technical production problem. Thus, a satisfactory remedy for thermal inactivation for the wood-based composites industry is still unavailable.

\subsection{Hypotheses}

Inactivation alters physical and chemical properties of the wood surface. After excessive heat exposure, the wood surface exhibits decreased water wettability. Thus, it is assumed that the inactivation process modifies the primarily hydrophilic wood surface to a hydrophobic wood surface. This probably originates from either extractives migration to the surface, or from lignin concentration and rearrangement at the surface. Both, extractives and lignin, have hydrophobic character contrary to the other wood constituents (e.g., hemicelluloses), that have more hydrophilic character. Non-polar, hydrocarbon type of extractives should have the highest impact on severity of surface inactivation. 
Since the amount and character of extractives vary strongly with wood species, a difference in the severity of surface inactivation is expected. Removal of extractives by extraction should improve wettability and adhesion. The relationship between wettability and adhesion is postulated. This hypothesis originates from the necessity of sufficient wetting for an establishment of the attractive forces between wood and adhesive molecules. Thus, a chemical treatment of an inactivated wood surface, that promotes wettability or provides new bonding sites, should improve adhesive bond performance.

\subsection{Objectives}

This study deals with heat-induced wood surface inactivation. The purpose of this research project is, not only to collect new evidence and extend knowledge about the inactivation phenomenon, but also to provide remedies for inactivation in practical application. Within this scope, the objectives of the study are:

I. Identification of temperature and time exposure levels that cause wood surface inactivation for two wood species, including one hardwood and one softwood.

II. Chemical and physical characterization of wood surfaces in regard to inactivation by several different temperature exposures.

III. Identify the relationship among chemical composition of the wood surface, its wetting capacity, and its bonding performance.

IV. Reactivation of the inactivated wood surface by a chemical treatment to enhance wettability and to improve adhesion.

V. Development of a fast and sufficient method for detecting wood surface inactivation. 


\section{Chapter 2. Literature Review}

\subsection{Wood Surface Inactivation}

A freshly produced surface contains all of the molecular attractive forces that previously held the material together (Marra 1992). In a vacuum, most of the forces remain active for a long period, but some dissipate or become internally directed over time. In the atmosphere, a surface experiences changes such as gas adsorption, oxidation, and contamination with fine particles. Thus, the attractive forces become preempted and satisfied, leaving less to attract an adhesive (Marra 1992). Furthermore, reduction of surface forces can arise from inside of the material as diffusion and reorganization of molecules, and as migration of low molecular substances.

In the case of wood material, several mechanisms can reduce the attractive forces on the surface. Some proposed mechanisms are: migration of extractives to the surface, surface molecular reorientation, micropore closure, elimination of surface hydroxyl bonding sites, surface oxidation, chemical interference with resin cure (Christiansen 1991). These changes of a wood surface are reflected in surface inactivation. In adhesive bonding to wood, the Wood Handbook (USDA 1999) defines surface inactivation as "physical and chemical modifications of the wood surface that result in reduced ability of an adhesive to properly wet, flow, penetrate, and cure." Hancock (1963) introduced the term inactivation of a wood surface because this type of surface (i.e., inactive surface) had an adverse effect on the reactivity of phenolic adhesive. Troughton and Chow (1971) described surface inactivation as a heat-induced change in the wood veneer resulting in a loss of bonding ability. Generally, an inactivated wood surface does not bond well or causes poor adhesion. A low wettability associates with surface inactivation. Severe surface inactivation even prevents wetting to occur. When a wood surface is inactive, a broken adhesive bond often shows a bondline with the imprint of the opposite surface and an occasional loose fiber imbedded in the adhesive (Forbes 1998). The appearance of a wood grain pattern of the opposite adherend indicates that, even though adhesive penetrated into the voids of the wood surface and into the cell lumens, molecules of the adhesive did not wet the inactive surface and did not penetrate into wood cell wall. 


\subsection{Sources and Causes of Wood Inactivation}

Wood surface inactivation has many sources: air, light, wood, heat, chemical treatments, machines, and others (Marra 1992). Typical processes that lead to some degree of wood surface inactivation are wood aging, wood weathering, wood seasoning, and wood heating or drying. The later process is most significant and severe inactivation process in the wood-based composite industry, where wood surface usually experiences an excessive heat exposure.

A heat-induced inactivation comprises (1) primary inactivation, and (2) secondary inactivation. The primary wood surface inactivation refers to primarily wood exposure to heat as in first-time wood drying. That includes kiln drying, drum drying, jet drying, and hot-platen drying. The secondary wood surface inactivation refers to subsequent heat treatment, such as hot-pressing a composite to cure the adhesive. The scope of this study is wood surface inactivation that occurs when drying wood at elevated temperatures.

The exposure to a high temperature is the prevalent cause of wood surface inactivation. At the beginning of drying, a high MC provides a cooling effect on the surface when free liquid water is evaporated. As the MC of the wood falls below the fiber saturation point, the more strongly held bound water moves to the surface as a vapor, which does not produce the cooling effect that the evaporation of free liquid water does (Christiansen 1990). Thus, the wood surface temperature begins to increase, which raises the possibility for inactivation to develop. In the late stages of drying, when moisture content is very low, the surface temperature can exceed the safe limit and surface inactivation will occur.

A long exposure time, low relative humidity, and low wood moisture content aggravates inactivation (Christiansen 1990). Some inactivation-related phenomena, such as aging of the wood, can also occur at lower temperature over longer time. These phenomena include a reduction of hygroscopicity, wettability, and absorptivity. Hygroscopicity of wood represents an ability of wood to absorb or to desorb water in response to changes in the relative vapor pressure of the atmosphere (Haygreen and Bowyer 1996). Wettability refers to a condition of a surface that determines how fast a liquid will wet and spread or if it will be repelled and not spread on the surface (USDA 1999). Absorptivity of wood indicates an ability to assimilate gas or vapor by the surface (Haygreen and Bowyer 1996). 


\subsection{Factors Affecting Wood Surface Inactivation}

\subsubsection{Species Effect}

Some wood species are more susceptible to surface inactivation than others. Factors that have a prevalent influence on the inactivation process are wood anatomy, wood chemistry, and wood moisture content. A majority of the reported inactivation problems are related to softwood species (Christiansen 1990) because softwoods are usually more susceptible to inactivation than hardwoods. The temperature at which inactivation occurs is also dependant on wood species. Christiansen (1990) reported that the maximum safe drying temperature for avoiding inactivation in several softwoods varied with species. Southern pines were the most susceptible to inactivation, followed by ponderosa pine, inland Douglas-fir, western white pine, larch, and coastal Douglas-fir.

Heartwood overdries more easily than sapwood because it usually has a lower MC than sapwood (Troughton and Chow 1971). Thus, heartwood and sapwood possess different susceptibility to inactivation. Additionally, heartwood contains a higher proportion of the extractives than sapwood (Shupe et al. 2001). The extractives can affect wood wettability and adhesive spreading. Most of the extractives possess a hydrophobic character, thus they repel water. Kajita and Skaar (1992) attributed the greater wettability of sapwood compared with heartwood to the higher extractive content of the heartwood. Extractives' deposition can also block the pit openings between the wood cells. This reduces wood permeability and prevents penetration of the adhesive into the wood cellular structure.

Wood extractives tend to dominate the surface and thus, they significantly affect the surface properties. However, all the chemical components of the wood contribute to the surface chemistry (Gardner et al. 1995) and therefore, surface inactivation can originate from different wood constituents, not from extractives only. In fact, wood species with no or with a low amount of extractives can experience surface inactivation as well (Christiansen 1990). Extractive-free veneer could be inactivated, but the inactivation is accelerated when extractives are present (Wellons 1977). 


\subsubsection{Effect of High Temperature and Time}

Wood inactivation is a time-dependent process accelerated by increasing temperature. Surface inactivation can occur either at low temperature for a long time (i.e., aging), or in a short time at high temperature. However, high temperature causes more severe inactivation than aging. Also, mechanisms of inactivation change with temperature. The inactivation by pyrolysis begins at temperatures $>270^{\circ} \mathrm{C}$ (Fengel and Wegener 1989), while the inactivation mechanism of extractives migration occurs already at room temperature. For the most sensitive American coniferous species, significant wood surface inactivation occurs at the drying temperature of $160^{\circ} \mathrm{C}$ and higher (Christiansen 1990).

Even though wood surface inactivation can occur in many processes, most inactivation problems are associated with drying of veneer at high temperature. Veneer surface temperature changes during drying. At the beginning of this process, green veneer is warming up to a certain temperature, which mostly depends on wood specific gravity, wood $\mathrm{MC}$, and the drying temperature of the air. As wood dries, water moves toward the dry outer surfaces in the form of liquid water and water vapor (Siau 1995). At some point, steady-state conditions can be assumed. The total water vapor mass flux is constant. The water evaporation rate from the wood surface to the air is the same as the water flow rate from the bulk wood to the wood surface. In this case, veneer surface temperature is lower than air temperature because of evaporative cooling. As the MC decreases and falls below the FSP, wood contains only bound water. This water is held more strongly to wood by hydrogen bonding, thus the water diffusion from the bulk to the surface is slower than evaporation of water on the surface. The evaporative cooling effect decreases and the surface temperature starts to climb to temperatures near that of the air in the dryer (Christiansen 1990). This is the stage when typical wood surface inactivation occurs (Suchsland and Stevens 1968).

Many experiments have shown that high drying temperature reduces the wood adhesive bond strength, or that high temperature decreases wood hygroscopicity and hinders wettability. For instance, heartwood veneer of Douglas-fir dried at temperatures above $205^{\circ} \mathrm{C}$ suffered a loss in breaking strength when compared to veneer dried at $177^{\circ} \mathrm{C}$ or below (Christiansen 1990). 
The water absorption capacity of Douglas-fir heartwood veneer decreased with either increased dryer temperature or increased drying time. This relationship is often related to overdrying of wood. Overdrying inactivates the surfaces of Douglas-fir veneer, resulting in poor wettability (Kajita and Skaar 1992). The contact angle of a water droplet applied on thermally treated Fir increases with temperature (Figure 2.1), which indicates that surface wettability decreases with severity of thermal treatment (Podgorsk et al. 2000).

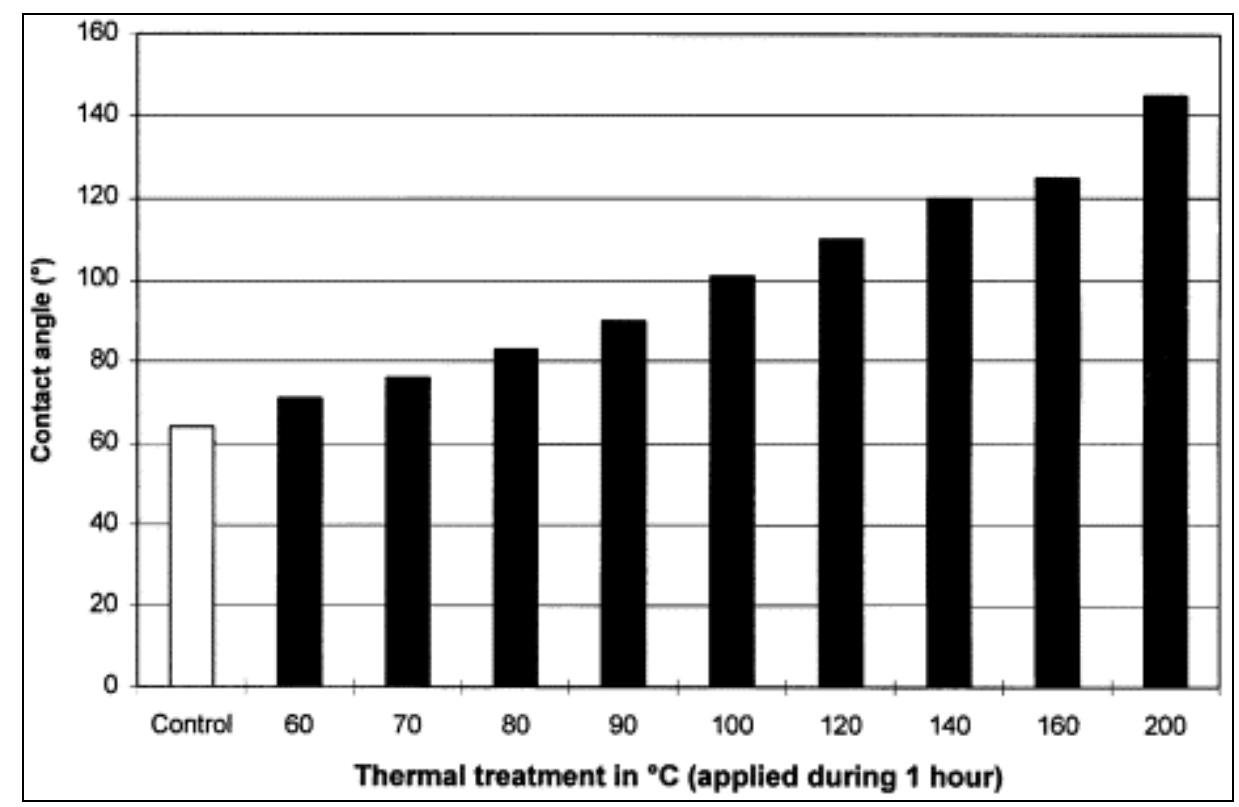

Figure 2.1. Influence of thermal treatment on Fir wettability (Podgorski et al. 2000).

Inactivation reduces veneer absorptivity, which could be a contributing factor in inferior joints because of reduction of wettability of veneer by the adhesive (Currier 1958). A low adsorbtion of water into the wood during the assembly period results in water retention within the bondline, which induces two effects. The water can excessively fluidize the resin, too much of which penetrates the wood, and the presence of excess water causes longer curing time (Northcott et al. 1959). 


\subsubsection{Effect of Drying Technique}

The drying technique affects inactivation because drying parameters (e.g., air temperature, velocity, and direction) vary with the technique. Shupe et al. (2001) found that oven drying, air-drying, and freeze-drying changed wood wettability in different ways. Contact angle was higher on oven-dried and air-dried wood surfaces than on freeze-dried wood surfaces. Therefore, low temperature drying diminishes or eliminates wood surface inactivation.

In a jet dryer, inactivation can occur at a rather low initial MC of veneer (Walters 1973). The increased surface drying rates increase the chance for surface inactivation before veneer is completely dried (Christiansen 1990). The air velocity and steam also play important roles in surface inactivation. For low temperatures and low air velocities, steam helps protect wood against charring better than air (Christiansen 1990). Kozlik (1974) found that the drying medium had no effect on wood failure, but drying with steam allowed slightly higher shear strength in southern pine plywood.

In a platen drying process, wood-veneer is placed between two hot plates. The contact between the plates and wood surface may influence wood surface properties during the drying. The aluminum plate provides an aluminum oxide surface and also excludes oxygen over much of the surface. A few researchers investigated the effect of this drying method on wettability and bondability of wood. Kadlec (1980) showed that the wettability of Douglas-fir veneer generally decreased with increased plate temperature. However, the trend was not strong or consistent. In spite of the studies that showed a possible inactivation effect caused by platen drying (Bohlen 1972; Koch 1964), no clear conclusion can be drawn.

\subsection{Mechanisms of Inactivation}

Several mechanisms can be involved in wood surface inactivation. Some are more prevalent than others, which depends on many factors. For instance, at low drying temperatures degradation by pyrolysis and oxidation are not significant, but these mechanisms become critical at very high temperatures. Usually, a wood surface inactivation is not due to a single phenomenon but rather a combination of a few. 
Christiansen $(1990,1991)$ reviewed the mechanisms that are most often proposed for wood surface inactivation. The inactivation mechanisms involving physical phenomena are: (1) extractives-related nonwetting, (2) surface molecular reorientation, and (3) micropore closure. A fourth possible mechanism, which is seldom a problem, presents contamination by soot or other airborne deposits (Christiansen 1990). The inactivation mechanisms involving chemical phenomena include: (1) elimination of surface hydroxyl bonding sites by ether formation (2) oxidation and/or pyrolysis of surface bonding sites, and (3) chemical interference with resin cure or bonding (Christiansen 1991). Some other inactivation mechanisms, especially with paper fibers, have been identified (e.g., hornification).

\subsection{Physical Mechanisms of Inactivation}

\subsubsection{Effect of Extractives on Wettability and Adhesion}

Time- and temperature-dependent changes of wood wettability have often been attributed to migration of extractives to the surface (Christiansen 1990). After thermal treatment of wood, the extractable compounds are responsible for poor wettability and weak adhesion (Podgorski et al. 2000). Gray (1962) evaluated advancing and receding contact angles for 19 wood species. Sanding the surfaces of specimens produced lower contact angles but the amount of the effect varied by species. Changes in contact angles were attributed to surface contamination by low molecular weight fatty acids, high extractives content, and high resin content.

Hse and Kuo (1988) reviewed the influence of extractives on wood gluing and finishing. According to their study, the extractives are common and important sources of surface contamination harmful to wood adhesion. Bonding strength is adversely affected by the degree of wood surface contamination. Deposition of extractives on the surface may reduce adhesive bond strength in many ways. High extractives concentration on the surface increases the possibility of contaminating and reducing the cohesive strength of the adhesive. Extractives may block reaction sites on wood surfaces and prevent adequate wetting by the adhesive. Oxidation of extractives tends to increase the acidity, which interferes with adhesive cure. 
The amount, the type, and the nature of extractives affect wood wettability. The quantity of extractives transported to the surface depends mainly on relative humidity and temperature. The relative humidity affects the moisture gradient, which promotes mass flow. Increased temperature improves extractives solubility and, it accelerates water movement. Water-soluble extractives are transported to the wood surface along with water during the drying operation and are deposited as solids when the water evaporates. Water-insoluble extractives may migrate to the wood surface in a vapor phase at high drying temperatures (Hse and Kuo 1988).

Wood extractives are polar and non-polar (Fengel and Wegener 1989). Non-polar extractives are primarily responsible for low wettability of a wood surface by water-borne adhesives. Nguyen and Johns (1979) found that wettability of Douglas-fir increased after extraction with benzene-alcohol because extraction removed low or non-polar components of the extractives from the surface. On the other hand, redwood showed a slight decrease of wettability after extraction. This happened because redwood contains other types of extractives than Douglas-fir and their removal probably did not affect wettability.

Troughton and Chow (1971) found that the amount of total fatty acids on white spruce veneer surfaces did not correlate with plywood bond quality. The results indicated that fatty acids play a minor role in the surface inactivation of white spruce veneer. Migration of wood resin to the surface of veneer was mentioned as a possible cause of poor wetting (Sellers 1977). Extractives were responsible for low wettability of southern pine bark (White et al. 1974). Hse and Kuo (1988) noted that extremely pitchy surfaces on southern pine veneer are not favorable for bonding. Pitch deposits, containing excess resin, can occur in conifers having resin canals: pines, Douglas-fir, spruces, and larches. For hardwood, substances such as natural latex, oleoresins, and phenolics present barriers to bonding (Christiansen 1990).

Removal of extractives by extraction with polar or non-polar solvents improves the wettability of many species. However, some studies did not find this relationship. Sometimes extractives are not removed completely, or some other inactivation mechanism may have a more significant effect on wettability. Maldas and Kamdem (1999) found that wettability of southern yellow pine decreased after extraction with ethanol-toluene. After the first extraction, the contact angle on the extracted surface was even higher (i.e., low wettability) than that obtained on the 
unextracted surface. The same result was observed after the second extraction with ethanol. Finally, the third extraction with water resulted in a contact angle similar to that of unextracted wood. Hancock (1963) found that extraction of veneer in a variety of different organic solvents prior to drying increased bondability, while post-drying extraction did not improve glue bond quality. However, a solution of sodium hydroxide or sodium carbonate, sprayed on dried wood, helped restore wood surface bondability to the certain degree, especially at longer assembly times (Christiansen 1990).

Even though extractives cause a decrease in wettability, and they can inhibit adequate bond formation, there is no clear conclusion about the effect of extractives on the susceptibility of a wood surface to inactivation. Some authors (Hancock 1963; Haskell et al. 1966; Koch 1972; Suchsland and Stevens 1968) found correlation between amount of extractives on a wood surface and degree of inactivation, but others did not (Troughton and Chow 1971).

\subsubsection{Molecular Reorientation at Surfaces}

Wood surfaces consist of three natural polymers: cellulose, hemicellulose and lignin. Polymer surfaces are time-, temperature-, and environment-dependent (Gunnells et al. 1994). Molecules of the polymer surface can reorient themselves to present a low energy surface against air. The driving force for reorientation is thermodynamics, with the surface tending to minimize its free energy. Amorphous and glassy polymers, such as hemicellulose and lignin in wood, are not in thermodynamic equilibrium (Gunnells et al. 1994). If molecular motions are possible, glassy polymers may rearrange to minimize surface free energy. This phenomenon was observed on hydrophilic hydrogels. Hydrophilic surfaces changed to hydrophobic ones upon exposure to air, but recovered upon exposure to an aqueous environment. This process is described as selfdiffusion of the polymer molecules (Gunnells et al. 1994).

Surface reorientation can be a part of the aging process in which surface wettability is reduced. Molecular reorientation results in fewer reactive groups remaining on the surface for chemical reaction or for secondary attraction to adhesive. Also, the surface is more hydrophobic after polymer reorientation. Hydrophobic surfaces have little or no tendency to adsorb water. Thus, hydrophobic surfaces of wood repel rather than attract water-borne adhesives. 
At high temperatures, reorientation and other molecular movements are accelerated, allowing faster formation of a hydrophobic surface. This is particularly pronounced when temperature and MC are such that hemicelluloses and lignin are above their glass transition temperatures. The glass transition of these two amorphous polymers strongly depends on moisture content. Hemicelluloses have a glass transition between -23 and $200{ }^{\circ} \mathrm{C}$ (Kelly et al. 1987), while lignin in softwoods and hardwoods has glass transition in the range of $65-85^{\circ} \mathrm{C}$ and $90-105^{\circ} \mathrm{C}$ respectively (Glasser 2000). Therefore, structural rearrangement of the amorphous part of the wood surface can likely occur when drying wood or curing wood-based composites. Compared to extractives migration, molecular rearrangements at the wood surface cause smaller changes in hydrophobicity than non-polar extractives.

\subsubsection{Micropore Closure}

One of the possible inactivation mechanisms of wood may relate to the micropore closure in the wood cell walls. Many micropores between the lamellae of the cell wall are lost during a first-ever drying process (Christiansen 1990). Increasing drying temperature loses more porosity. The sorption and diffusion properties of wood surfaces decrease after heat exposure. Micropore closure affects also adhesive penetration and wetting of the wood cell walls. The closure of larger micropores limits penetration by larger resin molecules and thus, the bond strength and wood failure decreases (Wellons 1980). This applies particularly in those cases where mechanical interlocking plays an important part of the adhesion.

\subsection{Chemical Mechanisms of Inactivation}

\subsubsection{Elimination of Surface Hydroxyl Bonding Sites}

The original hypothesis for the mechanism of inactivation was that water was eliminated from cellulose hydroxyl groups to form ether bonds. Ether bonds are less receptive to hydrogen bonding with polar adhesives than the original hydroxyl groups (Christiansen 1991). A loss of hygroscopicity is assigned to a gradual loss of wood hydroxyl groups during drying (Zavarin 1984). This mechanism cannot completely explain poor adhesion of thermally inactivated wood. 


\subsubsection{Oxidation and/or Pyrolysis of Surface Bonding Sites}

Oxidation and pyrolysis are real and inevitable inactivation mechanisms at high enough temperatures and long times. Increasing temperature accelerates this process and the time for degradation becomes shorter. The rate of degradation is much faster at extremely high drying temperature. At very high temperatures, the hemicelluloses may be changed to furfural polymers, which are less hygroscopic (Hillis 1984). Also, moisture content strongly catalyzes the depolymerization processes of wood constituents (Zavarin 1984). The oxidation process is relatively slow at the temperatures where inactivation is usually encountered in drying pines, Douglas-fir, and larch (Christiansen 1991). Conversion of wood components and significant occurrence of gaseous degradation products are observed at temperatures above $200^{\circ} \mathrm{C}$ (Fengel and Wegener 1989). Thus, oxidation is not a sufficient explanation for surface inactivation below $200^{\circ} \mathrm{C}$, even though combustion of wood components can start at temperatures around $167^{\circ} \mathrm{C}$ for lignin, and at $175^{\circ} \mathrm{C}$ for hemicelluloses (Christiansen 1991). However, oxidation and pyrolysis were proposed as a prime cause of surface inactivation for white spruce veneer (Troughton and Chow 1971). Hemingway (1969) concluded that the reduced wettability of yellow birchwood might be related to the oxidation of some fatty acids.

\subsubsection{Chemical Interference with Resin Cure or Bonding}

The alkaline or acidic nature of the wood surface could impede bonding by interfering with the cure of the resin. The curing of adhesives could be retarded, accelerated, or not affected by a changed $\mathrm{pH}$ value of the wood surface. The curing problem is more likely associated with species that have a high amount of acid extractives such as tropical hardwood species, pine, and oak. The acidity of oak surfaces significantly reduced the bond strength of resorcinolic adhesives (Subramanian 1984). Also, extractives often modify the cure of phenolic adhesives (Wellons 1977). The acidic extractives of oak and kapur prolonged the curing of phenolic adhesives (Hse and Kuo 1988). On the other hand, a low pH of extractives concentrated on the wood surface accelerates chemical the reactions of acid-catalyzed urea-formaldehyde adhesives. 


\subsection{Mechanism of Hornification}

A mechanism of hornification, which comprises a combination of physical and chemical phenomena, presents an alternative to previously mentioned mechanisms. Hornification is defined as the change in water sorption behavior that results from water removal, either at ambient or elevated temperature, and does not necessarily entail complete drying (Kato and Cameron 1999). In other words, hornification can be explained by irreversible intra-fiber hydrogen bonding during water loss. It has been observed in paper drying.

Typical temperatures used to promote hornification range between 80 and $120^{\circ} \mathrm{C}$, which is enough to promote drying without allowing thermal degradation. Higher temperature increases the rate of evaporation, and increases molecular mobility. Hornification causes lower fiber flexibility, lower water retention, increased brittleness, and more compacted pore structure of the cell wall (Kato and Cameron 1999). As a result of hornification, wood fibers exhibit poor wettability and/or adhesion. However, hornification starts to occur at significantly lower temperature than wood surface inactivation associated with bonding difficulties. Thus, the mechanism of hornification is an insufficient explanation for typical wood surface inactivation.

\subsection{Measures for Inhibiting Inactivation of Wood Surface}

Wood surface inactivation might be inhibited by two basic approaches: before drying or after drying. First of all, lower drying temperature could be used. This would be necessary in the final stage of drying when the wood surface temperature starts to approach that of the surrounding air. Second, over-drying of wood should be prevented. Moreover, raw material should be classified before drying according to MC and then, each group should be dried using different drying conditions. However, this requires frequent changes in the drying setup, which is often not practical in the industry. Third, higher humidity levels can be used within the dryer to avoid over-drying. A chemical treatment with different chemicals prior to drying would be the next possibility. Moreover, extraction of extractives from the wood surface prior to drying could improve the bonding, but again, this is not a reasonable procedure in the industrial production. 
Once wood surface inactivation occurs, several measures might be used to increase the wettability and adhesion. Brushing, sanding, and planning can remove the inactivated layer. Treatment with chemicals, such as sodium hydroxide, calcium hydroxide, nitric acid, hydrogen peroxide (Christiansen 1990), and borax (Chow 1975), can partially improve adhesion. Additionally, wetting or coupling agents can be included in the adhesive formulation to improve wettability. A non-aqueous solvent can be added to the adhesive mixture to carry resin components into the wood where water cannot penetrate (Sellers 1985). Moreover, more aggressive adhesives can be used instead of conventional wood adhesives. An adhesive with low molecular weight, low viscosity, and low surface tension can better penetrate and wet inactive wood surfaces.

\subsection{Possible Remedies for Surface Inactivation}

Several investigations have been conducted to improve adhesion of inactivated wood surfaces, but no comprehensive and satisfactory solution has been found so far. Gardner and Elder (1990) found that chemical surface treatments (Gardner et al. 1991b) improved modulus of rupture (MOR) and modulus of elasticity (MOE) of the flakeboards, but the same treatment diminished internal bond and dimensional properties of the boards. Chow (1975) concluded that an aqueous borax solution reduced surface inactivation of freshly peeled Douglas-fir, white spruce, and lodgepole pine veneers. A similar effect was achieved with boric acid, but this caused corrosion problems.

Tris (polyoxyethylene) sorbitan monooleate improved the bond strength of Douglas-fir dried at $177^{\circ} \mathrm{C}$ or higher (Christiansen 1990). This chemical has to be applied prior to drying since treatment of wood surfaces after drying was not effective. A high cost of the chemical might limit its use in the industrial production. Christiansen (1990) also reported a beneficial bonding effect from the application of a $10 \%$ sodium hydroxide solution to the wood surface. The treatment was deleterious to white oak, but did help several other species bonded with casein resin. A similar effect was achieved with a calcium hydroxide solution. 
Techniques such as plasma treatment, and corona treatment (Podgorski et al. 2000), or flame ionization (Winfield et al. 2001) can improve wettability and adhesion of wood surfaces. However, Winfield et al. (2001) found that wettability improvement by oxidative activation with flame treatment depends on wood species. Oak and Meranti surfaces, both hardwood species, exhibited better wettability (i.e., a low contact angle) after flame treatment, while softwood species did not. Additionally, the surface energy increased after flame treatment for all three species, but it remained constant for hardwoods. Oxidative treatment or other plasma treatments often lead to better surface wettability (Figure 2.2) and to improved adhesion. However, application of these methods to wood-based composite material is usually limited by wood geometry (e.g., wavy veneer or small flakes), or hindered by process requirements (e.g., vacuum and speed).

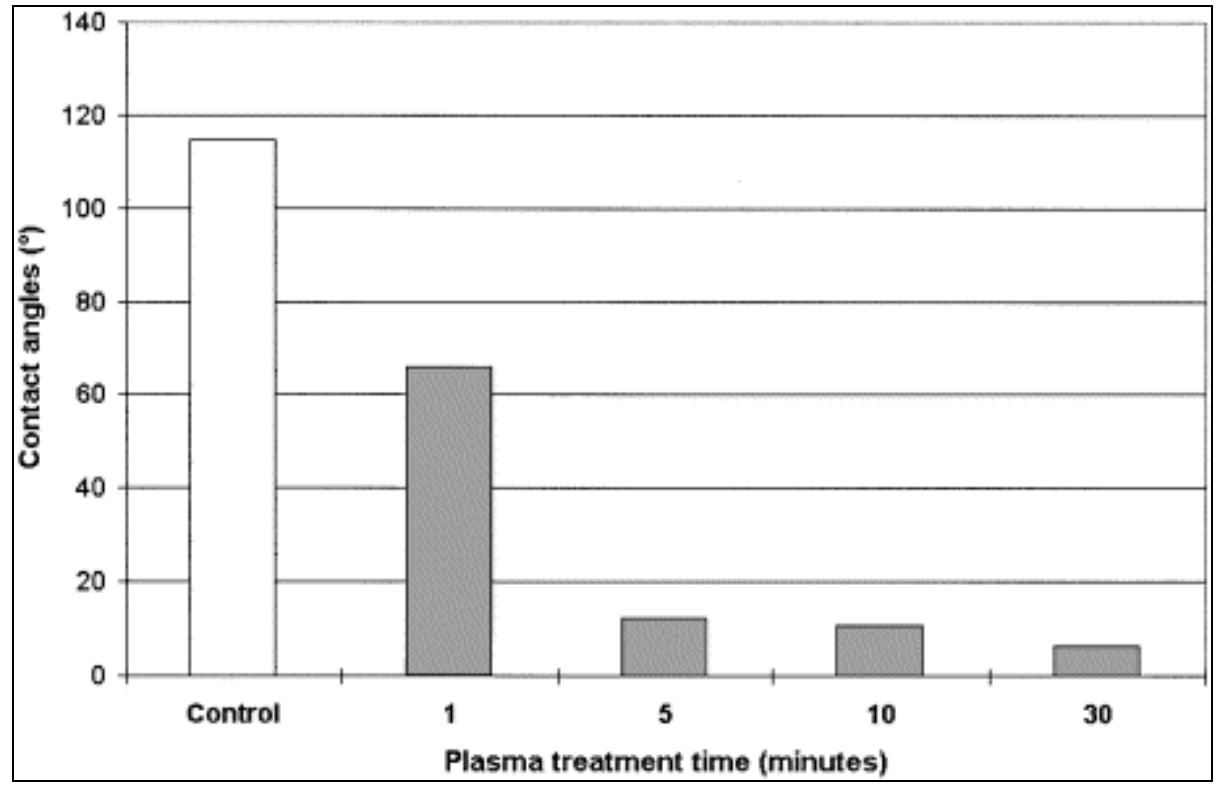

Figure 2.2. Influence of the plasma treatment time on fir wettability (Podgorski et al. 2000). 


\subsection{Surface Characterization}

Since wood inactivation is a surface phenomenon, understanding surface characteristics is of utmost importance for battling with inactivation problems. Surfaces rarely reveal the same properties as the bulk material. Surface properties are usually modified by several other causes, such as contamination, gas adsorption, oxidation, and surface rearrangements. Surface molecules are also surrounded in a different manner than bulk molecules. Atoms or molecules at the surface have some unconnected bonds and/or they cannot completely interact with surrounding molecules or atoms (Tsujii 1998). Thus, surface molecules have excess free energy. This difference between the energy of molecules located at the surface and in the bulk phase of a material manifests as surface free energy or as surface tension (Evans and Wennerström 1999).

Considering a lignocellulosic material, the chemical composition of a wood surface does not necessarily correspond to the chemical composition of the bulk of the wood. A wood surface is commonly richer in lignin and extractives than the bulk of the wood (Zavarin 1984). This can be a consequence of manufacturing processes, which may affect physical and chemical properties of the surface. For instance, the surface of wood pulp fiber contains up to ten times more lignin than the bulk of the fiber (Li and Reeve 2000) because the fiber surface was enriched with lignin during kraft pulping (Figure 2.3).

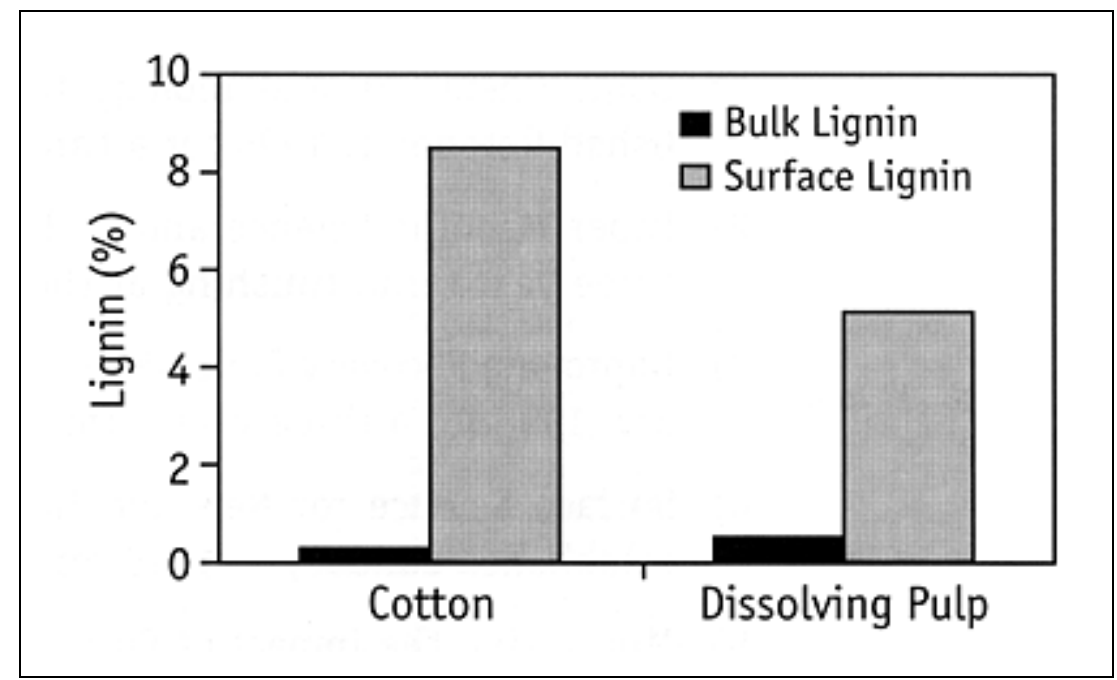

Figure 2.3. Lignin deposition on fiber surfaces after kraft pulping (Li and Reeve 2000). 
Surface characteristics are also modified when a wood surface is exposed to air and humidity. This exposure usually lowers surface free energy, which is undesirable in terms of wood wettability and wood adhesion. The most severe reduction of the surface free energy of wood occurs during thermal inactivation. In order to evaluate and quantify the severity of thermal inactivation, the measurements of surface chemistry, surface wettability, and adhesion between bonded surfaces need to be performed.

\subsection{Chemical Characterization of Surface}

Surface analytical methods differ from methods for bulk analysis because the object of observation is quite different. Figure 2.4 represents the regimes of surface analysis, thin film analysis and bulk analysis. In a general sense, the surface of the solid is defined as the outermost atomic layer, including foreign atoms absorbed into it and those adsorbed to it (Hagstrum 1972). In a chemical sense, "surface" refers to a phase boundary (Hiemenz and Rajagopalan 1997). In a strictly geometrical sense, "surface" has area but not thickness.

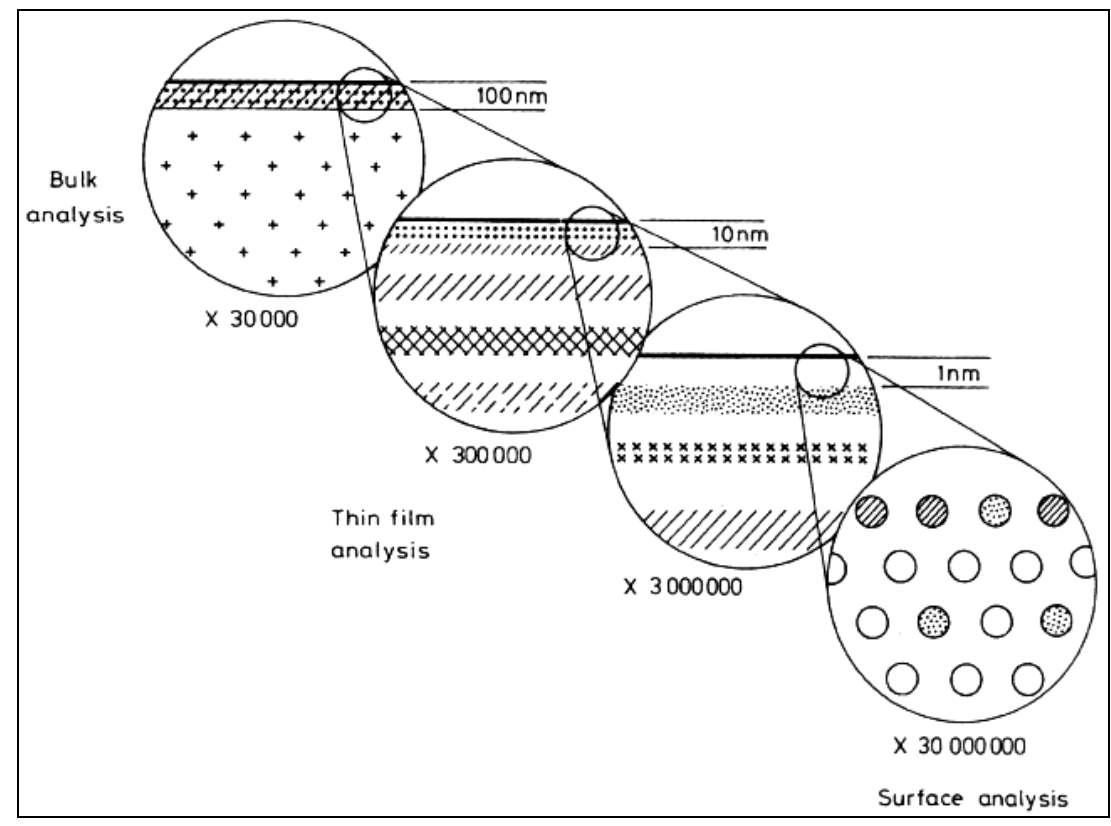

Figure 2.4. The regimes of surface analysis, thin film analysis and bulk analysis (Briggs and Seah 1990). 
Special equipment is needed to analyze surface properties of a material. Many analytical techniques have been developed for surface characterization. The names and acronyms of several popular surface analysis techniques are listed in Table 2.1. Among of the most popular techniques (Table 2.2), X-ray photoelectron spectroscopy (XPS) has the most benefits. The success of this technique is contributed to Kai M. Siegbahn, who shared the 1981 Nobel Prize in Physics for the development of high-resolution XPS (Hollander et al. 1981).

Table 2.1. Common acronyms for surface analysis techniques (Brune et al. 1997).

\begin{tabular}{|c|c|c|}
\hline Main acronym & Other acronym & Name of technique \\
\hline AES & SAM & Auger Electron Spectroscopy \\
\hline APFIM & FIM-AP, FIM & Atom Probe Field Ion Microscopy \\
\hline HREELS & & High Resolution Electron Energy Loss Spectroscopy \\
\hline ISS & LEIS & Ion Scattering Spectroscopy \\
\hline MEIS & & Medium-Energy Ion Scattering \\
\hline RBS & BS & Rutherford Backscattering Spectrometry \\
\hline SIMS & & Secondary Ion Mass Spectrometry \\
\hline UPS & & Ultraviolet Photoelectron Spectroscopy \\
\hline XPS & ESCA & X-ray Photoelectron Spectroscopy \\
\hline
\end{tabular}

Table 2.2. Survey of the popular techniques for surface analysis (Briggs and Seah 1990)

\begin{tabular}{|c|c|c|c|c|c|c|c|c|c|c|c|}
\hline Technique & $\begin{array}{c}\text { Information } \\
(\mathbf{E}= \\
\text { elemental, } \\
\mathbf{C}= \\
\text { chemical })\end{array}$ & $\begin{array}{l}\text { Spatial } \\
\text { resol- } \\
\text { ution } \\
\text { (best) }\end{array}$ & $\begin{array}{c}\text { Sampling } \\
\text { depth } \\
\text { mono- } \\
\text { layers }\end{array}$ & $\begin{array}{c}\text { Sensi- } \\
\text { tivity } \\
\text { (order } \\
\text { of) }\end{array}$ & $\begin{array}{l}\text { Quanti- } \\
\text { fication } \\
(\sqrt{ }=\text { easy })\end{array}$ & $\begin{array}{l}\text { Elements } \\
\text { not } \\
\text { covered }\end{array}$ & $\begin{array}{l}\text { Popu- } \\
\text { larity }\end{array}$ & $\begin{array}{c}\text { Specimen } \\
\text { prepara- } \\
\text { tion } \\
(\sqrt{ }=\text { easy) }\end{array}$ & $\begin{array}{c}\text { Ease } \\
\text { of } \\
\text { use }\end{array}$ & $\begin{array}{l}\text { Extent } \\
\text { of } \\
\text { support } \\
\text { data }\end{array}$ & $\begin{array}{c}\text { Effective } \\
\text { take-off } \\
\text { year }\end{array}$ \\
\hline $\begin{array}{l}\text { AES } \\
\text { Atom }\end{array}$ & $E^{\dagger}$ & $5 \mathrm{~nm}$ & 3 & $0.3 \%$ & $\sqrt{ }$ & $\mathrm{H}, \mathrm{He}$ & $* * * *$ & $\sqrt{ }$ & $* * * *$ & $* * * * *$ & 1968 \\
\hline probe & & & & & & & & & & & \\
\hline FIM & $\mathbf{E}^{\ddagger}$ & $1 \mathrm{~nm}$ & 1 & $1 \%$ & $\sqrt{ }$ & & $*$ & & $*$ & $* *$ & 1968 \\
\hline HREELS & C & $1 \mathrm{~mm}$ & 1 & $1 \%$ & & & $*$ & $\sqrt{ }$ & $* *$ & $* *$ & 1970 \\
\hline ISS & $\mathbf{E}$ & $1 \mathrm{~mm}$ & 1 & $1 \%$ & $\sqrt{ }$ & $\mathrm{H}, \mathrm{He}$ & * & $\sqrt{ }$ & $* * *$ & $* * *$ & 1967 \\
\hline MEIS & $\mathbf{E}$ & $1 \mathrm{~mm}$ & 3 & $1 \%$ & $\sqrt{ }$ & $\mathrm{H}, \mathrm{He}, \mathrm{Li}, \mathrm{Be}$ & * & & $* * *$ & $* * *$ & 1967 \\
\hline $\begin{array}{l}\text { RBS } \\
\text { SIMS }\end{array}$ & $\mathbf{E}$ & $1 \mathrm{~mm}$ & 100 & $1 \%$ & $v_{1}$ & $\mathrm{H}, \mathrm{He}$ & ** & & $* * * *$ & $* * * *$ & 1967 \\
\hline (static) & $\mathrm{C}$ & $1 \mu \mathrm{m}$ & 2 & $0.01 \%$ & & & ** & $\sqrt{ }$ & $* *$ & *** & 1970 \\
\hline $\begin{array}{l}\text { SIMS } \\
\text { (dynamic, }\end{array}$ & & & & & & & & & & & \\
\hline imaging) & $\mathbf{E}$ & $20 \mathrm{~nm}$ & 10 & <1 p.p.m. & $\sqrt{ }^{8}$ & & $* * *$ & $\sqrt{ }$ & $* * *$ & $* * * *$ & 1968 \\
\hline $\begin{array}{l}\text { SIMS } \\
\text { (dynamic, } \\
\text { depth }\end{array}$ & . & & & & & & & & & & \\
\hline $\begin{array}{c}\text { prof.) } \\
\text { SNMS }\end{array}$ & E & $50 \mu \mathrm{m}$ & 10 & $<1$ p.p.m. & $\sqrt{ }^{5}$ & & $* * *$ & $\sqrt{ }$ & $* * *$ & $* * *$ & 1975 \\
\hline $\begin{array}{l}\text { (dynamic, } \\
\text { depth }\end{array}$ & & & & & & & & & & & \\
\hline prof.) & $\mathbf{E}$ & $50 \mu \mathrm{m}$ & 10 & $<1$ p.p.m. & $\sqrt{ }$ & & ** & $\sqrt{ }$ & $* * *$ & ** & 1970 \\
\hline UPS & C & $5 \mu \mathrm{m}$ & 3 & $1 \%$ & & N/A & $* *$ & $\sqrt{ }$ & $* * *$ & $* * * *$ & 1969 \\
\hline XPS & C,E & $5 \mu \mathrm{m}$ & 3 & $0.3 \%$ & $\sqrt{ }$ & $\mathrm{H}, \mathrm{He}$ & $* * * *$ & $\sqrt{ }$ & $* * * *$ & $* * * * *$ & 1967 \\
\hline
\end{tabular}




\subsubsection{X-Ray Photoelectron Spectroscopy}

X-ray photoelectron spectroscopy, also referred to as electron spectroscopy for chemical analysis (ESCA), is a very powerful non-destructive surface analytical technique (Reeve and Tan 1998). This technique provides valuable data on chemical surface composition and surface reorganization (Schrader and Loeb 1992). XPS also provides information on the oxidation state or chemical bonding state of elements.

Photoelectron spectroscopy probes only the surface region of solids. As a result, the technique is frequently used in investigations of phenomena such as absorption, corrosion, and adhesion, where surface chemical composition is of great importance (Ho 1982). XPS is widely used for surface analysis of polymers. This technique can also be used for surface analysis of wood to characterize its chemical composition, and also, to identify the concentration of wood components (i.e., polysaccharides, lignin, and extractives) on the surface.

The principle of the XPS/ESCA technique is the emission of electrons from atoms by absorption of photons (Brune et al. 1997). Electrons are held in the atom by a binding energy, which depends on the atomic charge distribution. The binding energy $\left(E_{B}\right)$ of an electron can be determined by measurement of the kinetic energy $\left(\mathrm{E}_{\mathrm{kin}}\right)$ of the photoelectron:

$$
\mathrm{E}_{\mathrm{B}}=\mathrm{h} v-\mathrm{E}_{\mathrm{kin}}-\phi
$$

Equation 2.1

where $h v$ is the energy of the characteristic X-ray, h is Planck's constant $\left(41.6 \times 10^{-16} \mathrm{eVs}\right), v$ is the frequency, and $\phi$ is the work function of the spectrometer (Brune et al. 1997). The information from XPS is inherently quantitative (Beamson and Briggs 1992). The binding energy is a characteristic of the atoms, which can be used for elemental identification (Reeve and Tan 1998). For example, carbon bound to itself and/or hydrogen only, has binding energy of $285.0 \mathrm{eV}$ and oxygen O1s has binding energy of around 533eV (Briggs and Seah 1990). All elements except hydrogen can be detected (Birdi 1997). If an element is involved in a chemical bond, then its binding energy will change (Young et al. 1982). This results in a chemical shift, which can be measured and used for the determination of the individual chemical states of atoms. For example, oxygen induces shifts to higher binding energy by $1.5 \mathrm{eV}$ per $\mathrm{C}-\mathrm{O}$ bond. The determination of the chemical states of atoms is the main advantage of the XPS/ESCA method. 
The XPS instrument includes the X-ray source, the monochromator, the sample stage, the lens, the analyzer, the detector, and a computer. The sample preparation and mounting are not critical; however, it is important to ensure a clear and uncontaminated surface. The high vacuum requirement $\left(10^{-8}\right.$ torr $)$ restricts the use of this technique for in situ measurements in nonvacuum environments. During the vacuuming, a sample is cooled down to minimize the influence of air molecular motions on the spectra. X-rays are applied after that. They are irradiated from $\mathrm{Mg} \mathrm{K} \alpha$ (1253.6 eV) or from Al K $\alpha(1486.6 \mathrm{eV})$ (Briggs and Seah 1990).

XPS analyzes a small area of a few square millimeters. A surface depth of about 10-50 is usually observed. The electrons of the atoms, which are deeper, are not able to escape and are not detected. The sampling depth depends on the escape depth and the incident angle of the Xray (Figure 2.5).

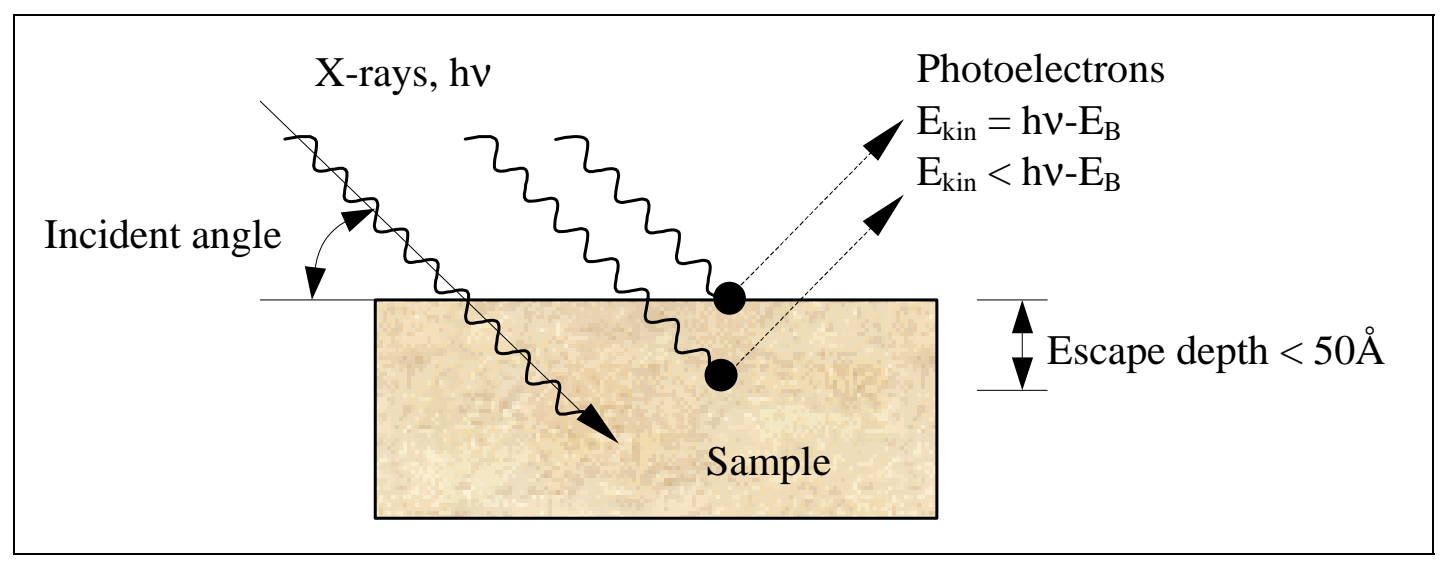

Figure 2.5. Escape characteristic of photoelectrons in XPS.

The interpretation of measurements is based on the standardized database for atoms and their shifts. For most studies, it is important to determine the relative concentration of the various constituents of the surface. The ratio of the elements is calculated based on the atomic sensitivity factor and on the curve area under each peak for the detected element. Detailed analysis comprises theoretical and experimental knowledge on the chemistry of the observed surface. 


\subsection{Wettability and Contact Angle}

Wettability is defined as a condition of a surface that determines how fast a liquid will wet and spread on the surface or if it will be repelled and not spread on the surface (USDA 1999). When contact angle is zero, perfect wetting of a surface occurs. The liquid spreads spontaneously or completely on the surface of the solids (Baier et al. 1968). Contact angle is an angle formed between the surface of a solid and the line tangent to the droplet radius from the point of contact with the solid (Figure 2.6).

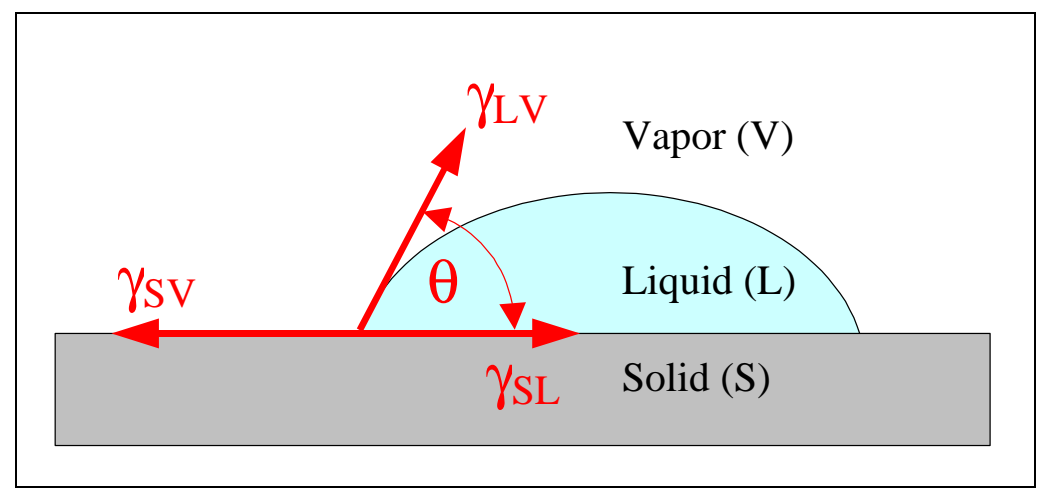

Figure 2.6. Contact angle and interfacial surface tensions at equilibrium.

Since the tendency for the liquid to spread increases as contact angle decreases, the determination of contact angles is a useful inverse measure of spreadability or wettability (Zisman 1964). In fact, the cosine of contact angle (i.e., the index of wettability) is often used as a direct measure of wettability (Kajita and Skaar 1992). When in mechanical equilibrium, the relationship among surface free energies and the contact angle $(\theta)$ for a liquid drop on a solid surface is expressed by Young's equation (Zisman 1964):

$$
\gamma_{S V}-\gamma_{S L}=\gamma_{L V} \cos \theta
$$

Equation 2.2

where $\gamma$ is interfacial surface tension, $\mathrm{S}$ is solid, $\mathrm{L}$ is liquid, and $\mathrm{V}$ is vapor (Fig. 2.5). The relationship among surface tensions can be extended to Dupre's equation:

$$
W_{S L}=\gamma_{S V}+\gamma_{L V}-\gamma_{S L}
$$


The work of adhesion, $\mathrm{W}_{\mathrm{LS}}$, represents the amount of work, which must be expanded to separate a unit of solid surface from liquid. The combination of equations (2.2) and (2.3) yields to the original Young-Dupre equation, which has been one of the most useful tools in the experimental approach to studying surface behavior (Collett 1972):

$$
W_{S L}=\gamma_{L V}(1+\cos \theta)
$$

\section{Equation 2.4}

The previous three equations neglect the factor $\pi_{\mathrm{SV}}$, which represents the change in the surface free energy upon adsorption of the vapor of the contacting liquid (Collett 1972). This value can be determined from the differential heat of sorption. However, in some cases this is not applicable, and also, this value is often negligibly small.

Collett (1972) concluded that the bulk of the evidence in the literature points to the fact that the measurement of the contact angle is the best experimental approach to assessing the phenomena of wetting. An intimate contact on a molecular level is assumed to be necessary for bond formation to achieve good adhesion between materials. This is thought to occur through the phenomena of wetting and spreading (Schmidt 1998). The spreading coefficient (S) is given by (Bateup 1981):

$$
\begin{array}{ll}
S=\gamma_{S V}-\left(\gamma_{L V}+\gamma_{S L}\right) & \text { Equation } 2.5
\end{array}
$$

A liquid will spread spontaneously on a solid surface when the spreading coefficient is greater than, or equal to, zero $(S \geq 0)$. This is achieved when:

$$
\gamma_{L V} \leq \gamma_{S V}-\gamma_{S L}
$$

\section{Equation 2.6}

Therefore, the changes in any of the three interfacial surface tension values can lead to a change of the spreading coefficient.

Contact angle and surface tension of a liquid can easily be determined in many ways. Often used techniques are the sessile drop method and the Wilhelmy plate method. The sessile drop method (Figure 2.1) is a simple method, which provides a direct value for the contact angle by laying a tangent on the outside of the drop (Adamson 1990). The Wilhelmy plate technique measures the force needed to balance forces originating from surface tension (Birdi 1997). 
On the contrary, the determination of surface energy of solids is indirect and complicated. One of the first approaches to the characterization of low-energy solid surface was an empirical one developed by Zisman and co-workers (1964). They established that a linear relationship often existed between the cosine of the contact angle of several liquids and their surface tension (Figure 2.7). Zisman introduced the concept of critical surface tension, which represents a value of the surface energy of an actual or hypothetical liquid that will just spread on the solid surface, giving a zero contact angle (Schrader and Loeb 1992). The meaning of "critical surface tension" is not the surface tension of the solid but only an empirical parameter closely related to this quantity. However, Zisman (1964) stated that critical surface tension is an even more useful parameter because it is a characteristic of the solid only.

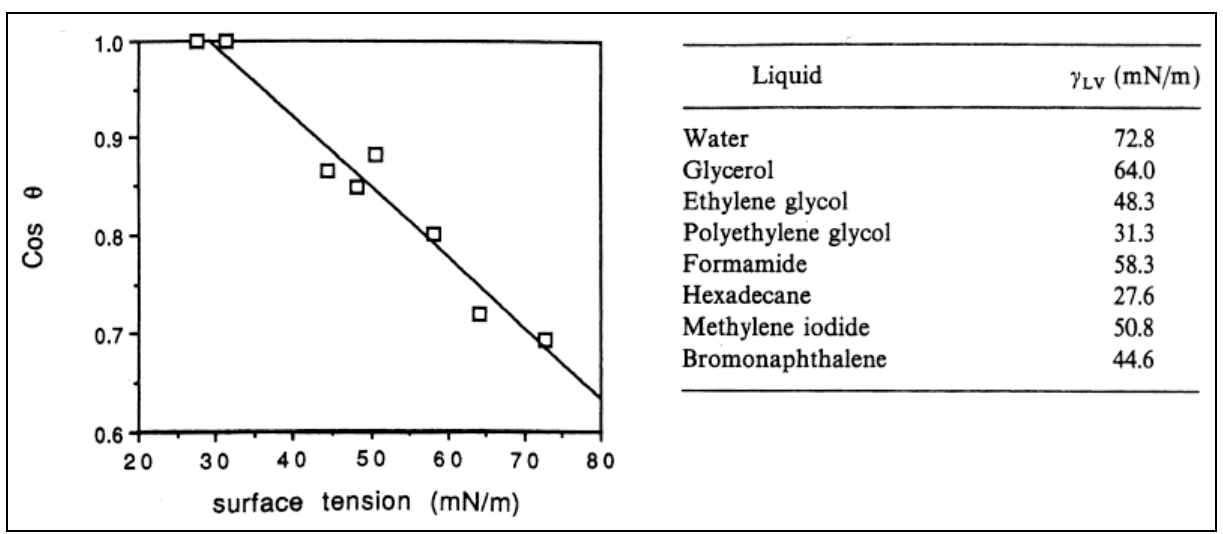

Figure 2.7. Critical surface tension plot (Schrader and Loeb 1992).

Evaluation of surface free energy of wood by the Zisman approach is feasible but limited. First, chemical heterogeneity, surface roughness, and hygroscopicity of wood impede precise measurements of contact angle (Gardner et al. 1991a). Since porous and hygroscopic wood absorbs water into its structure, the contact angle changes over time. Second, the contact angle also depends on the wood species, extractives present in wood, wood anatomy, wood surface sections, wood seasoning, moisture content, temperature, and surface roughness (Maldas and Kamdem 1999). Moreover, swelling of the wood surface (Wellons 1977), and contamination of the probe liquid with soluble wood extractives (Wålinder and Johansson 2001), also affect contact angle measurement. Therefore, the equilibrium condition cannot be achieved. 
Thus, the validity of the thermodynamic wettability principles for a wood surface is limited. But the results from contact angle measurements can be used as a relative measure when comparing among several wood surfaces. Also, the time dependent behavior of a drop of water on the wood surface provides a good early indicator of how the water-borne adhesive might later behave. A high surface free energy of wood and a low surface energy of the adhesive are desirable. These conditions promote wetting and spreading of the adhesive. There is evidence about the positive relationship between wood wettability and adhesion (Bodig 1962; Collet 1972; Wellons 1977). Since wettability often correlates with adhesion, the adhesive bond quality of inactivated wood can be partially predicted based on wettability measurements. Hse (1972) reported that contact angle is a useful index of adhesive effectiveness. However, mechanical testing is the most relevant indication of adhesive bond performance because it gives information for designing safe and efficient bonded structures.

\subsection{Adhesion and Adhesive Bond Performance}

Adhesion-a term referring to the attraction between the substances (Kinloch 1987) is a surface phenomenon (Wegman 1989). The nature and condition of the adherend surface are critical to the success of any bonding (Gauthier 1995). For instance, a rough surface provides more surface area than a smooth one of the same gross dimension. Surface chemical composition can differ from that of the bulk, and the surface may be contaminated by impurities. In order to evaluate the effect of wood surface properties on adhesion performance, an adequate testing method has to be employed.

Many tests have been developed for testing wood adhesive bonds: compression shear block, tensile shear for laminates, internal bond test for flake/fiber composites, and lap-shear for adhesives (Schmidt 1998). Most of these tests create stress states that promote wood fracture, thus adhesion is not adequately measured because wood failure dominates. There are several limitations, which hinder the capability of these tests in accurately evaluating the adhesive performance (Schmidt 1998). For instance, the most significant factors affecting the results of a shear tests are grain angle, grain orientation, specific gravity, proportion between earlywood and latewood, and stress concentration. 
An alternative to the above listed tests is a fracture mechanics approach. Fracture mechanics studies the formation of new cracks or the enlargement of existing ones as a result of an applied load. The process of crack development is described in four phases (Bodig and Jayne 1982): nucleation, initiation, propagation, and arrest. At low stress levels, the average size of cracks increases, but the material remains in a state of reversible equilibrium. As stress increases, larger cracks form, and when a critical size is reached, initiation begins. Continuous action of the external stress extends the crack even further, which is propagation. If the crack extends into a region capable of resisting the stress at the tip, propagation is terminated. Additional extension occurs only if the load is increased further, in which case a new initiation condition is reached. In the case of low resistance, the crack expands and catastrophic failure results (Bodig and Jayne 1982).

There are three basic modes of transferring loads between members of an adhesivebonded assembly: Mode I-opening or cleavage mode, Mode II-in plane shear, and Mode IIItearing or transverse shearing mode (Ebewele et al. 1979). The opening mode is the most suitable fracture test because the specimen (grain direction) can be oriented in a way, which keeps crack propagation within the bondline (Frazier et al. 2000). This prevents wood failure so that test data reveal more information about adhesion itself (Gagliano and Frazier 2001).

The double cantilever beam (DCB) is one of the most popular test specimen geometries used to measure adhesive fracture energy (Blackman et al. 1991). The most important parameter, determined from fracture testing, is the critical strain energy release rate $\left(\mathrm{G}_{\mathrm{C}}\right)$. This is a measure of the energy required to create two new surfaces through fracture of the adhesive bond.

Gagliano and Frazier (2001) introduced two significant improvements in the fracture cleavage testing of adhesively-bonded wood: (1) the flat DCB geometry and (2) data analysis using a shear corrected compliance method. The flat double cantilever beam geometry greatly simplifies sample preparation. The shear corrected compliance method accounts for variations in wood modulus, and it corrects a crack length measurement due to shear effect in wood adherends (Gagliano and Frazier 2001). 


\subsubsection{Shear Corrected Compliance Method}

Assuming that the specimen behaves in a linear-elastic manner upon loading, the mode I fracture energy $\left(\mathrm{G}_{\mathrm{I}}\right)$ is given by (Blackman et al. 1991):

$$
G_{I}=\frac{P^{2}}{2 B} \frac{d C}{d a}
$$

Equation 2.7

where $\mathrm{P}$ is the load, $\mathrm{B}$ is the width of the specimen, and $\mathrm{dC} / \mathrm{da}$ is the change in compliance, $\mathrm{C}$, with the change in crack length, a. From the simple beam theory approach, compliance is given by:

$$
C=\frac{\Delta}{P}=\frac{3 a^{3}}{2 E_{s} I}
$$

\section{Equation 2.8}

where $\Delta$ is displacement, $\mathrm{E}_{\mathrm{s}}$ is modulus of elasticity of the adherend or substrate, and I is second moment of area. Now, $\mathrm{G}_{\mathrm{I}}$ can be represented by:

$$
G_{I}=\frac{P^{2} a^{2}}{B E_{s} I}
$$

\section{Equation 2.9}

This approach to the calculation of $\mathrm{G}_{\mathrm{I}}$ is often referred to as the direct compliance method. If the adherends possess a low ratio of plane shear to axial modulus (e.g., wood), flexure of adherends causes shear forces to develop at small crack lengths. In order to correct the effect of shear, a shear corrected compliance method developed by Hashemi et al. (1990) is used (Gagliano and Frazier 2001):

$$
G_{I}=\frac{P_{c}^{2}(a+x)^{2}}{B(E I)_{e f f}}
$$

Equation 2.10

where $\mathrm{x}$ is shear correction factor or the crack length offset, and (EI) $)_{\text {eff }}$ is the effective flexural rigidity of the DCB specimen. These two parameters may be found experimentally by the following relationship (Gagliano and Frazier 2001):

$$
(E I)_{e f f}=\frac{2}{3 m^{3}}
$$




$$
x=\frac{b}{m}
$$

\section{Equation 2.12}

where $\mathrm{m}$ and $\mathrm{b}$ are the slope and the $\mathrm{y}$-intercept, respectively, from the linear trendline of the plot of the cube root of compliance versus measured crack length. The cubic relationship between compliance and crack length is derived from the beam theory.

According to ASTM D 3433-93 (1997), the DCB test method may be conducted to measure the fracture energy of a bonded joint, which is influenced by the adherend's surface condition, adhesive-adherend interactions, and primers. Since wood inactivation is a surface phenomenon, DBC should be very adequate test geometry for evaluating the influence of wood surface inactivation on adhesive bond performance. 


\section{Chapter 3. Characterization of Thermally Inactivated Wood Surfaces}

\subsection{Introduction and Problem Definition}

Wood surface inactivation, which results in a poor bonding ability with an adhesive, is a time-dependent process accelerated by increasing temperature. Surface inactivation can occur either at low temperature by long time (i.e., aging), or in short time at high temperature. However, high temperature causes more severe inactivation than aging. Also, the mechanisms of inactivation change with temperature. Several physical and chemical inactivation mechanisms can reduce the attractive forces on the wood surface, which are initially available for bonding with adhesive (Christiansen 1991). Each of the inactivation mechanisms can operate in different situations as well as functioning simultaneously (Carpenter 1999).

The severity of the surface inactivation depends on wood moisture content (MC), temperature level, and duration of temperature exposure. During a drying process, a significant reduction in bonding ability occurs at the end of drying, when the evaporative cooling effect decreases and the wood surface temperature approaches that of the air in the dryer (Suchsland and Stevens 1968). The temperature of a wood surface changes substantially during drying. At the beginning of drying, green wood warms up to a certain temperature, which mostly depends on wood specific gravity, wood MC, and the drying temperature of the air. As wood dries, water moves toward the dry outer surfaces in the form of liquid water and water vapor (Siau 1995). The water evaporation rate from the wood surface to the air is similar to the water flow rate from the bulk wood to the wood surface. In this case, the wood surface temperature is lower than the air temperature because of evaporative cooling. As MC decreases and falls below the fiber saturation point (FSP), wood contains only bound water. Wood holds this water more strongly (i.e., hydrogen bond), thus the water diffusion from the bulk to the surface is slower than the evaporation of water on the surface. The evaporative cooling effect decreases and the surface temperature starts to climb to temperatures near that of the air in the dryer (Christiansen 1990). This is the stage when typical wood surface inactivation occurs (Suchsland and Stevens 1968). 
Susceptibility to surface inactivation is prevalent in the drying of softwood species. For the most sensitive American coniferous species, severe surface inactivation occurs at the drying temperature of $160^{\circ} \mathrm{C}$ and higher (Christiansen 1990). Wood surfaces often experience this level of temperature during the drying of veneers, wood flakes, and wood particles in the wood-based composites industry, where high inlet drying temperatures up to $400^{\circ} \mathrm{C}$ are necessary for efficient and economical drying. Many experiments have shown that high drying temperature reduces the wood adhesive bond strength, or that high temperature decreases wood hygroscopicity and hinders wettability (Christiansen 1990; Kajita and Skaar 1992; Podgorsk et al. 2000).

\subsubsection{Objectives}

The objectives of this study were to determine the effect of temperature exposure on wood surface inactivation for hardwood yellow-poplar (Liriodendron tulipifera) and softwood southern pine (Pinus taeda). For that purpose, changes in the surface chemistry of wood due to temperature exposure were studied. The relationships among the chemistry, wettability, and adhesion of the wood surfaces in relation to temperature were also investigated. 


\subsection{Materials}

Heartwood samples of yellow-poplar (YP), and southern pine (SP) were studied. Both wood species had green MC above FSP. However, these initial MCs were different in respect to the fact that average MC varies considerably among species (Table 3.1). Only wood without gross defects was chosen for sample preparation. The samples were cut into lamellas and then planed to the thickness of $8 \mathrm{~mm}$ (Figure 3.1).
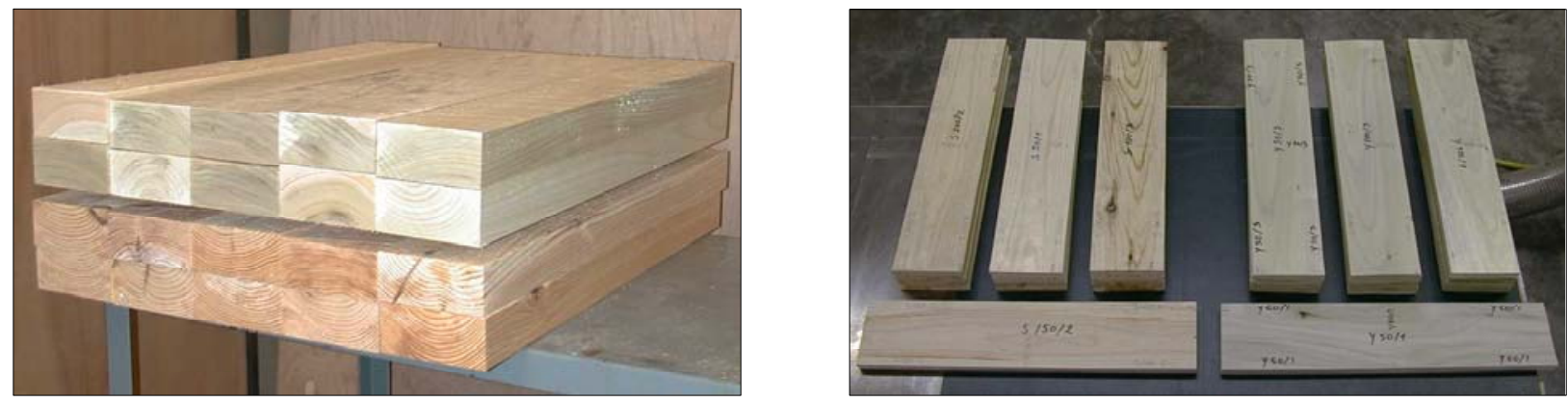

Figure 3.1. The machining of the wood samples: timber (left), lamellae (right).

\subsubsection{Heat Treatment - Drying of Wood Samples}

Wood samples were sorted in to five groups and then each group (i.e., three lamellas) was exposed to different drying conditions for identification of a critical temperature that causes surface inactivation. The samples from both wood species were dried together at selected drying parameters (Table 3.1).

Table 3.1. Properties of wood samples and drying parameters.

\begin{tabular}{|c|c|c|c|c|c|}
\hline \multirow{2}{*}{$\begin{array}{c}\text { Wood Properties and } \\
\text { Drying Parameters }\end{array}$} & \multicolumn{5}{|c|}{ Set Point Temperature $\left({ }^{0} \mathrm{C}\right)$} \\
\hline & 50 & 100 & 150 & 175 & 200 \\
\hline Initial Average MC (\%) of YP & 66.9 & 58.4 & 66.2 & 63.4 & 67.3 \\
\hline Initial Average $\mathrm{MC}(\%)$ of SP & 83.2 & 57.2 & 79.8 & 77.2 & 96.8 \\
\hline Max. Surface Temperature $\left({ }^{0} \mathrm{C}\right)$ & 51 & 104 & 156 & 172 & 187 \\
\hline Drying Time (hrs:min) & $17: 45$ & $05: 30$ & $02: 30$ & $01: 50$ & $02: 15$ \\
\hline Final Average MC (\%) & $1.5-2$ & $1.5-2$ & $1.5-2$ & $1.5-2$ & $1.5-2$ \\
\hline
\end{tabular}


Conventional drying in a convection oven was used to dry samples to a target MC of $2 \%$. This low final MC was chosen for two reasons: (1) inactivation occurs in the final stage of drying when MC is low, and (2) the XPS technique requires MC close to $0 \%$. The actual MC was controlled by the weight measurement of the samples during drying. Surface temperature of one lamella was monitored by a thermocouple. The temperature of the wood surface increased during drying in regard to the set point temperature and wood MC (Figure 3.2).

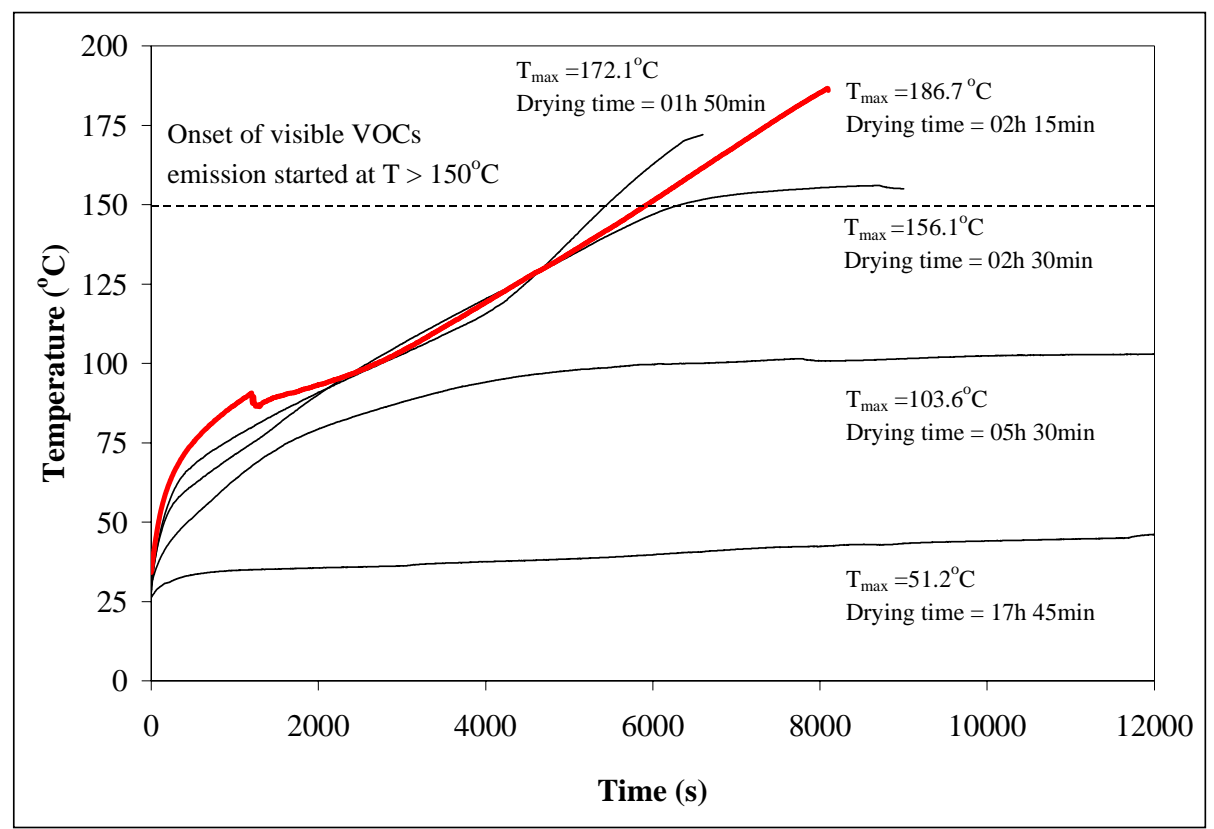

Figure 3.2. The increase of the wood surface temperature during drying.

Drying at lower temperature required a longer drying time, except for the drying temperatures of $175^{\circ} \mathrm{C}$, where the drying process took 25 minutes less than at $200^{\circ} \mathrm{C}$, because of the lower average initial MC. The emission of volatile organic compounds (VOCs) was recognized for the three highest set point temperatures. For these samples, wood started to release VOCs when the surface temperature reached $130^{\circ} \mathrm{C}$. The intensity of VOCs emission increased with temperature. 
An occurrence of VOCs emission, which appeared as smoke, was recognized when the surface temperature was above $150^{\circ} \mathrm{C}$. At this temperature, the $\mathrm{MC}$ was below $10 \%$, which is a typical MC when VOCs emission occurs ( $\mathrm{Su}$ et al. 1999). For instance, VOCs from dried particles increase sharply beyond $160^{\circ} \mathrm{C}$ (Banerjee et al. 1998), which is shown in Figure 3.3. VOCs emission is especially acute for softwoods, whose emission are primarily terpenes, exceed those from hardwood by an order of magnitude (Su et al. 1999).

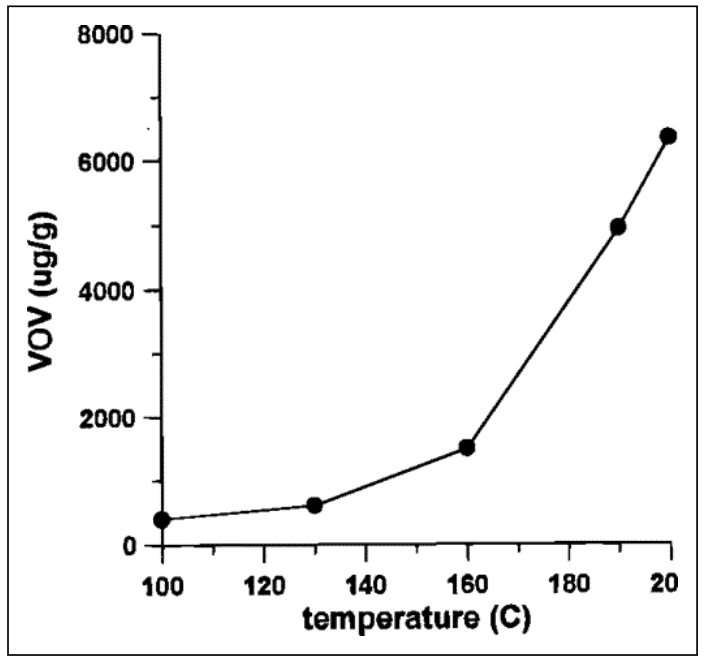

Figure 3.3. VOCs emission from dried particle at various temperatures (Banerjee et al. 1998). A vertical axis is $\operatorname{VOC}(\mu \mathrm{g} / \mathrm{g})$.

After drying, the samples were cooled to room temperature. Each lamella was cut into individual specimens for different study purposes (Figure 3.4). Special attention was given to ensure a clear and uncontaminated surface.

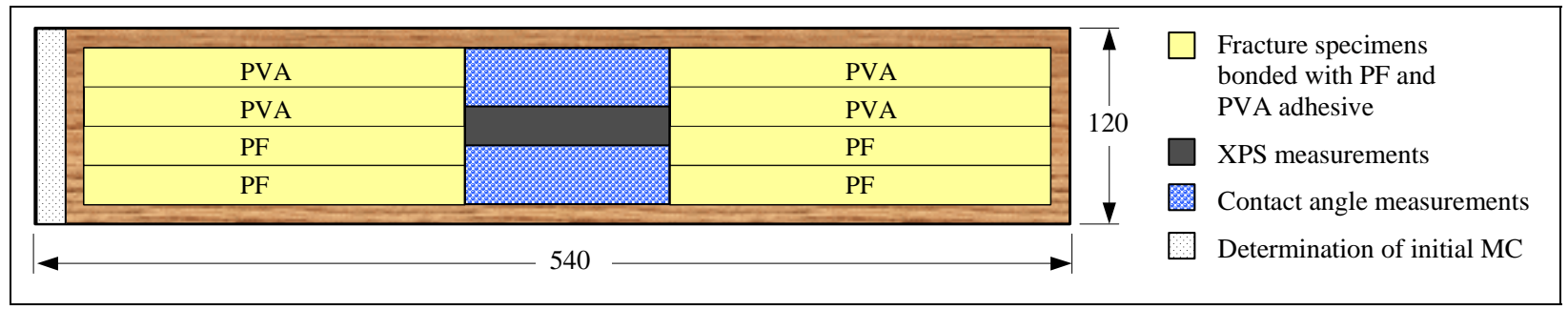

Figure 3.4. Specimen cutting diagram for each lamella. Width $(\mathrm{mm})$ is tangential direction. 


\subsubsection{Adhesives}

Thermoset phenol formaldehyde adhesive (PF) and thermoplastic polyvinyl acetate adhesive (PVA) were used to bond together two wood surfaces (Table 3.2).

Table 3.2. Specification of the adhesives.

\begin{tabular}{lccc}
\hline $\begin{array}{l}\text { Adhesive } \\
\text { property }\end{array}$ & $\begin{array}{c}\text { Phenol-formaldehyde adhesive } \\
\text { Chembond } ® \text { CB 303 }\end{array}$ & & $\begin{array}{c}\text { Polyvinyl-acetate adhesive } \\
\text { KOR LOK® GT 42-300A }\end{array}$ \\
\cline { 1 - 1 } Physical state & Liquid & Liquid \\
Solids content $(\%)$ & 47.1 & 51.4 \\
pH value & 10.5 & 3.3 \\
Specific gravity & 1.2 & 1.1 \\
Boiling point $\left({ }^{\circ} \mathrm{C}\right)$ & $\sim 100$ & $>100$ \\
Freezing point $\left({ }^{\circ} \mathrm{C}\right)$ & 0 & $<5$ \\
\hline
\end{tabular}

One half of each specimen was used with each adhesive, which produced 120 fracture specimens ( 2 wood species, 2 adhesives, 5 drying temperatures, and 6 replications). Adhesives were applied to one surface with a roller using a spreading level of $200 \mathrm{~g} / \mathrm{m}^{2}$. Assembly time was one minute. The PF bonded assemblies were cured in a laboratory press at $200^{\circ} \mathrm{C}$ and $2 \mathrm{~N} / \mathrm{mm}^{2}$ for 12 minutes. The PVA bonded assemblies were pressed with $2 \mathrm{~N} / \mathrm{mm}^{2}$ at $20^{\circ} \mathrm{C}$ for one hour. All bonded assemblies were then stored in plastic bags for $24 \mathrm{hrs}$ at room temperature.

\subsection{Methods}

\subsubsection{X-Ray Photoelectron Spectroscopy}

A Perkin-Elmer model 5400 X-ray photoelectron spectrometer was employed to provide elemental and chemical data of the wood surface composition. The tangential wood surface with an area of $8 \times 5 \mathrm{~mm}$ and with a thickness of $3 \mathrm{~mm}$ was studied. In total, 30 measurements were obtained (i.e., 2 wood species, 5 drying temperatures, and 3 replications). 
The wood sample was fixed on a sample holder by double stick tape and then put in the chamber. The sample was exposed to the vacuum and cooling. The purpose of a low pressure and a low temperature was to slow molecular motions of the air, which minimized the influence of air molecules on the results. When a pressure of $<5 \times 10^{-7}$ torr was achieved, an X-ray source was activated. X-rays were irradiated from $\mathrm{Mg} \mathrm{K} \alpha(1253.6 \mathrm{eV})$ with an incident angle of $45^{\circ}$.

A wood surface area of approximately $3 \mathrm{~mm}^{2}$ was observed, and a surface depth $<50 \AA$ was analyzed. The relative concentration of the detected elements was determined. The ratio of the elements was calculated based on the atomic sensitivity factor and on the area under each peak of the spectra for the elements in question.

\subsubsection{Contact Angle Measurement}

A sessile drop method was used to measure a contact angle $(\theta)$ of a $7.5 \mu \mathrm{l}$ distilled water drop, which was applied to the surface by means of a digital pipette. The contact angle was defined as the angle formed between the surface of a solid and the line tangent to the droplet radius from the point of contact with the solid. Since the tendency for the liquid to spread on the surface increases as $\theta$ decreases, determination of $\theta$ is a useful inverse measure of wettability (Zisman 1964). A $\cos \theta$ often serves as a direct measure of wettability (Kajita and Skaar 1992).

The image of the liquid drop was captured by a video camera and transferred to a computer screen (Figure 3.5) where the contact angle was measured by digital image analysis software (ImagePro, Media Cybernetics). The image was captured immediately after the water drop was applied ( 0 seconds), and then every 10 seconds for a duration of one minute. In total, 420 measurements were performed (i.e., 2 wood species, 5 drying temperatures, 7 times in one minute, and 6 replications). 


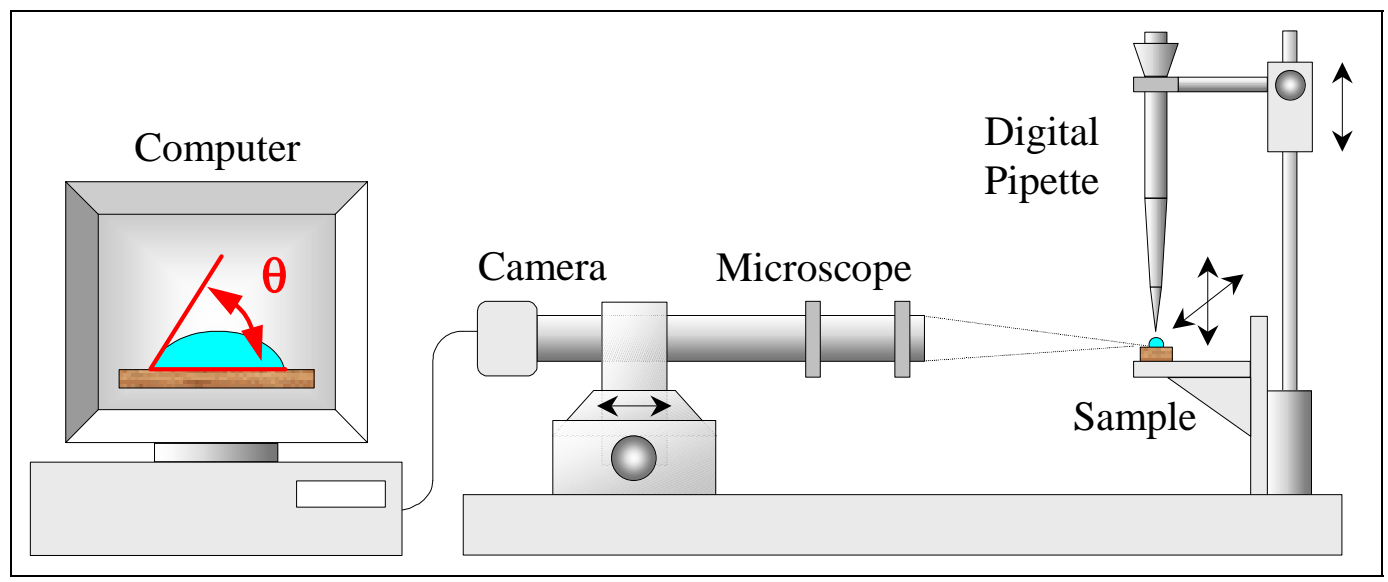

Figure 3.5. The contact angle equipment set-up.

\subsubsection{Fracture Mechanics Test}

A fracture test in cleavage/opening mode (Mode I) was employed to obtain the critical strain energy release rate $\left(\mathrm{G}_{\mathrm{C}}\right)$, which is a measure of the energy required to fracture the adhesive bond and to create a new surface (Schmidt 1998). The double cantilever beam (DCB) geometry was used, which is one of the most popular test specimen geometries for measuring adhesive fracture energy (Blackman et al. 1991). The DCB test method may be conducted to measure the fracture energy of a bonded joint, which is influenced by adherend surface condition, adhesiveadherend interactions, etc. (ASTM D3433 1997).

Two improvements (Gagliano and Frazier 2001; Hashemi et al. 1990) were adopted for specimen preparation and for data analysis: a flat double cantilever beam (DCB) specimen, and a shear corrected compliance method. The flat double cantilever beam geometry greatly simplifies sample preparation. The shear corrected compliance method accounts for variations in wood modulus, and it corrects a crack length measurement due to shear effects in wood adherends.

The test specimens were prepared according to defined procedures by Schmidt (1998), with some modifications in dimensions specific to this study. The length of the specimens was oriented in the longitudinal direction, and in a way which kept crack propagation within the bondline. The grain angle was $5-10^{\circ}$. A saw was used to create a $40 \mathrm{~mm}$ pre-crack in one end of the bondline, which was additionally modified by a knife to ensure a sharp crack tip (Figure 3.6). 


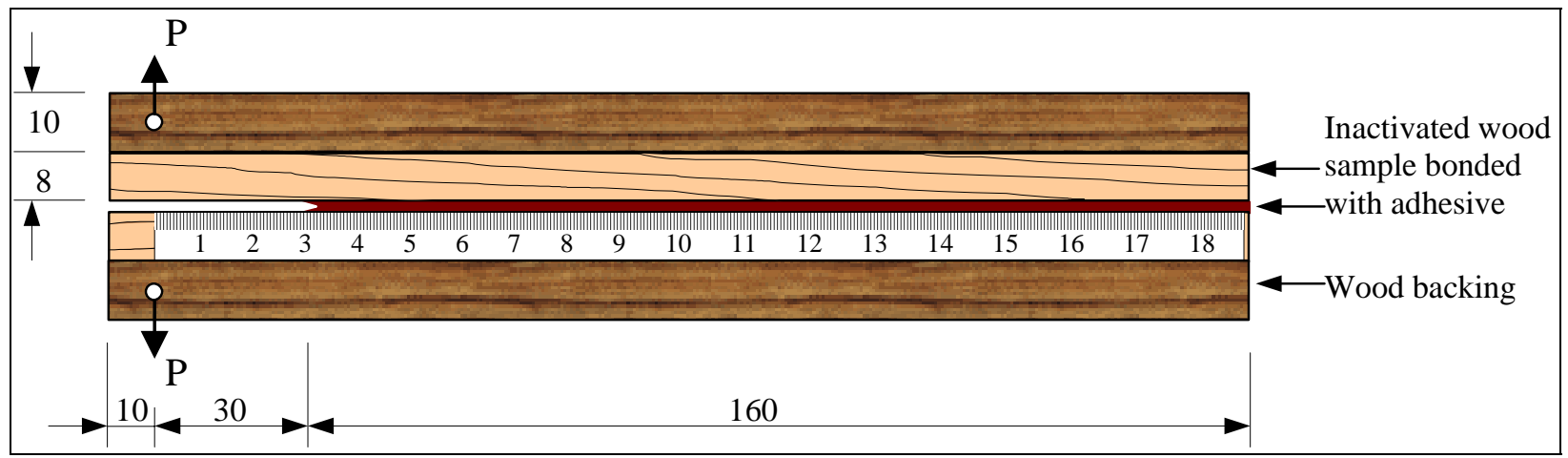

Figure 3.6. Geometry and dimensions $(\mathrm{mm})$ of the fracture test specimen.

As seen in Figure 3.6, an additional backing was needed because otherwise the specimens would be too thin for the satisfactory attachment to the test grips. In order to fix the specimen into machine grips, two holes needed to be drilled in the middle of the each lamella's thickness and $10 \mathrm{~mm}$ from the edge. Consequently, the drilled holes in the $16 \mathrm{~mm}$ thick specimen would be $8 \mathrm{~mm}$ apart. However, the distance between the holes has to be more than $8 \mathrm{~mm}$ to fit in the grip, since the hole in the grip is $4 \mathrm{~mm}$ from the edge (Figure 3.7).

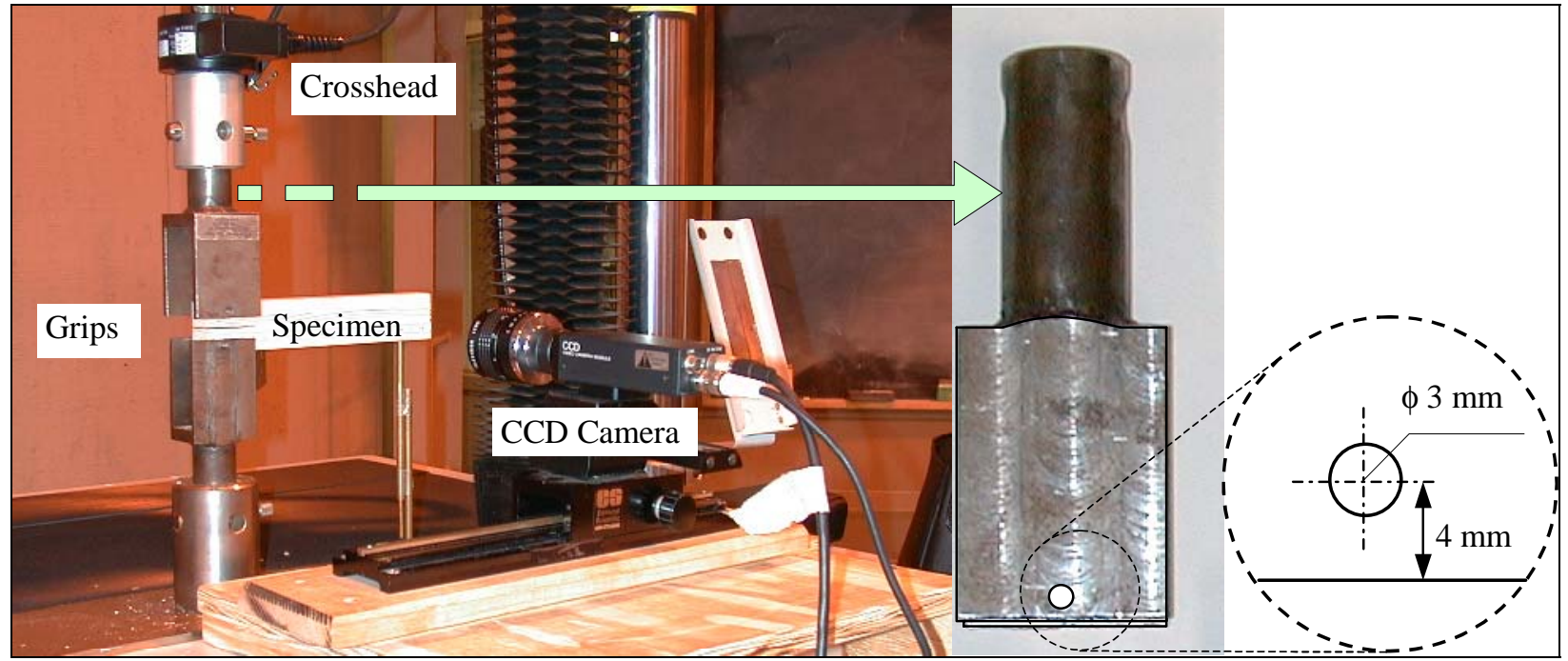

Figure 3.7. Fracture test setup showing a mounted specimen and the specimen grip. 
When the two holes are less than $8 \mathrm{~mm}$ apart, it is not possible to position both grips close enough to insert the specimen. Therefore, a $10 \mathrm{~mm}$ thick wood lamella was bonded with PVA adhesive to both sides of the bonded assemblies. The holes, which were needed for attachment to the test fixture, were drilled into these lamellas. These additional parts did not influence the measurements, since the shear corrected compliance method does not depend (within the assumptions) on the cross-sectional area of the test specimen.

The same PVA adhesive as described previously was used to bond the backing to the specimen. The adhesive was applied by means of a roller at the spreading level of $200 \mathrm{~g} / \mathrm{m}^{2}$. The assembly time was one minute. The adhesive was cured at room temperature in a laboratory press at a low pressure of $0.1 \mathrm{~N} / \mathrm{mm}^{2}$ for one hour. Two $20 \mathrm{~mm}$ wide specimens were then cut from one bonded assembly (discarding the periphery), yielding six fracture specimens per sample. Prior to testing, the specimens were conditioned for two weeks at $20( \pm 2)^{\circ} \mathrm{C}$ and at relative humidity of $65( \pm 2) \%$. For the crack length measurement, the adhesive bondline was painted with white typographic correction fluid to accurately indicate the crack tip in the fractured zone. The crack length is the distance between the loading point and the crack tip.

The fracture testing was performed using a screw-driven universal testing machine (MTS) in a displacement control mode. One end of the specimen was placed in the test apparatus while the free end was supported to maintain horizontal placement. A 5 Newton load was applied to the sample, and then the crosshead position was zeroed. Loading $(\mathrm{P})$ was initiated at $1 \mathrm{~mm} / \mathrm{min}$ displacement and it was applied until a crack initiated and propagated. That caused the load to decrease. When the load dropped more than 3\%, the displacement was held constant until the crack was arrested (45 sec.). This allowed the crack to arrest and the load to become quasi-stable (Gagliano 2001). The crosshead was then returned to zero displacement and the whole procedure was repeated. The specimen failed catastrophically after several repeated cycles.

The displacement rate for each subsequent loading cycle was increased so that a fracture occurs within one minute. The first displacement rate was set to be $1 \mathrm{~mm} / \mathrm{min}$. The displacement rates for each subsequent cycle were calculated by dividing the maximum opening displacement from the previous cycle by one minute. This ensures that the crack tip strain rate remains constant as the DCB lever arms extend with crack growth (Gagliano and Frazier 2001). 
The crack length was measured manually by using the scale applied along the length of the specimen. A video camera with 10x magnification was used to assure precise measurement of the specimen crack length displayed on a video monitor. Specimen loads (i.e., maximum load and arrested load) and crosshead positions (i.e., displacement) were recorded using the computer data acquisition system TestWorksTM (Figure 3.8).

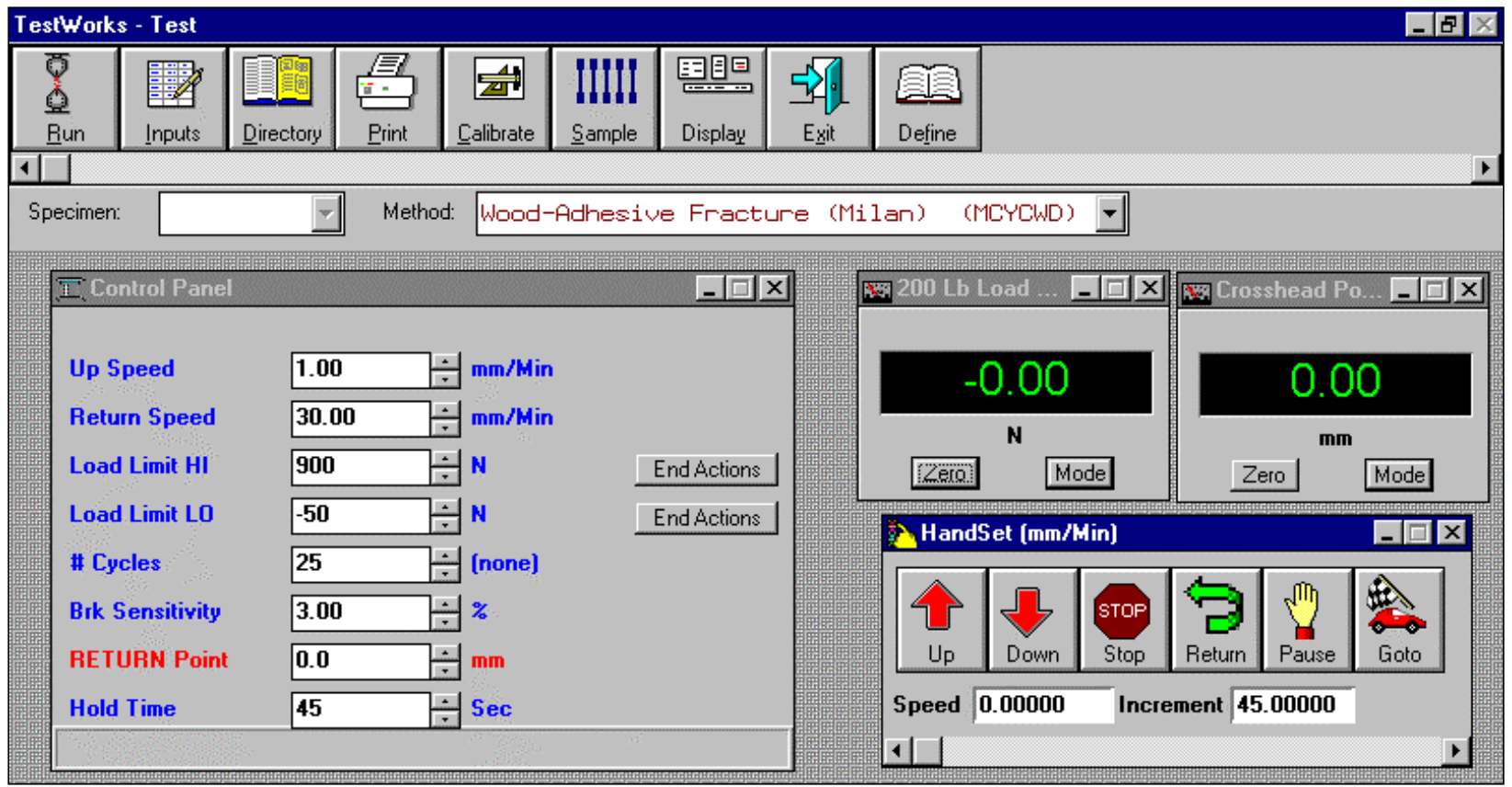

Figure 3.8. TestWorks ${ }^{T M}$ data acquisition system with the parameters setup for fracture test.

Each specimen provided 4 to 10 data points, which were used to calculate the maximum and arrested strain energy release rates. Since shear forces were present at very short distances from a loading point, and the assumptions of a beam on an elastic foundation were not valid at long distances, only data obtained on the specimen's length from 50 to $150 \mathrm{~mm}$ was used (Gagliano 2001). The evaluation of the measurements was carried out with the corrected compliance method (Gagliano and Frazier 2001). The equations (2.10, 2.11, and 2.12) derived by Hashemi et al. (1990) were used. 


\subsubsection{Adhesive Penetration}

The adhesive penetration was investigated on the samples heat-treated at 51 and $187{ }^{\circ} \mathrm{C}$ and bonded with PF adhesive. Both wood species were included. The measurements were performed on microscope slide sections, which were taken from the cross-sectional surface of the YP50, YP200, SP50, and SP200 specimens.

Before cutting the microscope sections, the specimens $(30 \times 10 \times 10 \mathrm{~mm})$ were immersed in water and exposed for 30 minutes to a vacuum $(20 \mathrm{~mm} \mathrm{Hg})$, followed by 30 minutes of exposure to atmospheric pressure. This cycle was repeated until the specimens were completely saturated with water. The $20 \mu \mathrm{m}$ thick sections where then cut using a sliding microtome (White et al. 1997). To ensure a greater precision of measurements, the microscopic sections were colored for 24 hours by immersion in a $0.5 \%$ aqueous solution of the coloring agent Toluidine Blue O. The sections were then repeatedly washed in distilled water until the water remained clear. This was followed by dehydration in $70 \%$, and $100 \%$ ethanol. After drying at room temperature, the sections were fixed between a microscopic slide and a cover glass by using glycerin. Six microscope slide sections were prepared and two replicate measurements were done on each section, providing 12 data points per sample.

$\mathrm{PF}$ adhesive penetration was measured using an epi-fluorescence microscope (Zeiss Axioskop), a 100W HBO lamp, and digital image analysis technique (Johnson and Kamke 1992). The surface area of $1.66 \mathrm{~mm}^{2}$, including the adhesive bond, was randomly selected and captured as a digital image. A manual process, called thresholding, was used to isolate the adhesive for measurement on the digital image. After completing the selection, the computer program (ImagePro ${ }^{\mathrm{TM}}$ ) calculated the statistical parameters for all of the selected adhesive objects. Adhesive penetration was quantified as maximum penetration (MP) and effective penetration (EP) (Sernek et al. 1999). The EP is the total area (A) of the adhesive detected in the interphase region of the bondline divided by the width $\left(\mathrm{x}_{0}\right)$ of the bondline:

$$
E P=\frac{\sum_{1}^{n} A_{i}}{x_{0}}
$$


The MP is the average distance of the five most distant adhesive objects detected within the field of view:

$$
M P=\frac{\sum_{1}^{5}\left(y_{i}+r_{i}-y_{0}\right)}{5}
$$

\section{Equation 3.2}

$A_{i}$ is an area of a single object $\left(\mu \mathrm{m}^{2}\right), y_{i}$ is a centroid of the five objects representing the deepest penetration $(\mu \mathrm{m}), \mathrm{r}_{\mathrm{i}}$ is the mean radius of an object $(\mu \mathrm{m})$, and $\mathrm{y}_{0}$ is a coordinate of the bondline $(\mu \mathrm{m})$. A graphical explanation of these parameters is shown in Figure 3.9.

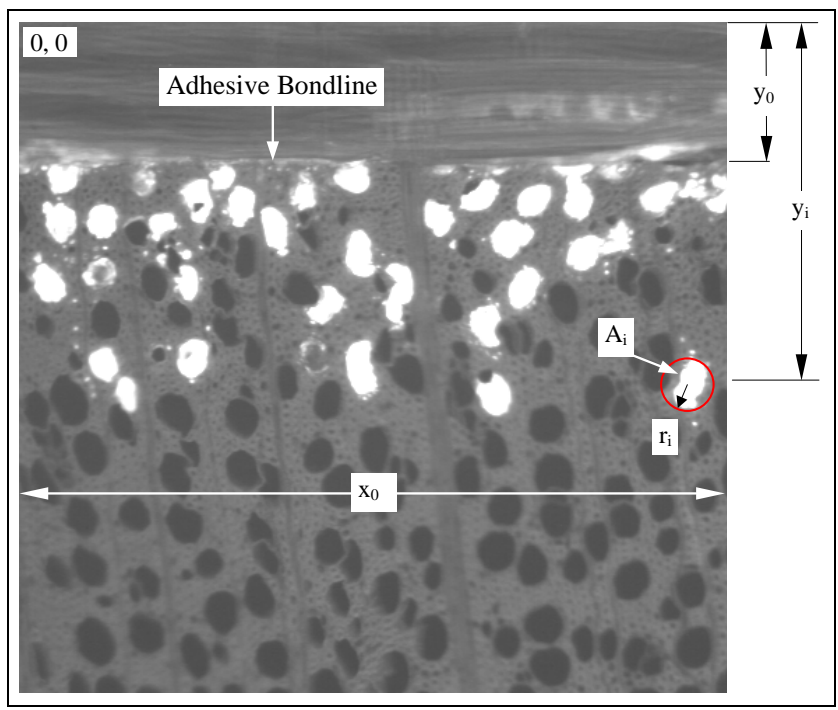

Figure 3.9. Measurement parameters used in calculating EP and MP.

\subsubsection{Statistical Observations}

All the measurements obtained in this study and following studies were statistically evaluated and the hypotheses were tested. Descriptive statistics, such as an average value (AVERAGE), standard deviation (STDEV), covariance (COV), and sample size (n), are listed in the appendices. A linear multiple regression, analysis of variance, t-test, and Duncan multiple range tests were employed (Johnson and Bhattacharyya 1992; Mann 1995). All estimations were based on 95\% confidence level. Statistical software STATGRAPH and EXCEL were used. 


\subsection{Results and Discussion}

\subsubsection{Influence of Drying Temperature on Chemical Changes of Wood Surface}

Carbon $(\mathrm{C} 1 \mathrm{~s})$, oxygen $(\mathrm{O} 1 \mathrm{~s})$, and nitrogen $(\mathrm{N} 1 \mathrm{~s})$ elements were detected on the investigated surfaces. The surfaces also contained hydrogen $(\mathrm{H})$, but this element cannot be detected by the XPS technique. A typical wide scan XPS spectrum (i.e., survey spectrum) is shown in Figure 3.10.

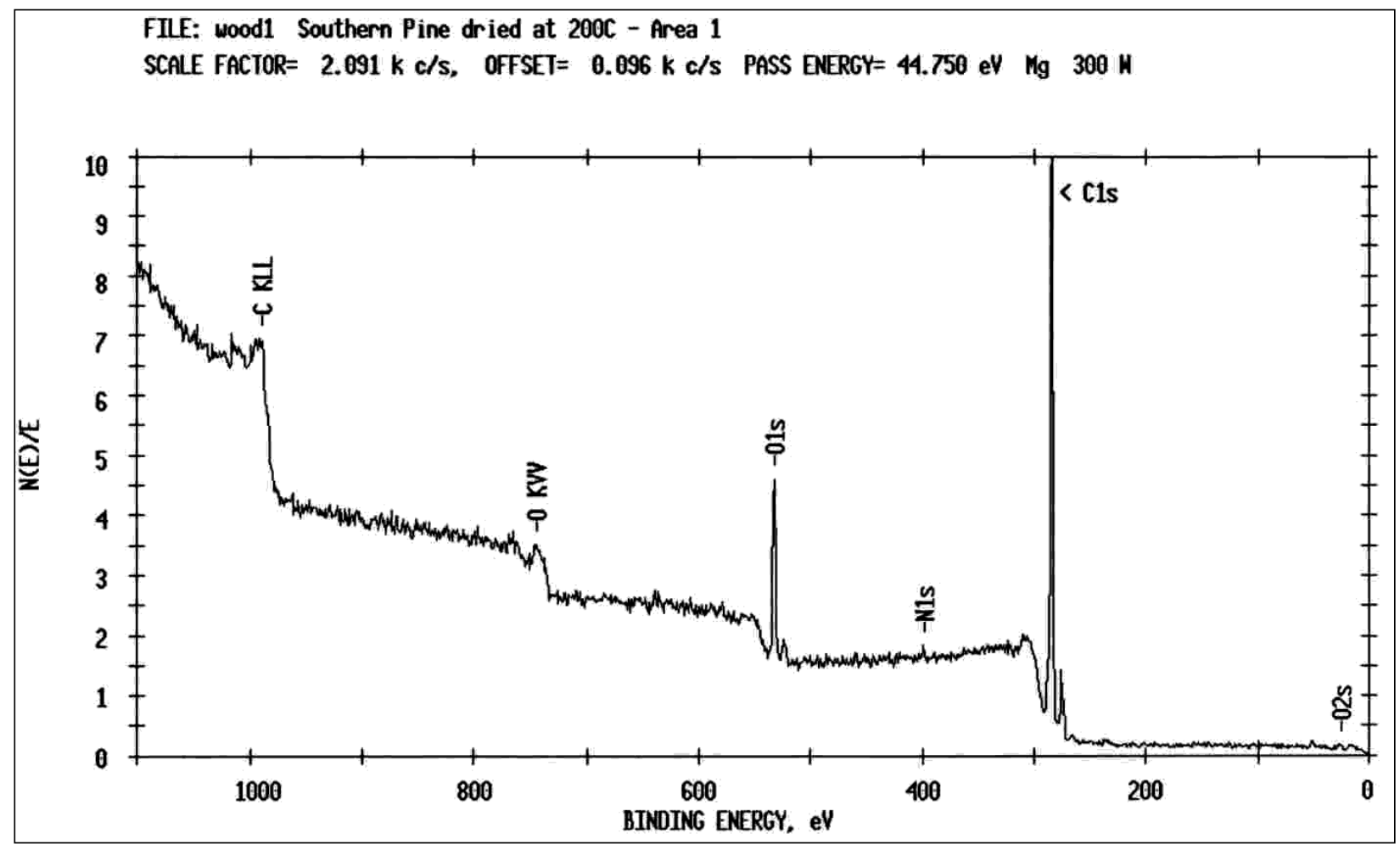

Figure 3.10. Wide scan XPS spectrum for southern pine surface exposed to $200^{\circ} \mathrm{C}$.

The ratio of the elements, which was calculated by using the atomic sensitivity factor and the curve area under each peak for the detected element, was determined from the XPS measurements. This allowed expressing the surface chemical composition by an atomic percent of the elements, which indicates the relative concentration of an element. 
The components of the carbon and oxygen atoms were determined from the deconvoluted spectra, which is actually an enlargement of the binding energy scale of a peak obtained for the carbon and oxygen elements. This procedure was followed by a computer curve fitting and by determining the binding energy shifts for $\mathrm{C} 1 \mathrm{~s}$ and $\mathrm{O} 1 \mathrm{~s}$. The curve fit spectra were derived based on the assumption of a normal probability function assigned to the each of the three carbon or oxygen components (PHI-ESCA, Version 4.0B, PHI Division of Perkin-Elmer).

The curve fit carbon $\mathrm{C} 1$ s peak was comprised of three main components: $\mathrm{C} 1, \mathrm{C} 2$, and C3 (Figure 3.11). The curve fit oxygen O1s peak was comprised of O1, O2, and O3 components (Figure 3.12). The components of carbon and oxygen atoms are related. The O1 type of oxygen is bonded to $\mathrm{C} 3$ type of carbon in $\mathrm{C}=\mathrm{O}$ bonds. The $\mathrm{O} 2$ type of oxygen is connected with $\mathrm{C} 2$ type of carbon as $\mathrm{C}-\mathrm{O}$ bond. The assignment for $\mathrm{O} 3$ type of oxygen is not explainable. Its peak might be broadening by hydrogen bonding effects in wood.

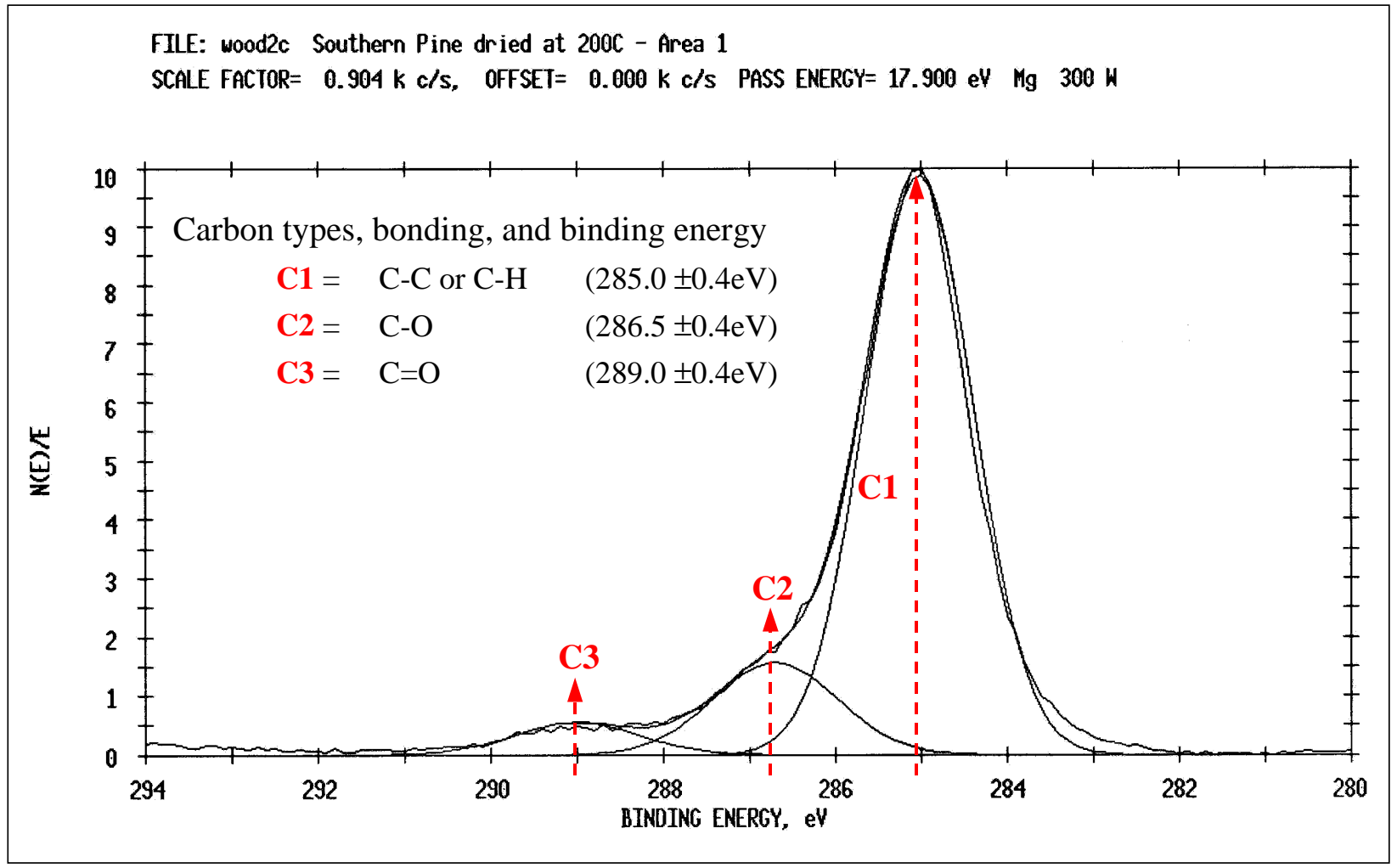

Figure 3.11. Curve fits of carbon Cls peak of southern pine surface exposed to $200{ }^{\circ} \mathrm{C}$. 
FIIE: MS1260 SOUTHERN PINE 200

SCALE FACTOR $=0.433 \mathrm{k} \mathrm{c} / \mathrm{s}$, OFFSET $=0.000 \mathrm{k} \mathrm{c} / \mathrm{s}$. PASS ENERGY $=17.900 \mathrm{eV} \mathrm{Mg} 300 \mathrm{~K}$

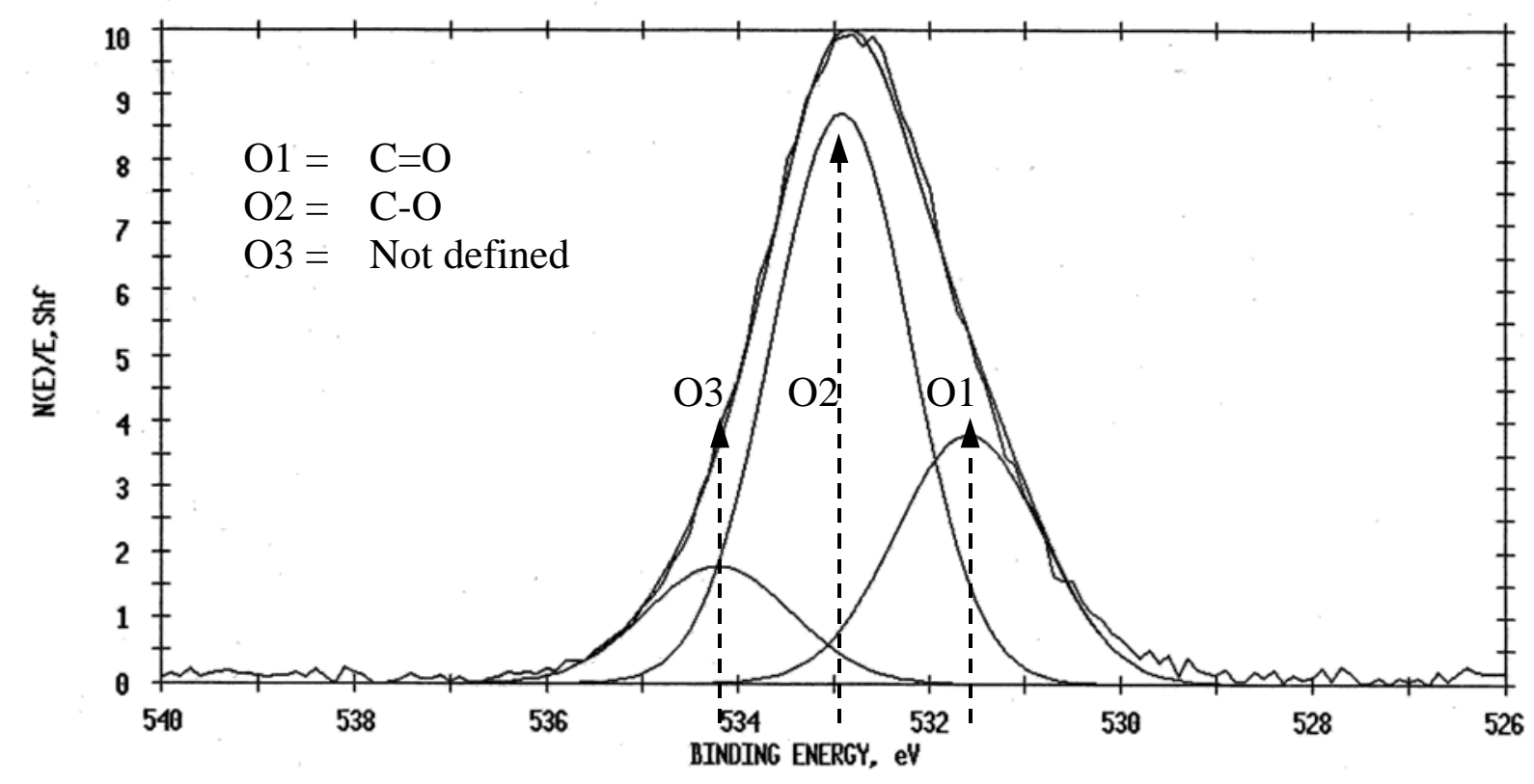

Figure 3.12. Curve fits of Ols peak of southern pine surface exposed to $200^{\circ} \mathrm{C}$.

The atomic percent of the detected elements is shown in Table 3.3 and Table 3.4. The results of all XPS measurements and descriptive statistics are presented in Appendix A.

Table 3.3. Atomic percents of yellow-poplar surfaces determined by XPS.

\begin{tabular}{|c|c|c|c|c|c|c|c|c|c|c|}
\hline \multicolumn{2}{|c|}{ Yellow-Poplar } & \multicolumn{9}{|c|}{ Atomic Percent } \\
\hline \multirow{2}{*}{$\begin{array}{c}\text { Sample } \\
\text { Name }\end{array}$} & \multirow{2}{*}{$\begin{array}{l}\text { Maximum Surface } \\
\text { Temperature }\left({ }^{\circ} \mathbf{C}\right)\end{array}$} & \multicolumn{3}{|c|}{ Elements } & \multicolumn{3}{|c|}{ C1s Components } & \multicolumn{3}{|c|}{ O1s Components } \\
\hline & & C1s & $01 \mathrm{~s}$ & N1s & C1 & $\mathrm{C} 2$ & $\mathbf{C 3}$ & 01 & $\mathrm{O} 2$ & $\mathbf{O 3}$ \\
\hline YP50 & 51 & 75.3 & 24.2 & 0.5 & 60.7 & 32.4 & 7.0 & 11.8 & 78.3 & 9.9 \\
\hline YP100 & 104 & 75.9 & 23.5 & 0.6 & 60.4 & 31.7 & 8.0 & 10.4 & 79.5 & 10.1 \\
\hline YP150 & 156 & 74.8 & 24.5 & 0.7 & 59.4 & 32.0 & 8.6 & 17.4 & 73.2 & 9.4 \\
\hline YP175 & 172 & 78.5 & 20.7 & 0.8 & 63.9 & 28.7 & 7.4 & 24.8 & 66.4 & 8.8 \\
\hline YP200 & 187 & 81.3 & 18.3 & 0.3 & 72.4 & 22.2 & 5.4 & 31.3 & 59.5 & 9.2 \\
\hline
\end{tabular}


Table 3.4. Atomic percents of southern pine surface as determined by XPS.

\begin{tabular}{|c|c|c|c|c|c|c|c|c|c|c|}
\hline \multicolumn{2}{|c|}{ Southern Pine } & \multicolumn{9}{|c|}{ Atomic Percent } \\
\hline \multirow{2}{*}{$\begin{array}{l}\text { Sample } \\
\text { Name }\end{array}$} & \multirow{2}{*}{$\begin{array}{l}\text { Maximum Surface } \\
\text { Temperature }\left({ }^{\circ} \mathbf{C}\right)\end{array}$} & \multicolumn{3}{|c|}{ Elements } & \multicolumn{3}{|c|}{ C1s Components } & \multicolumn{3}{|c|}{ O1s Components } \\
\hline & & C1s & 01s & N1s & C1 & C2 & $\mathrm{C3}$ & 01 & 02 & $\mathbf{O 3}$ \\
\hline SP50 & 51 & 80.2 & 19.2 & 0.6 & 73.9 & 17.8 & 8.3 & 14.8 & 61.4 & 23.8 \\
\hline SP100 & 104 & 80.2 & 19.1 & 0.7 & 72.9 & 19.1 & 8.0 & 14.6 & 64.2 & 21.2 \\
\hline SP150 & 156 & 84.2 & 15.4 & 0.5 & 78.1 & 15.7 & 6.3 & 15.2 & 61.9 & 22.9 \\
\hline SP175 & 172 & 84.0 & 15.6 & 0.4 & 78.8 & 14.8 & 6.4 & 13.5 & 62.5 & 24.0 \\
\hline SP200 & 187 & 85.2 & 14.3 & 0.5 & 77.9 & 15.8 & 6.4 & 19.0 & 60.2 & 20.7 \\
\hline
\end{tabular}

The changes in atomic percent (i.e., C1s, O1s, and N1s) showed that the drying temperature affected the chemical composition of wood surfaces. The percent of carbon increased with drying temperature, and consequently, the percent of oxygen decreased with drying temperature. The percent of nitrogen did not change much and it was below $1 \%$. These trends were obtained for the yellow-poplar samples and for the southern pine samples.

The Duncan multiple range test (95\% confidence level) was used to identify statistically significant differences among the samples. The analyses showed that the concentration of carbon and oxygen for YP samples exposed to $187^{\circ} \mathrm{C}$ were significantly different from samples dried at the lower three temperatures $\left(51,104\right.$, and $\left.156{ }^{\circ} \mathrm{C}\right)$. SP samples exhibited a quite different relationship—samples exposed to the three higher temperatures $\left(156,172\right.$, and $\left.187{ }^{\circ} \mathrm{C}\right)$ had significantly different contents of carbon and oxygen than those exposed to lower temperatures.

Besides the atomic percent, the oxygen to carbon ratio $(\mathrm{O} / \mathrm{C}$ ratio) and the $\mathrm{C} 1 / \mathrm{C} 2$ ratio were calculated. Both ratios are related to the chemical composition of wood constituents, which allows for the identification of the principal components on the wood surface (i.e., polysaccharides, lignin, and extractives). Since only three replicate measurements were conducted, all the data points were represented on the following four graphs. A solid line presents an average value. Figure 3.13 and Figure 3.14 show the influence of drying temperature on the total $\mathrm{O} / \mathrm{C}$ ratio of yellow-poplar and southern pine, respectively. 


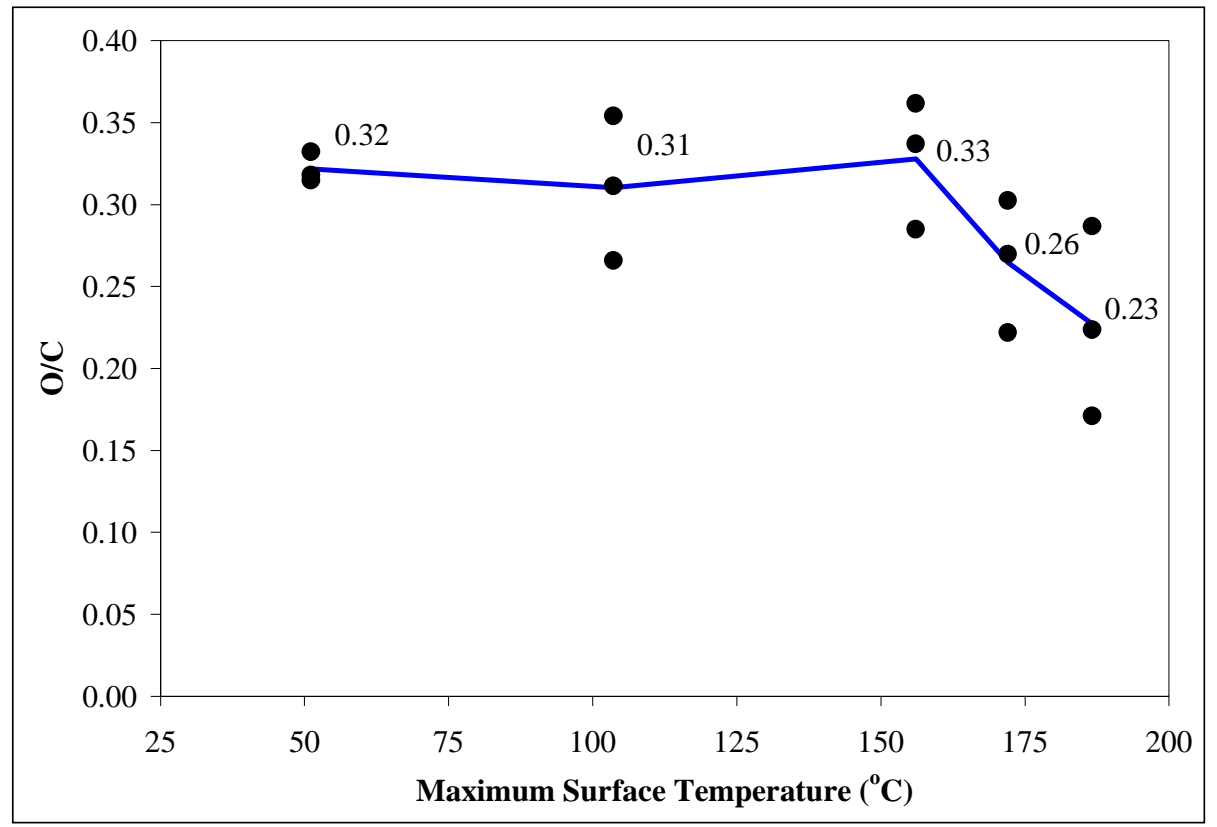

Figure 3.13. The influence of drying temperature on the O/C atomic ratio of yellow-poplar.

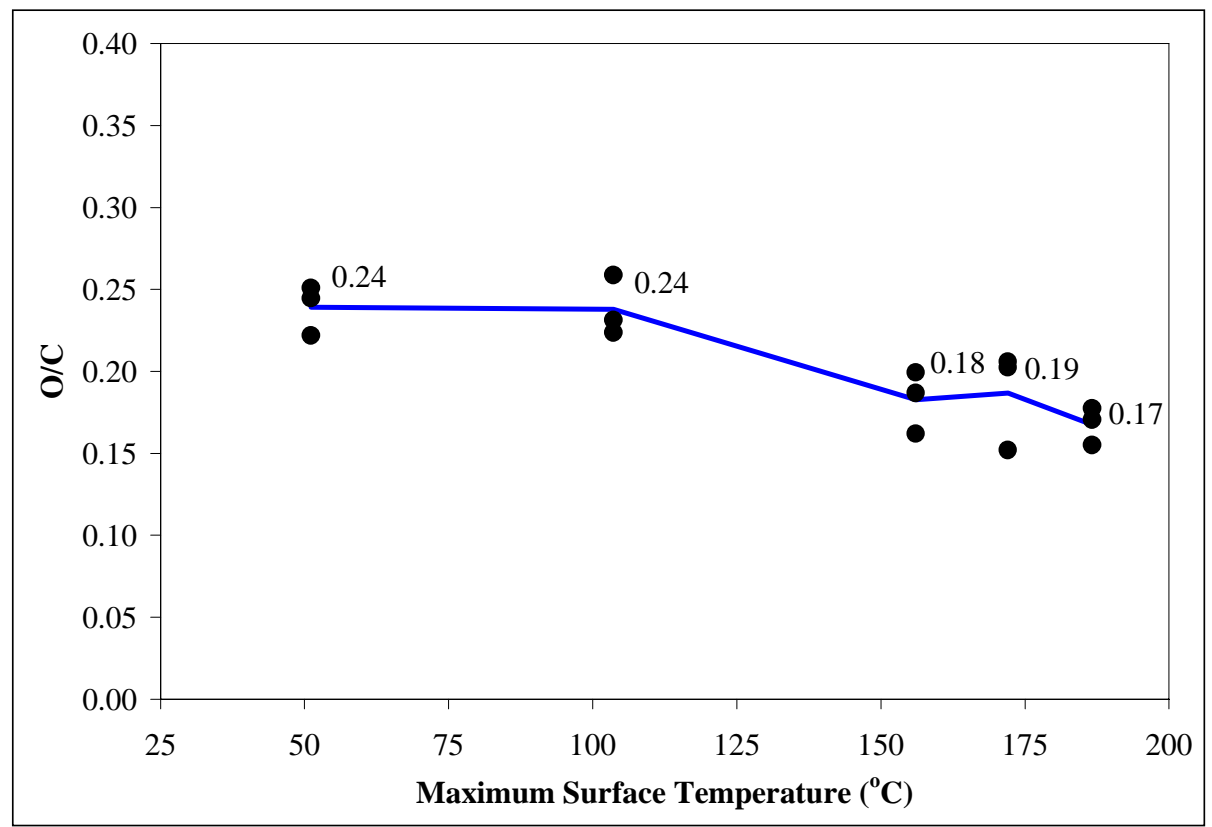

Figure 3.14. The influence of drying temperature on the O/C atomic ratio of southern pine. 
The $\mathrm{O} / \mathrm{C}$ ratio of $\mathrm{YP}$ stayed quite constant up to the drying temperature of $156^{\circ} \mathrm{C}$. The average $\mathrm{O} / \mathrm{C}$ value (i.e., solid line in Figure 3.13) on this interval was 0.32. At a higher drying temperature, the $\mathrm{O} / \mathrm{C}$ ratio decreases, dropping to the value of 0.23 at $187^{\circ} \mathrm{C}$. Statistical analysis showed a significant difference between this $\mathrm{O} / \mathrm{C}$ value compared to those $\mathrm{O} / \mathrm{C}$ ratios obtained at all three lower drying temperatures. The influence of drying temperature on the average $\mathrm{O} / \mathrm{C}$ ratio of SP became significant earlier, at the drying temperature of $156^{\circ} \mathrm{C}$, and remained quite constant at the higher drying temperatures. Values of the $\mathrm{O} / \mathrm{C}$ ratio obtained at the temperatures of 51 and $104^{\circ} \mathrm{C}$ were significantly different than those obtained at the drying temperature of $156^{\circ} \mathrm{C}$ and higher. In all cases, SP exhibited a lower O/C ratio than YP. This is consistent with the results in previous studies (Ben et al. 1993; Börås and Gatenholm 1999).

According to the theory, a high $\mathrm{O} / \mathrm{C}$ ratio represents a surface containing mostly polysaccharides. A low $\mathrm{O} / \mathrm{C}$ ratio reflects a high concentration of extractives and lignin on the wood surface. The theoretical value of $\mathrm{O} / \mathrm{C}$ ratio for cellulose is 0.83 ; while for lignin and extractives it is much lower at 0.33 and 0.10, respectively (Barry et al. 1990). Therefore, the results indicated that the SP wood surface (up to a depth of $50 \AA$ ) should contain a higher amount of extractives and lignin than the YP wood surface. More precisely, it can be assumed that SP wood surfaces contained a higher amount of resinous extractives than YP wood surfaces. SP resins are mainly comprised of acidic diterpenoids (Stanley 1969), which have a low O/C ratio. For instance, abietic acid has the $\mathrm{O} / \mathrm{C}$ ratio of 0.10 (Börås and Gatenholm 1999).

Calculation of the $\mathrm{C} 1 / \mathrm{C} 2$ ratio provided additional evidence in support of the $\mathrm{O} / \mathrm{C}$ interpretation of the wood surface chemistry. The components represent different chemical bonding states of carbon. The $\mathrm{C} 1$ component is related to carbon-carbon and carbon-hydrogen bonds in extractives and lignin. The bonds involving C2 can result from all three classes of wood components, but predominantly in the carbohydrates as $-\mathrm{CHOH}$ and in lignin as $\beta$-ether and $-\mathrm{C}$ $\mathrm{OH}$ bonds. $\mathrm{C} 3$ carbon atoms occur as carbonyl groups of the lignin and as the carbon atom bonded to two oxygen atoms of polysaccharides (Young et al. 1982). The calculated theoretical $\mathrm{C} 1 / \mathrm{C} 2$ ratio for pure cellulose is 0 , for lignin close to 1 , and for extractives around 10 or higher. Figure 3.15 and Figure 3.16 show the influence of drying temperature on the $\mathrm{C} 1 / \mathrm{C} 2$ ratio of yellow-poplar and southern pine, respectively. 


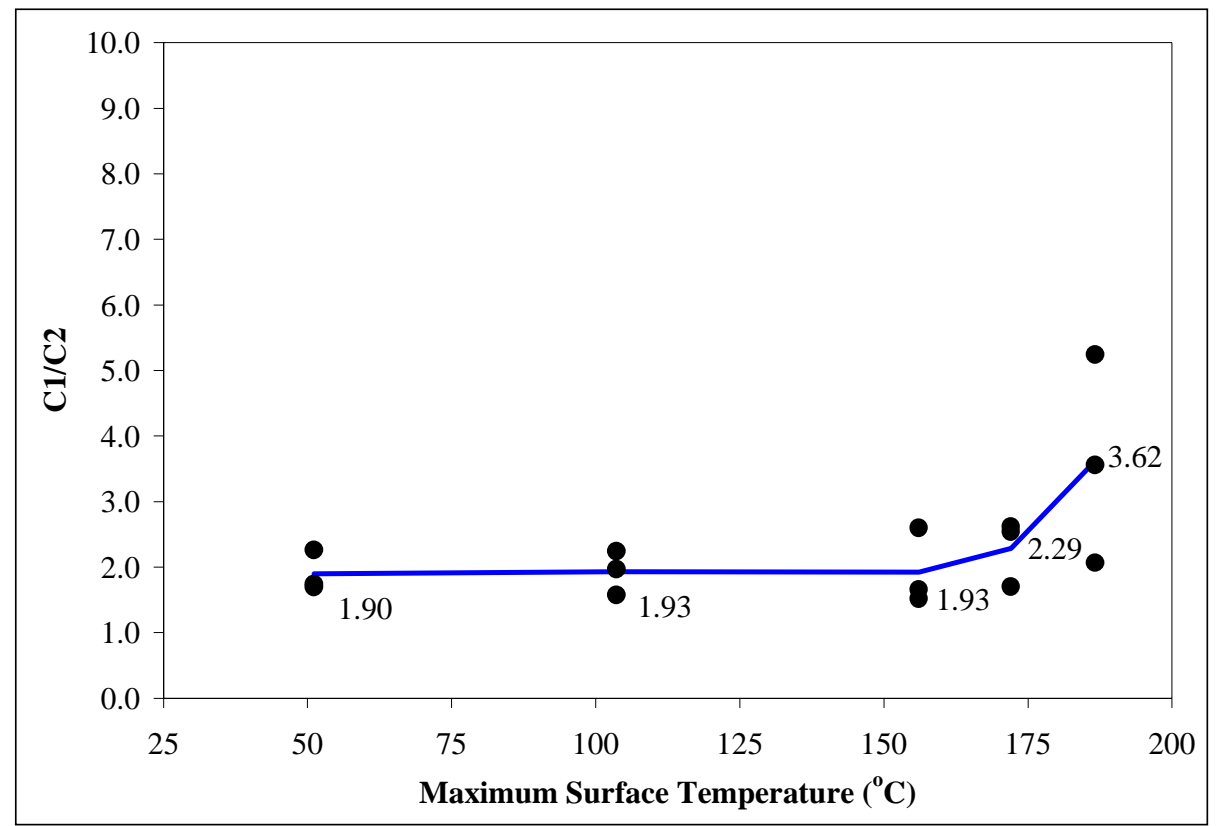

Figure 3.15. The influence of drying temperature on the C1/C2 atomic ratio of yellow-poplar.

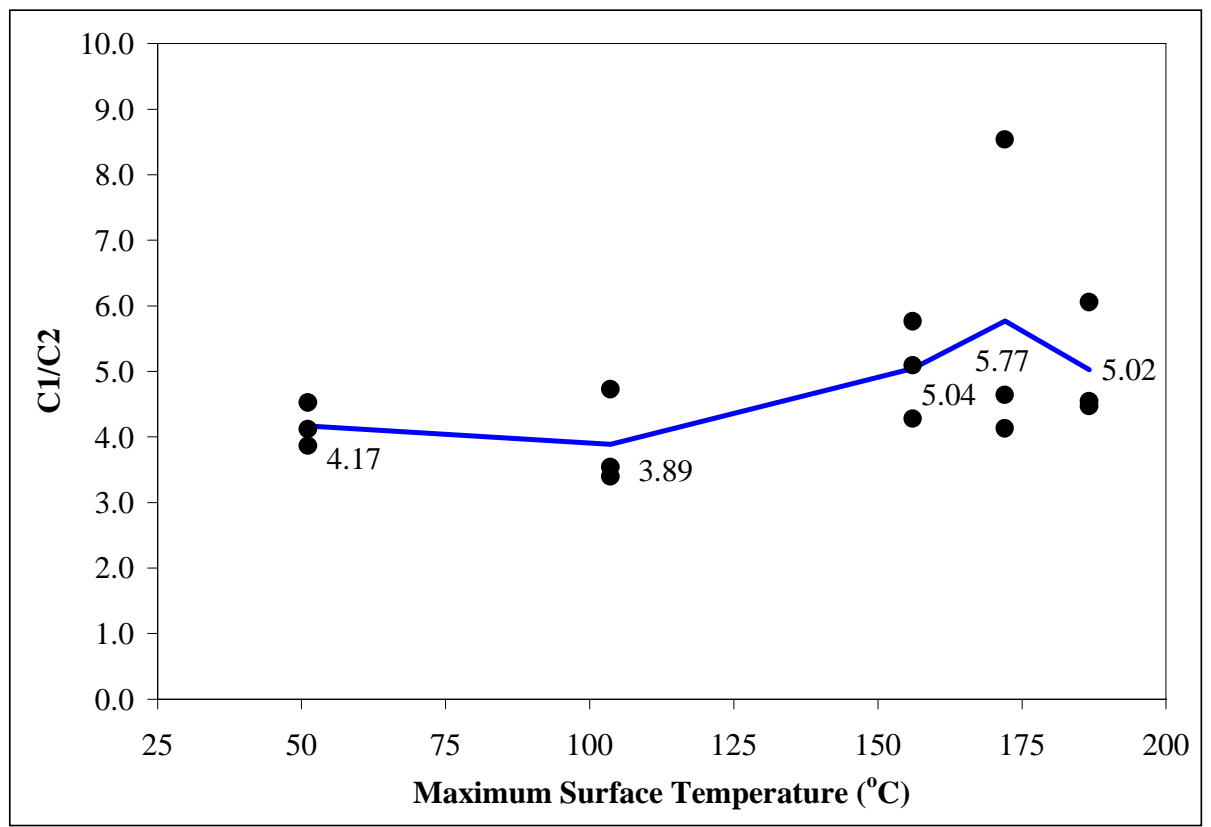

Figure 3.16. The influence of drying temperature on the C1/C2 atomic ratio of southern pine. 
The average $\mathrm{C} 1 / \mathrm{C} 2$ ratio of $\mathrm{YP}$ stayed constant up to the drying temperature of $156^{\circ} \mathrm{C}$, and then started to increase with temperature. A significant jump to an average value of 3.62 was obtained at a drying temperature of $187^{\circ} \mathrm{C}$. Samples of SP did not exhibit significant differences in the $\mathrm{C} 1 / \mathrm{C} 2$ ratios regarding different temperature exposures, but a trend of increasing $\mathrm{C} 1 / \mathrm{C} 2$ ratio with drying temperature was obtained. A comparison of the average $\mathrm{C} 1 / \mathrm{C} 2$ ratios between YP and SP showed that all the C1/C2 ratios of SP are higher than the C1/C2 ratios of any YP samples. Since the surface content of hydrophobic material can be expressed as the $\mathrm{C} 1 / \mathrm{C} 2$ ratio (Börås and Gatenholm 1999), this result suggested that SP surfaces contained higher amounts of hydrophobic extractives and lignin than YP surfaces. The same conclusion emerged from the $\mathrm{O} / \mathrm{C}$ ratio results, where $\mathrm{SP}$ exhibited lower $\mathrm{O} / \mathrm{C}$ ratios than $\mathrm{YP}$.

It can be summarized that increasing drying temperature made wood surfaces more hydrophobic, possibly because of the migration of extractives to the surface. The quantity of extractives transported to the surface depends mainly on relative humidity and temperature. The relative humidity affected the moisture gradient, which promoted mass flow. Increased temperature accelerated water movement. Water-soluble extractives were transported to the wood surface along with water during the drying operation. Water-insoluble extractives might migrate to the wood surface in a vapor phase at high drying temperatures (Hse and Kuo 1988).

The changes in surface chemistry can also be ascribed to some rearrangement of lignin at the surface. This was possible when the temperature of the wood surface exceeded the glass transition temperature $\left(\mathrm{T}_{\mathrm{g}}\right)$ of lignin. The $\mathrm{T}_{\mathrm{g}}$ of dry lignin in wood is $65-105^{\circ} \mathrm{C}$, and it decreases with increasing wood MC (Glasser 2000). Temperatures higher than $\mathrm{T}_{\mathrm{g}}$ promote polymer mobility and allow rearrangement of molecules. It is known that polymer surfaces are time-, temperature-, and environment-dependent (Gunnells et al. 1994). Molecules of the polymer surface can reorient to present a low energy surface to the air. The driving force for reorientation is thermodynamic; a surface tends to minimize its free energy. Since amorphous and glassy polymers (e.g., lignin and hemicelluloses in wood) are not in thermodynamic equilibrium, they tend to rearrange. 
It seems that the migration of extractives to the wood surface is a dominant mechanism, which explains the changes in the wood surface chemistry in regard to temperature exposure. However, this explanation needs a precise consideration. One can see that the $\mathrm{O} / \mathrm{C}$ ratio of southern pine drops significantly at temperatures $>156^{\circ} \mathrm{C}$. This is the temperature when excessive VOC emissions start to occur. During the samples' drying in this study, an appearance of smoke was recognized when the surface temperature $>150^{\circ} \mathrm{C}$, which indicated that significant chemical changes occur. Thus, emission of VOCs, their degradation, and some possible deposition on the wood surface, might have an impact on surface inactivation. The emission of VOCs and their possible influence on wood surface inactivation through pyrolytic degradation of all or selected wood components, is discussed in the next chapter.

It is questionable how to elucidate changes in the $\mathrm{O} / \mathrm{C}$ and the $\mathrm{C} 1 / \mathrm{C} 2$ ratios in terms of inactivation. One might conclude only that inactivated wood surfaces exhibit lower $\mathrm{O} / \mathrm{C}$ ratios and higher $\mathrm{C} 1 / \mathrm{C} 2$ ratios than active wood surfaces (i.e., freshly produced). It is meaningless to interpret the $\mathrm{O} / \mathrm{C}$ and $\mathrm{C} 1 / \mathrm{C} 2$ ratios as inactivation indicators by using absolute values, because these values can vary substantially among wood species as well as within a wood species. Therefore, an evaluation of relative changes in the wood surface chemistry provides more fruitful interpretation of inactivation.

Besides this, it is necessary to relate wood surface chemistry to the bonding performance, since the wood surface inactivation is also defined through aspects of adhesion. If there is a clear relationship between wood surface chemistry and adhesion, the expression through the O/C and $\mathrm{C} 1 / \mathrm{C} 2$ ratios can be used to elucidate wood surface inactivation from a chemical aspect.

\subsubsection{Influence of Drying Temperature on Wood Wettability}

The drying temperature affected wood surface wettability. The lowest contact angle of a water drop was obtained on the surfaces that were exposed to the lowest drying temperature of $51^{\circ} \mathrm{C}$, and the highest contact angle was obtained on the surfaces that were exposed to the highest drying temperature of $187^{\circ} \mathrm{C}$ (Figure 3.17). The results of contact angle measurements and descriptive statistics are presented in Appendix B and Appendix C. 

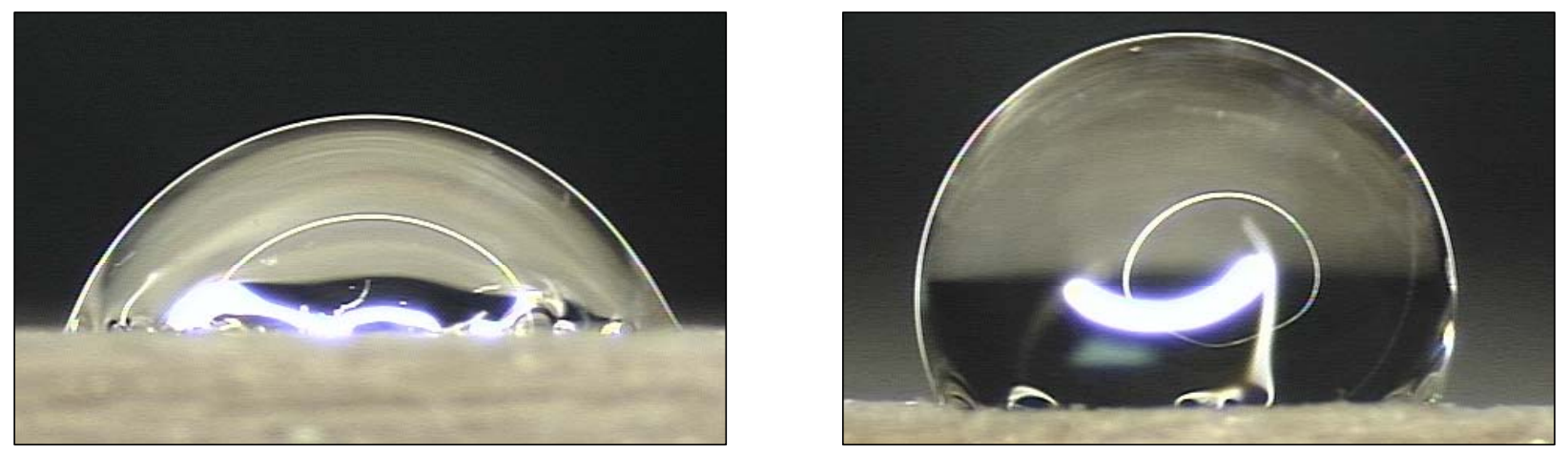

Figure 3.17. Typical initial contact angle of a water drop on the SP wood surface dried at $51{ }^{\circ} \mathrm{C}$ (left), and on the inactivated SP wood surface dried at $187^{\circ} \mathrm{C}$ (right).

For both wood species, the contact angle decreased with time, and increased with the drying temperature. However, it was significantly lower for the YP sample (Figure 3.18) compared with the SP sample (Figure 3.19). This relationship was expected, since high temperatures accelerate migration of extractives to the wood surface (USDA 1999). This increases the hydrophobic character of the wood surface. Wood hydrocarbon extractives are mostly hydrophobic, thus a surface that is rich with extractives repels water. Consequently, the hydrophobic surface exhibits a high contact angle and a low wettability. The surface of SP was more hydrophobic than YP. This was expected because SP contains a higher amount of extractives than YP, 3.5-5.4\% and 2.4-3.8\%, respectively (Rowe 1989; White 1987). Also, SP extractives comprise a high proportion of wood resins, including terpenes, (Fengel and Wegener 1989; Stanley 1969), which are all very hydrophobic. Additionally, yellow-poplar generates smaller amount of VOCs than southern pine. The VOC emission of SP is by an order of magnitude higher than hardwoods (Banerjee 2001). Moreover, SP contains more lignin than YP, $27 \%$ and 20\%, respectively (Pettersen 1984). Since lignin is a hydrophobic substance, its concentration on the wood surface causes lower wettability. 


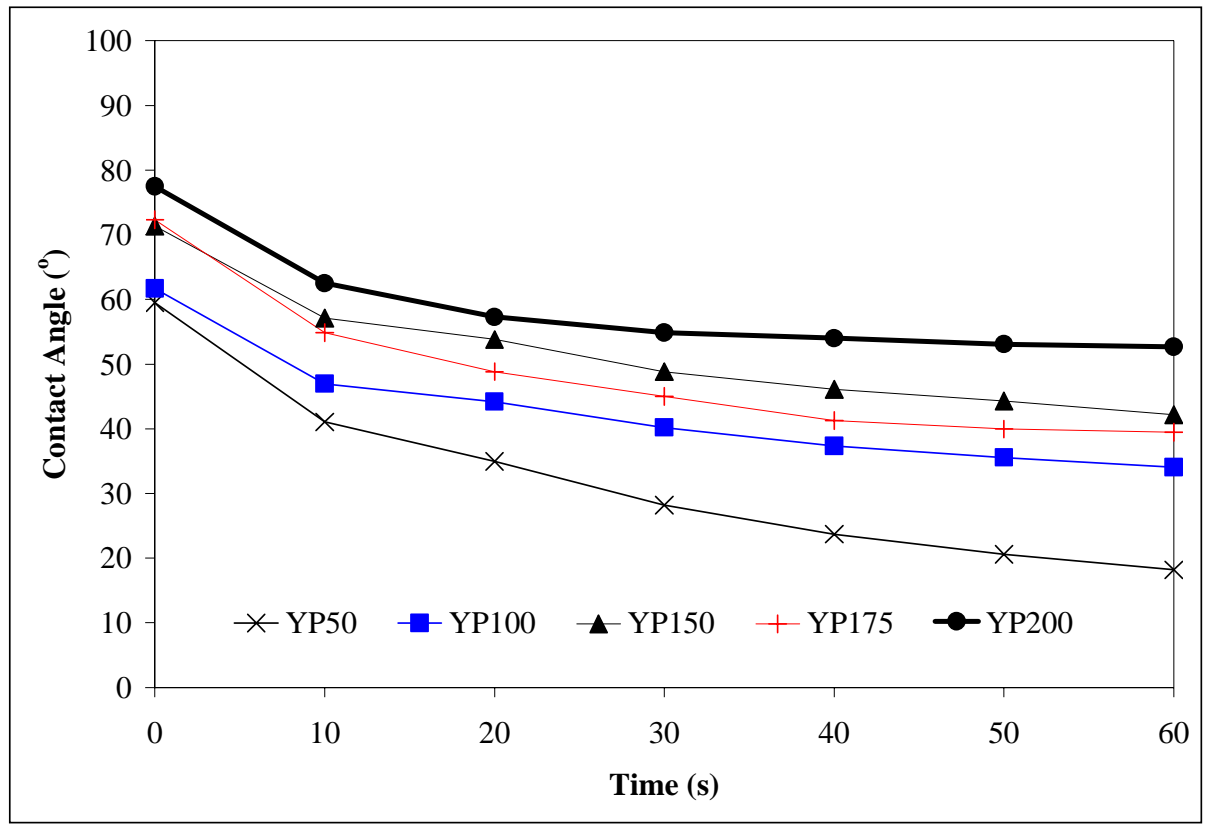

Figure 3.18. Time dependence of the contact angle for yellow-poplar.

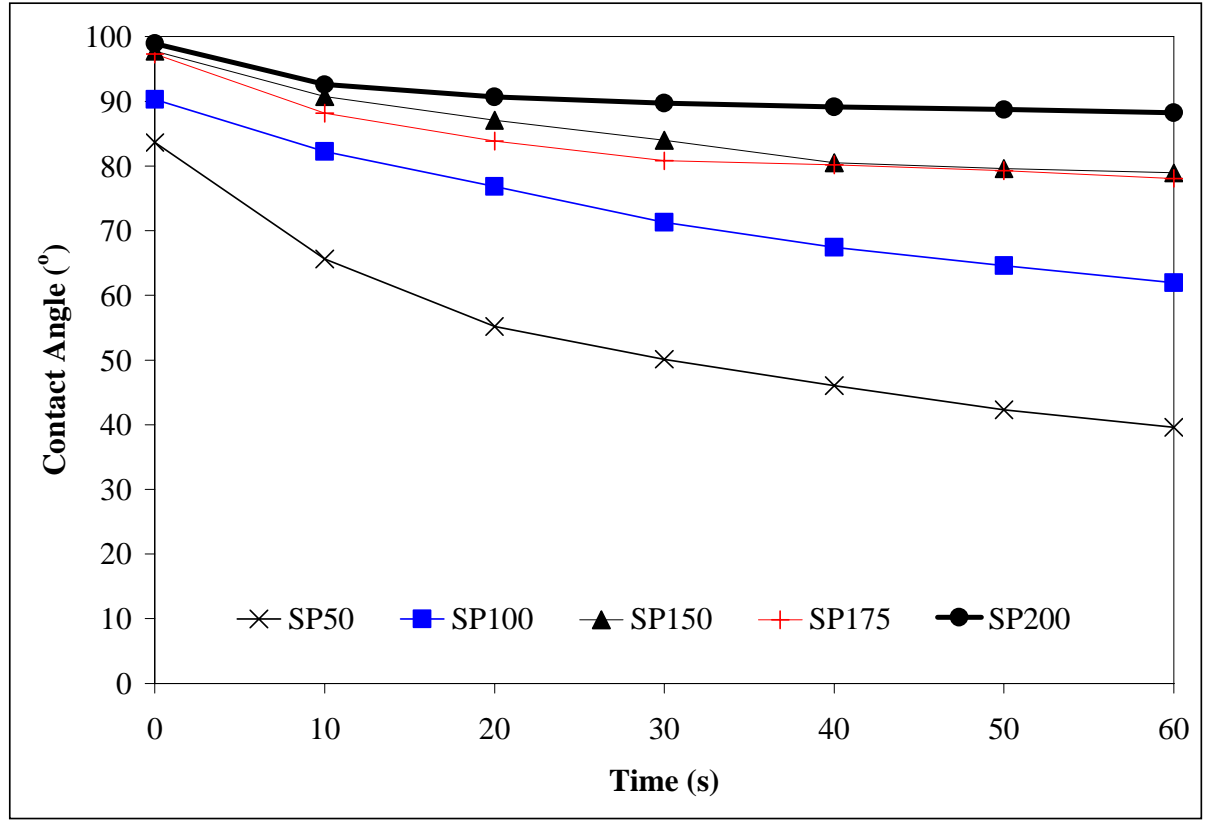

Figure 3.19. Time dependence of the contact angle for southern pine. 
The equilibrium condition as assumed in Young's equation cannot be achieved on wood surfaces. Therefore, the contact angle results are more often used as a relative value (i.e., to compare among wood species) than as a thermodynamic value. Chemical heterogeneity, surface roughness, and hygroscopicity of wood usually impede an establishment of an equilibrium contact angle (Gardner et al. 1991a). Porous and hygroscopic wood absorbs water into its structure; thus, the contact angle changes over time. Moreover, swelling of the wood surface (Wellons 1977) and contamination of the probe liquid with soluble wood extractives (Wålinder and Johansson 2001) also affect contact angle measurement.

Since the equilibrium condition cannot be achieved, the validity of the thermodynamic wettability principles for a wood surface is limited. But observing the time dependent behavior of a drop of water on the wood surface provides a good early indicator of how the water-borne adhesive might later behave. The rate of contact angle change $(\Delta \theta / \Delta t)$ was different in regard to the drying temperature (Figure 3.20).

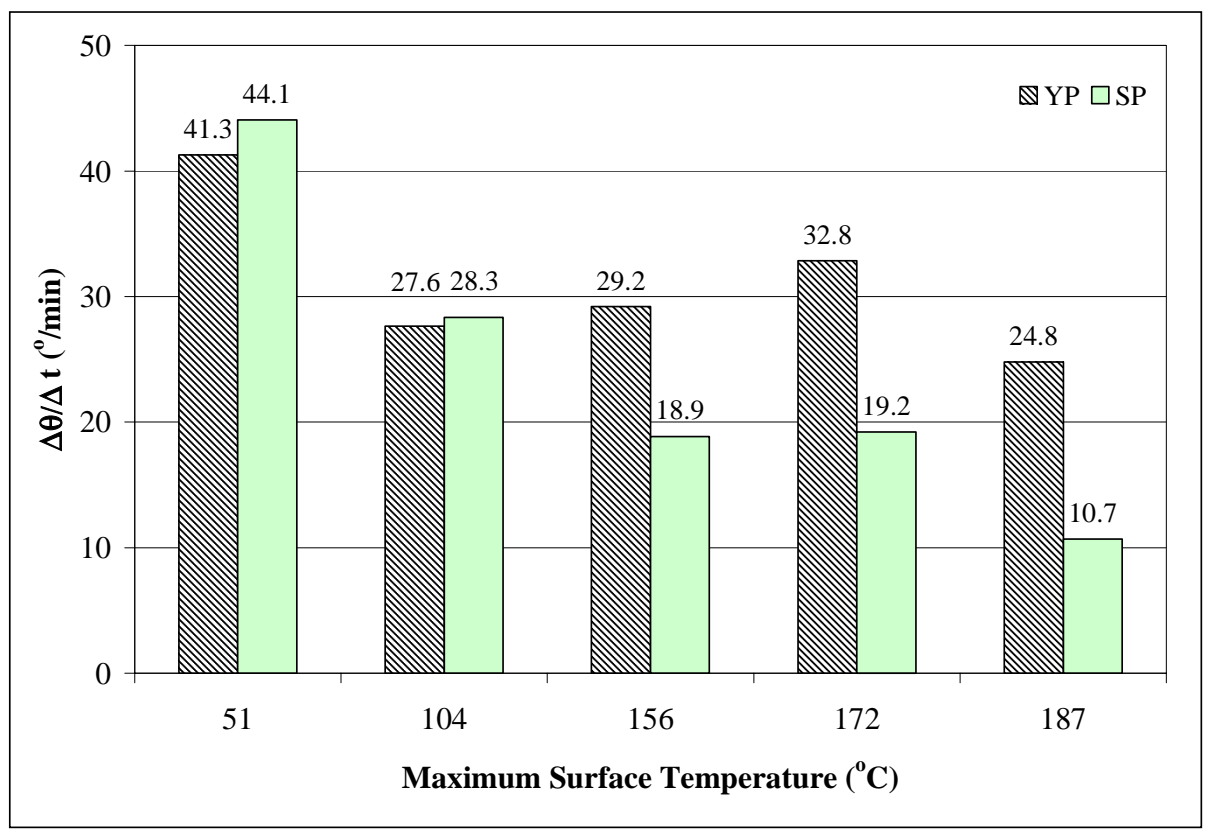

Figure 3.20. The rate of contact angle change during one minute in respect to drying temperature exposure. 
For both wood species, the biggest changes in the contact angle were observed at lower drying temperatures, and the smallest changes in the contact angle were observed at higher drying temperatures. Within one minute, the average contact angle obtained on surfaces dried at $51^{\circ} \mathrm{C}$ changed for $\mathrm{YP}$ and SP as much as $41.3^{\circ}$ and $44.1^{\circ}$, respectively. These changes were smaller when the contact angle was measured on YP and SP surfaces dried at $187^{\circ} \mathrm{C}$, with values of $24.8^{\circ}$ and $10.7^{\circ}$, respectively. The wood surface with the smallest change in the contact angle exhibited the lowest adhesive bond performance $\left(G_{\max }\right)$, which is shown later in section 3.4.3. Therefore, the changes in the rate of contact angle change are an indication of how strong adhesion will develop between surfaces.

SP exhibited quite different dependence of $\Delta \theta / \Delta t$ in regard to drying temperature than YP. The rate of contact angle change dropped significantly at $104^{\circ} \mathrm{C}$ and then it stayed almost unchanged. On the other hand, SP exhibited substantially lower $\Delta \theta / \Delta \mathrm{t}$ at drying temperatures higher than $156^{\circ} \mathrm{C}$ compared with $\Delta \theta / \Delta \mathrm{t}$ obtained on the surfaces dried at 51 and $104{ }^{\circ} \mathrm{C}$. Again, this indicates that VOCs may play important role in the inactivation phenomena at higher temperatures.

For evaluating the dynamics of the contact angle change, the absolute value of a rate of contact angle decline $(\Delta \theta / \Delta \mathrm{t})$ was calculated. The $\Delta \theta / \Delta \mathrm{t}$ is expressed as a fraction in the change of contact angle (in a time interval) divided by the time interval. For both wood species, the $\Delta \theta / \Delta t$ was the fastest at the beginning when the water drop was applied, and then it tended to level off (Figure 3.21 and Figure 3.22). However, the $\Delta \theta / \Delta t$ leveled off sooner for samples dried at $187^{\circ} \mathrm{C}$, while it continued to change for the samples dried at lower temperatures. SP exhibited less change in the $\Delta \theta / \Delta \mathrm{t}$ than YP. The SP specimens that were dried at $187^{\circ} \mathrm{C}$, exhibited the smallest changes in the $\Delta \theta / \Delta$ t. 


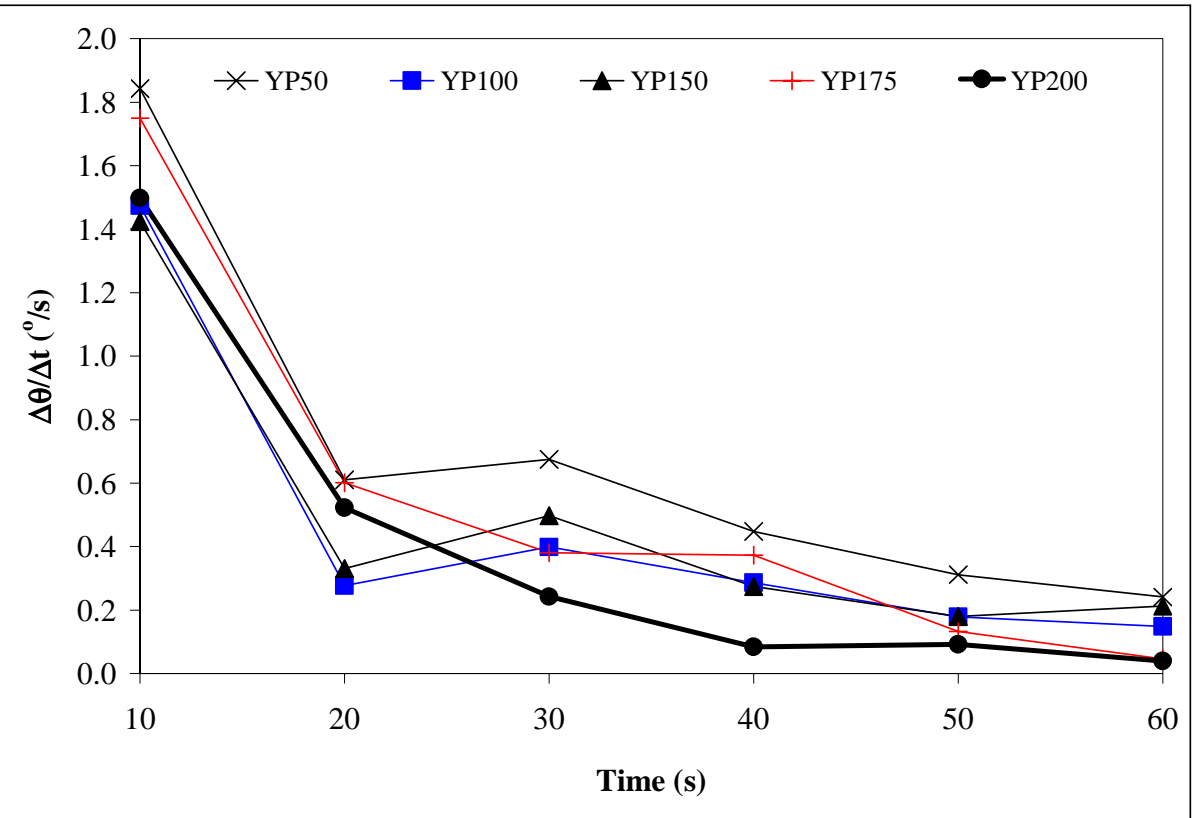

Figure 3.21. The rate of contact angle decline for yellow-poplar.

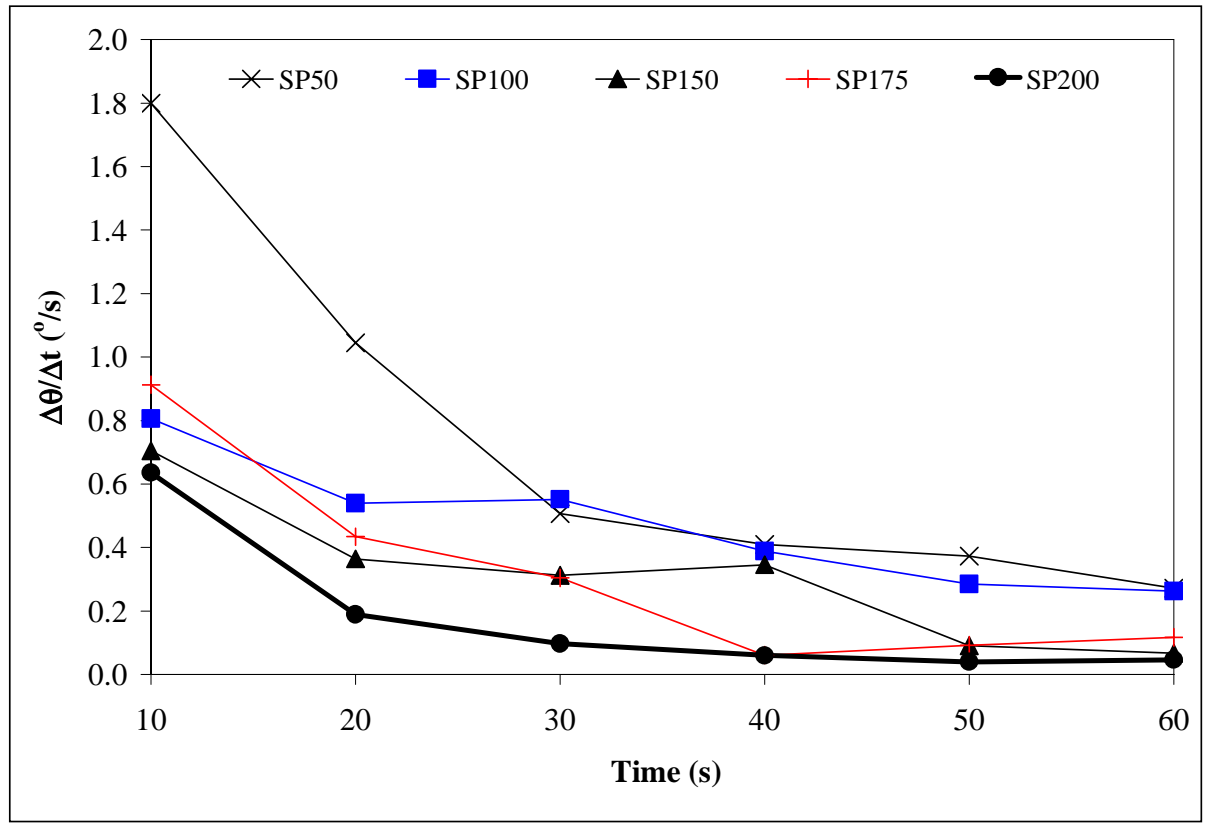

Figure 3.22. The rate of contact angle decline for southern pine. 


\subsubsection{Influence of Drying Temperature on Adhesive Bond Performance}

The corrected shear compliance method was used to estimate a maximum value $\left(\mathrm{G}_{\max }\right)$ and an arrested value $\left(\mathrm{G}_{\text {arr }}\right)$ of the strain energy release rate (SERR) of PF bonded specimens. The $G_{\max }$ refers to the maximum needed energy for crack initiation and crack growth in an adhesive bond; while $G_{\text {arr }}$ refers to energy associated with arrest of the crack. The maximum load, the arrested load, and the compliance were found for each cycle within a test specimen (Figure 3.23). The results of fracture test measurements and descriptive statistics are presented in Appendix D and Appendix E.

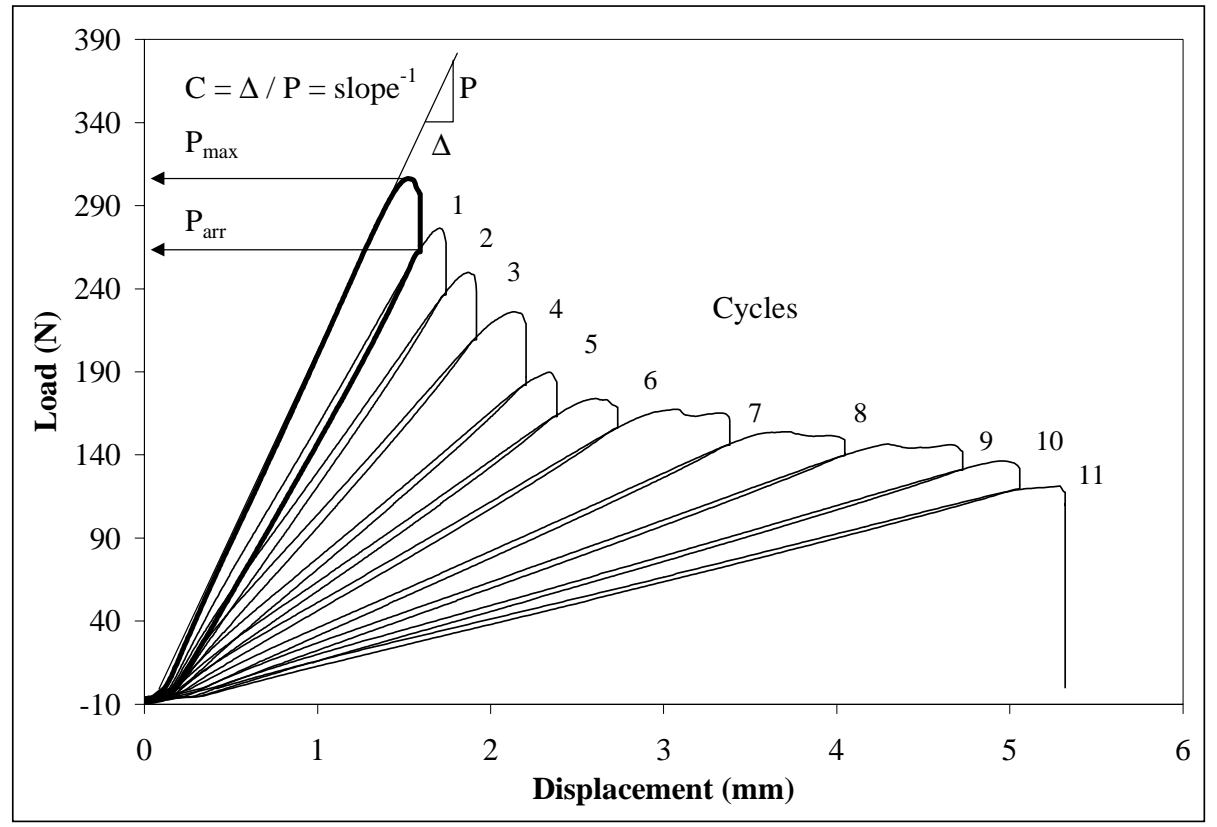

Figure 3.23. A typical load-displacement curve obtained from DCB by fracture testing.

These measurements were used to analyze data according to the procedure explained by Gagliano and Frazier (2001). A brief explanation of data analysis is given as follows. Plotting the cube root of compliance versus crack length (Figure 3.24), and fitting this data with a linear trendline, provided information needed for calculation of SERR $\left(\mathrm{G}_{\mathrm{I}}\right)$ by using equations 2.10, 2.11, and 2.12. Each cycle provided one data point for $G_{\max }$ and $G_{\text {arr }}$, but only data obtained along the specimen's length from 50 to $150 \mathrm{~mm}$ was used for the results (Figure 3.25). 


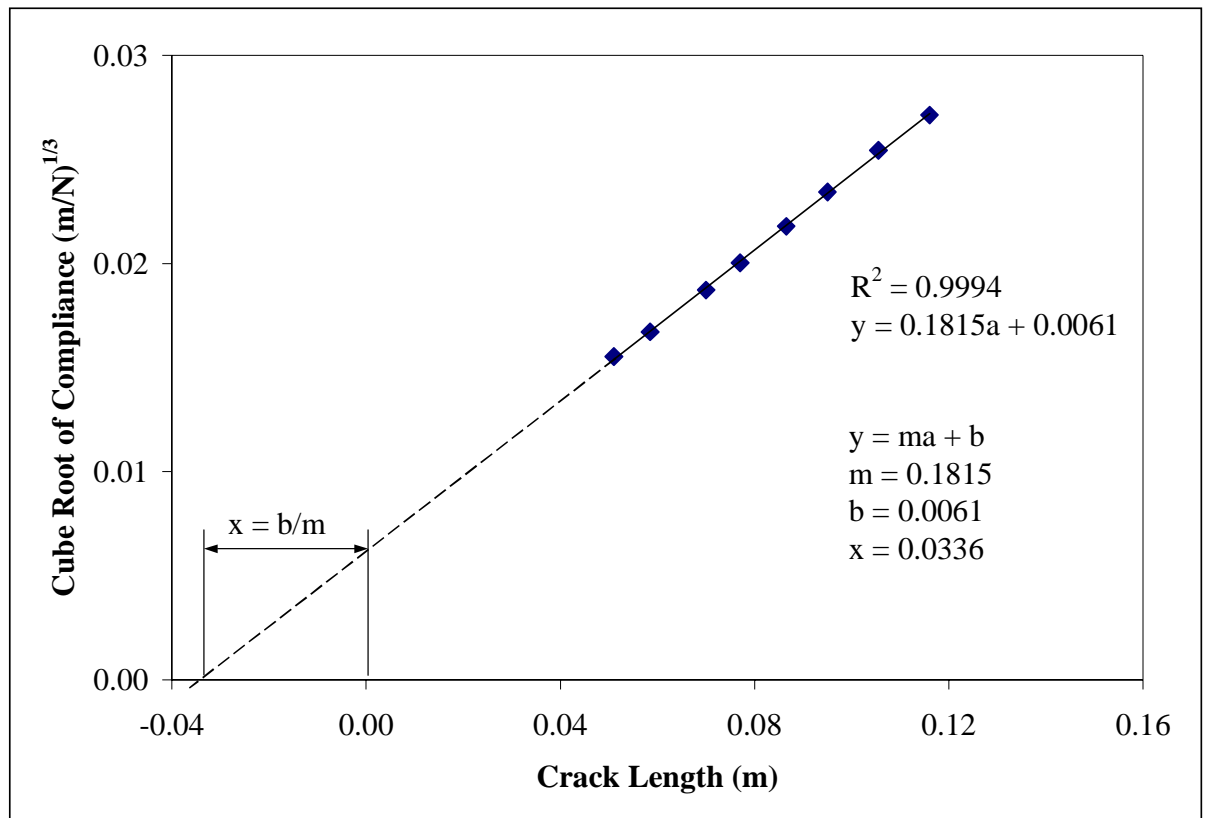

Figure 3.24. A typical plot of the cube root of compliance versus crack length.

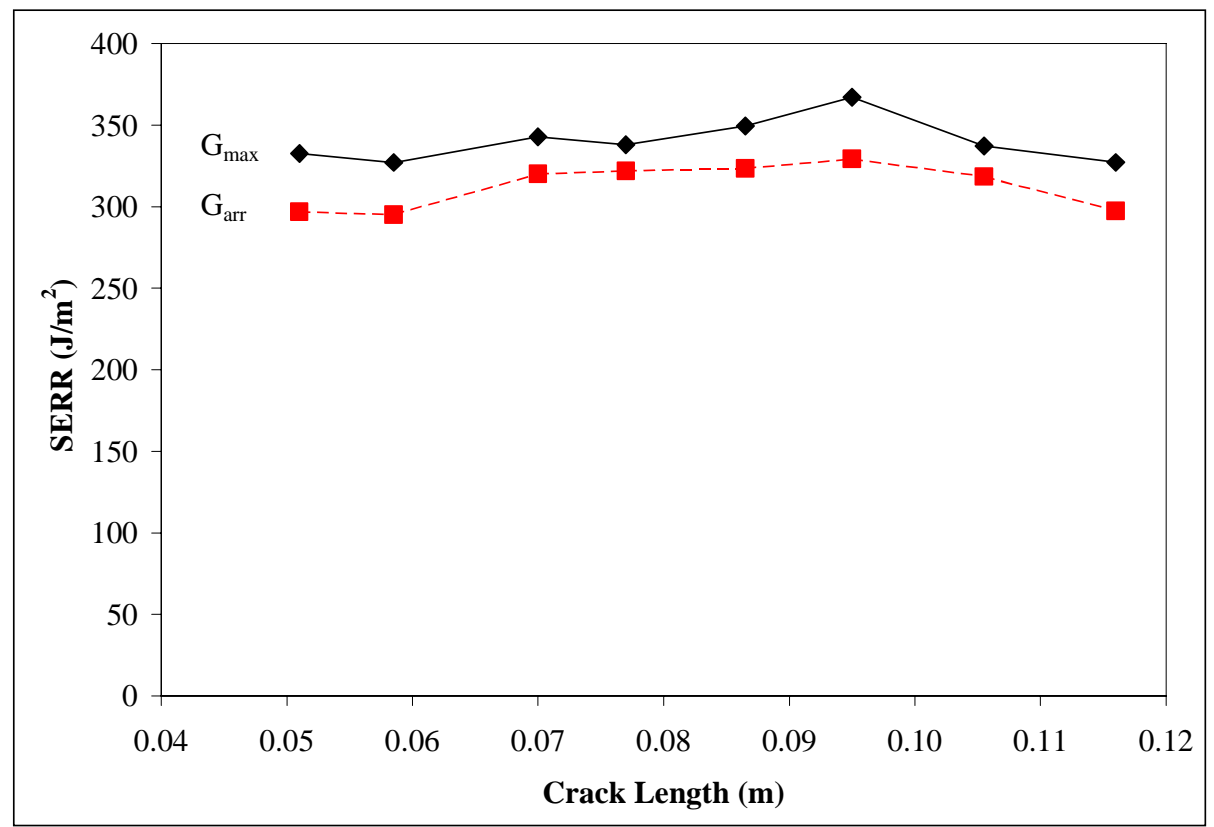

Figure 3.25. A typical plot of SERR versus crack length for a single DCB specimen. 
There was a slight variation in this data along the crack length because the wood surface is not homogeneous. The SERR should be material property of the system (Gagliano and Frazier 2001) and all data should be the same within a bondline. An average value of SERR was calculated for each specimen. The value of $G_{\max }$ was always higher than $G_{\text {arr }}$, but both had a similar dependence on crack length. The average values of maximum and the arrested SERR are shown in Table 3.5 and Table 3.6.

Table 3.5. Average strain energy release rate $\left(\mathrm{J} / \mathrm{m}^{2}\right)$ for yellow-poplar adhesive bond.

\begin{tabular}{|c|c|c|c|c|c|c|}
\hline \multirow[b]{2}{*}{ Adhesive } & \multirow{2}{*}{$\begin{array}{c}\text { Wood Species } \\
\text { YP } \\
\end{array}$} & \multicolumn{5}{|c|}{ Maximum Surface Temperature $\left({ }^{\circ} \mathrm{C}\right)$} \\
\hline & & 51 & 104 & 156 & 172 & 187 \\
\hline \multirow{2}{*}{$\mathbf{P F}$} & $\mathbf{G}_{\max }$ & 368.6 & 323.2 & 321.5 & 300.8 & 319.9 \\
\hline & $\mathbf{G}_{\text {arr }}$ & 321.9 & 276.8 & 285.4 & 264.0 & 283.2 \\
\hline \multirow{2}{*}{ PVA } & $\mathbf{G}_{\max }$ & 315.4 & 313.9 & 374.9 & 365.4 & 308.0 \\
\hline & $\mathbf{G}_{\text {arr }}$ & 285.6 & 280.4 & 328.5 & 305.3 & 287.9 \\
\hline
\end{tabular}

Table 3.6. Average strain energy release rate $\left(\mathrm{J} / \mathrm{m}^{2}\right)$ for southern pine adhesive bond.

\begin{tabular}{|c|c|c|c|c|c|c|}
\hline \multirow[b]{2}{*}{ Adhesive } & \multirow{2}{*}{$\begin{array}{c}\text { Wood Species } \\
\text { SP }\end{array}$} & \multicolumn{5}{|c|}{ Maximum Surface Temperature $\left({ }^{\circ} \mathrm{C}\right)$} \\
\hline & & 51 & 104 & 156 & 172 & 187 \\
\hline \multirow{2}{*}{ PF } & $\mathbf{G}_{\max }$ & 229.5 & 216.5 & 109.5 & 143.7 & 75.7 \\
\hline & $\mathbf{G}_{\text {arr }}$ & 192.3 & 179.5 & 96.6 & 116.0 & 60.7 \\
\hline \multirow{2}{*}{ PVA } & $\mathbf{G}_{\max }$ & 169.8 & 171.0 & 166.2 & 160.6 & 83.0 \\
\hline & $\mathbf{G}_{\text {arr }}$ & 135.2 & 124.9 & 134.1 & 128.4 & 55.2 \\
\hline
\end{tabular}

Both, the adhesive and the wood species affected the magnitude of $G_{\max }$ and $G_{\text {arr }}$. However, significant differences in $\mathrm{G}_{\max }$ among different temperature exposures were obtained only for SP samples. YP surfaces were much better substrates for bonding. These specimens exhibited a higher average value of $\mathrm{G}_{\max }$ than $\mathrm{SP}$ specimens regardless of the drying temperature or adhesive used. If $\mathrm{G}_{\max }$ is used to evaluate wood surface inactivation, one can conclude that even though there was a tendency of decreasing $G_{\max }$ for the PF adhesive bond, it is obvious that YP surfaces were not susceptible to inactivation due to high temperature exposure (Figure 3.26). On the other hand, SP surfaces that were exposed to drying temperature $>156^{\circ} \mathrm{C}$, exhibited high susceptibility to inactivation (Figure 3.27). 


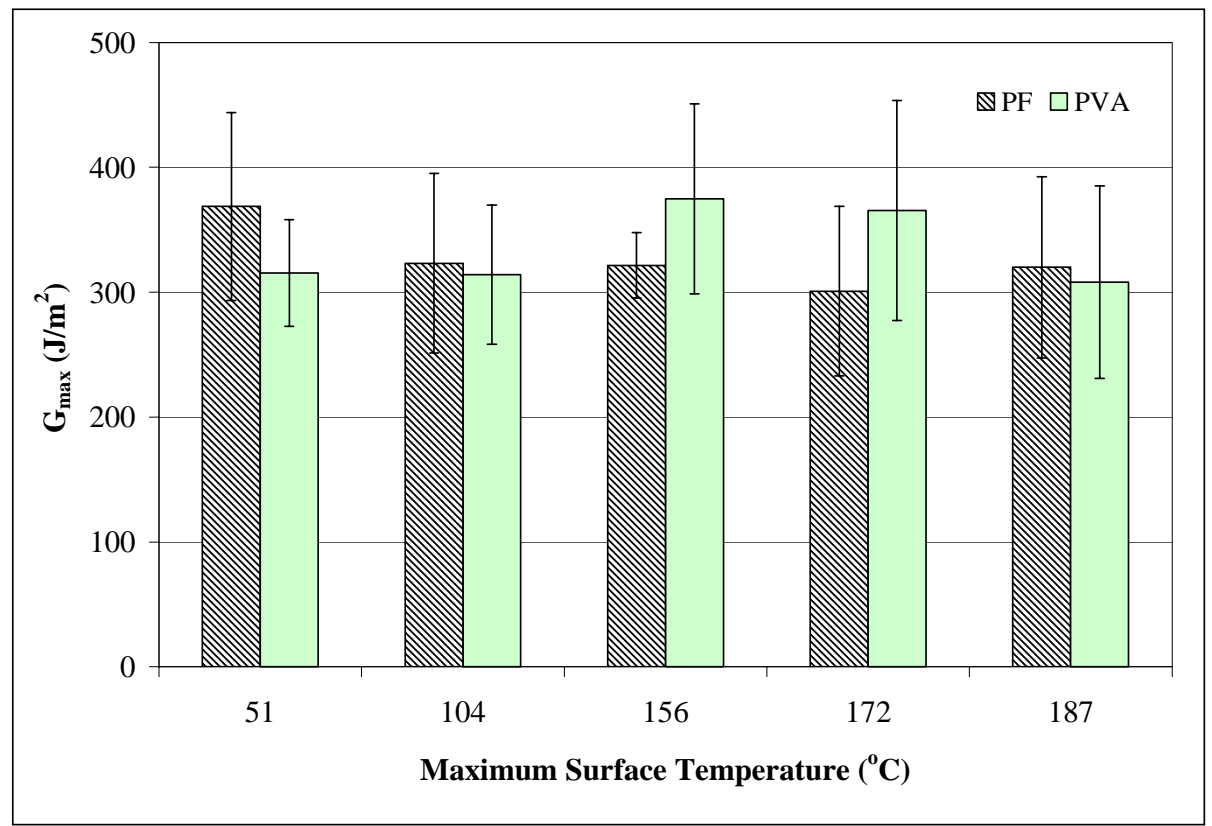

Figure 3.26. Influence of drying temperature on the maximum strain energy release rate of yellow-poplar adhesive bond.

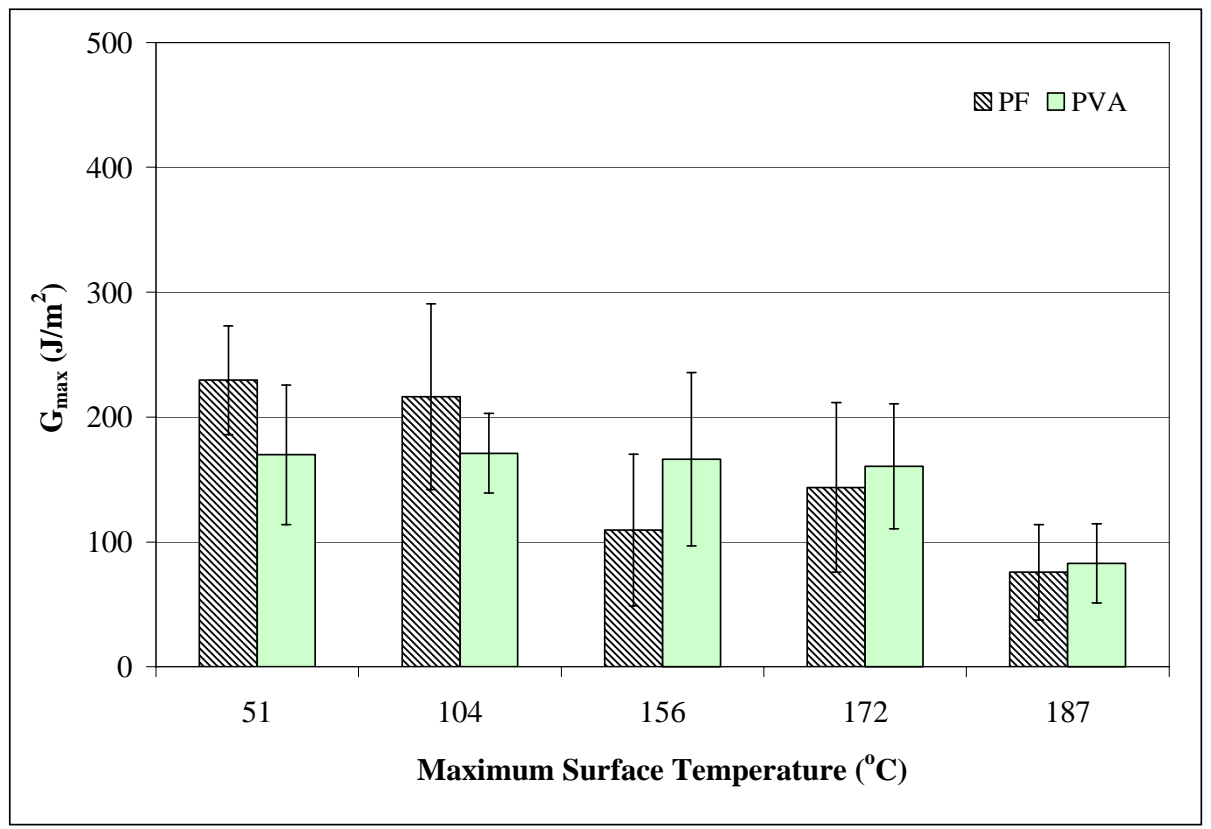

Figure 3.27. Influence of drying temperature on the maximum strain energy release rate of southern pine adhesive bond. 
In terms of $G_{\max }$, YP was not susceptible to inactivation at any drying temperature exposure up to $187^{\circ} \mathrm{C}$ while SP exhibited severe surface inactivation. When PF adhesive was used, $\mathrm{G}_{\max }$ dropped significantly for SP samples that were exposed to the temperature of $156^{\circ} \mathrm{C}$ or higher. When PVA adhesive was used, $\mathrm{G}_{\max }$ was almost constant for temperature exposures up to $172^{\circ} \mathrm{C}$, and then it dropped significantly at the temperature of $187^{\circ} \mathrm{C}$. Differences in flow characteristics, surface energy, cure kinetics, and polymer composition or structure may offer an explanation for variation of $\mathrm{G}_{\max }$ between PF and PVA. Moreover, it can be speculated that the cure of PF adhesives was retarded by increased acidity of SP surfaces. This possibility was reported in other studies (Subramanian 1984; Hse and Kuo 1988). Many of SP extractives are acid (e.g., resin acids and fatty acids). When extractives or VOCs components concentrate at the wood surfaces, its $\mathrm{pH}$ value decreases. A low $\mathrm{pH}$ inhibits the polymerization of alkaline type of PF adhesive (Pizzi 1983).

The evaluation of adhesion by using a DCB fracture specimen was an adequate procedure to indicate wood surface inactivation. Adhesion was low on inactivated wood surfaces and high on active wood surfaces. The broken adhesive bondline assembled from YP wood, which did not experience significant inactivation, showed many loose wood fibers imbedded in the adhesive. The drying temperature affected the location of the failure surface at the bond. For the YP specimens that experienced a surface temperature of $51^{\circ} \mathrm{C}$, cohesive wood failure dominated, but the crack propagation remained in the bondline (Figure 3.28, left). YP specimens that experienced surface temperature of $187^{\circ} \mathrm{C}$ exhibited no cohesive wood failure (Figure 3.28, right). However, as shown above (Figure 3.26), the PF adhesive bond performance of YP did not decrease significantly in regard to drying temperature. This was because in all cases of YP bonding, the adhesive wet the surface sufficiently, so that many secondary attractive forces were establish between the adhesive and the wood. 

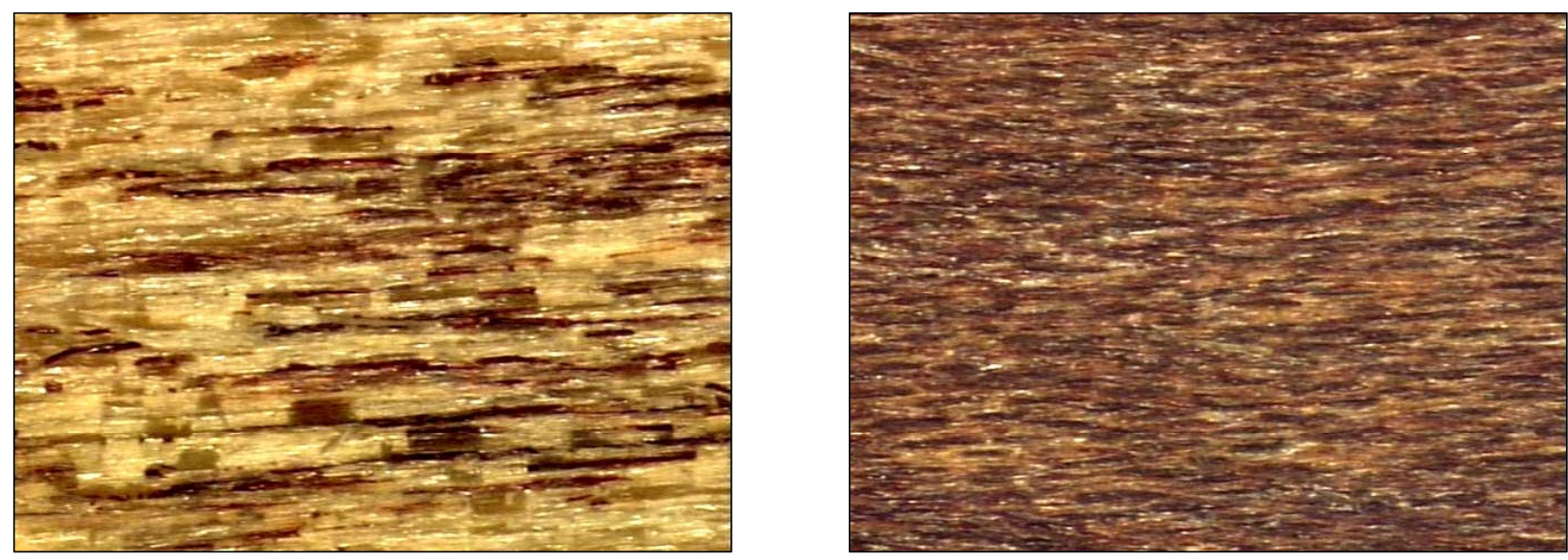

Figure 3.28. PF adhesive bond failure in regard to drying temperature exposure: YP dried at $51^{\circ} \mathrm{C}$ (left), and YP dried at $187^{\circ} \mathrm{C}$ (right).

Broken adhesive bondlines assembled from SP wood, which were dried at 51 and $104{ }^{\circ} \mathrm{C}$, showed some loose wood fibers imbedded in the adhesive (Figure 3.29, left). A completely different failure pattern was exhibited by SP surfaces, which were exposed to the drying temperature of $156^{\circ} \mathrm{C}$ and higher. In these cases, the adhesive bond failed without any wood failure (Figure 3.29, right), and the broken adhesive bondline showed the imprint of the opposite adherend.
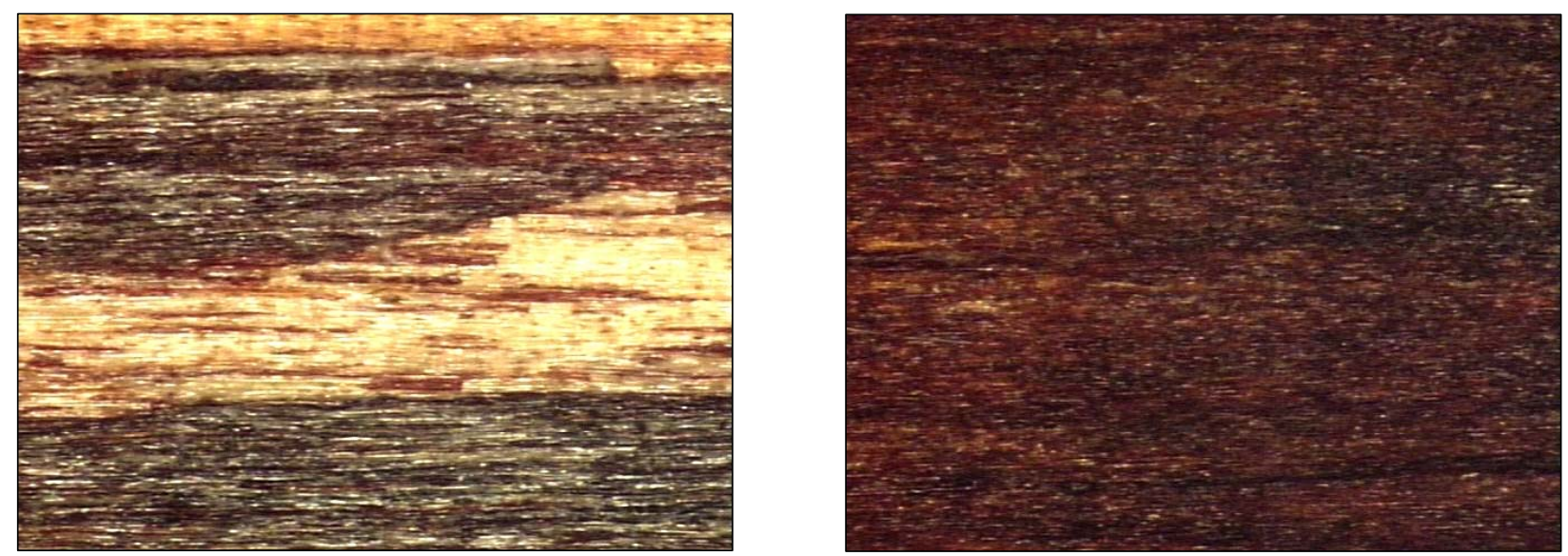

Figure 3.29. PF adhesive bond failure in regard to drying temperature exposure: SP dried at $51^{\circ} \mathrm{C}$ (left), and SP dried at $187^{\circ} \mathrm{C}$ (right). 
Severely inactivated wood surfaces (e.g., $\Delta \theta / \Delta \mathrm{t}<20^{\circ} / \mathrm{min}$ or $\theta_{\mathrm{i}}>90^{\circ}$ ) exhibited the weakest adhesion $\left(\mathrm{G}_{\max }\right)$. The adhesive bondline, in some cases, may have been undercured (Figure 3.30, left), when inactivation was extremely severe. Hancock (1963) and Wellons (1980) observed the similar behavior. Undercured adhesive refers to the solidification of adhesive, which is interrupted or terminated before being fully accomplished (Marra 1992). Undercuring yields a bond with low strength and reduced durability.

A poor adhesive bond can be due to enormous concentration of resinous extractives at the SP surfaces. Resinous extractives were seen to concentrate extensively on the SP surface (Figure 3.30, right). This indicated that adhesive could not make an intimate contact with the wood substrate, which was a result of poor wettability, as described in the previous section. A low adhesion of inactivated wood surfaces was probably associated with hydrophobic extractives, which were concentrated on the surface. Non-polar extractives of SP could be strongly enriched in the outer layer of wood during kiln (oven) drying (Zavarin 1984). In addition, these acid extractives may have impeded a curing reaction of $\mathrm{PF}$ adhesive ( $\mathrm{pH}$ of 10.5) that should proceed under alkali conditions.
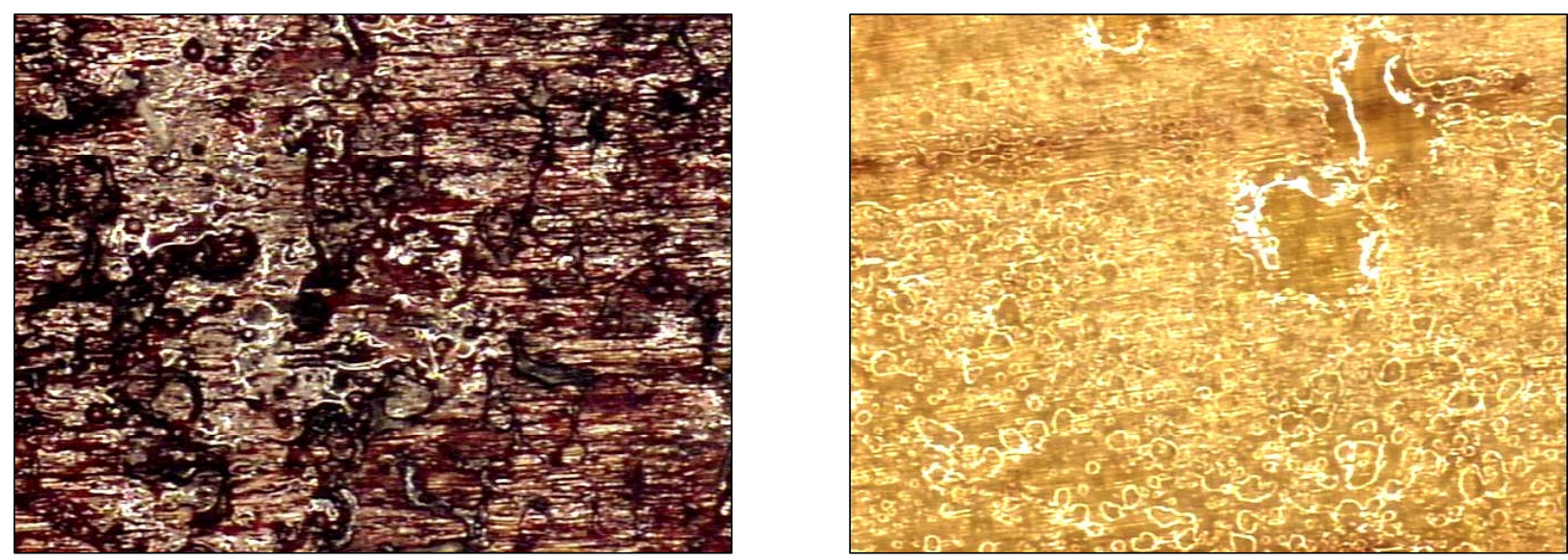

Figure 3.30. A poor adhesive bond (left) caused by extensive deposition of extractives on the SP surface (right). 
On the other hand, PVA adhesive was acid ( $\mathrm{pH}$ of 3.3) and pre-polymerized. Therefore, acid extractives on the wood surface did not affect solidification of PVA adhesive, as they did when PF was used. However, water-borne PVA probably could not reach and attach to the wood substrate either because surface was covered with HC-type, non-polar extractives. Wood extractives contribute practically no strength to wood. Therefore, if some partially connections between them and PVA adhesive occurred anyway, these did not significantly contribute to the adhesive bond performance. Thus, the PVA adhesive bond with SP failed at a small load.

\subsubsection{Adhesive Penetration}

Deposition of extractives at the surfaces and their degradation can effect adhesive penetration into wood (Yoshimoto 1989). In order to find a possible relationship between inactivated wood surface and adhesive penetration, EP and MP of PF adhesive were evaluated. When compared within the same wood species, the results showed that surface inactivation did not significantly affect PF adhesive penetration (Table 3.7) and Appendix F.

Table 3.7. Phenol-formaldehyde adhesive penetration into wood.

\begin{tabular}{|c|c|c|c|c|}
\hline \multirow{3}{*}{$\begin{array}{c}\text { Maximum Surface } \\
\text { Temperature }\left({ }^{\circ} \mathrm{C}\right)\end{array}$} & \multicolumn{4}{|c|}{ Adhesive Penetration ( $\mu \mathrm{m})$} \\
\hline & \multicolumn{2}{|c|}{ Yellow-Poplar } & \multicolumn{2}{|c|}{ Southern Pine } \\
\hline & 51 & 187 & 51 & 187 \\
\hline EP & 19.9 & 22.4 & 15.5 & 16.3 \\
\hline MP & 316.9 & 270.0 & 157.5 & 125.2 \\
\hline
\end{tabular}

When compared between wood species, the Duncan multiple range test (95\% confidence level) indicated that PF adhesive penetrated significantly better into YP wood than into SP wood. The differences are attributed to the anatomy of these species. YP is a hardwood, which contains vessels with perforate openings and large pits, through which PF adhesive could easily penetrate (Figure 3.31). On the other hand, SP is a softwood comprised mainly of longitudinal tracheids, which are less permeable for adhesives (Figure 3.32). Also, aspiration of pits could occur in SP, when the wood was dried at elevated temperatures. During drying, high capillary forces are established and the pit membrane is forced to move into the aspirated position where the tori 
remains hydrogen-bonded to the surface of the pit border (Siau 1995). This closes off a pit aperture resulting in reduced permeability, which could diminish adhesive penetration in SP.
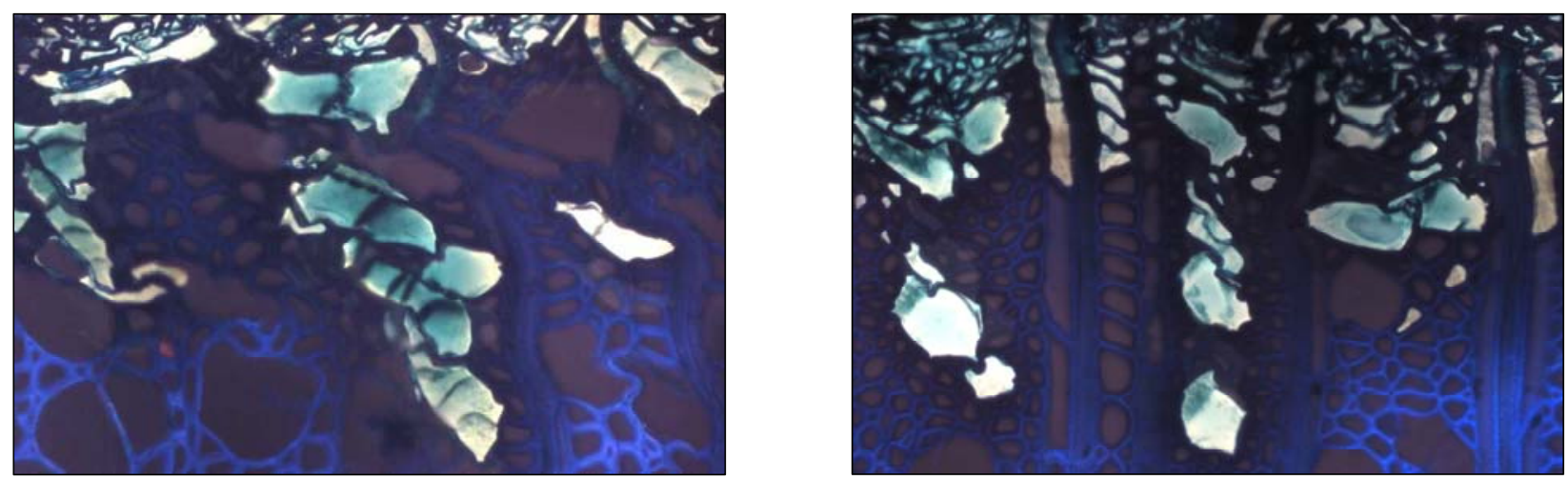

Figure 3.31. PF adhesive penetration into YP exposed to $51^{\circ} \mathrm{C}$ (left) and $187^{\circ} \mathrm{C}$ (right).
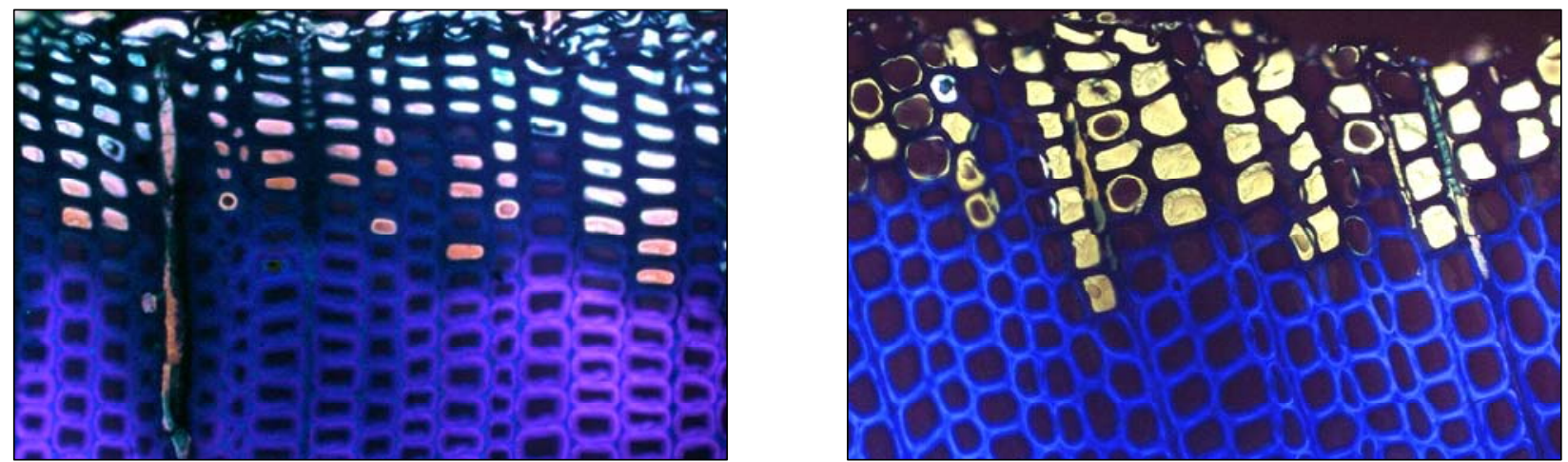

Figure 3.32. PF adhesive penetration into SP exposed to $51^{\circ} \mathrm{C}$ (left) and $187^{\circ} \mathrm{C}$ (right).

Within the same wood species, a significant difference in adhesive penetration in regard to drying temperature was not indicated. However, EP and MP did not provide any information about adhesive penetration into wood cell walls, which is critical for adhesion. Thus, it might happen that adhesive penetrated through lumens and pits of inactivated wood, but it did not interact with lignocellulosic substances, since cell walls at the surface were covered with thin layer of hydrophobic extractives. 


\subsubsection{Relationships among Wood Surface Chemistry, Wettability, and Adhesion}

\subsubsection{Wettability and Chemical Composition}

An initial wettability index (i.e., $\cos \theta_{\mathrm{i}}$ ) was plotted against the $\mathrm{O} / \mathrm{C}$ ratio, and also against the $\mathrm{C} 1 / \mathrm{C} 2$ ratio in order to find a possible relationship between the wetting capacity of the wood surface and its chemical composition. The cosine function was selected because of the relationship among interfacial surface tension of vapor (V), liquid (L) and solid (S) phases. In fact, $\cos \theta$ is often used as a direct measure of surface wettability (Kajita and Skaar 1992). A strong linear relationship was found between wettability and surface chemistry. When $\cos \theta_{\mathrm{i}}$ was plotted against the $\mathrm{O} / \mathrm{C}$ and the $\mathrm{C} 1 / \mathrm{C} 2$ ratio, a linear statistical model explained most of the variability -87 and $90 \%$, respectively. Wettability of the wood surface increased with the O/C ratio (Figure 3.33) and it decreased with the $\mathrm{C} 1 / \mathrm{C} 2$ ratio (Figure 3.34).

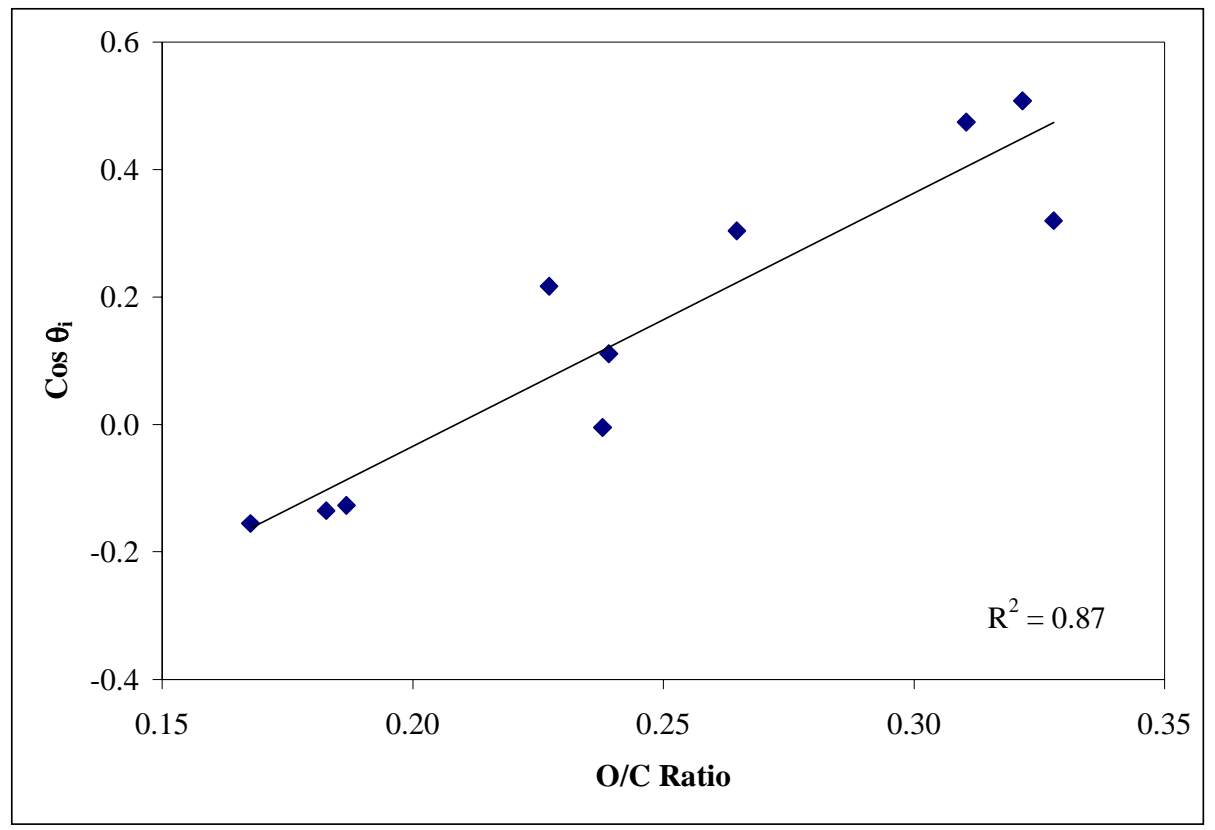

Figure 3.33. Relationship between initial wettability of $Y P$ and $S P$ and the $O / C$ ratio. 


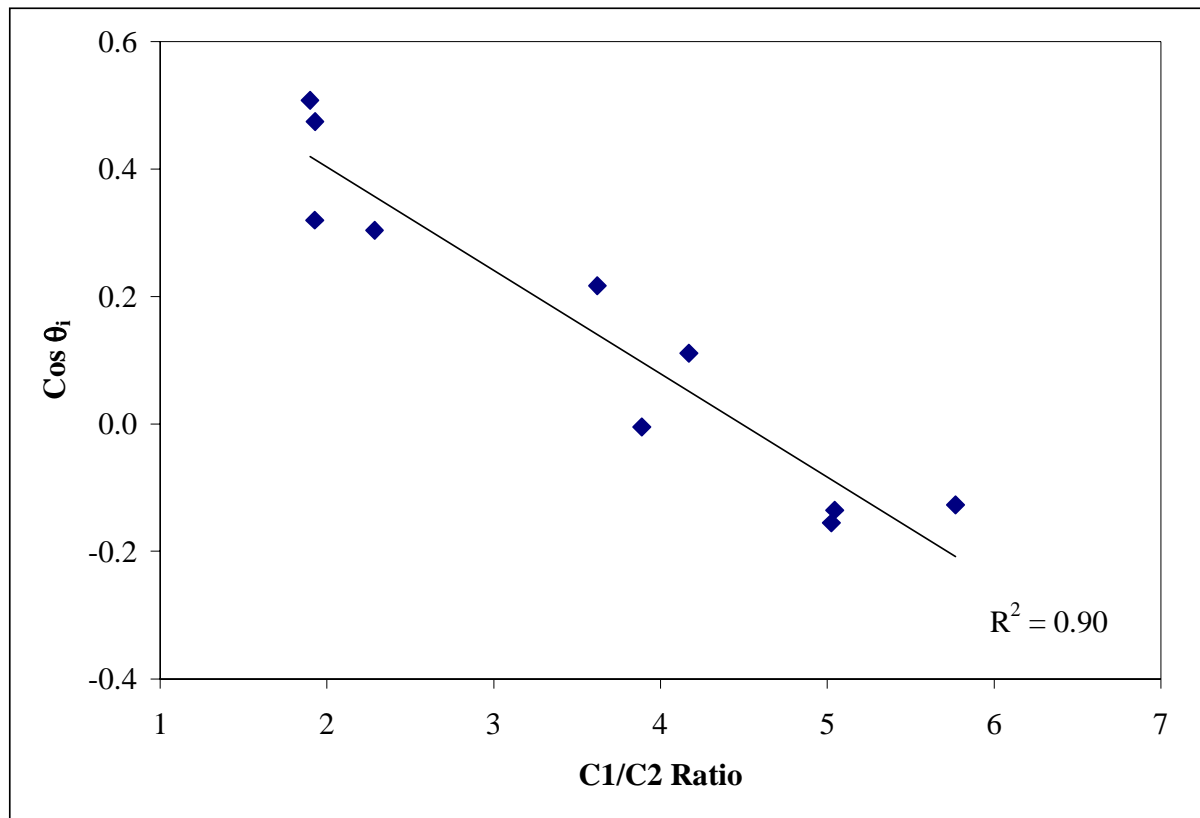

Figure 3.34. Relationship between initial wettability of YP and SP and the C1/C2 ratio.

The wettability index was low on wood surfaces that had a low $\mathrm{O} / \mathrm{C}$ ratio or a high $\mathrm{C} 1 / \mathrm{C} 2$ ratio. These ratios represented a hydrophobic surface. When the $\mathrm{O} / \mathrm{C}$ ratio increased or the $\mathrm{C} 1 / \mathrm{C} 2$ ratio decreased, the concentration of hydrophobic material decreased on the surface, thus wettability was improved.

\subsubsection{Wettability and Adhesion}

Wettability is crucial for good adhesion in wood bonding. Adhesive has to wet, flow, and penetrate the cellular structure of wood in order to establish intimate contact between molecules of wood and adhesive (USDA 1999). The results obtained support the fact that wettability plays an important role when bonding wood surfaces with water-borne adhesives. The highest values of the $\mathrm{G}_{\max }$ were obtained at high $\cos \theta$, (i.e., low contact angle), which presents good wettability. $\mathrm{G}_{\max }$ increased with $\cos \theta$, regardless of wood species. A linear statistical model explained $90 \%$ of the variability for PF bonded samples, and 75\% for PVA bonded samples. Adhesive bond performance increased with increasing water wettability for both adhesives and regardless of wood species (Figure 3.35 and Figure 3.36). 


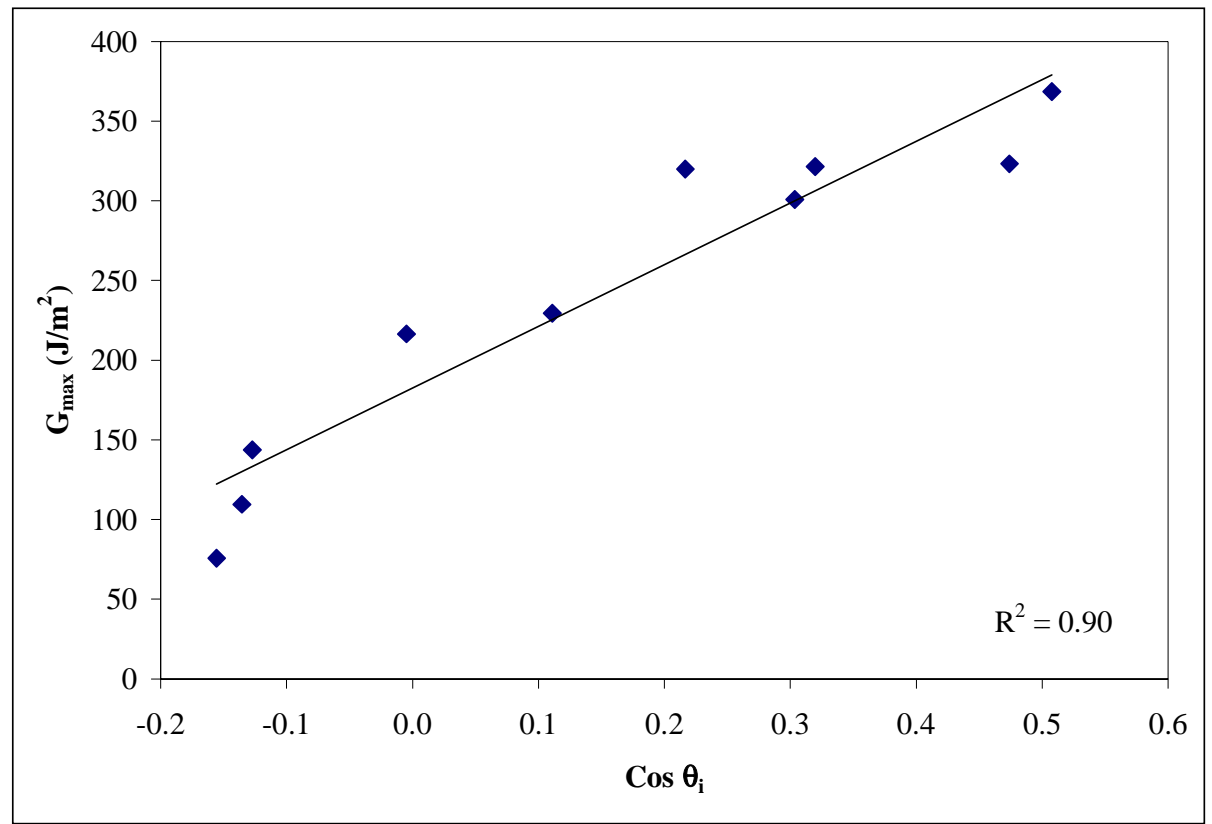

Figure 3.35. Relationship between adhesion and wettability for YP and SP bonded with PF.

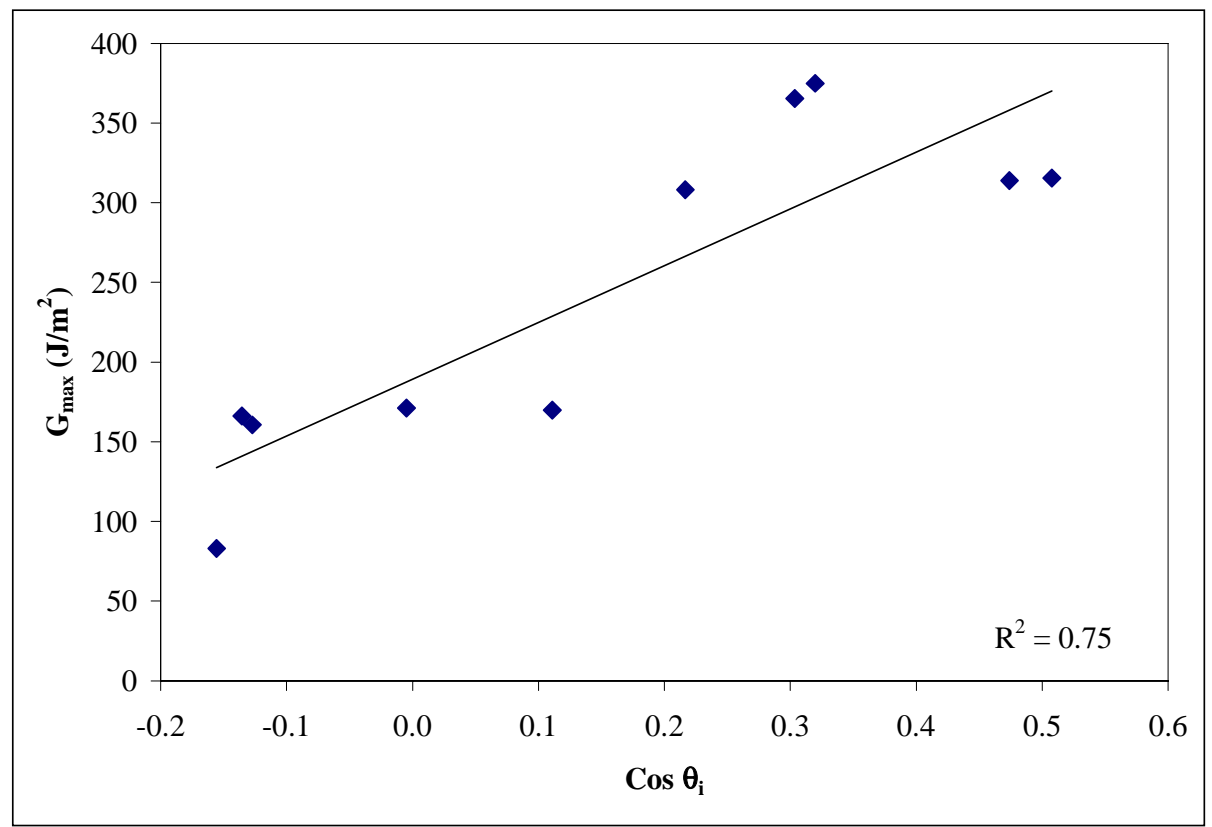

Figure 3.36. Relationship between adhesion and wettability for YP and SP bonded with PVA. 
$\mathrm{G}_{\max }$ of PF adhesive bond was also plotted against the rate of contact angle change. A strong linear relationship was obtained for SP samples that were bonded with PF adhesive (Figure 3.37) and a less pronounced relationship was obtained for YP samples that were bonded with PF adhesive (Figure 3.38). Samples bonded with PVA adhesive did not exhibit a clear relationship between $\mathrm{G}_{\max }$ and $\Delta \theta / \Delta \mathrm{t}$.

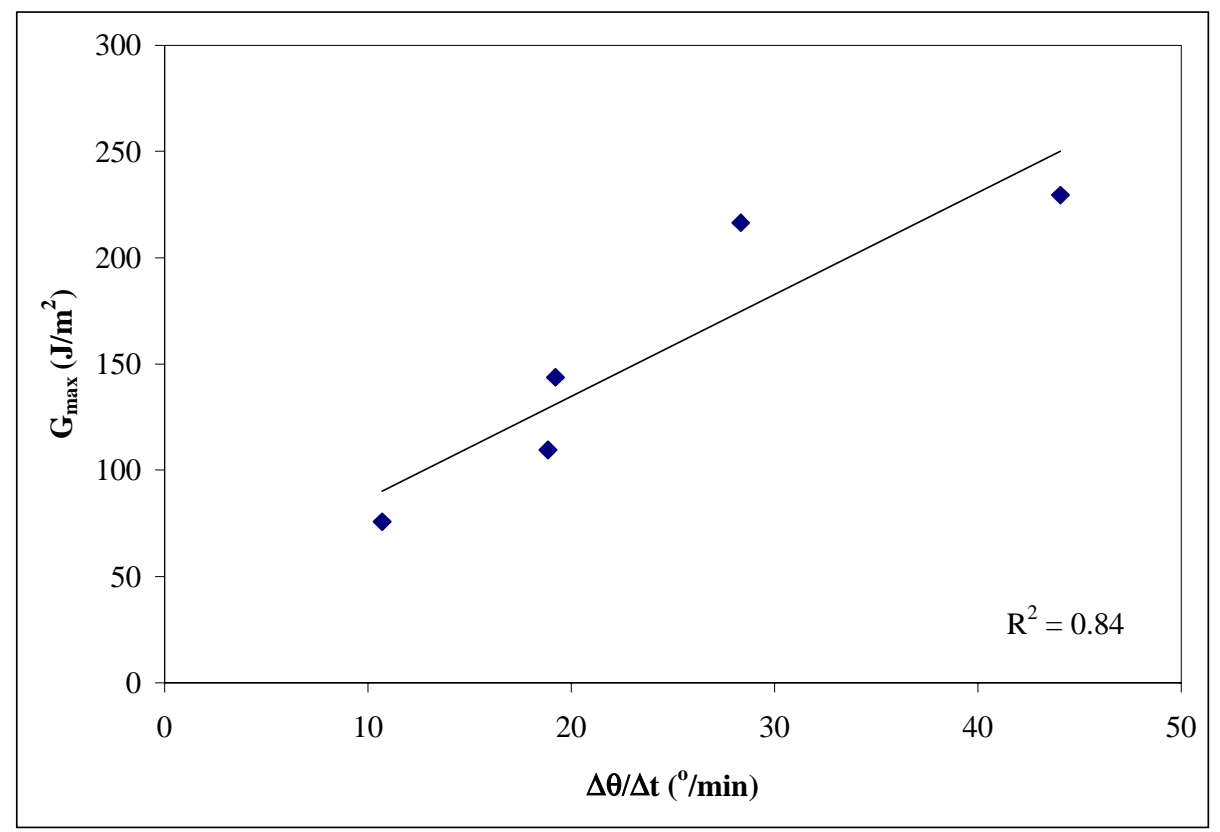

Figure 3.37. Relationship between adhesion and rate of contact angle change for SP samples bonded with PF adhesive. 


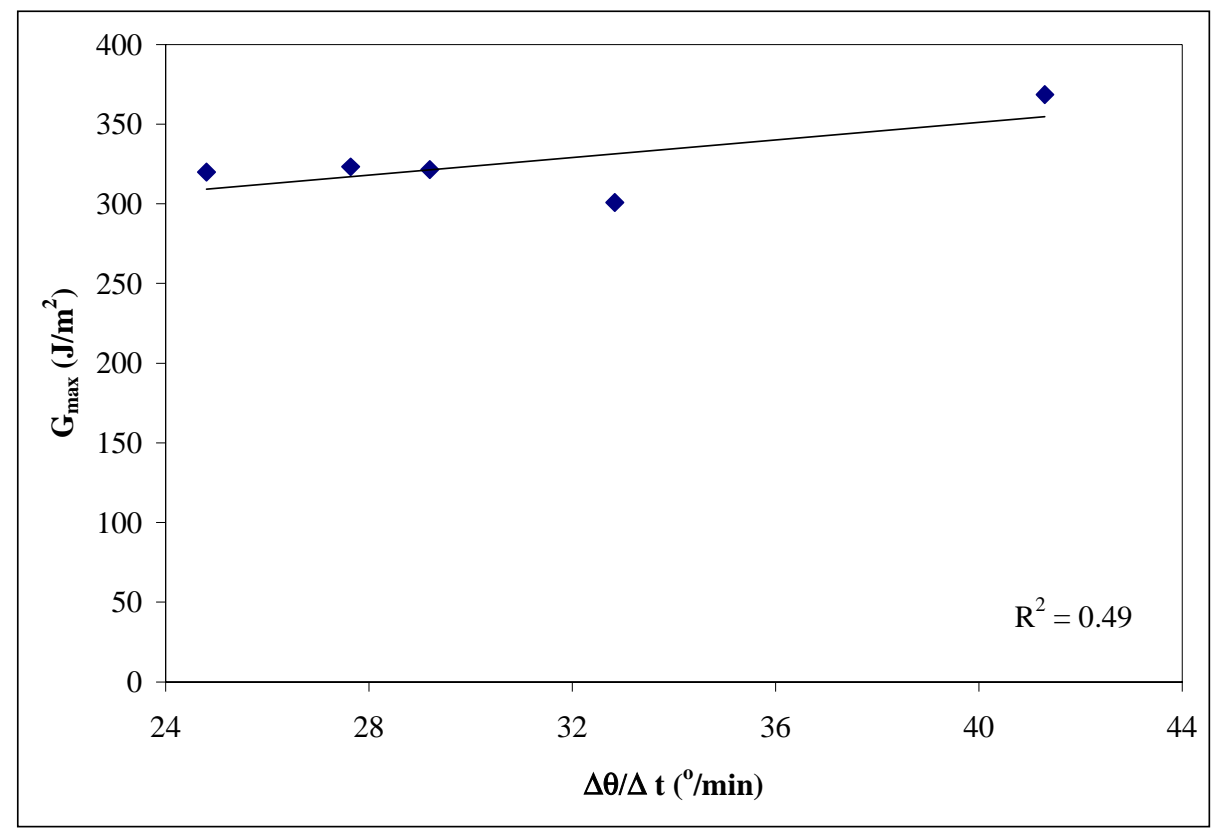

Figure 3.38. Relationship between adhesion and rate of contact angle change for YP samples bonded with PF adhesive.

\subsubsection{Chemical Composition and Adhesion}

A linear relationship was found between $\mathrm{G}_{\max }$ and $\mathrm{O} / \mathrm{C}$ or $\mathrm{C} 1 / \mathrm{C} 2$ ratio. When $\mathrm{G}_{\max }$ was plotted against the $\mathrm{O} / \mathrm{C}$ ratio, a linear statistical model explained $81 \%$ of the variability for $\mathrm{PF}$ bonded samples, and $78 \%$ for PVA bonded samples (Figure 3.39). When $\mathrm{G}_{\max }$ was plotted against the $\mathrm{C} 1 / \mathrm{C} 2$ ratio, a linear statistical model explained $80 \%$ of the variability for PF bonded samples, and 69\% for PVA bonded samples (Figure 3.40). PF adhesives exhibited a more significant relationship between adhesive bond performance and surface chemistry than PVA adhesive. However, for both PF and PVA adhesives, $\mathrm{G}_{\max }$ increased with the $\mathrm{O} / \mathrm{C}$ ratio and decreased with the $\mathrm{C} 1 / \mathrm{C} 2$ ratio (Figure 3.39 and Figure 3.40). 


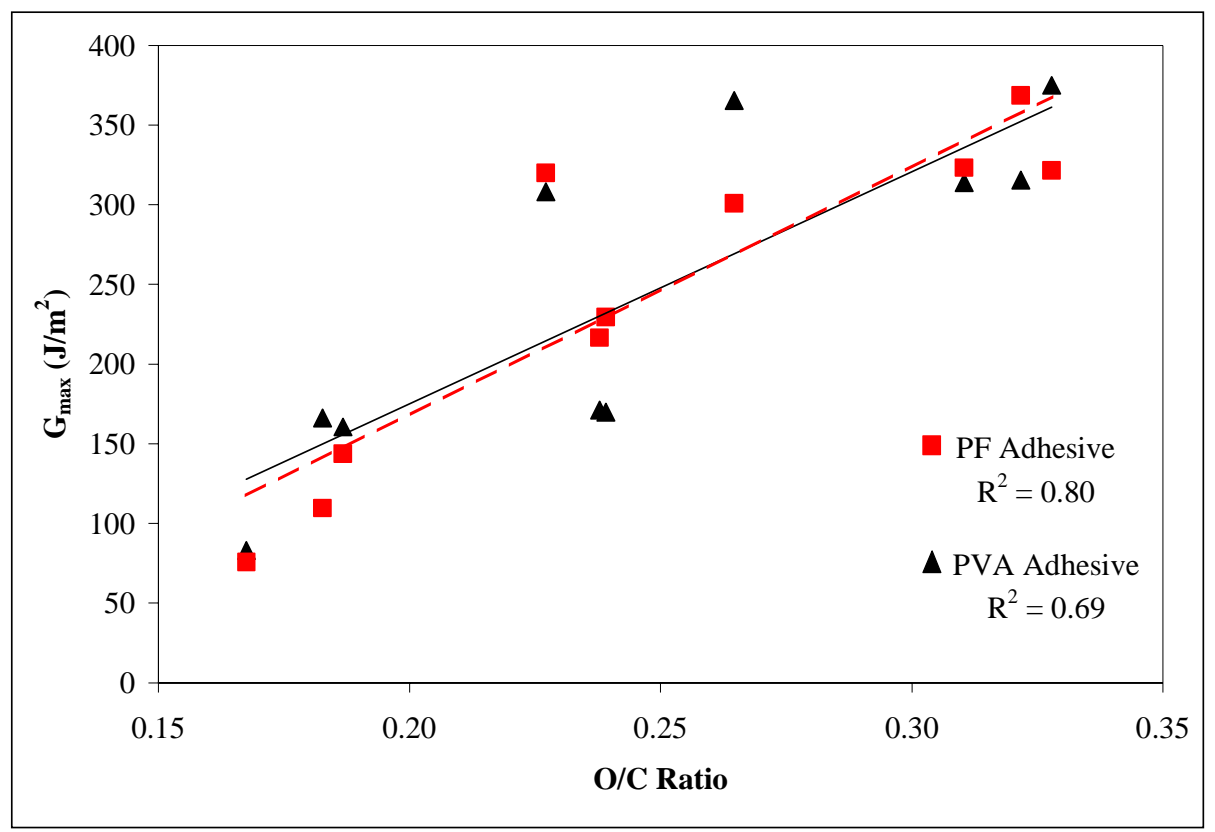

Figure 3.39. Relationship between adhesion and O/C ratio for YP and SP.

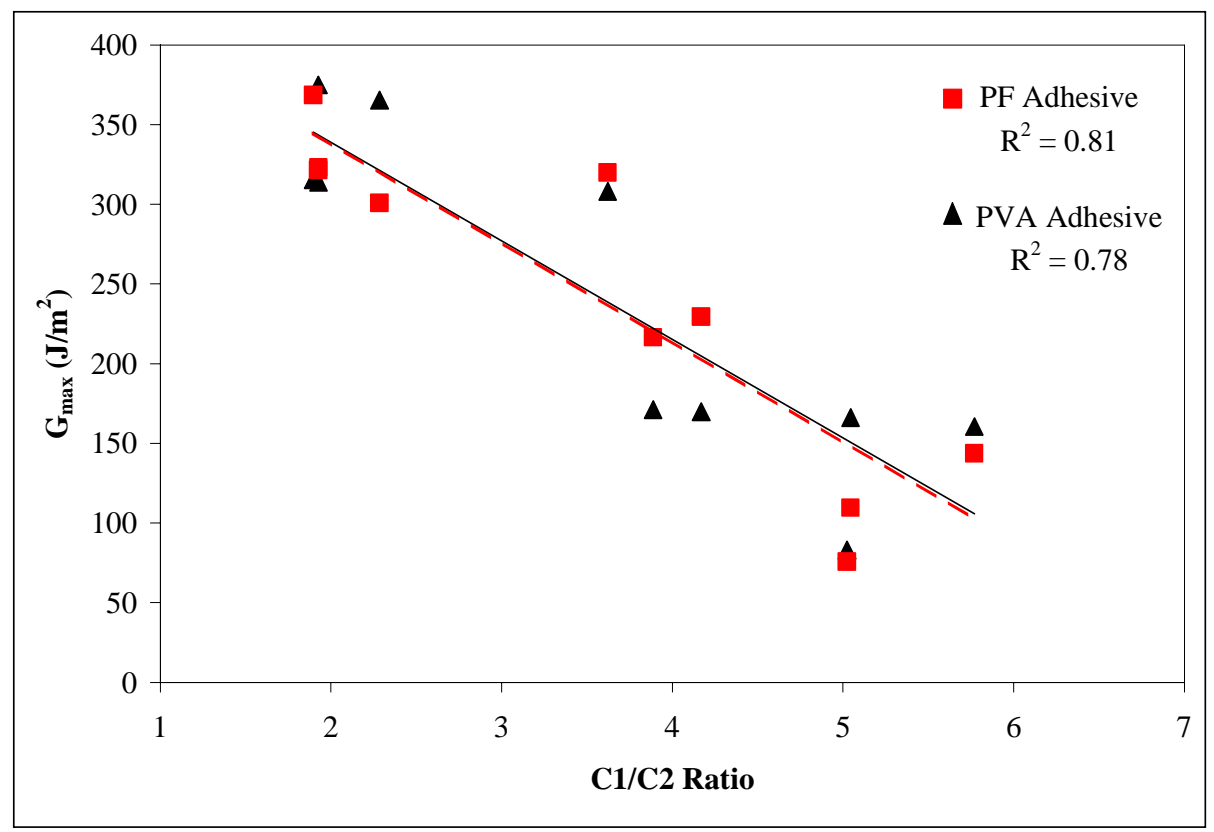

Figure 3.40. Relationship between adhesion and C1/C2 ratio for YP and SP. 
This relationship was expected since wood wettability increased with a decreased amount of extractives and lignin on the surface, as determined by the $\mathrm{O} / \mathrm{C}$ and $\mathrm{C} 1 / \mathrm{C} 2$ ratios. Wood wettability was promoted by increasing the $\mathrm{O} / \mathrm{C}$ ratio or by decreasing the $\mathrm{C} 1 / \mathrm{C} 2$ ratio, because extractives and lignin concentration on the surface decreased. Thus, the hydrophobic character of the surface decreased and also, the wood surface contained relatively more cellulose and hemicelluloses, which was probably also responsible for the improvement of the adhesive bond performance. Wood cellulose and hemicelluloses provide numerous bonding sites for adhesive through hydroxyl groups. Theoretically, there are three $\mathrm{OH}$-groups per each glucose unit (Fengel and Wegener 1989). These OH-groups are able to interact with functional groups of an adhesive by forming the hydrogen bond (H-bond). H-bonds are weaker (around $20 \mathrm{~kJ} / \mathrm{mole}$ ) by an order of magnitude in comparison with covalent bonds (Schrader and Loeb 1992), but hydrogen bonding can provide relatively strong secondary interaction when many hydrogen bonds are established. 


\subsection{Conclusions}

The strain energy release rate obtained by the fracture test showed that southern pine was more susceptible to surface inactivation than yellow-poplar. Adhesive bond performance of southern pine dropped by a factor of two for samples exposed to high temperature. From a mechanical standpoint, the southern pine surface was inactive for PF adhesive when dried at $156^{\circ} \mathrm{C}$ or higher, and for PVA adhesives when dried at $187^{\circ} \mathrm{C}$. Yellow-poplar surfaces did not show a significant inactivation phenomenon when exposed to drying temperatures up to $187^{\circ} \mathrm{C}$. Yellow-poplar surfaces exhibited higher adhesive bond performance than southern pine specimens regardless of the drying temperature or adhesive used.

Wood surface chemistry changed in regard to the drying temperature exposure. The oxygen to carbon ratio $(\mathrm{O} / \mathrm{C})$ decreased, and the $\mathrm{C} 1 / \mathrm{C} 2$ ratio increased with temperature. A low $\mathrm{O} / \mathrm{C}$ ratio and high $\mathrm{C} 1 / \mathrm{C} 2$ ratio reflected a high concentration of extractives and lignin on the wood surface. Both yellow-poplar and southern pine surfaces indicated higher extractives and lignin content for samples exposed to higher temperatures, which modified the wood surface from hydrophilic to hydrophobic. At temperate higher than $150^{\circ} \mathrm{C}$, emission and degradation of VOCs also occurred. A part of VOCs, which remained or was absorbed at the wood surface, could significantly alter the changes in surface chemistry and wettability. VOCs of southern pine are composed of hydrocarbons, which are non-polar and hydrophobic. Since the hydrophobic wood surface repelled water, wettability of this surface was low (i.e., a high contact angle). The highest contact angle was obtained on the surfaces, which were exposed to the drying temperature of $187^{\circ} \mathrm{C}$. The contact angle increased with drying temperature and decreased with time. Wood species affected wettability—southern pine exhibited higher contact angles than yellow-poplar at all studied temperature exposures.

The comparative analysis was able to elucidate clear relationships between surface chemistry, wettability, and bond performance in regard to surface inactivation. Extractive migration obviously plays a significant role in heat-induced surface inactivation. The higher the drying temperature the faster the extractives migration. Consequently, the higher the inactivation, the lower the wettability, and the weaker the adhesion. 


\section{Chapter 4. Wood Surface Chemistry, Wettability, and Adhesion}

\subsection{Introduction}

Wood cell walls contain three principal polymers: cellulose, hemicellulose, and lignin. A small amount of starch and proteins exist in wood as minor polymeric substances. Wood cellulose, which can be highly crystalline, serves as a framework; hemicelluloses, which are seldom crystalline, act as a matrix for the cellulose; and lignin, which is an amorphous substance of wood, surrounds and holds them together. Therefore, cellulose imparts strength to wood, lignin provides it stiffness, and hemicellulose gives it toughness (Winandy and Rowell 1984).

Cellulose is the main wood constituent, making up approximately half of both softwoods and hardwoods. Cellulose is a linear polyglucan with a uniform chain structure (Fengel and Wegener 1989). The cellulose chain is elongated and the glucose units are arranged in one plane. Hemicelluloses are chemically related to cellulose, since both are carbohydrates and polysaccharides built up from sugars (Sjöström 1993). Hemicelluloses differ from cellulose by their composition of various sugar units, a much shorter molecular chain, and a branching of the chain molecules. The main sugars in hemicelluloses are the hexoses (e.g., glucose, mannose, galactose) and the pentoses (e.g., xylose and arabinose). Lignin is a polymer composed of phenylpropane units. Softwoods usually contain more lignin than hardwoods. The lignin structure differs between softwood and hardwood species (Fengel and Wegener 1989).

Besides the cell wall components, there are numerous compounds, which are called the accessory or extractive materials of wood. They are low-molecular-weight substances and can be divided into organic and inorganic matter. The inorganic matter is ash. The organic matter is commonly called extractives. They are not part of the wood substance, but are deposited in cell lumen and cell walls. Extractives contents vary within trees, and are highly concentrated in certain parts of the tree (e.g., bark, heartwood, roots, areas of wound irritation). Extractives can be removed from wood by means of polar and non-polar solvents. Even though extractable components contribute only a few percent to the wood mass, they may greatly affect the physical and mechanical properties and processing of wood (Fengel and Wegener 1989). 


\subsubsection{Composition of Wood Surface}

The wood surface contains the same components as bulk wood, but the proportions among polysaccharides, lignin, and extractives may differ substantially. These differences in chemical composition of the wood surface are a function of the conditions and methods of surface formation (Zavarin 1984). The changes can occur during and after surface preparation.

Surface analytical methods differ from methods for bulk analysis because the object of observation is quite different. Spectroscopic methods such as X-ray photoelectron spectroscopy (XPS), Auger electron spectroscopy (AES), and secondary ion mass spectrometry (SIMS) are presently the most common routine methods for chemical surface characterization (Brune et al. 1997). Electron spectroscopy for chemical analysis (ESCA), an acronym for XPS, is widely used for analysis of polymers because it provides valuable data on atomic and chemical surface composition (Schrader and Loeb 1992). XPS spectroscopy can be used to analyze a wood surface. The results of XPS outline atomic percents, oxygen to carbon $(\mathrm{O} / \mathrm{C})$ atomic ratio, and $\mathrm{C} 1 / \mathrm{C} 2$ atomic ratio. The $\mathrm{C} 1$ type of the carbon atom presents the $\mathrm{C}-\mathrm{C}$ or $\mathrm{C}-\mathrm{H}$ bonds, while the $\mathrm{C} 2$ type of carbon refers to the $\mathrm{C}-\mathrm{O}$ bond. The atomic percent is not equal to the weight percent, which describes the chemical composition of the bulk wood. Atomic percent is the relative concentration of an element. The $\mathrm{O} / \mathrm{C}$ ratio and the $\mathrm{C} 1 / \mathrm{C} 2$ ratio are related to the chemical composition of wood constituents, which allows for the identification of the principal components of the wood.

Several studies confirmed the assignment of the $\mathrm{C} 1 \mathrm{~s}$ peak for lignocellulosic materials (Ahmed et al. 1987; Doris and Gray 1978a; Hua et al. 1993a; Kamdem et al. 1991; Koubaa et al. 1996; Liu and Rials 1998; Ostmeyer et al. 1988). The carbon C1s peak in the XPS spectra of the wood surface contains three main components: $\mathrm{C} 1, \mathrm{C} 2$, and $\mathrm{C} 3$. The $\mathrm{C} 1$ component is comprised of carbon atoms bound only to other carbon atoms or hydrogen atoms. The $\mathrm{C} 2$ component is composed of carbon atoms bound to a single non-carbonyl oxygen atom in addition to another carbon or hydrogen atoms. The $\mathrm{C} 3$ component represents carbon atoms bonded to another carbon atom and hydrogen atoms, plus either: (1) one carbonyl oxygen atom, or (2) two non-carbonyl oxygen atoms. In some cases of wood analysis, XPS detected even C4. This component is carbon linked to a carbonyl and a non-carbonyl. 
Young et al. (1982) stated that $\mathrm{C} 1$ is related to carbon-carbon and carbon-hydrogen bonds in extractives and lignin. The bonds involving $\mathrm{C} 2$ can result from all three classes of wood components, but predominantly in the carbohydrates as $-\mathrm{CHOH}$ and in lignin as $\beta$-ether and $-\mathrm{C}$ $\mathrm{OH}$ bonds. $\mathrm{C} 3$ carbon atoms occur as carbonyl groups of the lignin and as the carbon atom bonded to two oxygen atoms of polysaccharides. Table 2.1 presents possible variations of $\mathrm{C} 1 \mathrm{~s}$ atom of a wood surface (Kazayawoko et al. 1998).

Table 4.1. Possible components of Cls peak of wood, type of bonding, and binding energy.

\begin{tabular}{|c|c|c|}
\hline Type of Carbon & Bonding & Binding energy $(\mathrm{eV})$ \\
\hline $\mathrm{C} 1$ & $\mathrm{C}-\mathrm{C}$ or $\mathrm{C}-\mathrm{H}$ & $285.0 \pm 0.4$ \\
\hline $\mathrm{C} 2$ & $\mathrm{C}-\mathrm{O}$ & $285.6 \pm 0.4$ \\
\hline $\mathrm{C} 3$ & $\mathrm{C}=\mathrm{O}$ or $\mathrm{O}-\mathrm{C}-\mathrm{O}$ & $288.0 \pm 0.4$ \\
\hline $\mathrm{C} 4$ & $\mathrm{O}-\mathrm{C}=\mathrm{O}$ & $289.5 \pm 0.4$ \\
\hline
\end{tabular}

\subsubsection{The O/C Ratio and the C1/C2 Ratio of Cellulose and Hemicelluloses}

Cellulose consists of anhydroglucopyranose units, which are bound by $\beta$-(1-4)-glycosidic linkages. The repeating unit of the cellulose chain is actually a cellobiose unit (Figure 4.1). In terms of the $\mathrm{O} / \mathrm{C}$ ratio, one can see that one cellobiose has 10 oxygen atoms and 12 carbon atoms. Therefore, the theoretical atomic $\mathrm{O} / \mathrm{C}$ ratio for cellulose is 0.83 . Hemicelluloses have an $\mathrm{O} / \mathrm{C}$ ratio close to that of cellulose. For instance, xylan and galctoglucomannan have the O/C ratio of 0.8 and 0.81 , respectively (Börås and Gatenholm 1999).

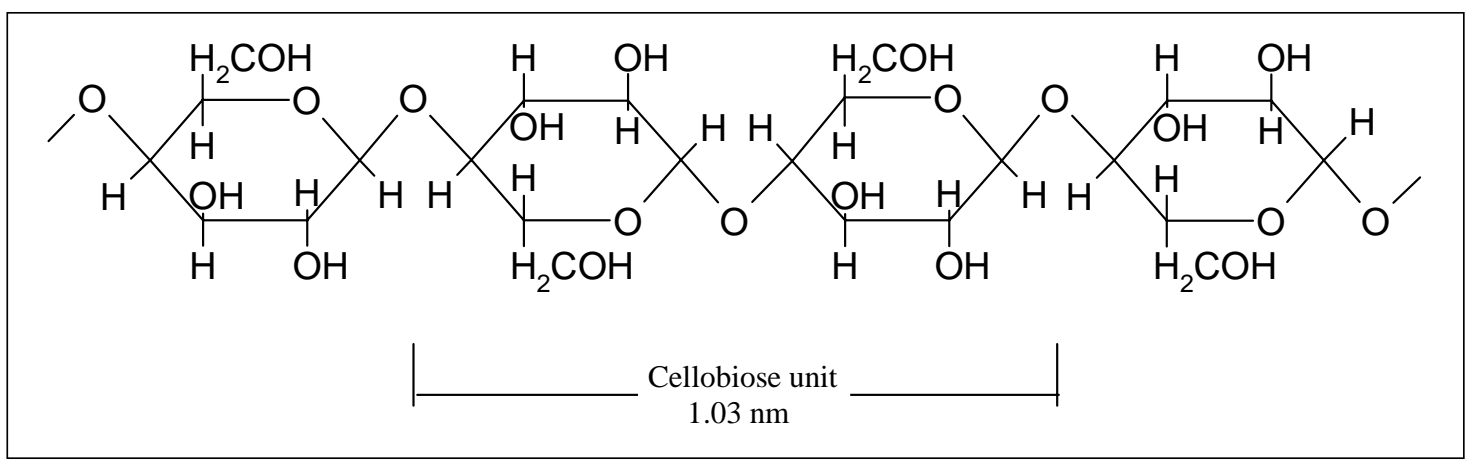

Figure 4.1. Formula of cellulose. 
The assignments of the curve fit $\mathrm{C} 1 \mathrm{~s}$ peak demonstrated that, generally, a pure cellulose has two different types of carbon bonded to other elements. Theoretically, there are $0 \% \mathrm{C} 1,83 \%$ C2, and 17\% C3 (Hua et al. 1993a). Therefore, the C1/C2 ratio for pure cellulose equals 0 . When analyzing wood cellulose, XPS often detects a small amount of the C1 type of carbon because of impurities or contamination of the sample. Thus, the measured $\mathrm{C} 1 / \mathrm{C} 2$ ratio of wood cellulose is often a bit higher than 0 . The percent of C1, C2 and C3 type of carbons in hemicelluloses is close to that of cellulose because the carbon bond structure is similar in both constituents (Hua et al. 1993a). The binding energy of C1, C2, and C3 on the cellulose surface is 284.9-285.0, 286.5286.7 and 288.3-288.5eV, respectively (Hon 1984; Hua et al. 1993a).

\subsubsection{The O/C Ratio and the C1/C2 Ratio of Lignin}

Lignin is a polymer of phenylpropane units. The primary precursors of lignin are coniferyl alcohol (II) in gymnosperms, sinapyl alcohol (III), and p-coumaryl alcohol (I) in angiosperms (Figure 4.2). All these precursors are derivatives of cinnamyl alcohol (Sjöström 1993). The theoretical $\mathrm{O} / \mathrm{C}$ ratio for lignin precursors is $2 / 9$ for $\mathrm{p}$-coumaryl alcohol, $3 / 10$ coniferyl alcohol, and 4/11 for sinapyl alcohol. Klason lignin has an O/C ratio of about 0.33 (Barry et al. 1990). Lignin has three types of carbon C1, C2 and C3. According to Freudenberg's spruce lignin empirical model, lignin contains $49.2 \%$ of $\mathrm{C} 1$ component, $48.8 \%$ of $\mathrm{C} 2$ and $2 \%$ of C3 (Hua et al. 1993a). Therefore, the theoretical C1/C2 ratio of lignin is close to unity.

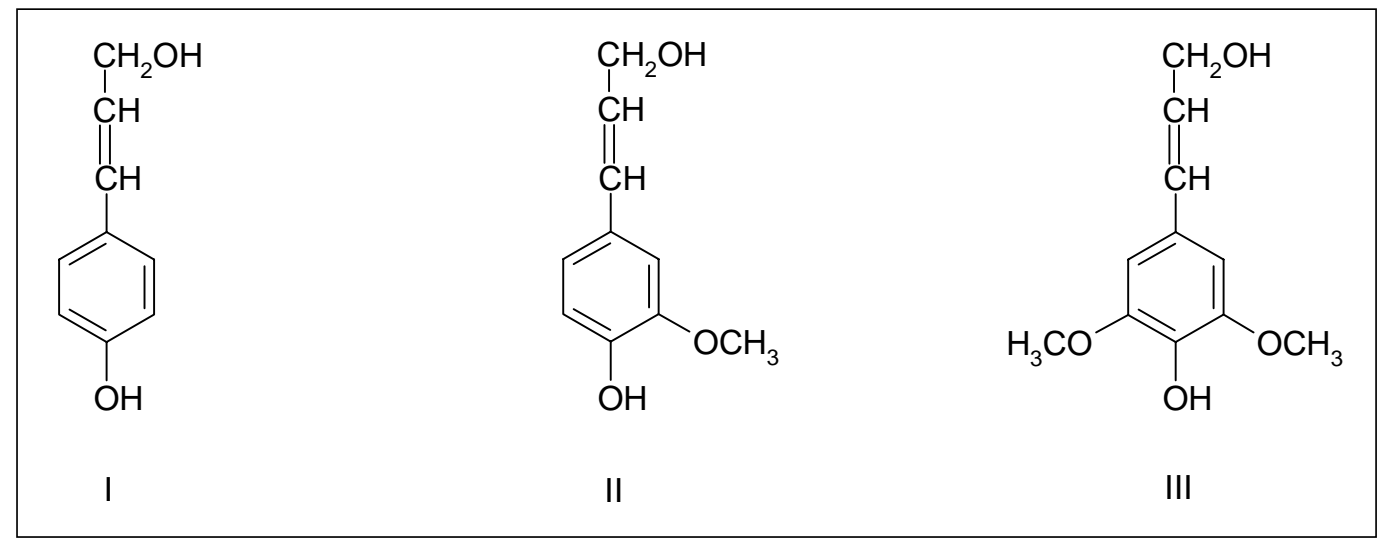

Figure 4.2. Lignin precursors: p-coumaryl (I), coniferyl (II), and sinapyl (III) alcohols. 


\subsubsection{The O/C Ratio and the C1/C2 Ratio of Extractives}

Wood extractives are usually low-molecular-weight organics produced in the living tree. Different compounds of wood extractives can be extracted from wood by means of polar and non-polar solvents. In a narrow sense, extractives comprise the compounds which are soluble in organic solvents. However, water-soluble carbohydrates and inorganic matter also belong to the extractable substances (Fengel and Wegener 1989).

The content and composition of wood extractives vary among wood species and within the species (Hillis 1987). Bark and heartwood usually contain higher extractive concentration than sapwood. Trees from temperate zones often have less extractive content than tropical trees. Domestic woods may contain $2-25 \%$ of extractives, depending on species and solvent used for extraction. For instance, southern pine contains 3.5-5.4\% of extractives, while yellow-poplar contains 2.4-3.8\% (Rowe 1989; White 1987). Many wood extractives are hydrocarbons or their derivatives, which are comprised of terpenes, terpenoids, fats, waxes, fatty acids, alcohols, etc. (Fengel and Wegener 1989). Other extractives are carbohydrates. One classification of wood extractives distinguishes among (Fengel and Wegener 1989; Helm 2002):

- volatile oils existing mainly in softwoods (e.g., monoterpenes);

- wood resins existing mainly in softwoods (e.g., acidic diterpenes);

- fats and waxes (e.g., fatty acids esters and suberin);

- tannins (e.g., hydrolysable and condensed);

- lignans (e.g., syringaresinol);

- carbohydrates (e.g., starch); and

- $\quad$ proteins and peptides (e.g., amino acids).

\subsubsection{Extractives of Yellow-Poplar}

Yellow-poplar extractives consist of alkaloids, sesquiterpenes, and lignans (USDA 1979). Alkaloids are comprised of aporphine, glaucine, dehydroglaucine, norglaucine, and liriodenine. The yellowish-green color of normal heartwood of yellow-poplar originates from liriodenine (Figure 4.3, left) and O-methylatheroline (USDA 1979). 


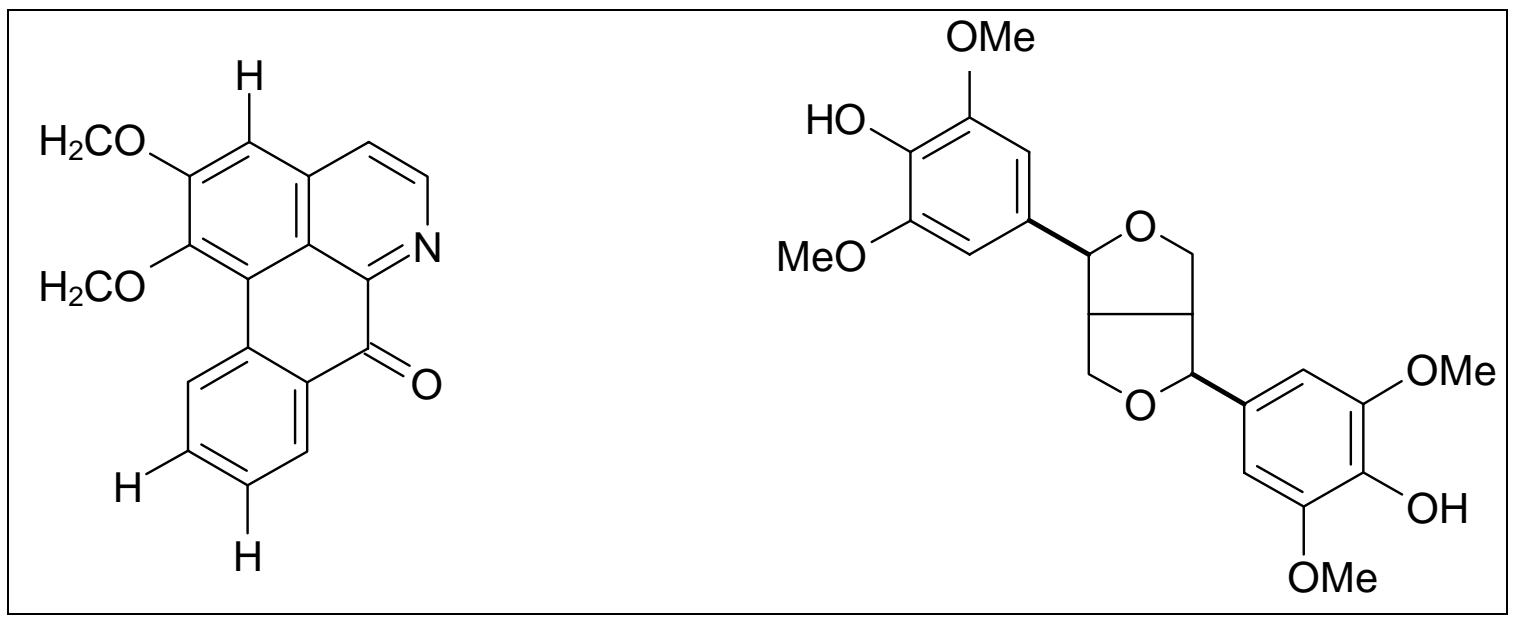

Figure 4.3. Yellow-poplar alkaloid liriodenine (left), and lignan syringaresinol (right).

Alkaloids are toxic, which provides resistance to decomposition by fungi, bacteria, and insects (Rowell 1984). The original meaning of alkaloid is " alkali-like substance from nature" (Rowe 1989). Many alkaloids are basic, but not all. The sesquiterpenes of yellow-poplar are located mainly in bark or roots (USDA 1979). Lignans of yellow-poplar are comprised of syringaresinol (Figure 4.3, right), syringaresinol dimethyl ether, liriodendrin, medioresinol, etc. (Hillis 1962; USDA 1979). Lignans are found mostly in heartwood, where they are deposited during heartwood formation. When purified, lignans are typically crystalline products with a melting point range from $64^{\circ} \mathrm{C}$ to $250^{\circ} \mathrm{C}$ (Northey 2002). The lignans liriodendrin and syringaresinol have high melting points of $270^{\circ} \mathrm{C}$ and $235^{\circ} \mathrm{C}$, respectively (Hillis 1962). Lignans are nonvolatile products that can be extracted from wood with hot water, polar solvents, ether, aromatic solvents, and alkalis (Northey 2002).

The $\mathrm{O} / \mathrm{C}$ and $\mathrm{C} 1 / \mathrm{C} 2$ ratio can vary substantially among yellow-poplar extractives. For instance, the theoretical $\mathrm{O} / \mathrm{C}$ ratio for liriodenine is about 0.15 , which is low, while the theoretical $\mathrm{O} / \mathrm{C}$ ratio of syringaresinol is 0.36 , which is higher than the $\mathrm{O} / \mathrm{C}$ ratio of most extractives. The theoretical $\mathrm{C} 1 / \mathrm{C} 2$ ratio for liriodenine is 3 and for syringaresinol is 0.57 . 


\subsubsection{Extractives of Southern Pine}

Southern pine extractives consist of terpenes and terpenoids, resin acids, fats, fatty acids, organic acids, phenolic compounds, and sugars (Stanley 1969). Terpenes can be classified into monoterpenes, sesquiterpenses, diterpenes, etc. All of them are hydrocarbon derivatives of isoprene (i.e., 2-methyl butadiene). They differ in the number of isoprene units linked in the terpene. Some authors do not distinguish between terpenes and terpenoids. However, the name "terpene" refers to unsaturated terpene hydrocarbons (Zavarin and Cool 1991).

The monoterpenes consist of two isoprene units and are abundant in the pines. Stanley (1969) reported that most common monoterpenes of southern pine (e.g., loblolly pine) are $\alpha$ pinene (71\%), $\beta$-pinene (22\%), and limonene (1\%) (Figure 4.4). A similar composition of loblolly pines monoterpenes was found in other studies (Fengel and Wegener 1989).

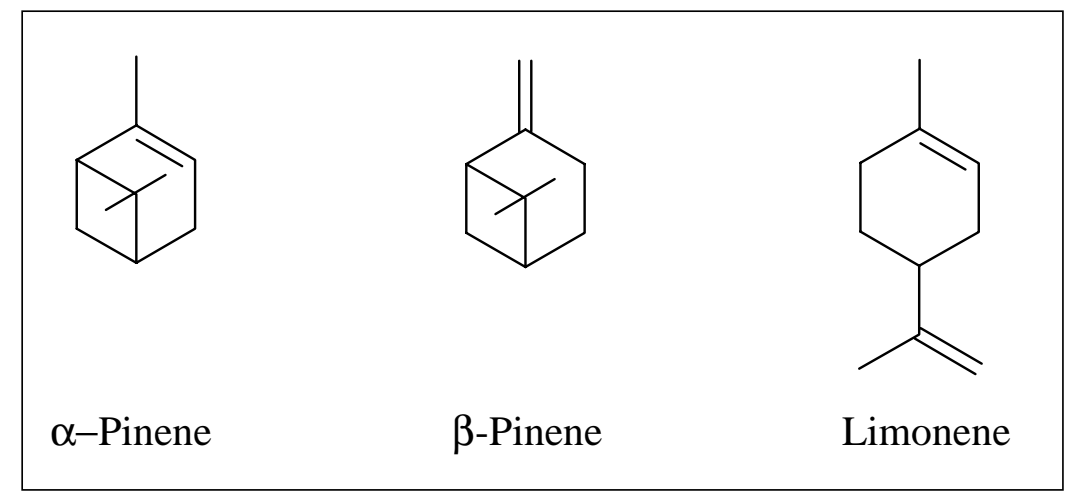

Figure 4.4. Common monoterpenes of southern pine.

At room temperature, some monoterpenes are liquids and some are solids with a low melting point. For instance, at $101.3 \mathrm{kPa} \alpha$-pinene has a melting point at $-50^{\circ} \mathrm{C}$ and a boiling point at $156^{\circ} \mathrm{C}$ (Northey 2002). The main part of turpentine (i.e., volatile wood oil) is comprised of monoterpenes (Fengel and Wegener 1989).

Diterpenes (four isoprene units), which are soluble in petroleum ether, are the main part of the softwood oleoresin (Northey 2002). The neutral diterpenes consist of hydrocarbons, oxides, alcohols, and aldehydes (Fengel and Wegener 1989). The acid diterpenes, which typically are referred to as resin acids (Figure 4.5), are mainly located in heartwood. 


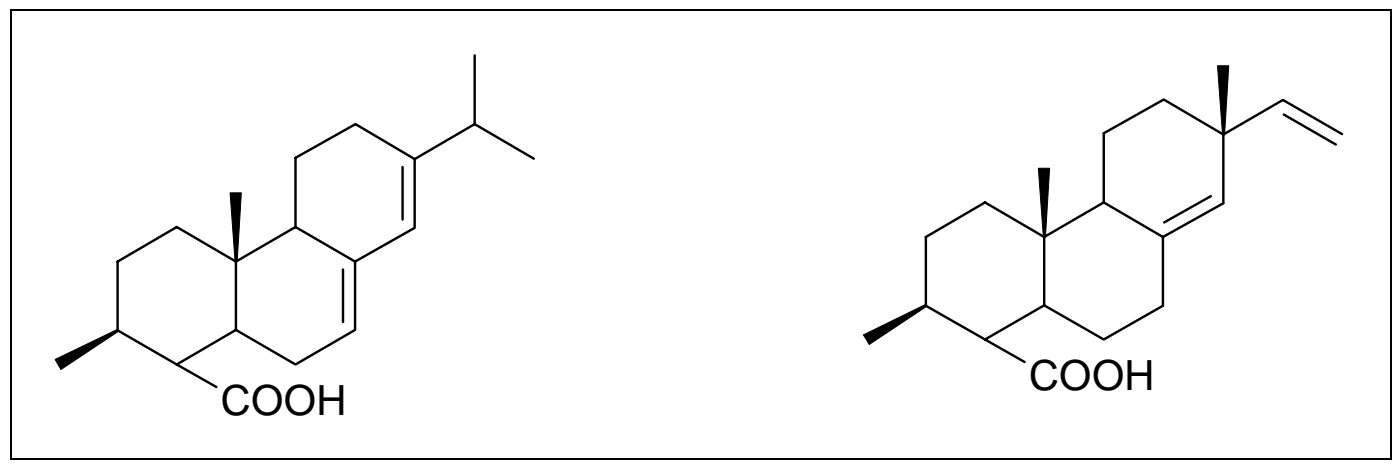

Figure 4.5. Typical resin acids in southern pine: abietic (left) and pimaric (right).

Pure resin acids are crystalline. They are nonvolatile components of the pine's oleoresin and canal resin (Hillis 1962). Resin acids usually have high boiling points. An abietic and a pimaric acid have boiling points at $172-175^{\circ} \mathrm{C}$ and $217-219^{\circ} \mathrm{C}$, respectively (Hillis 1962).

A distillation of resinous materials produces rosin, which is mostly a mixture of diterpenoids' resin acids. Typical wood rosin consists of pimaric (3\%), palutric (10\%), isopimeric (7\%), abietic (35\%), dehydroabietic (20\%), and neoabietic (4\%) diterpenes (Northey 2002). Rosin is glass like, but it can crystallize when one constituent comprises more than 25$30 \%$. Besides terpenes, southern pine extractives are composed of a small amount of free fatty acids, combined fatty acids, glycerol, free sterols, sterols, lignans, and phenolic compounds (Stanley 1969).

The $\mathrm{O} / \mathrm{C}$ ratio of monoterpenes and diterpenes is 0 , since there are no oxygen atoms in typical unsaturated terpenes. Monoterpenes contain 10 carbons whereas diterpenes contain 20 carbons. The $\mathrm{O} / \mathrm{C}$ ratio of terpenoids is low. Abietic acid, which is the most common diterpenoid resin acid, has an $\mathrm{O} / \mathrm{C}$ ratio of 0.10 . An oleic acid has the theoretical $\mathrm{O} / \mathrm{C}$ ratio of 0.11 (Börås and Gatenholm 1999). The C1/C2 ratio of monoterpenes and diterpenes is very high, since the main component of a carbon atom is $\mathrm{C} 1$ type (i.e., $\mathrm{C}-\mathrm{C}$ or $\mathrm{C}-\mathrm{H}$ ). An abietic acid has $95 \%$ of the C1 component (Hua et al. 1993a). 


\subsubsection{Hydrocarbon and Carbohydrate Types of Extractives}

Wood extractives are classified in many ways. Chemical classification, which is simple and the most often used, is based on the overall similarity of the chemical structures of the extractive components (Zavarin and Cool 1991). The classification of the extractives according to analysis groups is presented in Table 4.2.

Table 4.2. Classification of the extractives with examples according to analysis groups (Fengel and Wegener 1989).

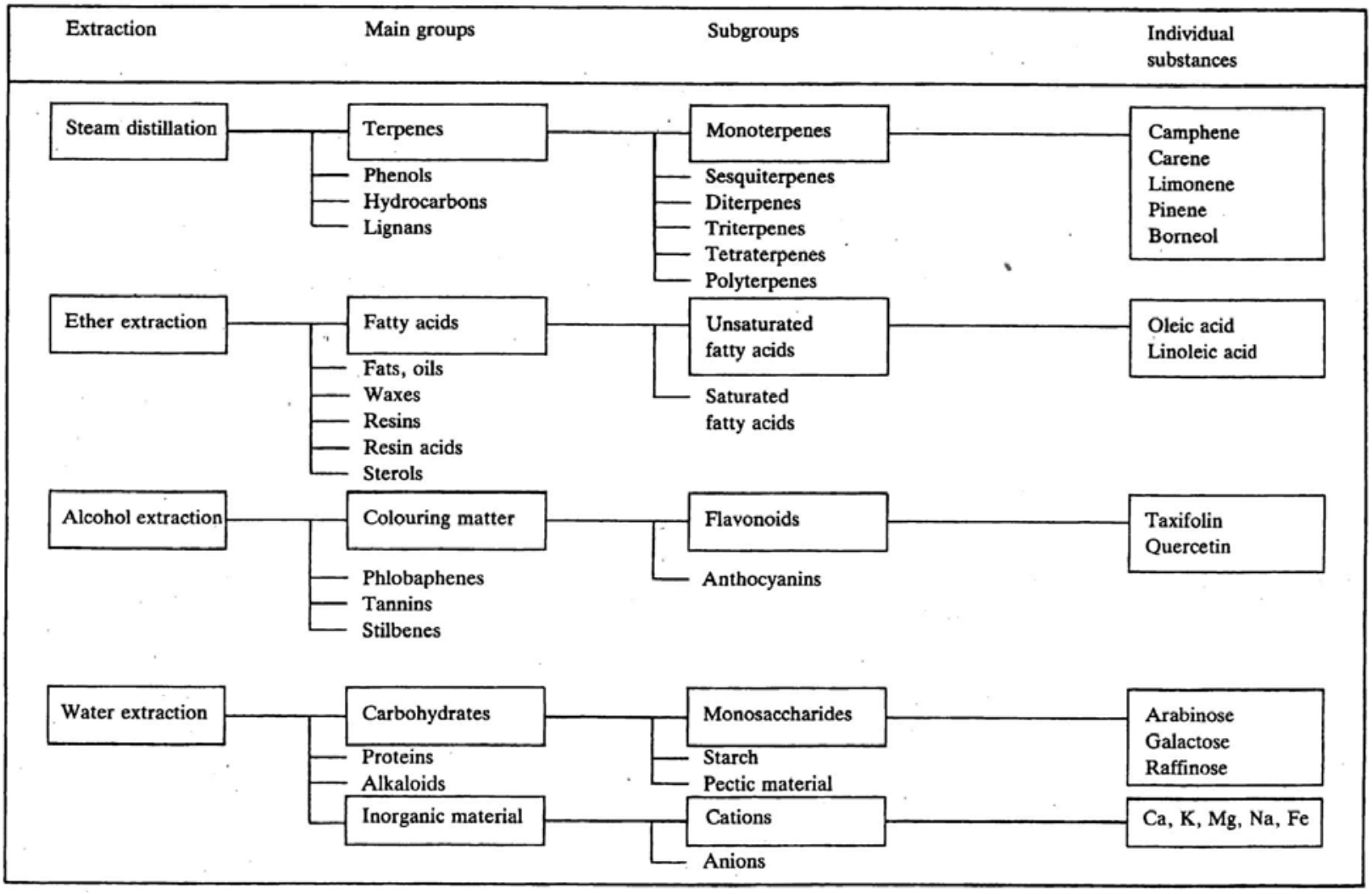

For the purpose of this study, extractives of yellow-polar and southern pine were roughly classified as hydrocarbon (HC) type extractives and carbohydrate $(\mathrm{CH})$ type extractives. The classification of extractives into the HC-type and CH-type was based on Table 4.2 and on the analysis of the results in several studies (Hillis 1962; Manninen et al. 2002; McGraw et al. 1999; Mohseni and Allen 2000; Rowell 1984; Widsten et al. 2002; Wu and Milota 1999). 
The HC-type extractives include hydrocarbons and their derivatives, and the CH-type extractives include carbohydrates and their derivatives. This classification cannot include all extractives, but it gives some valuable information on extractive polarity or non-polarity and hydrophilicity or hydrophobicity. In general, heartwood of yellow-poplar is comprised of more CH-type extractives, which are polar and usually hydrophilic. On the other hand, extractives of southern pine heartwood are mostly of the HC-type, non-polar, and hydrophobic (Table 4.3).

Table 4.3. Type and properties of relevant yellow-polar and southern pine extractives.

\begin{tabular}{|c|c|c|c|c|}
\hline HC Type & $\leftarrow$ & more & $\rightarrow$ & СН Type \\
\hline \multirow{2}{*}{\multicolumn{2}{|c|}{$\begin{array}{c}\text { Non-polar } \\
\text { Hydrophobic }\end{array}$}} & Non-polar/Polar & \multirow{2}{*}{\multicolumn{2}{|c|}{$\begin{array}{c}\text { Polar } \\
\text { Usually Hydrophilic }\end{array}$}} \\
\hline & & & & \\
\hline \multirow{2}{*}{$\begin{array}{c}\text { Monoterpenes (SP) } \\
\text { Diterpenes (SP) }\end{array}$} & Fatty acids & Sesquiterpenes & Alkaloids (YP) & Some lignans \\
\hline & (SP) & (YP) & Lignans (YP) & (YP Liriodendrin) \\
\hline
\end{tabular}

The HC-type extractives can be further divided based on emissions into the condensed $\mathrm{HC}$ with higher molecular weight and the low molecular weight $\mathrm{HC}$, which generally is referred to as volatile $\mathrm{HC}$. The condensed HC extractives consist of diterpenoids, some sesquiterpenoid compounds, and undefined oxidation products. The volatile part of HC extractives (i.e., gaseous) is comprised of monoterpenes and some of their oxidation products (McGraw et al. 1999).

Yellow-poplar contains a small amount of volatile organic compounds (VOCs). Southern pines, however, contain a substantial amount of VOCs, emissions of which are by an order of magnitude higher than in hardwoods (Banerjee 2001). VOCs of southern pine are principally terpenes that consist mainly of $\alpha$-pinene and $\beta$-pinene (Baumann et al. 1999).

\subsubsection{Objectives}

In order to provide evidence for the postulated inactivation mechanisms in the previous chapter, the elemental and chemical composition of isolated wood constituents, and of the extracted and unextracted wood surfaces, was determined. The relationship between surface chemistry and wettability was studied. The improvement in adhesion due to the extraction of the wood samples was also evaluated. 


\subsection{Materials}

\subsubsection{Materials and Preparation of Samples}

This experiment initially analyzed the surface chemistry of five wood constituents. The study comprised a model film of cellulose, a yellow poplar lignin powder, a film of yellow poplar extractives, a film of southern pine extractives, and a film of consolidated SP pitch resin (Table 4.4).

\section{Table 4.4. Preparation of wood constituents.}

\begin{tabular}{|c|c|c|}
\hline Sample & Constituent & Preparation \\
\hline CELL & Cellulose film & Langmuir-Blodgett film from TMS cellulose dissolved in chloroform \\
\hline LIGNIN & Lignin, YP & Lignin powder, $\log \mathrm{R}_{0} 4.1$, pressed into pellets and cut with razor blade \\
\hline EXT-YP & Extractives, YP & Extracted with acetone:water $(9: 1)$ from sawdust, film dried at $50^{\circ} \mathrm{C}$ \\
\hline EXT-SP & Extractives, SP & Extracted with acetone:water $(9: 1)$ from sawdust, film dried at $50^{\circ} \mathrm{C}$ \\
\hline RESIN & Pitch resin, SP & Resin melted at temperature $>150^{\circ} \mathrm{C}$ for 1 hour and consolidated \\
\hline
\end{tabular}

The cellulose film model (CELL) was prepared according to Löscher et al. (1998). The CELL sample was supplied from a parallel study (Jamin 2001). The Langmuir-Blodgett technique was used to form a uniform, monomolecular cellulose layer from trimethylsilyl (TMS) cellulose. Around $5 \mathrm{mg}$ TMS cellulose was dissolved in $10 \mathrm{ml}$ chloroform. A $60 \mu \mathrm{l}$ volume of the solution was sprayed onto the water surface. The surface pressure of cellulose molecules on the water surface was recorded for detecting a point when the film achieved a condensed liquid state $(10-20 \mathrm{mN} / \mathrm{m})$. The dipping of the glass slide through the monolayer/air interface produced one layer thick cellulose film. This procedure was repeated to achieve 6 layers stacked on top of each other. The cellulose film was then desilylated by exposure to $\mathrm{HCl}$ vapor.

A lignin sample (LIGNIN) was taken from previous research (Glasser et al. 1993), which was produced by a steam explosion process performed by the B-REAL Company of Floyd, Virginia. Lignin powder, which was isolated from yellow-poplar (Angiolin, $\log R_{0} 4.1,4 / 1990$ ), was pressed into pellets and then cut by a razor blade into a small cube $(6 \times 6 \times 6 \mathrm{~mm})$ prior to XPS analysis. 
Yellow-poplar extractives (EXT-YP) and southern pine extractives (EXT-SP) were prepared separately in two beakers. Since the extraneous materials cannot be completely removed from wood by a single solvent (Rowell 1984), a combination of several solvents is often used. In many cases, acetone-water was a suitable solvent for removing polyphenolic extractives from wood (Hillis 1962). Therefore, five grams of sawdust was immersed in $200 \mathrm{ml}$ acetone:water solution (9:1, by volume) for 24 hours. The beakers were then placed on a heater to accelerate extraction due to increased extractives solubility. Boiling at $56^{\circ} \mathrm{C}$ for 1 hour removed around $95 \%$ of the solvents. The extract was filtered and then placed on a glass slide. Evaporating the excess solvent formed a thin film of extractives. This sample was dried at $50^{\circ} \mathrm{C}$ for 20 minutes in a convection oven for the complete removal of the solvents. In addition to these two extracts, a small amount of southern pine resin (RESIN) was collected and placed on a glass slide. Resin was melted and consolidated by exposure to temperature $>150^{\circ} \mathrm{C}$ for one hour. The color of the resin turned from yellow to light brown at the end of the consolidation.

Besides wood constituents, this experiment also investigated heartwood of yellow-poplar (Liriodendron tulipifera), and southern pine (Pinus taeda). The wood samples, with green moisture content, were cut to a rectangular shape $(165 \times 60 \mathrm{~mm})$ and planed to a thickness of 10 mm. The tangential wood surface was studied. The treatment of the yellow-poplar (YP) and southern pine (SP) samples was comprised of wood drying, and/or solvent extraction (Table 4.5).

Table 4.5. Treatments of wood samples.

\begin{tabular}{|c|c|c|}
\hline Sample & Species & Preparation/Treatment \\
\hline YP50EXT & Yellow-poplar & Drying at $50^{\circ} \mathrm{C}$, then extraction with acetone:water $(9: 1)$ for 6 weeks \\
\hline YP200 & Yellow-poplar & Drying at $200^{\circ} \mathrm{C}$, without extraction \\
\hline YP200EXT & Yellow-poplar & Drying at $200^{\circ} \mathrm{C}$, then extraction with acetone:water $(9: 1)$ for 6 weeks \\
\hline YPEXT200 & Yellow-poplar & Extraction with acetone:water $(9: 1)$ for 6 weeks, then drying at $200^{\circ} \mathrm{C}$ \\
\hline SP50EXT & Southern pine & Drying at $50^{\circ} \mathrm{C}$, then extraction with acetone:water $(9: 1)$ for 6 weeks \\
\hline SP200 & Southern pine & Drying at $200^{\circ} \mathrm{C}$, without extraction \\
\hline SP200EXT & Southern pine & Drying at $200^{\circ} \mathrm{C}$, then extraction with acetone:water $(9: 1)$ for 6 weeks \\
\hline SPEXT200 & Southern pine & Extraction with acetone:water $(9: 1)$ for 6 weeks, then drying at $200^{\circ} \mathrm{C}$ \\
\hline
\end{tabular}


Wood samples were dried in a convection oven either at $50^{\circ} \mathrm{C}$ for $17 \mathrm{hrs}$ and $45 \mathrm{~min}$., or at $200^{\circ} \mathrm{C}$ for $2 \mathrm{hrs}$ and $15 \mathrm{~min}$, to achieve a final $\mathrm{MC}$ of $2 \%$. The typical increase in wood surface temperature during drying is shown in Figure 4.6.

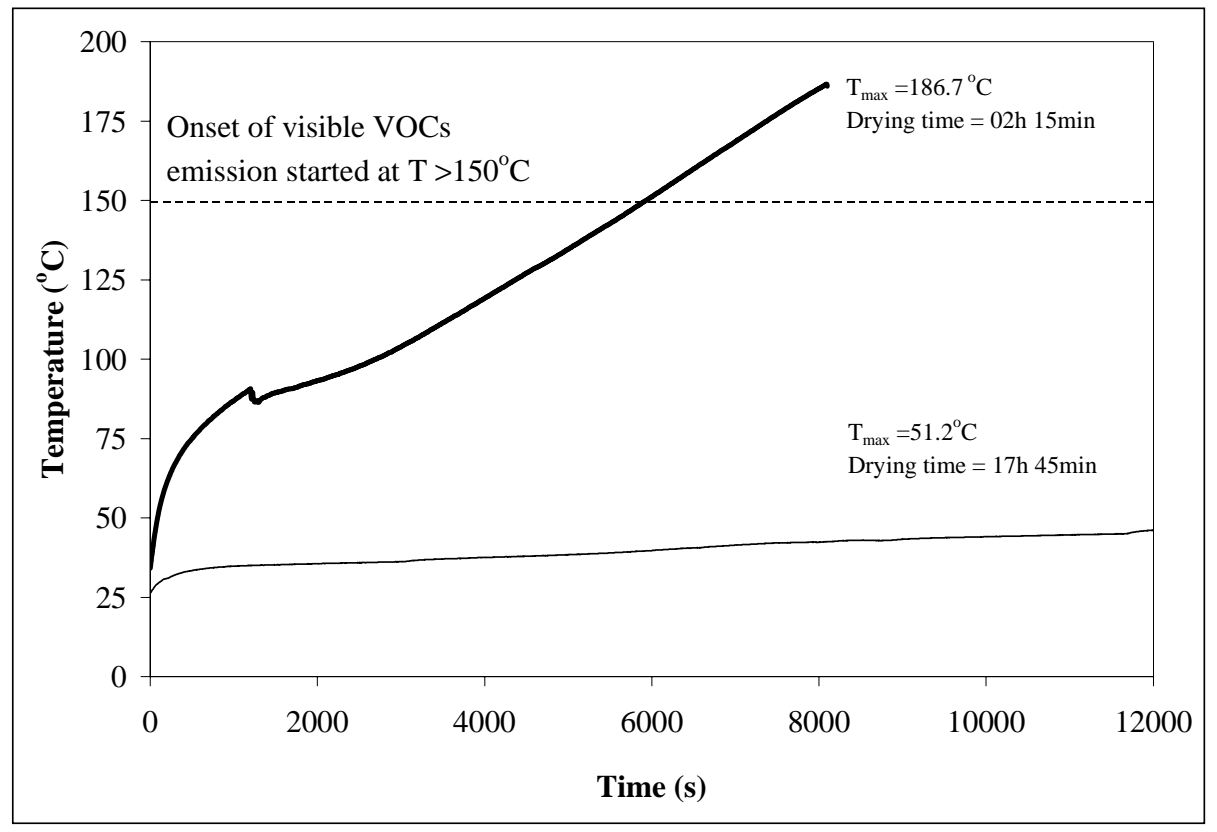

Figure 4.6. Typical increase of wood surface temperature during drying.

The extraction was performed before or after the drying. Samples were extracted with 5 liter acetone:water (9:1) solution in a Soxhlet extractor (Figure 4.7). Because of the large sample dimensions (i.e., 165x60 mm), the samples were extracted for 6 weeks. After extraction, the samples were exposed to the air for 24 hours, which allowed the remaining acetone to evaporate. Extracted samples, which were dried at $200^{\circ} \mathrm{C}$ after extraction (YPEXT200 and YPEXT200), were first immersed in water for 3 days to achieve MC above the fiber saturation point (FSP). 


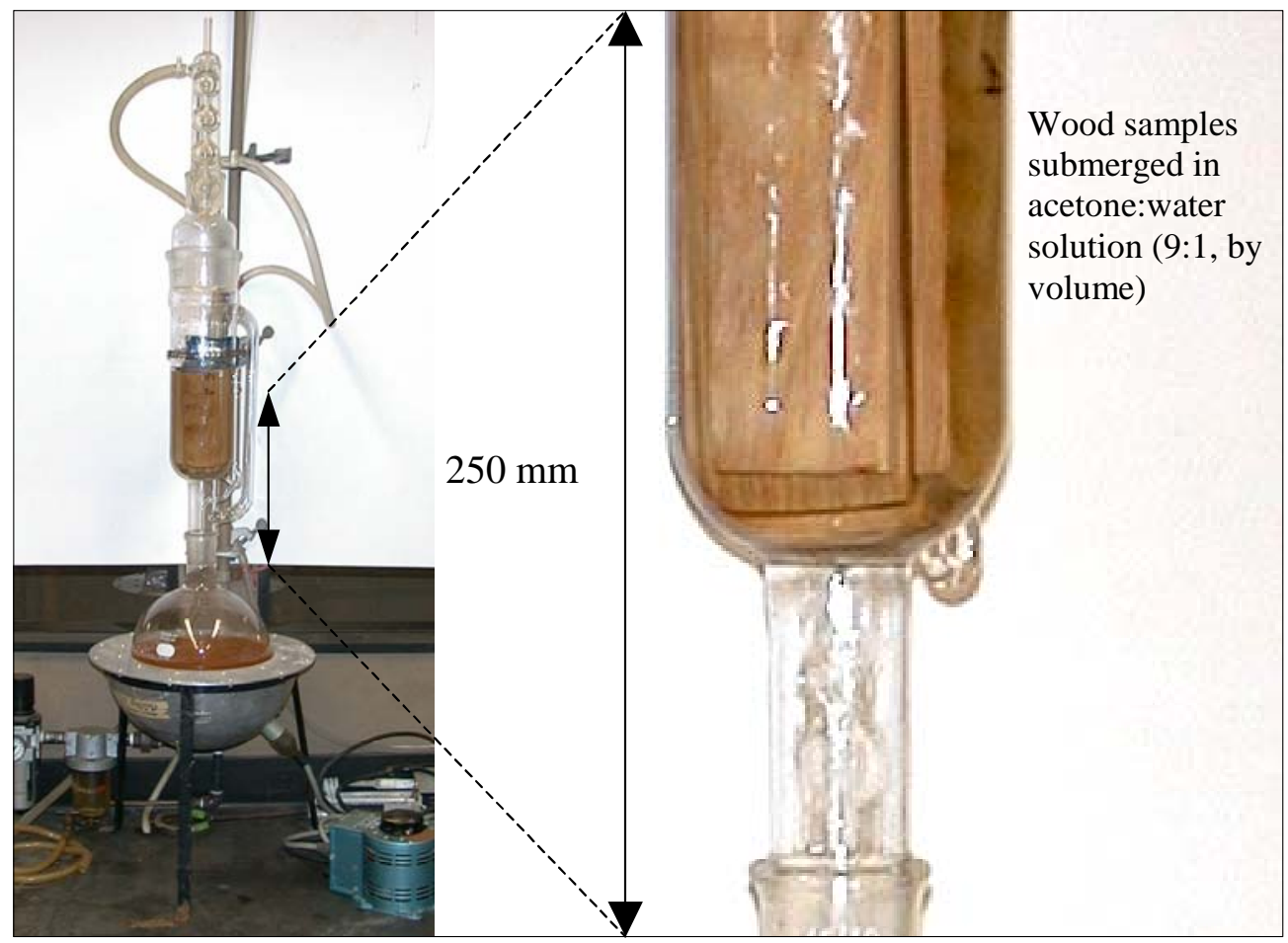

Figure 4.7. Extraction of the wood samples in a big Soxhlet extractor.

\subsubsection{Adhesive and Bonding Parameters}

Thermoset phenol-formaldehyde adhesive (PF) was used to bond together two wood surfaces to evaluate the adhesive bond performance. The PF adhesive Chembond® CB 303, with $\mathrm{pH}$ of 10.5 , was in a liquid state containing $47.1 \%$ of solids. The adhesive was applied to one surface with a roller using a spreading level of $200 \mathrm{~g} / \mathrm{m}^{2}$. The assembly time was one minute. The adhesive was cured in a laboratory press at $200^{\circ} \mathrm{C}$ and $2 \mathrm{~N} / \mathrm{mm}^{2}$ for 15 minutes. The bonded assemblies were then cooled at room temperature for 24 hours. Two specimens for fracture testing were cut from one bonded assembly (discarding the periphery), yielding four fracture specimens per treatment. Prior to testing, the specimens were conditioned for two weeks at 20 $( \pm 2)^{\circ} \mathrm{C}$ and at relative humidity of $65( \pm 2) \%$. 


\subsection{Methods}

The same three experimental methods were used as in Chapter 3. Only the specifications that differed from those in the previous chapter are listed here.

\subsubsection{X-Ray Photoelectron Spectroscopy}

A Perkin-Elmer model $5400 \mathrm{X}$-ray photoelectron spectrometer was employed to provide elemental and chemical data of the surface composition. Two replicate measurements were made on the wood surfaces and only one on the surfaces of cellulose, lignin, and extractives. In total, 21 measurements were performed. The measurements followed the description in section 3.3.1.

\subsubsection{Contact Angle Measurement}

A sessile drop method was used to measure a contact angle $(\theta)$ of a $5 \mu$ distilled water drop. The measurements followed the description in section 3.3.2. The contact angles (i.e., 10 replications) were measured on all wood surfaces and on the cellulose film.

\subsubsection{Fracture Mechanics Test}

The fracture test specimens differ from those used in the previous chapter. The test specimens did not have backing and they were $160 \mathrm{~mm}$ long. The length of the specimens was limited by the extraction procedure, since the Soxhlet apparatus allowed a maximum specimen length of $170 \mathrm{~mm}$. The measurements followed the description in section 3.3.3.

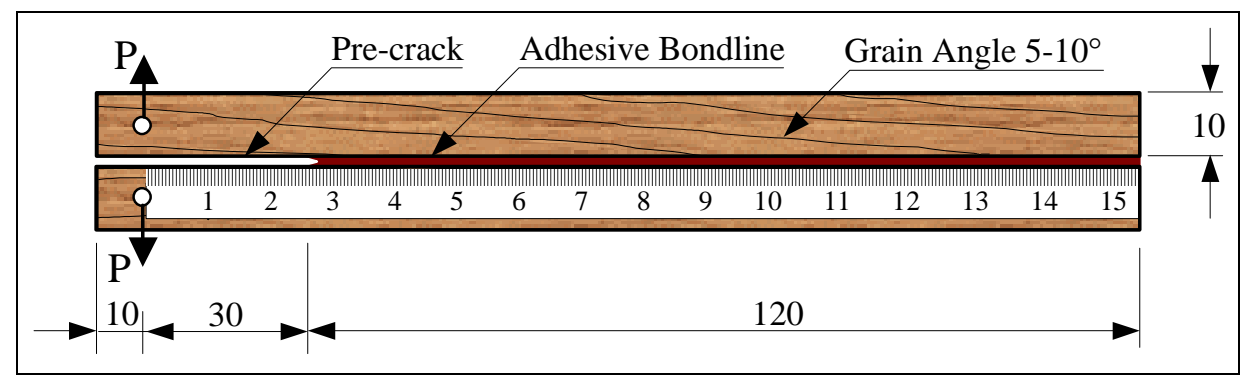

Figure 4.8. Orientation, geometry and dimensions $(\mathrm{mm})$ of the fracture test specimen. 


\subsection{Results and Discussion}

\subsubsection{Chemical Characterization of Wood Surfaces}

Carbon $(\mathrm{C})$, oxygen $(\mathrm{O})$, and nitrogen $(\mathrm{N})$ elements were detected on the investigated surfaces. The atomic percent of the elements and the atomic percent of carbon components (i.e., $\mathrm{C} 1, \mathrm{C} 2$, and C3) were determined from the deconvoluted spectra (Table 2.1).

Table 4.6. Atomic percent of wood and wood constituents as determined by XPS.

\begin{tabular}{|c|c|c|c|c|c|c|}
\hline \multirow[b]{2}{*}{ Sample } & \multicolumn{3}{|c|}{ Elements (\%) } & \multicolumn{3}{|c|}{ Carbon Components (\%) } \\
\hline & $\mathbf{C}$ & $\mathbf{O}$ & $\mathbf{N}$ & C1 & $\mathbf{C 2}$ & $\mathbf{C 3}$ \\
\hline CELL & 57.2 & 42.4 & 0.4 & 14.5 & 65.2 & 20.3 \\
\hline LIGNIN & 71.9 & 27.7 & 0.4 & 49.3 & 44.6 & 6.1 \\
\hline EXT-YP & 81.5 & 17.5 & 1.0 & 74.8 & 21.4 & 3.8 \\
\hline EXT-SP & 87.1 & 12.9 & 0.0 & 82.4 & 13.7 & 3.8 \\
\hline RESIN & 87.2 & 12.9 & 0.0 & 83.4 & 10.2 & 3.5 \\
\hline YP50EXT & 64.2 & 35.3 & 0.5 & 39.4 & 50.1 & 10.4 \\
\hline YP200 & 75.0 & 23.6 & 1.3 & 58.9 & 32.6 & 8.6 \\
\hline YP200EXT & 64.6 & 34.9 & 0.6 & 47.3 & 42.8 & 10.0 \\
\hline YPEXT200 & 68.2 & 29.1 & 2.7 & 54.5 & 35.6 & 9.9 \\
\hline SP50EXT & 65.7 & 31.4 & 2.9 & 48.8 & 39.1 & 12.2 \\
\hline SP200 & 75.1 & 23.5 & 1.4 & 59.4 & 31.0 & 9.6 \\
\hline SP200EXT & 70.0 & 28.4 & 1.6 & 56.1 & 34.8 & 9.1 \\
\hline SPEXT200 & 69.5 & 28.2 & 2.3 & 55.3 & 34.3 & 10.5 \\
\hline
\end{tabular}

For the isolated wood constituents only, the percent of carbon increased and the percent of oxygen decreased in the following order: cellulose, lignin, YP extractives, SP extractives, and SP resin. The percent of nitrogen was low and did not vary significantly among wood constituents. For solid wood samples, SP exhibited higher percent of carbon and lower percent of oxygen than YP. The percent of nitrogen was again low, but in some cases (e.g., YPEXT200, SP50EXT, and SPEXT200) significant. However, the presence of nitrogen on the wood surface was not investigated in detail. The interpretation of the results obtained by XPS analysis of wood material is usually focused on oxygen and especially, carbon atoms. 
Besides the atomic percent, the oxygen to carbon ratio $(\mathrm{O} / \mathrm{C})$ and the $\mathrm{C} 1 / \mathrm{C} 2$ ratio were calculated, since this is a more illustrative way to present changes in $\mathrm{C}$ and $\mathrm{O}$ at the surface. The experimental and some theoretical $\mathrm{O} / \mathrm{C}$ ratios of the analyzed surfaces are shown in Table 4.7.

Table 4.7. O/C and C1/C2 ratios of wood constituents and wood surfaces.

\begin{tabular}{|c|c|c|c|c|}
\hline \multirow[b]{2}{*}{ Sample } & \multicolumn{2}{|c|}{ O/C Ratio } & \multicolumn{2}{|c|}{ C1/C2 Ratio } \\
\hline & Experimental & Theoretical & Experimental & Theoretical \\
\hline CELL & 0.74 & 0.83 & 0.22 & 0 \\
\hline LIGNIN & 0.38 & 0.33 & 1.11 & $\approx 1$ \\
\hline EXT-YP & 0.22 & $\approx 0.15$ & 3.50 & up to 10 \\
\hline EXT-SP & 0.15 & $\approx 0.12$ & 6.00 & High $*$ \\
\hline RESIN & 0.15 & $\approx 0.11$ & 8.14 & High * \\
\hline YP50EXT & 0.55 & & 0.79 & \\
\hline YP200 & 0.31 & & 1.81 & \\
\hline YP200EXT & 0.54 & I & 1.11 & l \\
\hline YPEXT200 & 0.43 & & 1.53 & \\
\hline SP50EXT & 0.48 & & 1.25 & \\
\hline SP200 & 0.31 & & 1.91 & \\
\hline SP200EXT & 0.41 & I & 1.61 & I \\
\hline SPEXT200 & 0.40 & & 1.61 & \\
\hline
\end{tabular}

Cellulose had the highest value of the $\mathrm{O} / \mathrm{C}$ ratio, followed by the $\mathrm{O} / \mathrm{C}$ ratio of lignin, $\mathrm{YP}$ extractives, SP extractives, and SP resin. These results are not surprising, since the theoretical value of $\mathrm{O} / \mathrm{C}$ ratio for cellulose is 0.83 ; while for lignin and extractives it is much lower at 0.33 and 0.10, respectively (Barry et al. 1990). The theoretical interpretation was confirmed by evidence from several studies (Ben et al. 1993; Hon 1984; Mjöberg 1981). According to the theory, a high $\mathrm{O} / \mathrm{C}$ ratio represents a surface containing mostly polysaccharides, while a low $\mathrm{O} / \mathrm{C}$ ratio reflects a high concentration of extractives and lignin. Indeed, the results showed that removal of the extractives increased the $\mathrm{O} / \mathrm{C}$ ratio of the wood surface. A similar extraction effect was found in other studies (Börås and Gatenholm 1999; Doris and Gray 1978b; Kaldas et al. 1998). A graphical illustration of the $\mathrm{O} / \mathrm{C}$ ratio of wood constituents and the effect of the extraction on the $\mathrm{O} / \mathrm{C}$ ratio of wood surfaces is shown in Figure 4.9. 


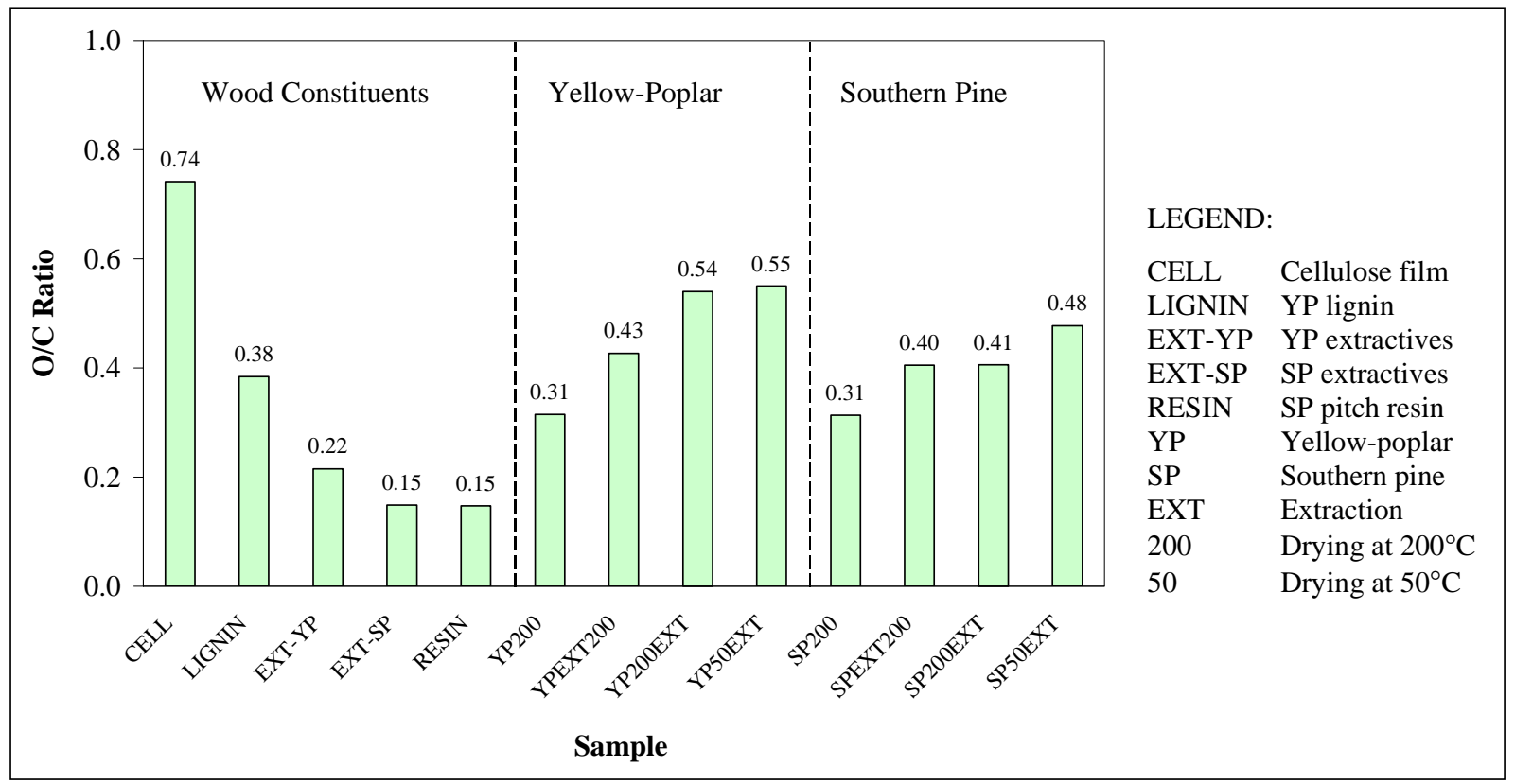

Figure 4.9. O/C ratio of wood and wood constituents.

The difference in the O/C ratio between YP-EXT and SP-EXT was obtained, which was expected. SP contained a high amount of HC-type, non-polar extractives, which all have a very low $\mathrm{O} / \mathrm{C}$ ratio. On the other side, $\mathrm{YP}$ contained mainly $\mathrm{CH}$-type of extractives, the $\mathrm{O} / \mathrm{C}$ ratio of which might range from 0.12 to 0.36 . However, it was unexpected to obtain the same $\mathrm{O} / \mathrm{C}$ ratio for YP200 and SP200 specimens. This O/C was higher than that obtained on the similar surfaces discussed in chapter 3 . The difference might be due to wood variability. For instance, the amount of extractives varies substantially within the tree and among wood species. The surface coverage of extractives $\left(\phi_{\mathrm{e}}\right)$ can be calculated by using the equation (Stenius and Vuorinen 1999):

$$
\phi_{\mathrm{e}}=\frac{\mathrm{O} / \mathrm{C}-\mathrm{O} / \mathrm{C}_{\mathrm{ae}}}{\mathrm{O} / \mathrm{C}_{\mathrm{e}}-\mathrm{O} / \mathrm{C}_{\mathrm{ae}}}
$$

\section{Equation 4.1}

where $\mathrm{O} / \mathrm{C}$ refers to the $\mathrm{O} / \mathrm{C}$ ratio of wood surface before extraction; $\mathrm{O} / \mathrm{C}_{\mathrm{ae}}$ refers to the $\mathrm{O} / \mathrm{C}$ ratio of wood surface after extraction; and $\mathrm{O} / \mathrm{C}_{\mathrm{e}}$ refers to the $\mathrm{O} / \mathrm{C}$ ratio of wood extractives. Using data from this chapter (i.e., the $\mathrm{O} / \mathrm{C}$ ratio before extraction) and from the previous chapter (i.e., the $\mathrm{O} / \mathrm{C}$ ratio after extraction and the $\mathrm{O} / \mathrm{C}$ ratio of extractives), several values for surface coverage of extractives were calculated (Table 4.8). 
Table 4.8. Surface coverage by extractives and VOCs in regard to wood species and drying temperature.

\begin{tabular}{|c|c|c|c|c|}
\hline \multirow{2}{*}{$\begin{array}{l}\text { Wood } \\
\text { Sample }\end{array}$} & \multicolumn{3}{|c|}{ O/C Ratio } & \multirow{2}{*}{$\begin{array}{c}\text { Surface Coverage } \\
(\%)\end{array}$} \\
\hline & Before Extraction & After Extraction & Extractives & \\
\hline YP50/1 & 0.318 & 0.550 & 0.215 & 69 \\
\hline YP200/1 & 0.224 & 0.540 & 0.215 & 97 \\
\hline SP50/1 & 0.250 & 0.477 & 0.149 & 69 \\
\hline SP200/1 & 0.155 & 0.406 & 0.149 & 98 \\
\hline
\end{tabular}

The $\phi_{\mathrm{e}}$ increased about $30 \%$ when the drying temperature was raised from 51 to $187^{\circ} \mathrm{C}$. This confirmed the results from previous studies that temperature accelerates extractive migration to the surface (Hse and Kuo 1988). The increased drying temperature improves solubility of water-soluble extractives, which could then migrate faster to the surface. The diffusion of bound water and steam is increased with increased temperature (Siau 1995).

Water insoluble extractives, which are mostly HC-type, non-polar extractives, migrate to the surface as mass flow or as vapor. Therefore, a certain temperature has to be reached to change the phase of these extractives and accelerate their mobility. At the drying temperature of $187^{\circ} \mathrm{C}$, the wood surface is almost completely covered by extractives/VOCs. This supports statements that extractives form a thin layer on the surface, which then repels water or interferes with resin cure (Hse and Kuo 1988). For the same drying temperature, there was no difference in the $\phi_{\mathrm{e}}$ in regard to wood species. This was not in agreement with the previous interpretation of the $\mathrm{O} / \mathrm{C}$ ratio, which suggested that $\mathrm{SP}$ had a higher amount of extractives on the surface than YP. However, one can realize that the absolute value of the $\mathrm{O} / \mathrm{C}$ ratio for YP extractives was much higher than for SP extractives: 0.215 and 0.149, respectively. Thus, even though $\phi_{\mathrm{e}}$ is similar for both wood species, SP will always exhibit a lower $\mathrm{O} / \mathrm{C}$ ratio.

Calculation of the $\mathrm{C} 1 / \mathrm{C} 2$ ratio (Table 4.7) provided additional evidence in support of the $\mathrm{O} / \mathrm{C}$ interpretation of the wood surface chemistry. The cellulose film had a C1/C2 ratio of 0.22. This is slightly higher than the theoretical $\mathrm{C} 1 / \mathrm{C} 2$ ratio, which is 0 for pure cellulose. However, it is assumed that the small $\mathrm{C} 1$ peak is not attributed to cellulose, but rather to a contamination of the sample (Istone 1995). YP lignin had the C1/C2 ratio of 1.11, which matched perfectly with the theoretical $\mathrm{C} 1 / \mathrm{C} 2$ ratio of lignin. Extractives had the highest $\mathrm{C} 1 / \mathrm{C} 2$ ratio among all observed 
wood constituents. In general, these findings support the interpretation of the chemical composition of the wood surface due to the different drying temperature exposures in the previous chapter, section 3.4.1. A graphical illustration of the $\mathrm{C} 1 / \mathrm{C} 2$ ratio of wood constituents and the effect of the extraction on the $\mathrm{C} 1 / \mathrm{C} 2$ ratio of wood surfaces is shown in Figure 4.10.

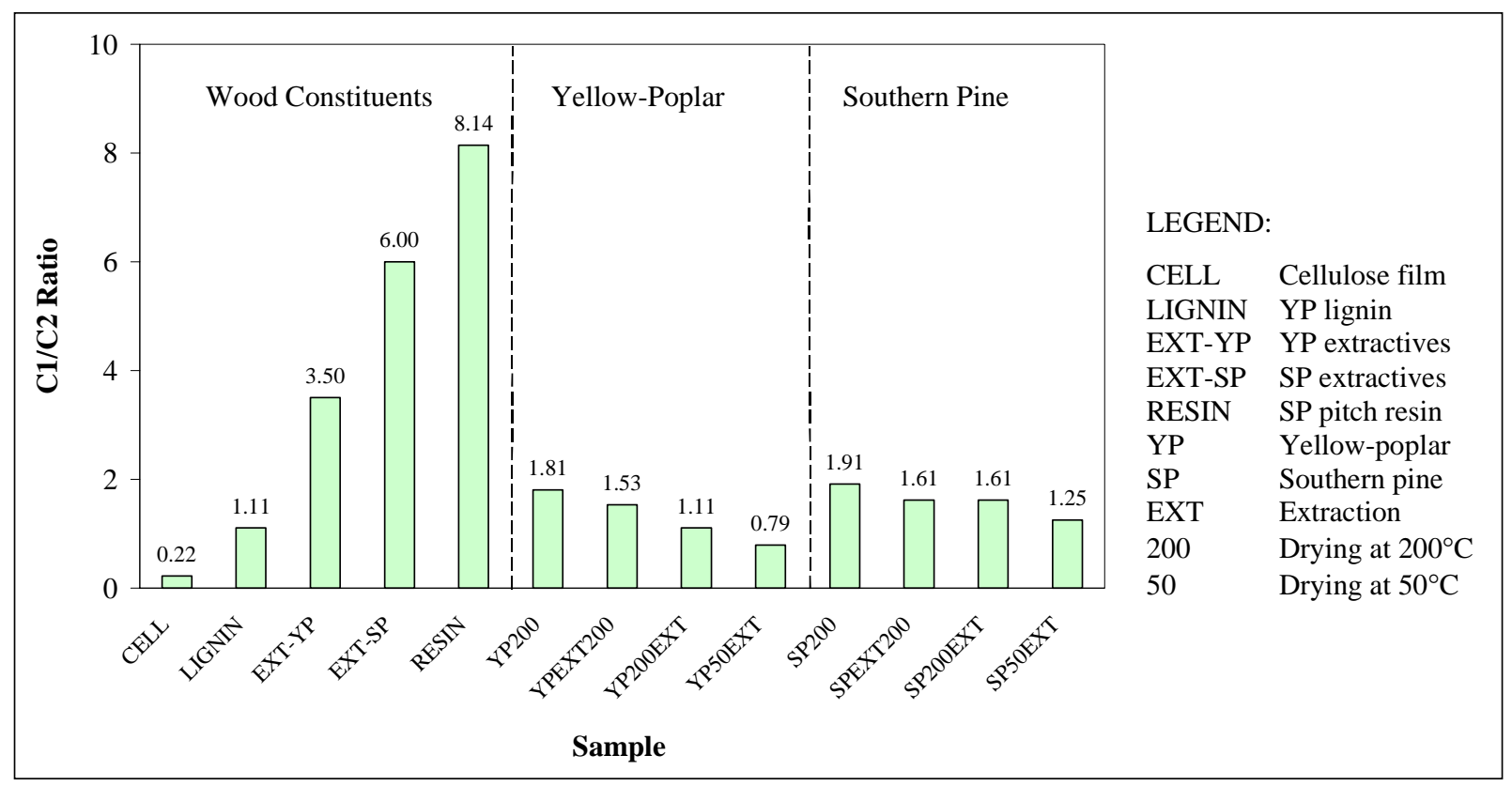

Figure 4.10. C1/C2 ratio of wood and wood constituents.

The $\mathrm{C} 1 / \mathrm{C} 2$ ratio increased in the following order: cellulose, lignin, YP extractives, SP extractives, and SP resin. This relationship can be used to roughly describe wood surface composition - the higher the $\mathrm{C} 1 / \mathrm{C} 2$ ratio, the higher the relative concentration of extractives and lignin on a wood surface (Börås and Gatenholm 1999). Schematic presentation of curve fit for C1s peak of wood constituents is shown in Figure 4.11. Unextracted wood samples exhibited a higher $\mathrm{C} 1 / \mathrm{C} 2$ ratio than the extracted samples, which is illustrated in Figure 4.12. 


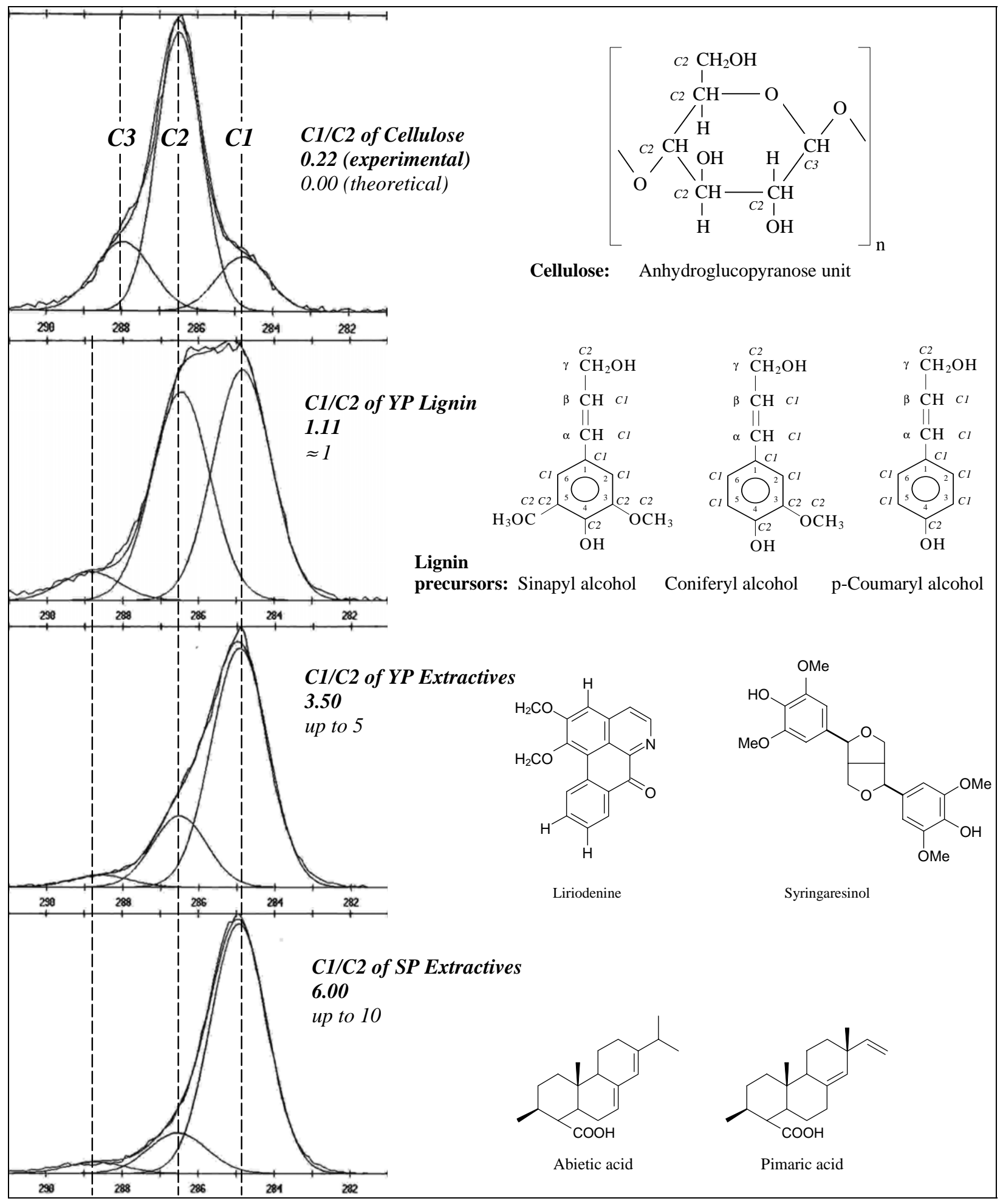

Figure 4.11. Schematic presentation of curve fit for C1s peak of wood constituents. 


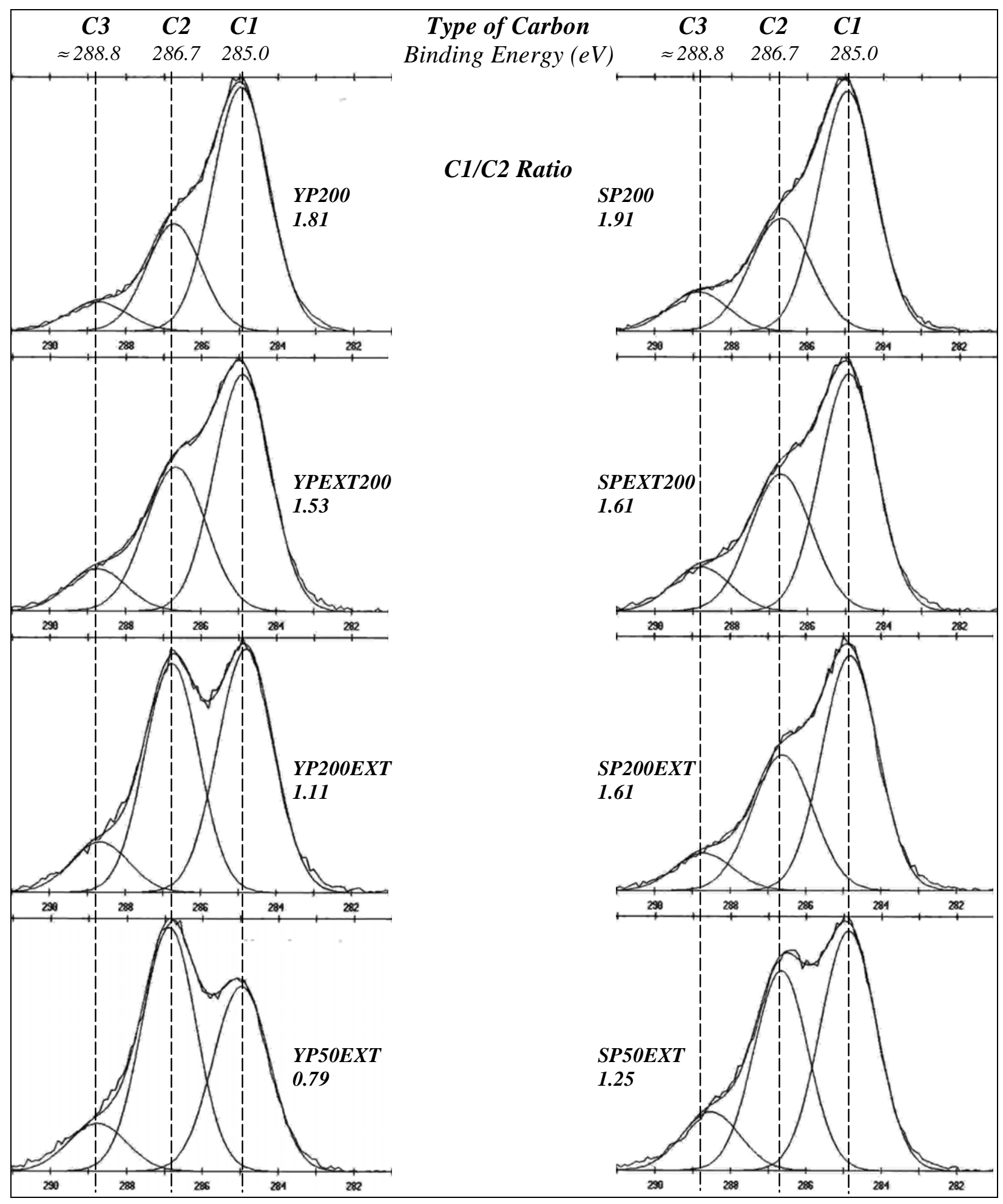

Figure 4.12. Curve fit of Cls peak of XPS spectra for YP (right) and SP (left). 
Since extraction removed most of the extractives (having a high $\mathrm{C} 1 / \mathrm{C} 2$ ratio) from the wood, the decrease of the $\mathrm{C} 1 / \mathrm{C} 2$ ratio after extraction was expected. The assignment of the $\mathrm{C} 1 \mathrm{~s}$ peak to extractives and lignin cannot be distinguished. However, since lignin is relatively immobile, and solvent treatment reduces the $\mathrm{C} 1$ atomic percent, the increased $\mathrm{C} 1 / \mathrm{C} 2$ ratio is likely the result primarily from extractive migration. YP samples exhibited a higher $\mathrm{O} / \mathrm{C}$ ratio and lower $\mathrm{C} 1 / \mathrm{C} 2$ ratio than $\mathrm{SP}$ samples. The difference can be explained with the $\mathrm{O} / \mathrm{C}$ ratio and the $\mathrm{C} 1 / \mathrm{C} 2$ ratio of isolated extractives from YP and SP. Extractives of YP were more CH-type and had a higher $\mathrm{O} / \mathrm{C}$ ratio than $\mathrm{HC}$-type extractives of SP (0.22 and 0.15 , respectively). The $\mathrm{C} 1 / \mathrm{C} 2$ ratio of $\mathrm{YP}$ extractives was lower than the $\mathrm{C} 1 / \mathrm{C} 2$ ratio of SP extractives (3.50 and 6.00, respectively). Therefore, if extractives dominate the wood surface, YP should have a higher O/C and a lower $\mathrm{C} 1 / \mathrm{C} 2$ ratio than SP. The reason for a higher concentration of extractives on SP surfaces is due to the fact that the amount of extractives of the bulk SP is higher $(3.5 \%)$ than of the bulk YP (2.4\%) (Rowe 1989). A type and nature of the extractives is an additional reason for high concentration of extractives on SP. The terpenes and resin acids, which present the main part of SP extractives, are HC-type extractives. They can be divided based on emissions into the condensed $\mathrm{HC}$ with higher molecular weight and the low molecular weight $\mathrm{HC}$, which generally is referred to as volatile $\mathrm{HC}$. The condensed $\mathrm{HC}$ extractives consist mainly of diterpenoids and the volatile part of HC extractives is comprised mainly of monoterpenes (McGraw et al. 1999). At a high temperature, HC-type extractives of SP migrate to the surfaces. The volatile part evaporates, but the condensed part remains at the surface. Further increasing the temperature causes pyrolysis and degradation of extractives, which usually creates acidic products. Both, degraded and unmodified $\mathrm{HC}$ have a low $\mathrm{O} / \mathrm{C}$ ratio and high $\mathrm{C} 1 / \mathrm{C} 2$ ratio, which can explain the low $\mathrm{O} / \mathrm{C}$ and high $\mathrm{C} 1 / \mathrm{C} 2$ ratios of SP when compared with YP.

The binding energies for the $\mathrm{C} 1, \mathrm{C} 2$, and $\mathrm{C} 3$ components of the carbon atom (Figure 4.11) correspond well to the binding energies obtained for carbon atoms of wood material in other studies (Doris and Gray 1978a; Hon 1984; Hua et al. 1993; Kazayawoko et al. 1998). Except for the $\mathrm{C} 3$ component of the cellulose, all three components had similar binding energies. The C3 carbon atom exists either in the carbonyl groups of lignin and extractives, or the C3 occurs as the carbon atom bonded to two oxygen atoms in polysaccharides (Young et al. 1982). 
It seems that the chemical shift due to oxygen in the $\mathrm{C}=\mathrm{O}$ double bound slightly differs from the chemical shift due to two oxygen atoms in the O-C-O bound of cellulose. Istone (1995) reported a small difference in binding energies for the C3 component. Doris and Gray (1978a) found that chemical shift for the C3 component in cellulose was $3.4 \mathrm{eV}$, which meant the C3 component had a binding energy of $288.4 \mathrm{eV}$. The results of this study showed, that the C3 component of cellulose had a slightly smaller binding energy $(288.0 \mathrm{eV})$ than expected.

Besides the C1s curve fit, some studies used curve fit of the O1s peak to interpret the wood surface chemistry. However, the interpretation of the curve fit O1s peak is not as clear as in the case of the $\mathrm{C} 1 \mathrm{~s}$ peak because the O1s peak has a more complex shift behavior (Hua et al. 1993b). The curve fit O1s peak usually comprises three types of oxygen: O1, O2, and O3. Most of the literature (Ahmed et al. 1987; Barry et al. 1990; Kamdem et al. 1991) ascribed the O2 component to cellulose and hemicelluloses, as oxygen in $\mathrm{COH}$ groups, as oxygen in $\mathrm{C}-\mathrm{O}-\mathrm{C}$ link, or as oxygen in 1-4 glycosidic bounds. More contradiction was related to the assignments for O1 and O3 components. Kamdem et al. (1991) ascribed the O1 component to an oxygen atom with a double bound to carbon. The $\mathrm{O} 1$ component could be associated with the lignin and extractives, the elimination of which decreases the O1 fraction (Barry et al. 1990; Hua et al. 1993). However, these studies did not detect the O3 component. Koubaa et al. (1996) attributed O1 and O3 to lignin, and $\mathrm{O} 2$ to hemicelluloses. A clear relationship between the components of $\mathrm{O} 1 \mathrm{~s}$ peak and the wood surface composition cannot be found.

\subsubsection{Wettability of Wood Surfaces}

The results of contact angle measurements on extracted and unextracted wood surfaces are shown in Appendix G and Appendix H, and the average values of the initial contact angle $\left(\theta_{\mathrm{i}}\right)$ are illustrated in (Figure 4.13). Samples of SP exhibited a higher $\theta_{\mathrm{i}}$ than YP for the same surface treatment. The extraction of the samples with acetone-water prior to drying (i.e., YPEXT200 and SPEXT200) did not improve wettability. The extraction, which followed wood drying, improved wettability for both wood species (i.e., YP200EXT and SP200EXT). The lowest $\theta_{\mathrm{i}}$ was obtained on the samples dried at $50^{\circ} \mathrm{C}$ and then extracted. 


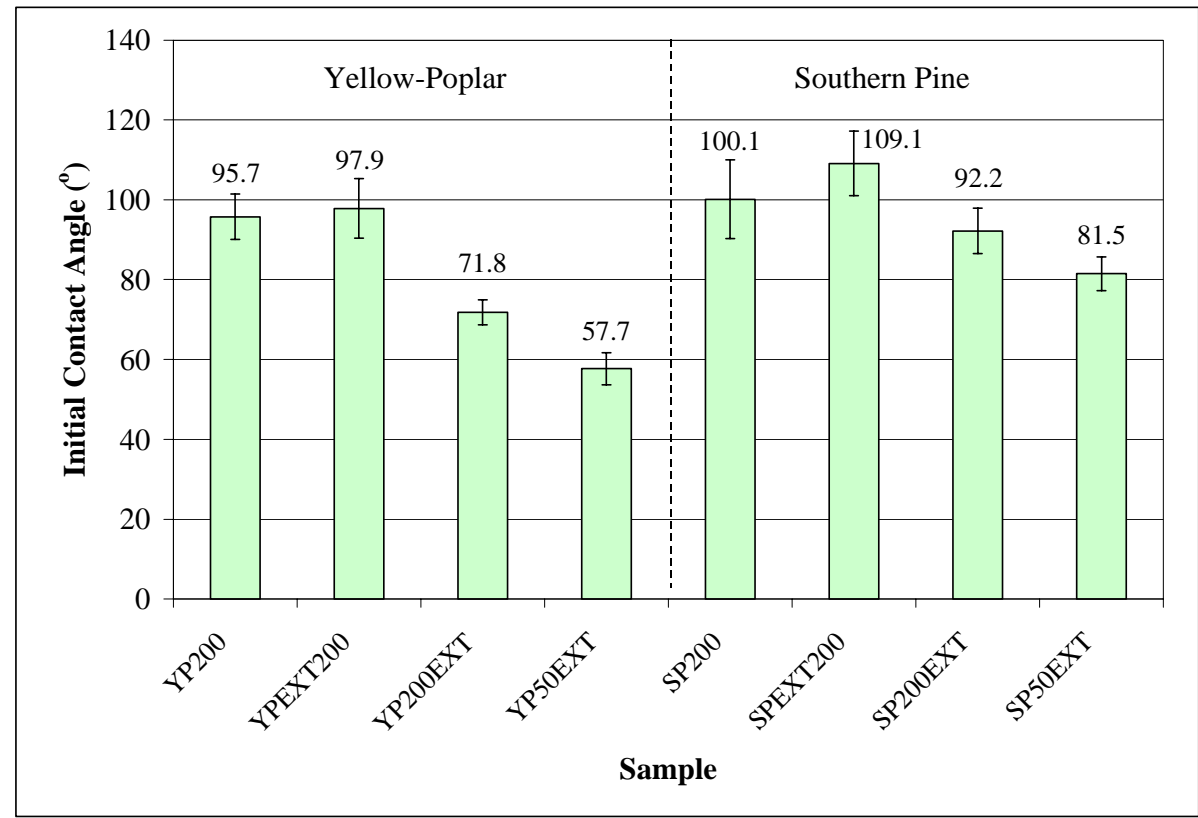

Figure 4.13. Initial water contact angle in regard to wood surface treatment.

The extraction with acetone-water, which followed wood drying, improved wettability for both wood species, because it removed hydrophobic extractives. Thus, the wood surface became more hydrophilic and attractive for water molecules. The extraction of the samples prior to drying (YPEXT200, SPEXT200) did not improve wettability, in fact it increased the contact angle. The increase was significant only for SP. The increase in contact angle of extracted and dried samples could be attributed to the choice of solvent (i.e., acetone-water) that did not remove all types of wood extractives (Hillis 1962). Additionally, VOC emission also occurred from extractive free sample. Less likely but possibly, the increase in the contact angle could be attributed to lignin. When extractives were removed, the hydrophobic lignin remained on the surface. Lignin could even gain ability to intensively concentrate on the wood surface during drying, if acetone was not completely evaporated. One might speculate, that in the presence of acetone during drying, increased mobility of lignin may allow a reorientation at the surface resulting in increased lignin concentration. Besides the effect of acetone and extraction, changes in surface roughness could affect the contact angle (USDA 1999). When the contact angle is greater than $90^{\circ}$, roughness increases this angle still further, but when the contact angle is less than $90^{\circ}$, roughness decreases the angle (Birdi 1997). 
The changes in contact angle during one minute of observation are shown in Figure 4.14 and Figure 4.15. Contact angles decreased with time for both species. The decreasing trend was practically the same for all YP specimens, while the absolute values of contact angle were dependent on surface treatment of YP samples. Contact angle was almost constant during one minute for SPEXT200 specimens. The other SP specimens exhibited a decreasing trend of contact angle during that time. The absolute values of contact angle were dependent on surface treatment of SP samples.

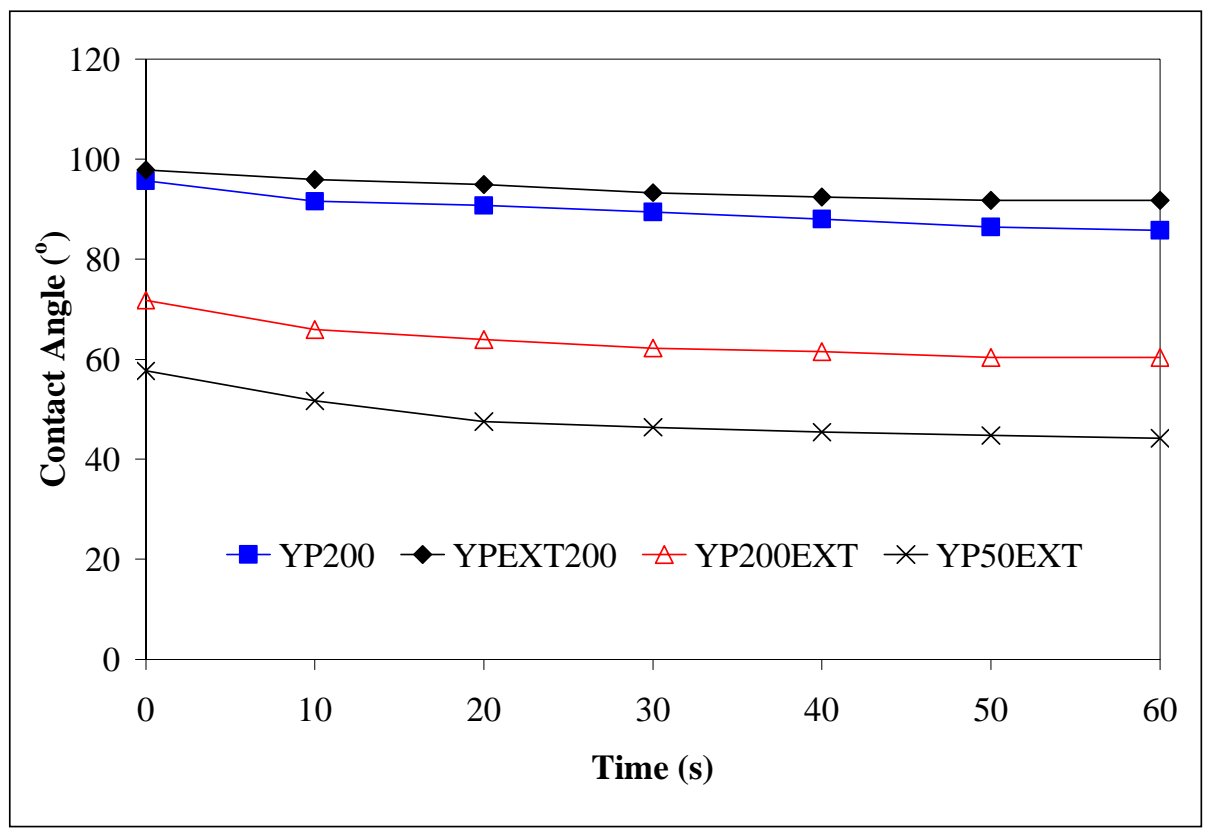

Figure 4.14. Time dependence of contact angle for yellow-poplar.

The water contact angle on the wood surface decreased with time because water was absorbed during the measurement. The absorbtion was higher for samples that were first dried and then extracted because extraction removed hydrophobic extractives and deposited VOCs. Thus, the wood surface became more hydrophilic and attractive for water molecules. Extraction made wood structures also more permeable. It dissolved and removed the deposits that fill cell lumens, or the deposits that were on wood cell walls or attached on pit membranes. 


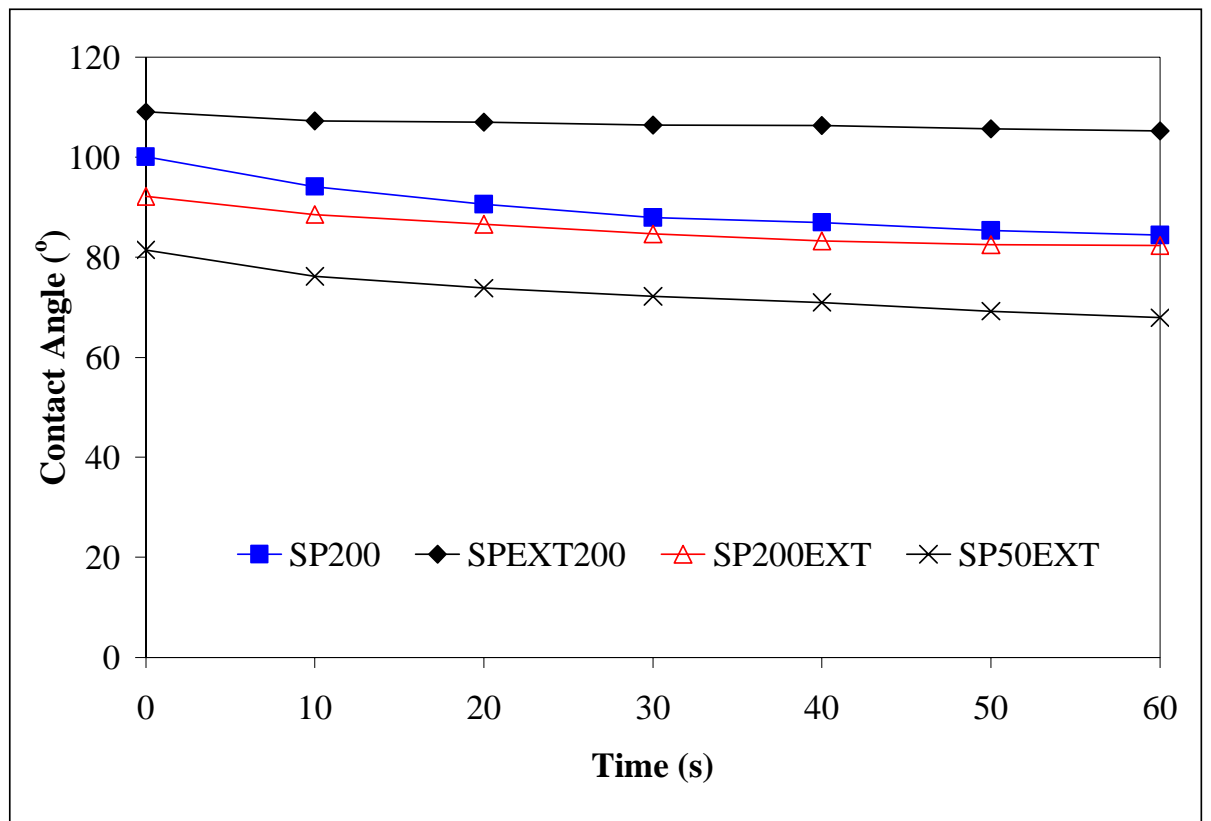

Figure 4.15. Time dependence of contact angle for southern pine.

\subsubsection{Relationship between Wood Surface Chemistry and Wettability}

In spite of some unexpected behavior of the contact angle in regard to the surface treatment, a strong linear relationship was found between wood surface composition and wettability. An initial wettability index (i.e., $\cos \theta_{\mathrm{i}}$ ) was plotted against the $\mathrm{O} / \mathrm{C}$ ratio, and also against the $\mathrm{C} 1 / \mathrm{C} 2$ ratio. Wettability of the wood surface increased with the $\mathrm{O} / \mathrm{C}$ ratio (Figure 4.16) and it decreased with the $\mathrm{C} 1 / \mathrm{C} 2$ ratio (Figure 4.17). In other words, wood wettability decreased with increasing amount of extractives, VOCs, and lignin on the surface. It was shown before (3.4.1) that the amount of extractives increased with decreasing O/C ratio and with increasing $\mathrm{C} 1 / \mathrm{C} 2$ ratio. A linear statistical model explained most of the variability in the initial wettability index data as a function of the $\mathrm{O} / \mathrm{C}$ and the $\mathrm{C} 1 / \mathrm{C} 2$ ratio $-85 \%$ and $91 \%$, respectively. 


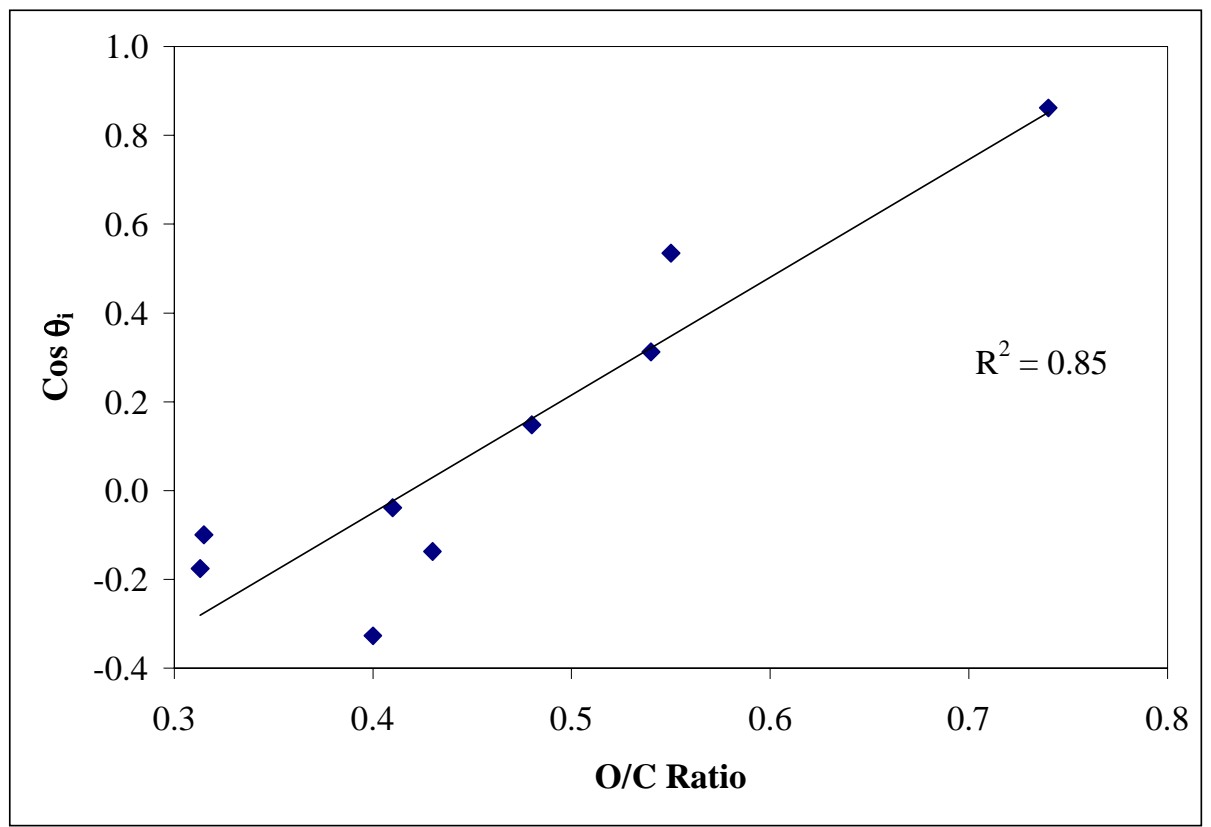

Figure 4.16. Relationship between wettability and the $\mathrm{O} / \mathrm{C}$ ratio of YP and SP surfaces.

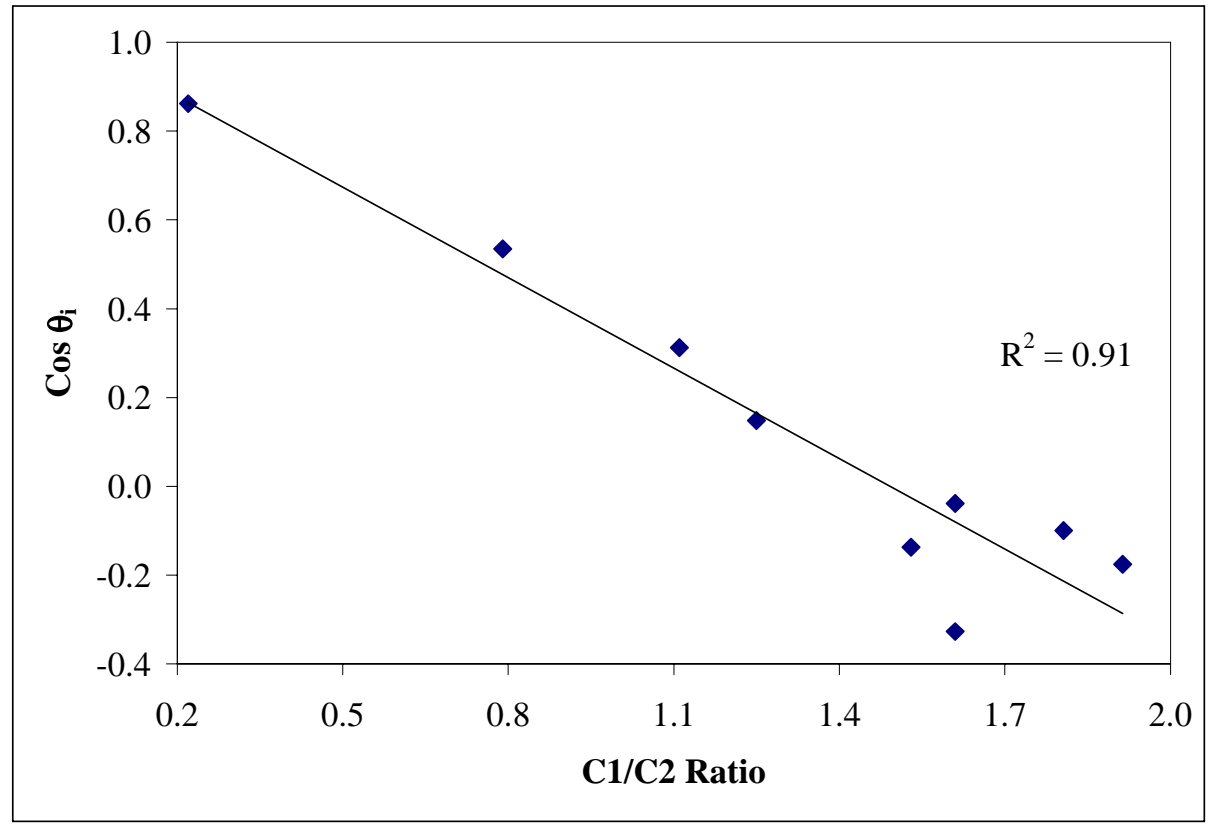

Figure 4.17. Relationship between wettability and the C1/C2 ratio of YP and SP surfaces. 


\subsubsection{Fracture Mechanics}

The strain energy release rate (SERR) was obtained for five samples: SP200, SP200EXT, SPEXT200, YP200, and SPEXT200. The SERR results of PF bonded specimens are shown in Appendix I. The maximum value $\left(\mathrm{G}_{\max }\right)$ and the arrested value $\left(\mathrm{G}_{\mathrm{arr}}\right)$ of the SERR of PF bonded specimens are shown in Figure 4.18.

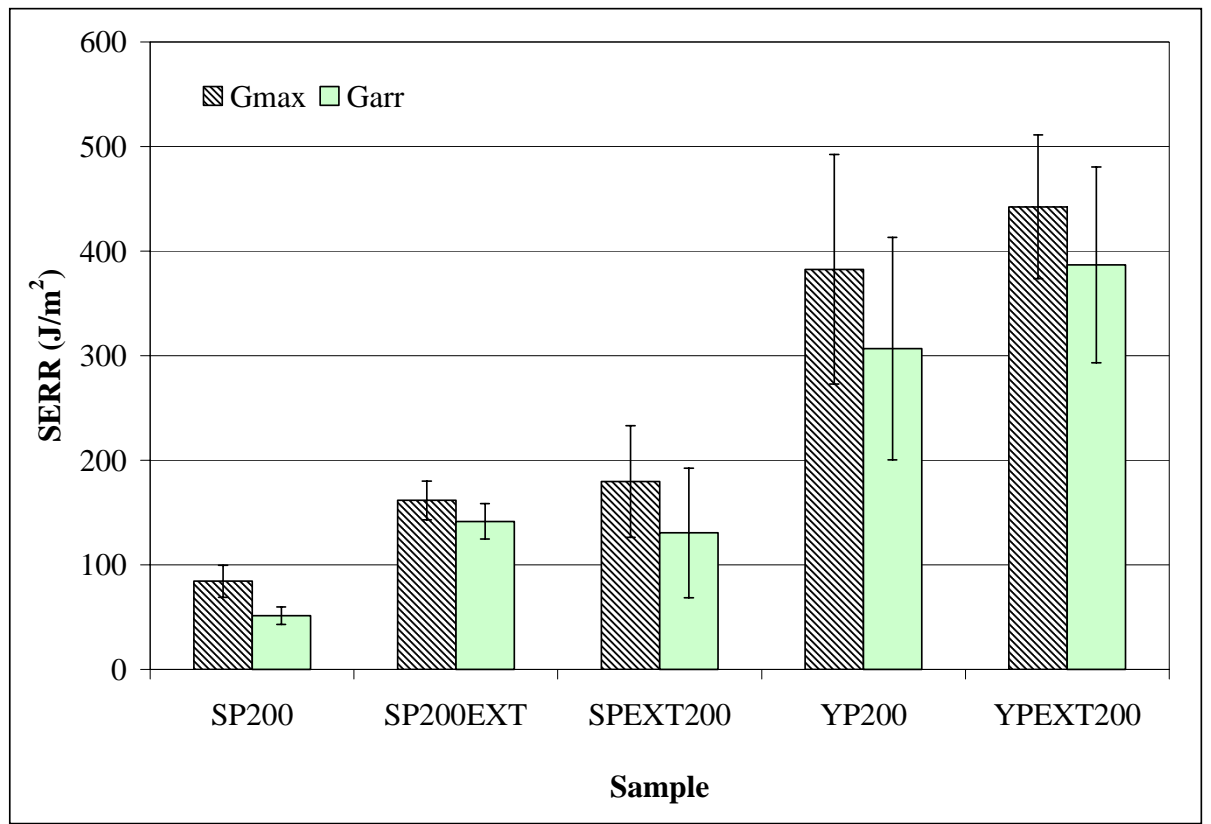

Figure 4.18. Influence of surface treatment on the SERR of PF adhesive bond.

The lowest average SERR of PF adhesive was obtained for SP specimens exposed to a drying temperature of $200^{\circ} \mathrm{C}$ without any extraction. Removal of extractives from wood affected the SERR of samples. Extraction after drying (SP200EXT) or prior to drying (SPEXT200) significantly increased SERR of SP specimens. On the other hand, the removal of extractives from YP wood did not significantly change SERR. The extraction of SP samples doubled the $\mathrm{G}_{\max }$. The increase in SERR was similar for SP200EXT and SPEXT200 samples even though their surface wettability was different (4.4.2). SP200EXT, which exhibited a lower contact angle, possessed a lower $G_{\max }$ and slightly higher $G_{\text {arr }}$ than SPEXT200, which exhibited the highest contact angle. 
The SERR of the YP samples was significantly higher than of the SP samples. The variability of YP specimens was higher than for SP specimens. However, YP surfaces were always better substrates for bonding with PF adhesive than SP surfaces. This was probably due to different amount of extractives between these two wood species. Additionally, YP does not contain HC-type resinous extractives, while SP extractives comprise terpenes and resin acids (Stanley 1969), which are HC-type, non-polar, and very hydrophobic. Therefore, these HC-type extractives are particularly undesirable in terms of adhesion, when bonding wood with waterborne adhesives. Additionally, most SP extractives and degraded product of VOCs are acidic. For example, the major pyrolysis products of pine (Pinus sylvestris) extractives, which were pyrolyzed at temperatures $>400^{\circ} \mathrm{C}$, comprised various aliphatic carboxylic acids and some aliphatic hydrocarbons (Alén et al. 1996).

The results showed that extractives affected adhesion. Their removal resulted in a higher $\mathrm{O} / \mathrm{C}$ ratio, in a lower $\mathrm{C} 1 / \mathrm{C} 2$ ratio, and in a higher SERR. The results on surface chemistry are in agreement with other studies (Kaldas et al. 1998; Mjöberg 1981). On the other hand, some of the wettability results were unexpected. For both wood species, the extraction prior to drying caused an increase in contact angle, which meant decreased wettability. However, a higher adhesion was achieved on these surfaces when compared with unextracted surfaces. It seems that the surface wettability does not correlate with adhesion, if the change of surface properties arises from more than one origin (i.e., high temperature and extraction). In such cases, the mechanism that alters surface properties might change, and an eventual relationship between two surface properties (e.g., wettability and adhesion) collapses. Even though there was no strong relationship between wettability and adhesion of treated specimens, some interesting findings were observed. The SP200EXT and SPEXT200 specimens, which had a similar O/C ratio and the same C1/C2 ratio, had also similar $\mathrm{G}_{\max }$. This was very surprising, if extraction removed most of the extractives, then SPEXT200 specimens were inactivated by some other mechanism when they were exposed to temperature. Pyrolysis might offer an explanation, but this study did not search for evidence to support such an assumption. Reorientation of lignin could also occur. Additionally, it is likely that some extractives remained in wood after extraction. When wood was exposed to high temperature, they migrated to the surfaces and were deposited there on the surface. 


\subsection{Conclusions}

The experimental observation on surface chemistry of wood constituents corresponded to the theoretical interpretation very well. Cellulose had the highest value of the O/C ratio, followed by the O/C ratio of lignin, yellow-poplar extractives, and southern pine extractives. The C1/C2 ratio increased in the following order: cellulose, lignin, YP extractives, and SP extractives.

A high $\mathrm{O} / \mathrm{C}$ ratio or a low $\mathrm{C} 1 / \mathrm{C} 2$ ratio presented a wood surface containing mostly polysaccharides, while a low $\mathrm{O} / \mathrm{C}$ ratio and a high $\mathrm{C} 1 / \mathrm{C} 2$ ratio reflected a high concentration of extractives, VOCs, and lignin on the wood surface. The removal of the extractives increased the $\mathrm{O} / \mathrm{C}$ ratio and decreased the $\mathrm{C} 1 / \mathrm{C} 2$ ratio of the wood surface. The assignment of the carbon $\mathrm{C} 1 \mathrm{~s}$ peak to extractives and lignin cannot be distinguished. However, since lignin is relatively immobile, and solvent treatment reduced the $\mathrm{C} 1$ atomic percent, the increased $\mathrm{C} 1 / \mathrm{C} 2$ ratio was likely the result of extractive migration.

Contact angles observed on the wood surface decreased with time. Southern pine exhibited a higher contact angle than yellow-poplar regardless of the surface treatment. The extraction with acetone-water, which followed wood drying, improved wettability for both wood species. The extraction of the samples prior to drying did not improve wettability, in fact the

contact angle increased. Wettability of the wood surface increased with the O/C ratio and it decreased with the $\mathrm{C} 1 / \mathrm{C} 2$ ratio. 


\section{Chapter 5. Reactivation of Inactivated Wood Surfaces}

\section{$5.1 \quad$ Introduction}

The nature and condition of the adherend surfaces are critical to the success of any bonding (Gauthier 1995), because adhesion, which refers to the attraction between the substances (Kinloch 1987), is a surface phenomenon (Wegman 1989). For a given adhesive and for set curing parameters, the achieved adhesion depends mostly on the surface characteristics. For instance, the surface chemical composition can differ from that of the bulk composition, and the surface may be contaminated by impurities.

Wood surfaces are best prepared for maximum adhesive wetting, flow, and penetration by removing all materials that might interfere with bond formation to wood (USDA 1999). A fresh surface contains all of the molecular attractive forces that previously held the material together (Marra 1992). Thus, a fresh surface assures the highest adhesion. When the attractive forces on the surface are reduced_-inactivated wood surface-adhesion is diminished and weak.

A satisfactory remedy for thermal inactivation in the wood-based composites industry has not been found. However, adhesion between inactivated wood surfaces may be improved by several means. An adhesive, which can penetrate through the inactivated layer, and which has a high affinity for attraction with the substrate, should amplify adhesive bond performance. Besides this, surface cleaning, surface removal, and surface treatment improve the adhesion between inactivated surfaces. However, surface treatment prior to bonding is not a desirable process. Any surface treatment presents additional cost, often requires special dispensing equipment, and demands extra time. Additionally, application of a surface treatment is often limited by characteristics of the adherend (e.g., size, shape, and surface irregularity), or hindered by process requirements (e.g., vacuum, emissions, and speed). In the past decade, some novel bonding techniques (Haupt and Sellers 1994; Pizzi 1994; Rowell 1995) were developed and different surface treatment methods were used to improve adhesion. A partial improvement of adhesion was achieved when inactivated wood surfaces were chemically treated (Chow 1975; Christiansen 1990). Lu et al. (2000) reviewed coupling agents and chemical treatments in areas of wood fiber and polymer composites. 
The coupling agent interacts with the surface and increases its surface tension. This improves surface wettability, which often leads to a stronger adhesive bond. Some promising results have been achieved with a new coupling agent hydroxymethylated resorcinol (Vick et al. 1996). Additionally, bio-products, such as enzymes, or other chemicals, such as sodium hydroxide $(\mathrm{NaOH})$, can be included in the surface preparation to improve wood surface properties in terms of wettability and adhesion

\subsubsection{Hydroxymethylated Resorcinol}

Hydroxymethylated resorcinol (HMR) is an effective a coupling agent for wood. A coupling agent is a molecule with different or like functional groups, which is capable of reacting with surface molecules of two different substances, thereby chemically bridging the substances (Vick 1995). Surface treatment with HMR enhances adhesion to wood for epoxy, phenolformaldehyde, phenol-resorcinol-formaldehyde, polymeric/isocyanate, melamine-formaldehyde, urea-formaldehyde, and melamine-urea-formaldehyde adhesive (Vick et al. 1998).

After HMR treatment, a wood surface becomes enriched with functional hydroxymethyl groups. A proposed mechanism of adhesion improvement is attributed to the coupling action of HMR, where ether linkages are formed between hydroxymethyl groups of the HMR (Figure 5.1) and the primary alcohols (hydroxyls) of both the wood and thermosetting adhesives (Gardner $e t$ al. 2000). The resulting covalent bonds are hydrolytically stable, thus providing durability of the adhesive bond. However, the increased durability of HMR-treated adhesive bonds is attributed to either covalent bonding or to high-density hydrogen bonding (Vick and Okkonen 2000).

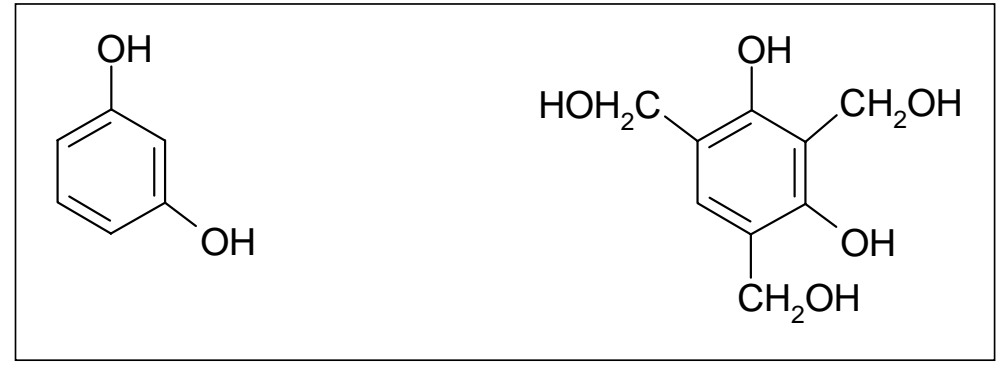

Figure 5.1. Resorcinol (left) and trihydroxymethyl resorcinol (right). 


\subsubsection{Xylanases}

Enzymes are often used in the paper industry to improve pulp yield and fiber properties. Cellulase enzymes can increase the relative bonded area of the fibrous paper network (Garcia $e t$ al. 2001). Xylanase can selectively solubilize xylan (Schönberg et al. 2001). These enzymes can be used for the conversion of hemicelluloses by agricultural and food industries.

Xylanases (1,4- $\beta$-D-xylan xylanohydrolase) have raised considerable interest in the past decade, especially for their application in the bleaching process in the pulp and paper industry (Jeffries and Viikari 1996). A group of xylanase enzymes can remove the lignin from wood fibers, which occurs through breaking the hemicellulose chains that are responsible for the adherence of lignin to the cellulose (Davis et al. 1997). Breaking of hemicellulose chains might provide some new bonding sides for adhesive. Surface modification by xylanase might lead to improved adhesion if the cause of inactivation arises from hornification of wood fibers.

\subsubsection{Sodium Hydroxide}

Wood surfaces treated with $\mathrm{NaOH}$ exhibited enhanced wettability and partially improved adhesion (Christiansen 1990). $\mathrm{NaOH}$ has at least two beneficial effects on wettability and bonding. First, a treatment of a wood surface with a low concentration of $\mathrm{NaOH}$ aqueous solutions increases surface free energy and thus, improves surface wettability. The application of a $10 \%$ sodium hydroxide solution to a wood surface helped restore wood surface bondability to a certain degree, especially at longer assembly times (Christiansen 1990). Second, alkali $\mathrm{NaOH}$ neutralizes an acidic wood surface, which results in a more adequate cure reaction of alkali PF adhesive. Otherwise, acid extractives often prolong the curing of PF adhesives (Hse and Kuo 1988; Wellons 1977).

\subsubsection{Objectives}

The objectives of this study were to reactivate the inactivated wood surface by a chemical treatment to enhance the wettability and to improve adhesive bond performance. The effect of different adhesive mixtures on adhesion was also studied. 


\subsection{Materials Preparation}

This experiment used only southern pine (SP), since this wood species showed severe surface inactivation. Heartwood of SP, with green moisture content, was cut into tangential lamellas $(120 \times 320 \mathrm{~mm})$ and planed to a thickness of 12 or $10 \mathrm{~mm}$. Wood samples were sorted in two groups (control and inactivated) and then separately dried in a convection oven to $2 \% \mathrm{MC}$. The control group (SPC), in which 4 lamellas were $12 \mathrm{~mm}$ thick, was dried at $50^{\circ} \mathrm{C}$. The inactivated group (SPI), in which 20 lamellas were $10 \mathrm{~mm}$ thick, was dried at $200^{\circ} \mathrm{C}$. Wood drying was carried out using the parameters listed in Table 5.1.

Table 5.1. Properties of Wood Samples and Drying Parameters.

\begin{tabular}{|c|c|c|}
\hline \multirow{2}{*}{$\begin{array}{l}\text { Wood Specification and } \\
\text { Drying Parameters }\end{array}$} & \multicolumn{2}{|c|}{ Set Point Temperature $\left({ }^{0} \mathrm{C}\right)$} \\
\hline & 50 & 200 \\
\hline Number of lamellas & 4 & 20 \\
\hline Initial Average $\mathrm{MC}(\%)$ & 28.9 & 98.0 \\
\hline Final Average MC (\%) & $2-4$ & $<2$ \\
\hline Max. Surface Temp. $\left({ }^{0} \mathrm{C}\right)$ & 51 & 191 \\
\hline Drying Time (hrs:min) & $24: 00$ & $6: 20$ \\
\hline
\end{tabular}

\subsubsection{Drying of Wood Samples}

SPC and SPI lamellas were dried separately. The actual MC was controlled by the weight measurement of the samples during drying. A computer monitored the temperature of the wood surface of one SPI lamella every minute, while the temperature of the drying air was recorded manually every 10 minutes (Figure 5.2). This information was needed for keeping the samples for a certain time above the temperature level $\left(>150^{\circ} \mathrm{C}\right)$ that caused surface inactivation. For SPC, only a maximum surface temperature was controlled, since the surface of this sample was removed after drying. Thus, any measurement of the changes in temperature during drying was irrelevant. After drying, the samples were cooled to room temperature. Dried lamellas were then stored for two days prior to surface treatment. 


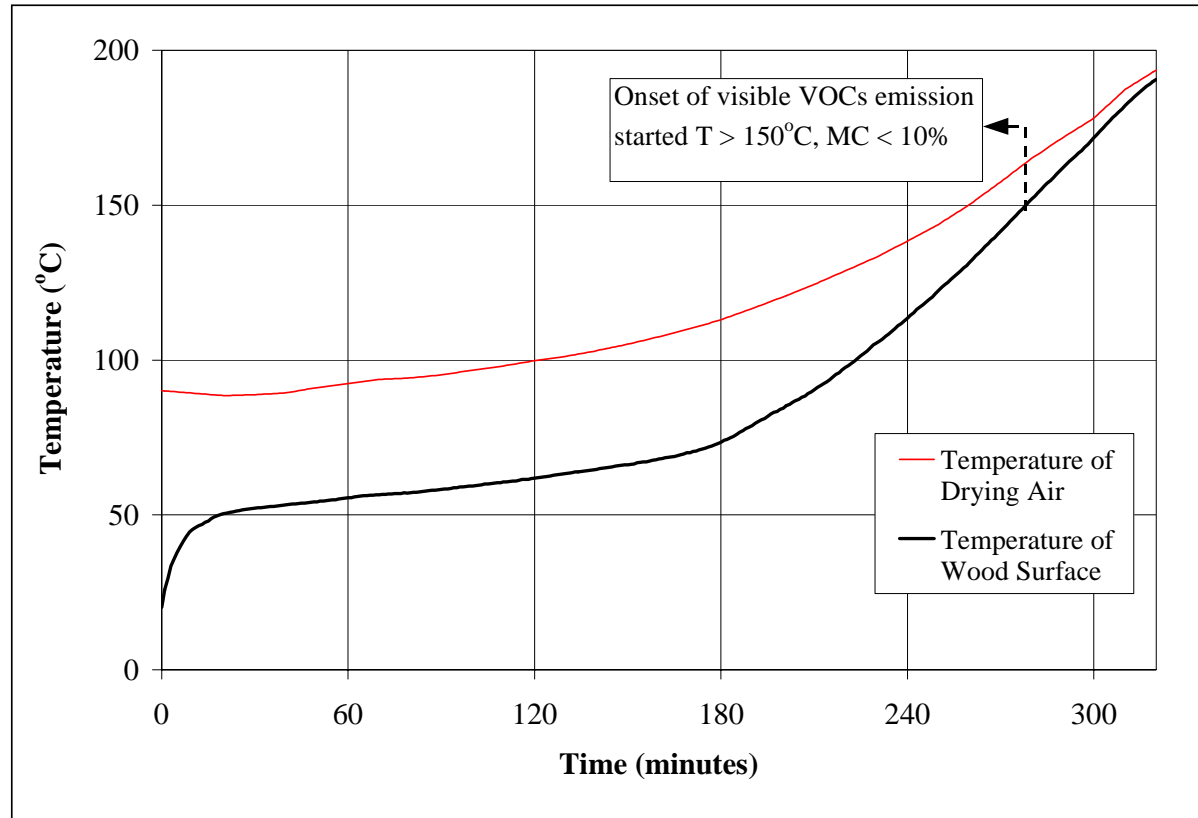

Figure 5.2. Changes in temperatures during wood drying - a typical plot.

\subsubsection{Surface Treatment}

After drying, four lamellas of the inactivated sample were stored and their surfaces remained untouched. All other inactivated lamellas were treated with aqueous solutions of several chemicals. A sponge was used to manually apply the chemical solution on the surface at a spreading level of $100 \mathrm{~g} / \mathrm{m}^{2}$. The control sample was not treated, but its surface was removed. In total, six different samples were prepared (Table 5.2).

Table 5.2. Treatment of the samples for surface reactivation.

\begin{tabular}{lllll}
\hline Sample & & \multicolumn{1}{c}{ Surface treatment } & & \multicolumn{1}{c}{ Description/Specification } \\
\cline { 1 - 1 } SPC & & Surface removed by planing & & Control sample dried at $50^{\circ} \mathrm{C}$ \\
SPI & & Inactivated surface without surface removal & & Inactivated sample dried at $200^{\circ} \mathrm{C}$ \\
SPIHMR & & SPI treated with hydroxymethylated resorcinol & & Vick et al. 1996 , US Patent $5,543,487$ \\
SPIXY & & SPI treated with xylanase & & $10 \%$ aqueous solution of xylanase \\
SPINA & & SPI treated with sodium hydroxide $(\mathrm{NaOH})$ & & $4 \%$ aqueous solution of $\mathrm{NaOH}$ \\
SPIXYNA & SPI treated with xylanase and sodium hydroxide & & SPIXY washed with $\mathrm{NaOH}$ and water \\
\hline
\end{tabular}




\subsubsection{Control Sample}

The control sample (SPC) was dried in a convection oven at $50^{\circ} \mathrm{C}$ for 24 hours and then its surface was removed by planing prior to analysis. This sample was $2 \mathrm{~mm}$ thicker than the other samples in order to achieve the same final thickness (i.e., $10 \mathrm{~mm}$ ). Since planing opened a fresh wood surface, this sample should provide maximum adhesion forces.

\subsubsection{Inactivated Sample}

The inactivated sample (SPI) was dried in a convection oven at $200^{\circ} \mathrm{C}$ for 6 hours and 20 minutes. Maximum achieved surface temperature was $190.6^{\circ} \mathrm{C}$. The surface was not removed or treated with any chemicals. This sample should have the lowest adhesive bond performance. Besides four lamellas within this sample, 16 additional lamellas were prepared by the same drying procedure for further chemical treatment of the surface.

\subsubsection{Inactivated Sample Treated with Hydroxymethylated Resorcinol}

Four SPI lamellas were treated with an HMR coupling agent. HMR was prepared according to the patented procedure with the composition given in Table 5.3 (Vick et al. 1996).

Table 5.3. Ingredients for the HMR coupling agent.

\begin{tabular}{llc}
\hline Ingredients & & Parts by Weight \\
\cline { 1 - 1 } Water, deionized & & 90.43 \\
Resorcinol, crystalline & 3.34 \\
Formaldehyde, 37\% & 3.79 \\
Sodium hydroxide, 3M & & 2.44 \\
\hline Total & & 100.00 \\
\hline
\end{tabular}

HMR reacted at a temperature of $23^{\circ} \mathrm{C}$ for 6 hours, which was identified to be an optimum time providing best durability performance (Vick et al. 1998). The color of the HMR solution was transparent initially, and then turned to pink, brown, and dark brown within the first five minutes. At the end of the reaction time, the HMR solution color was dark brown-red. 
The initial $\mathrm{pH}$ value of the HMR solution was 11 and it increased to 13 at the end of the reaction. The HMR solution was then applied to the wood surface, which color turned from yellow to brown-red. Drying at $50^{\circ} \mathrm{C}$ evaporated the excess water from the surface. The lamellas were then stored in a plastic bag. HMR treated surfaces (SPIHMR) were analyzed and bonded 24 hours after the treatment.

\subsubsection{Inactivated Sample Treated with Enzyme Xylanase}

Four SPI lamellas were treated with a solution of enzymes. Sigma Aldrich ${ }^{\circledR}$ supplied enzymes xylanase Pentopan Mono BG ${ }^{\mathrm{TM}}$. Xylanase were prepared as $1 \%$ aqueous solution. This concentration was within the concentration range used in other studies (Davis et al. 1997; Schönberg et al. 2001). The reaction time was 30 minutes at $45( \pm 2)^{\circ} \mathrm{C}$, which was within the temperature range that provided the highest xylanase activity (Gupta et al. 2000).

The initial $\mathrm{pH}$ value of the solution was 4.1 and it increased to 5 at the end of the reaction time. Xylanase solution was then applied to the wood surface, which color turned from yellow to bright yellow. Drying at $50^{\circ} \mathrm{C}$ evaporated the excess water from the surface. The lamellas were then stored in a plastic bag. Xylanase treated surfaces (SPIXY) were analyzed and bonded 24 hours after the treatment.

\subsubsection{Inactivated Sample Treated with Sodium Hydroxide}

Four SPI lamellas were treated with $4 \%$ aqueous solution of $\mathrm{NaOH}$. The solution had a $\mathrm{pH}$ of 14 . When this solution was applied to the wood surface, the color of the surface turned from yellow to yellow-brown. Drying at $50^{\circ} \mathrm{C}$ evaporated the excess water from the surface. The lamellas were then stored in a plastic bag. $\mathrm{NaOH}$ treated surfaces (SPINA) were analyzed and bonded 24 hours after the treatment. 


\subsubsection{Inactivated Sample Treated with Xylanase and Sodium Hydroxide}

Four SPI lamellas were prepared under the same procedure used for SPIXY. The treated surfaces were then washed with a $4 \%$ aqueous solution of $\mathrm{NaOH}$ by using a sponge. $\mathrm{NaOH}$ stayed on the surface for 10 minutes and then it was washed with distilled water. After washing the surface color was yellow. Drying at $50^{\circ} \mathrm{C}$ evaporated the excess water from the surface. The lamellas were then stored in a plastic bag. The SPIXYNA specimens were analyzed and bonded 24 hours after washing with water.

\subsubsection{Specimen Cutting}

After surface treatment and evaporation of excess water, each lamella was cut into individual specimens for different study purposes (Figure 5.3).

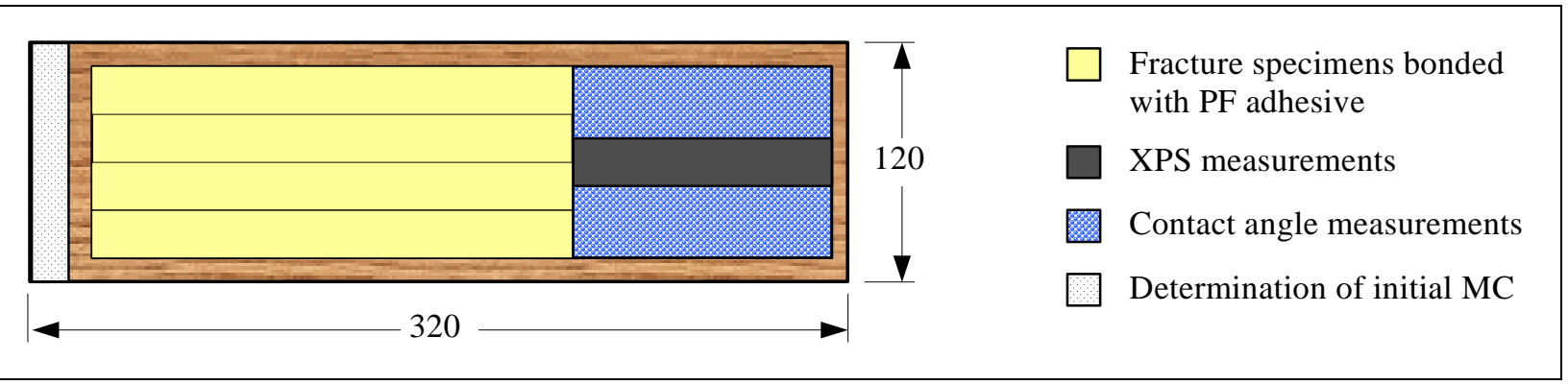

Figure 5.3. Specimen cutting diagram for each lamella. Width ( $\mathrm{mm})$ is tangential direction.

\subsubsection{Adhesives}

Four adhesive mixtures were used for the evaluation of the adhesive bond performance of treated wood surfaces: PF, PVA, PFHMR, and PMDI. The Dynea supplied the PF adhesive, National Starch and Chemical supplied the PVA adhesive, and the Dow Chemical Company supplied the PMDI adhesive. PF adhesive was used with all surface treatments. PVA adhesive was used with all except the HMR treatment. PF adhesive, which was mixed together with HMR (PFHMR), and PMDI adhesive were used only with inactivated wood surfaces. PFHMR comprised 5 parts of PF adhesive and one part of HMR, which was prepared as explained in 5.2.2.3. The PFHMR mixture was prepared 5 minutes prior to the adhesive application. 
All adhesives were stored approximately for one month prior to bonding, except the PMDI adhesive. This adhesive was 15 months old. The decision of using PMDI adhesive was made subsequently, since there remained several inactivated SP lamellas after bonding with other adhesive mixtures. There was no particular reason to use an old adhesive, but such PMDI adhesive was available at the time of bonding and the self-life is very long. Table 5.4 provides specifications and properties of the adhesive mixtures. The measurements were obtained according to the standard procedures (ASTM 1997). The adhesive viscosity was monitored with a Brookfield viscometer for 20 minutes at $20^{\circ} \mathrm{C}$.

Table 5.4. Specifications of the adhesive mixtures and curing parameters.

\begin{tabular}{|c|c|c|c|c|}
\hline \multirow{3}{*}{$\begin{array}{l}\text { Adhesive } \\
\text { Property }\end{array}$} & \multicolumn{4}{|c|}{ Adhesive Mixture and Name } \\
\hline & PF & PVA & PFHMR & PMDI \\
\hline & $\begin{array}{c}\text { Chembond }{ }^{\circledR} \\
\text { CB } 303\end{array}$ & $\begin{array}{c}\text { KOR LOK® } \\
\text { GT 42-300 }\end{array}$ & $\begin{array}{c}\text { Chembond }{ }^{\circledR} \text { CB } \\
303 \text { + HMR }\end{array}$ & $\begin{array}{c}\text { ISOBIND }{ }^{\circledR} \\
1088\end{array}$ \\
\hline Physical state & Liquid & Liquid & Liquid & Liquid \\
\hline Solids content $(\%)$ & 45.8 & 51.5 & 37.1 & 100.0 \\
\hline $\mathrm{pH}$ value & 11 & 3.5 & 12 & Not applicable \\
\hline Viscosity (cps) & 1580 & 32500 & 62 & 220 \\
\hline Application rate $\left(\mathrm{g} / \mathrm{m}^{2}\right)$ & 200 & 200 & 200 & 200 \\
\hline Cure temperature $\left({ }^{\circ} \mathrm{C}\right)$ & 200 & 20 & 200 & 200 \\
\hline Pressure $\left(\mathrm{N} / \mathrm{mm}^{2}\right)$ & 2.0 & 2.0 & 2.0 & 2.0 \\
\hline Cure time & $15 \mathrm{~min}$ & $60 \mathrm{~min}$ & $15 \mathrm{~min}$ & $15 \mathrm{~min}$ \\
\hline
\end{tabular}

\subsection{Methods}

\subsubsection{X-Ray Photoelectron Spectroscopy}

A Perkin-Elmer model $5400 \mathrm{X}$-ray photoelectron spectrometer was employed to provide elemental and chemical data of the treated wood surfaces. The measurements were performed as described in section 3.3.1. Three replicate measurements per surface treatment provided 18 XPS spectra results in total. 


\subsubsection{Contact Angle Measurement}

A sessile drop method was used to measure the contact angle $(\theta)$ of a $5 \mu$ l drop of several liquids. All measurements were performed as described in section 3.3.2. First, a drop of distilled water was applied to the wood surface and $\theta$ was measured for all surface treatments. Second, a contact angle of PF adhesive drop was evaluated on the same wood surfaces. In both cases, the image was captured immediately after the drop was applied ( 0 seconds), and then every 10 seconds for a duration of one minute. Last, an initial contact angle of liquid probes with different surface tension (Table 5.5) was obtained. These data were needed for the evaluation of the critical surface tension of the treated wood surfaces as described by Zisman (1964). All measurements were carried out at $20( \pm 1)^{\circ} \mathrm{C}$.

Table 5.5. Surface tension of liquid probes.

\begin{tabular}{lcc}
\hline Liquid probe & & Surface tension $(\mathbf{m N} / \mathbf{m})$ \\
\cline { 1 - 1 } Water & 72.8 \\
Glycerol & 64.0 \\
Formamide & 58.3 \\
Ethylene glycol & 48.3 \\
Bromonaphthalene & 44.6 \\
\hline
\end{tabular}

\subsubsection{Fracture Mechanics Test}

The test specimens were prepared as described in 3.3.3, with some modifications in dimensions (Figure 5.4). One sample comprised 8 fracture specimens. In total, 104 specimens were tested according to the defined procedure in 3.3.3.

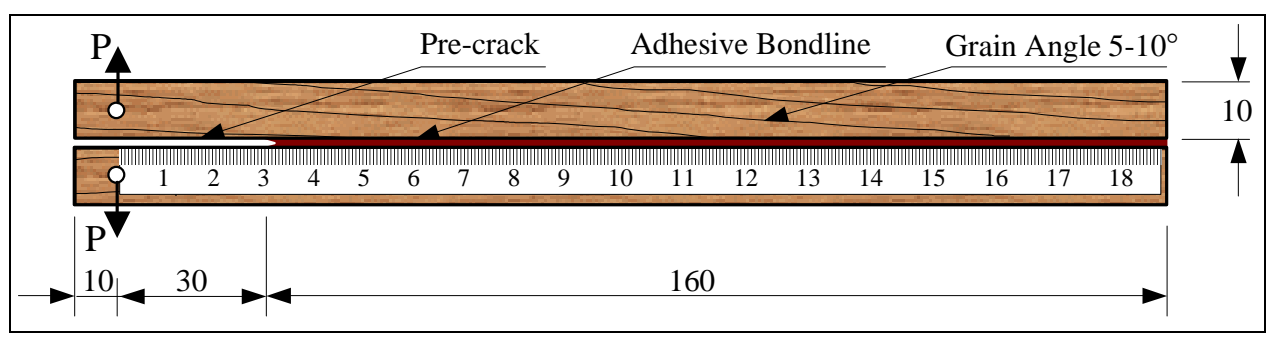

Figure 5.4. Orientation, geometry and dimensions $(\mathrm{mm})$ of the fracture test specimen. 


\subsection{Results and Discussion}

\subsubsection{Chemistry of Treated Wood Surfaces}

Surface chemical composition of the SP sample changed after the treatments. Atomic percent of treated southern pine samples is shown in Table 5.6.

Table 5.6. Atomic percent of treated southern pine surfaces.

\begin{tabular}{|c|c|c|c|c|}
\hline \multirow{3}{*}{$\begin{array}{c}\text { Surface } \\
\text { Treatment }\end{array}$} & \multicolumn{4}{|c|}{ Atomic Percent } \\
\hline & \multicolumn{4}{|c|}{ Elements } \\
\hline & $\mathrm{C}$ & $\mathbf{O}$ & $\mathbf{N}$ & $\mathbf{N a}$ \\
\hline SPC & 84.4 & 15.4 & 0.2 & 1 \\
\hline SPI & 85.1 & 14.3 & 0.6 & I \\
\hline SPIHMR & 82.6 & 17.4 & I & I \\
\hline SPIXY & 81.3 & 16.0 & 2.7 & I \\
\hline SPINA & 79.5 & 18.4 & 0.6 & 1.5 \\
\hline SPIXYNA & 76.4 & 20.9 & 1.2 & 1.5 \\
\hline
\end{tabular}

All treatments of the SPI sample resulted in decreased carbon content and increased oxygen content. A small, but not significant, amount of nitrogen and sodium was detected for some treatments. It was expected to obtain differences in atomic percent among samples, since the treatments used chemically different substances, which remained at the wood surface after water evaporation. Detailed results of XPS analysis and the calculated O/C and C1/C2 ratios are shown in Table 5.7.

Table 5.7. Elemental components of southern pine surface as determined by XPS.

\begin{tabular}{|c|c|c|c|c|c|c|c|c|c|}
\hline \multirow{3}{*}{$\begin{array}{c}\text { Surface } \\
\text { Treatment }\end{array}$} & \multicolumn{7}{|c|}{ Atomic Percent } & \multirow{2}{*}{\multicolumn{2}{|c|}{$\begin{array}{c}\text { Atomic } \\
\text { Ratio }\end{array}$}} \\
\hline & \multicolumn{4}{|c|}{ C1s Components } & \multicolumn{3}{|c|}{ O1s Components } & & \\
\hline & C1 & $\mathrm{C2}$ & C3 & $\mathrm{C4}$ & 01 & $\mathrm{O2}$ & $\mathbf{O 3}$ & $\mathrm{O} / \mathrm{C}$ & $\mathrm{C} 1 / \mathrm{C} 2$ \\
\hline SPC & 74.8 & 20.4 & 4.4 & 0.9 & 10.6 & 77.3 & 12.1 & 0.18 & 3.68 \\
\hline SPI & 75.2 & 17.2 & 6.1 & 1.5 & 17.1 & 59.6 & 23.3 & 0.17 & 4.38 \\
\hline SPIHMR & 71.2 & 18.9 & 6.7 & 3.2 & 26.8 & 55.8 & 17.4 & 0.21 & 3.81 \\
\hline SPIXY & 72.6 & 19.0 & 8.5 & 0.0 & 19.8 & 58.4 & 21.9 & 0.20 & 3.88 \\
\hline SPINA & 68.3 & 21.8 & 6.9 & 3.1 & 22.2 & 55.5 & 22.4 & 0.23 & 3.15 \\
\hline SPIXYNA & 66.2 & 23.4 & 7.2 & 3.1 & 19.8 & 57.5 & 22.8 & 0.27 & 2.83 \\
\hline
\end{tabular}


The $\mathrm{O} / \mathrm{C}$ ratio of chemically treated surfaces was higher, and the $\mathrm{C} 1 / \mathrm{C} 2$ ratio was lower, than of the inactivated SPI surface. Surprisingly a low O/C ratio and a high $\mathrm{C} 1 / \mathrm{C} 2$ ratio were obtained for the control SPC sample. Since only one or two measurements were done for each surface treatment, it might happen that an area of SPC containing a resin canal was analyzed. Extractives content is higher in latewood than in earlywood, and is enormous in resin canals. Therefore, a fresh surface might exhibit lower $\mathrm{O} / \mathrm{C}$ and higher $\mathrm{C} 1 / \mathrm{C} 2$ ratios than expected because of the variability and heterogeneity of wood. A surface contamination of the SPC sample might also be reason for a higher $\mathrm{O} / \mathrm{C}$ ratio than expected.

\subsubsection{Effect of Surface Treatment on Wettability of Southern Pine}

The results of contact angle measurements on extracted and unextracted wood surfaces are shown in Appendix $J$ and Appendix K. An average value of the initial contact angle $\left(\theta_{i}\right)$ of a water drop on a fresh wood surface (SPC) was $45.7^{\circ}$, which was consistent with the magnitude of the contact angle measured on unmodified wood in other studies (Kajita and Skaar 1992; Liptakova et al. 1995). Figure 5.5 shows the influence of surface treatment on $\theta_{\mathrm{i}}$.

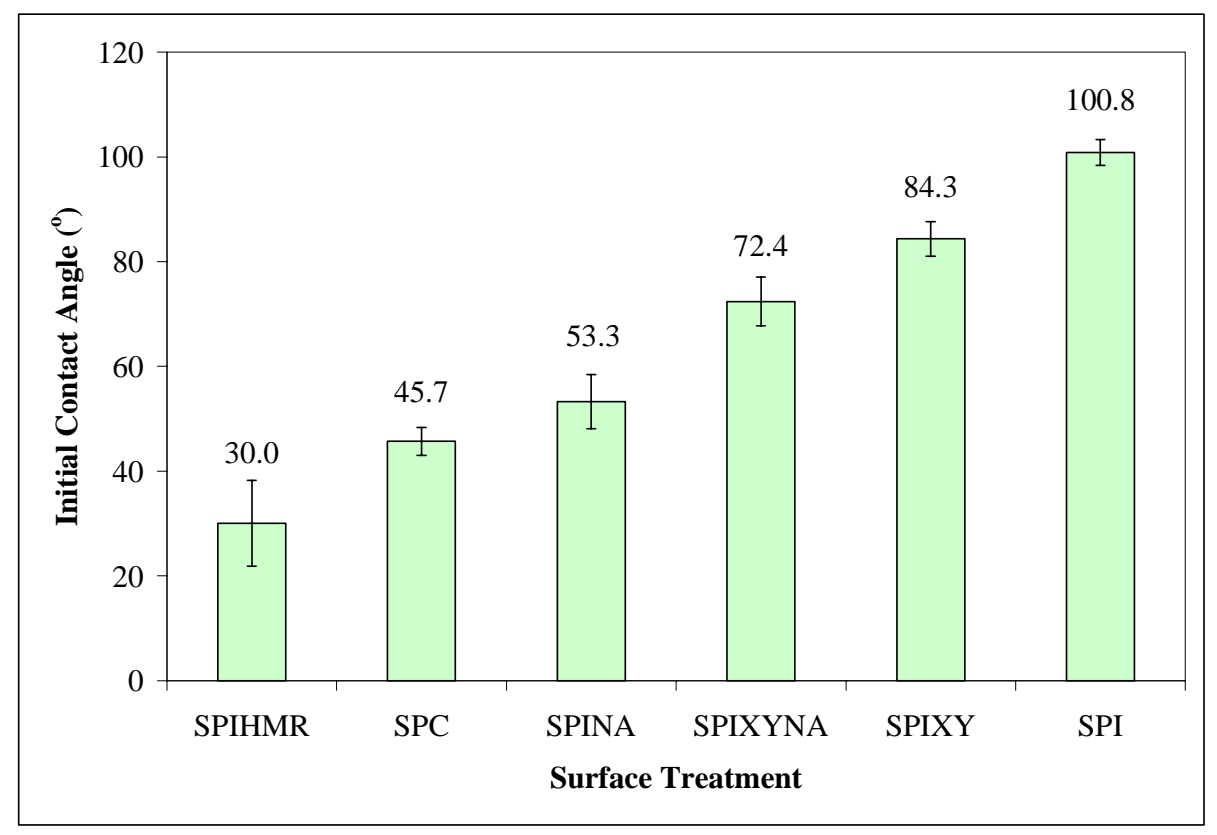

Figure 5.5. Influence of surface treatment on initial water contact angle. 
Chemical treatments of the wood surface strongly influenced wettability. The highest $\theta_{\mathrm{i}}$ was obtained on the inactivated wood surface (SPI), and the lowest $\theta_{\mathrm{i}}$ occurred on the SPIHMR sample, which was treated with hydroxymethylated resorcinol (HMR) All the chemical treatments containing $\mathrm{NaOH}$ (i.e., SPIHMR, SPINA, and SPIXYNA) improved the water wettability of inactivated wood surfaces. $\mathrm{NaOH}$ increased the surface free energy of wood, which is preferential for adhesive spreading and wetting as shown by Equation 2.2. Therefore, these results of water contact angle were expected, but a clear relationship between $\theta_{\mathrm{i}}$ and the $\mathrm{O} / \mathrm{C}$ or the $\mathrm{C} 1 / \mathrm{C} 2$ ratio was not found. This was probably because the causes that change surface properties arise from more than one origin. For instance, SPC surface was not treated either thermally or chemically, SPI was treated only thermally, and the other four samples were treated thermally and with different chemicals. Regardless of surface treatment, the water contact angle decreased with time for all samples, which is shown in Figure 5.6.

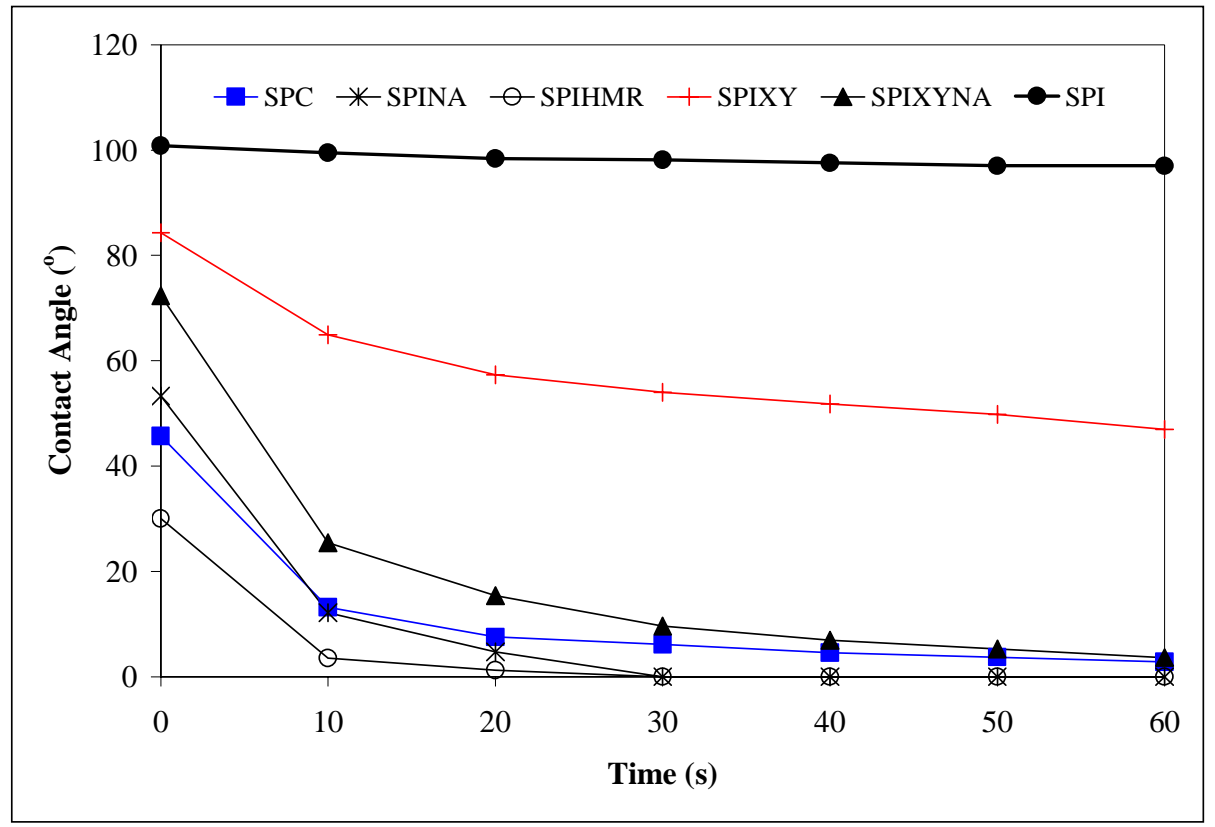

Figure 5.6. Influence of time and surface treatment on the contact angle of a water drop. 
The decline of the contact angle during one minute of observation was insignificant $\left(4^{\circ}\right)$ for the inactivated wood surfaces of the SPI sample. For all other surfaces, contact angle declined significantly during one minute. The highest change in the contact angle was obtained on SPIXYNA specimens $\left(69^{\circ}\right)$, followed by SPINA $\left(53^{\circ}\right), \operatorname{SPC}\left(43^{\circ}\right), \operatorname{SPIXY}\left(37^{\circ}\right)$ and $\operatorname{SPIHMR}\left(30^{\circ}\right)$.

Gardner et al. (2000) reported that $\theta_{\mathrm{i}}$ of PF resin on HMR-treated southern pine was higher $\left(87.5^{\circ}\right)$ when compared with an untreated surface $\left(77.2^{\circ}\right)$. A similar trend was obtained in this study when the contact angle was measured with PF adhesive (i.e., $\theta_{\mathrm{i}}$ was higher on SPIHMR than on SPC). The PF adhesive drop, which was applied on the treated wood surfaces, behaved differently than a water drop. An initial contact angle of PF was always higher than that of water, except on a SPI surface, where $\theta_{\mathrm{i}}$ was practically the same (Table 5.8).

Table 5.8. Contact angle (degree) on treated wood surfaces as a function of time and treatment. Data is an average of 12 measurements.

\begin{tabular}{|c|c|c|c|c|c|c|c|c|}
\hline \multicolumn{9}{|c|}{ Liquid } \\
\hline \multirow[b]{2}{*}{ Treatment } & \multicolumn{7}{|c|}{ Water } & \multirow{2}{*}{$\begin{array}{c}\text { PF Adhesive } \\
\theta_{\mathrm{i}}(\mathbf{0} \mathrm{s} .)\end{array}$} \\
\hline & $\theta_{\mathrm{i}}(\mathbf{0} \mathrm{s})$. & $10 \mathrm{~s}$. & $20 \mathrm{~s}$. & $30 \mathrm{~s}$. & $40 \mathrm{~s}$. & $50 \mathrm{~s}$. & $60 \mathrm{~s}$. & \\
\hline SPC & 45.7 & 13.2 & 7.5 & 6.1 & 4.5 & 3.7 & 2.8 & 74.6 \\
\hline SPI & 100.8 & 99.5 & 98.4 & 98.2 & 97.6 & 97.0 & 97.0 & 100.6 \\
\hline SPIHMR & 30.0 & 3.5 & 1.2 & 0.0 & 0.0 & 0.0 & 0.0 & 93.5 \\
\hline SPIXY & 84.3 & 64.9 & 57.3 & 54.0 & 51.8 & 49.8 & 47.0 & 109.3 \\
\hline SPINA & 53.3 & 12.1 & 4.7 & 0.0 & 0.0 & 0.0 & 0.0 & 88.3 \\
\hline SPIXYNA & 72.4 & 25.4 & 15.4 & 9.6 & 6.9 & 5.3 & 3.6 & 90.6 \\
\hline
\end{tabular}

Since water has a higher surface free energy $\left(72.8 \mathrm{~mJ} / \mathrm{m}^{2}\right)$ than PF adhesives $\left(52 \mathrm{~mJ} / \mathrm{m}^{2}\right)$ (Gardner et al. 2000), $\theta_{\mathrm{i}}$ of a water drop should be higher than that of PF adhesives. This relationship is expected from Young's equation (Equation 2.2). A lower liquid surface tension forms a lower contact angle in the solid/liquid system (Shi and Gardner 2001). However, this was not confirmed by the results of this study: instead, the PF adhesive with a low surface tension formed a higher contact angle. The deviation can be attributed to high viscosity of the PF adhesive, which impeded the complete manifestation of the surface forces in a short time. 
Additionally, the contact angle of water on the wood surface was not in equilibrium-it changed over time-and so the expectation to obtain similar data to that originating from the thermodynamic principles is not relevant. Moreover, surface free energy comprises polar and dispersive components (Garnier and Glasser 1994). The polar component of surface free energy of water is $51 \mathrm{~mJ} / \mathrm{m}^{2}$, and the dispersive component is $21.8 \mathrm{~mJ} / \mathrm{m}^{2}$ (Gindl et al. 2001; Zhang et al. 1997). The proportion between a polar component and a dispersive component of surface free energy of PF adhesive differs from water. This might contribute to the unexpected deviation of $\theta_{i}$ for water and PF in regard to the wood surface treatment. Gardner et al. (2000) found that equilibrium contact angle of PF and PMDI adhesives decreased with increasing dispersive surface energy of wood samples.

\subsubsection{Critical Surface Tension}

The principle of critical surface tension $\left(\gamma_{c}\right)$ could be applied only to SPI and SPIXY samples. Other samples had higher surface tension, which caused the liquid probes bromonaphthalene and ethylene glycol, with surface tensions of 44.6 and $48.3 \mathrm{mN} / \mathrm{m}$ respectively, to spread spontaneously after the drop was applied. Thus, it was not possible to measure contact angles in these cases. To illustrate non-equilibrium conditions of a water drop applied on the wood surface, the relative change of $\theta$ between 0 and 60 seconds is shown in Figure 5.7 for each of the surface treatments. 


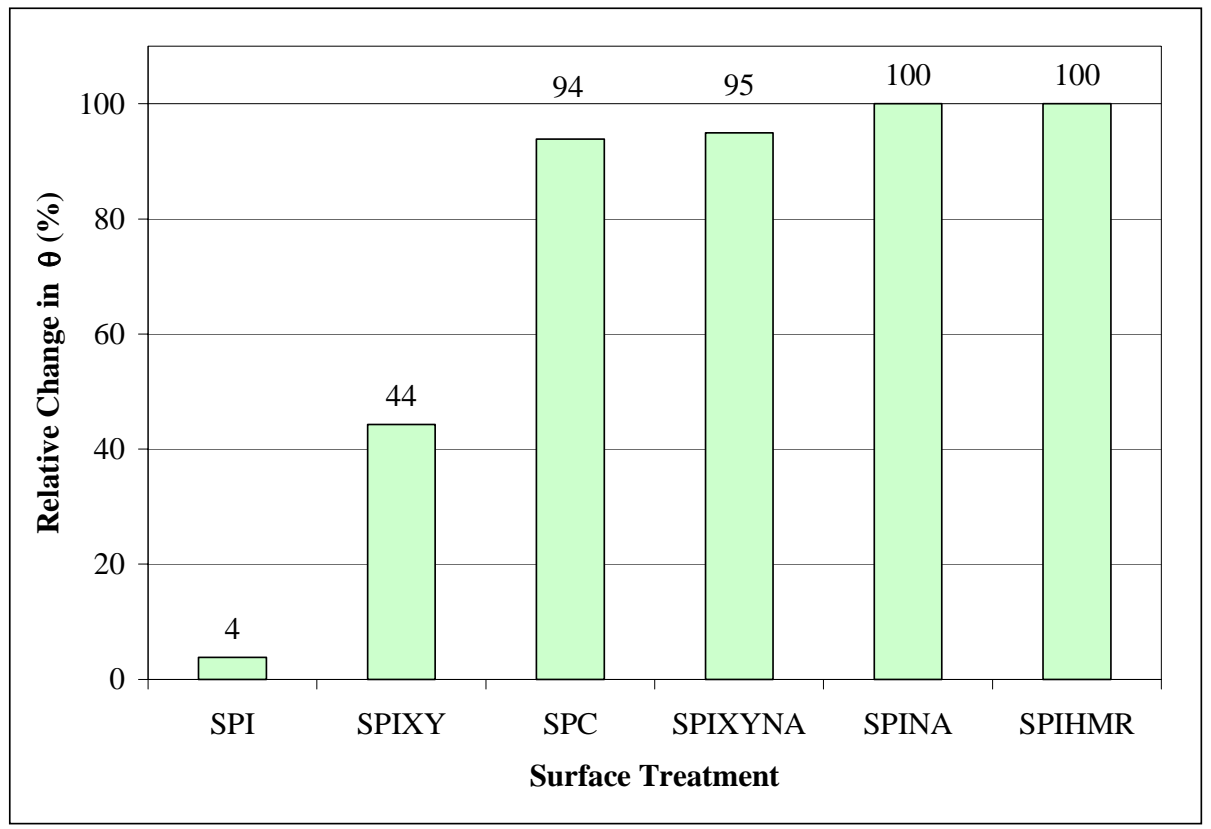

Figure 5.7. The relative change in the contact angle during one minute.

When water was applied on the inactivated surface, the contact angle stayed almost constant during observation-it changed by only 4\%. The change was moderate for the SPIXY sample (44\%). All other samples exhibited dramatic change in the water contact angle (e.g., 94$100 \%$ ) over one minute. When other probe liquids from Table 5.5 were applied, the contact angle changes even faster on these surfaces. Therefore, $\gamma_{c}$ was evaluated only for SPI and SPIXY samples, since their surfaces provided quite stable contact angles for all liquid probes used. However, the obtained values of $\theta$ were not true equilibrium values, but only an approximation.

A graphic presentation was used to evaluate $\gamma_{c}$. First, the cosine of the initial contact angles, measured for probe liquids on the SPI and SPIXY surfaces, was evaluated. The results are shown in Table 5.9. Then, the $\cos \theta_{\mathrm{i}}$ of the probe liquids was plotted against surface tension. The data were fit with a linear line, which was extrapolated to the point where $\cos \theta$ equals unity. The $\mathrm{x}$ coordinate of this point represents $\gamma_{c}$. The value of $\gamma_{c}$ was calculated from the empirical equation obtained from the regression line in Figure 5.8 and Figure 5.9. 
Table 5.9. Relationship between surface tensions of probe liquids and $\theta_{i}$.

\begin{tabular}{|c|c|c|c|c|c|}
\hline \multirow{3}{*}{$\begin{array}{l}\text { Liquid } \\
\text { Probe }\end{array}$} & \multirow{3}{*}{$\begin{array}{c}\text { Surface } \\
\text { Tension } \\
\text { mN/m }\end{array}$} & \multicolumn{4}{|c|}{ Sample } \\
\hline & & \multicolumn{2}{|c|}{ SPI } & \multicolumn{2}{|c|}{ SPIXY } \\
\hline & & $\theta_{\mathrm{i}}$ & $\operatorname{Cos} \theta_{i}$ & $\theta_{\mathrm{i}}$ & $\operatorname{Cos} \theta_{i}$ \\
\hline Water, deionized & 72.8 & 100.8 & -0.19 & 84.3 & 0.10 \\
\hline Glycerol & 64.0 & 83.0 & 0.12 & 78.2 & 0.20 \\
\hline Formamide & 58.3 & 55.1 & 0.57 & 57.3 & 0.54 \\
\hline Ethylene glycol & 48.3 & 46.4 & 0.69 & 48.3 & 0.67 \\
\hline Bromonaphthalene & 44.6 & 7.7 & 0.99 & 14.2 & 0.97 \\
\hline
\end{tabular}

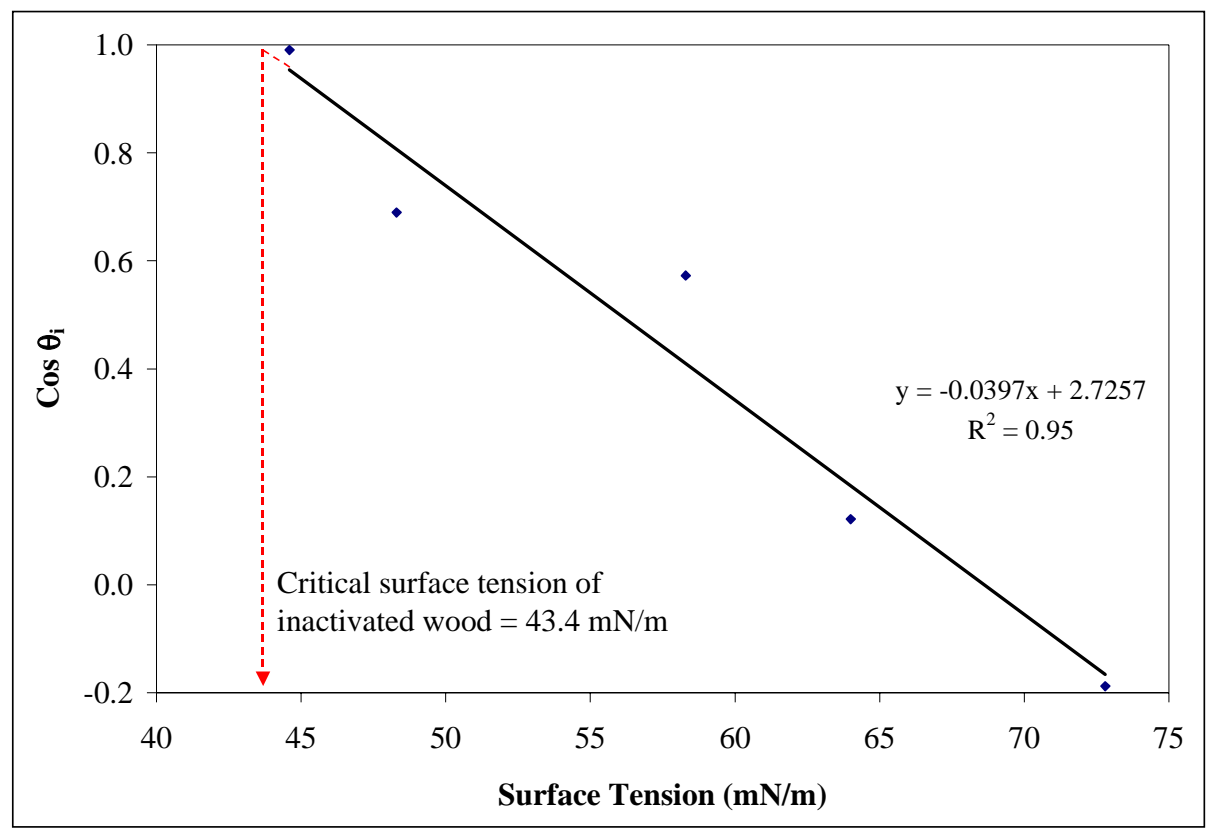

Figure 5.8. Critical surface tension plot for inactivated wood surface. 


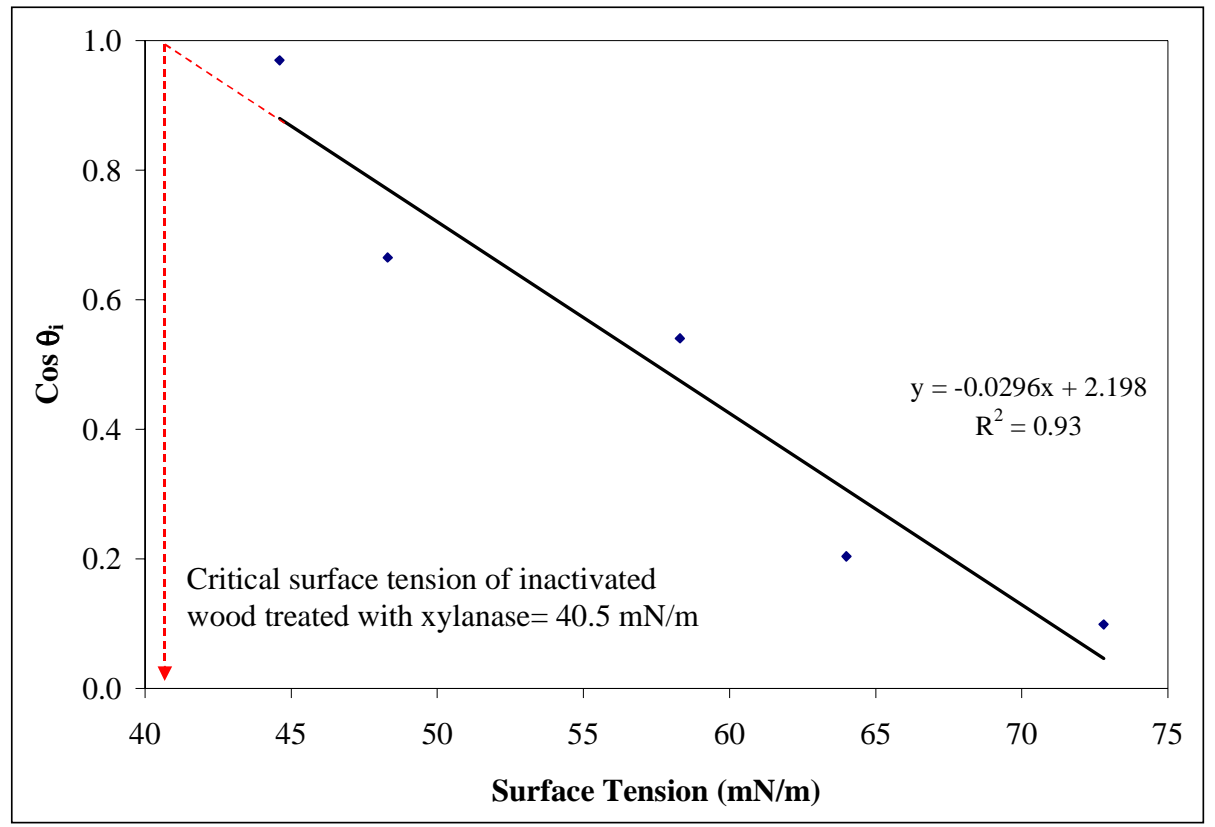

Figure 5.9. Critical surface tension plot for inactivated wood surface treated with xylanase.

The results showed that the SPI sample exhibited a slightly higher $\gamma_{c}$ (i.e., $43.4 \mathrm{mN} / \mathrm{m}$ ) than SPIXY (i.e., $40.5 \mathrm{mN} / \mathrm{m}$ ) but the difference was not significant. Both samples had substantially lower values of $\gamma_{c}$ than fresh wood surfaces, usually in the range of $50-60 \mathrm{mN} / \mathrm{m}$ (Gardner et al. 1991a; Mantanis and Young 1997). Nguyen and Johns (1978) estimated $\gamma_{c}$ of 52.9 $\mathrm{mN} / \mathrm{m}$ for Douglas-fir. Scheikl and Dunky (1998) found that pine (Pinus Silvestris L.), with 3\% $\mathrm{MC}$, had $\gamma_{\mathrm{c}}$ of $51.9 \mathrm{mN} / \mathrm{m}$ for earlywood and $52.3 \mathrm{mN} / \mathrm{m}$ for latewood.

One can see that inactivated and xylanase-treated surfaces exhibited approximately 20 to $30 \%$ lower $\gamma_{c}$ than fresh surfaces investigated in other studies. Consequently, adhesive wettability is low on such surfaces, which potentially means a poor adhesive bond. Indeed, a low $\gamma_{c}$ of SPI and SPIXY samples can be attributed (beside a low $\mathrm{pH}$ of these surfaces) to weak adhesion as shown in the following section. 


\subsubsection{Effect of Surface Treatment on Adhesion}

The results of fracture test measurements are shown in Appendix L and Appendix M. Detailed statistical analysis provided the results for significant differences in $G_{\max }$ among the surface treatments. The Duncan multiple range test with a $95 \%$ confidence level was used. The result, as generated by statistical software STATGRAF, was used to identify statistically significant differences.

\subsubsection{Specimens Bonded with PVA Adhesive}

The $\mathrm{G}_{\max }$ of PVA bonded specimens is shown in Table 5.10. A star (*) denotes a statistically significant difference. The affect of southern pine surface treatment on adhesion (SERR) of PVA adhesive is shown in Figure 5.10.

Table 5.10. Statistically significant differences in $G_{\text {max }}$ of PVA adhesive among surface treatments (denoted with *).

\begin{tabular}{|c|c|c|c|c|c|}
\hline \multirow{2}{*}{$\begin{array}{l}\text { Sample } \\
\text { Name }\end{array}$} & \multirow{2}{*}{$\begin{array}{l}\text { Number of } \\
\text { Observation }\end{array}$} & \multirow[t]{2}{*}{ Gmax } & \multicolumn{3}{|c|}{ Homogeneous Groups } \\
\hline & & & A & B $\quad$ C & \\
\hline SPI & 8 & 50.4 & $\mathrm{X}$ & & \\
\hline SPIXY & 8 & 66.7 & $\mathrm{X}$ & & \\
\hline SP IXYNA & 8 & 114.9 & & $\mathrm{X}$ & \\
\hline SPINA & 8 & 123.8 & & $\mathrm{X}$ & \\
\hline $\mathrm{SPC}$ & 8 & 260.3 & & $\mathrm{X}$ & \\
\hline Contrast & & & & Differen & nce \\
\hline SPI & - SPIXY & & & $-16 \cdot 3$ & \\
\hline SPI & - SPIXYNA & & & -64.5 & $\star$ \\
\hline SPI & - SPINA & & & -73.3 & $\star$ \\
\hline SPI & $-\mathrm{SPC}$ & & & -209.8 & $\star$ \\
\hline SPIXY & - SPIXYNA & & & -48.2 & \\
\hline SPIXY & - SPINA & & & -57.0 & * \\
\hline SPIXY & $-\mathrm{SPC}$ & & & -193.5 & $\star$ \\
\hline SPIXYNA & - SPINA & & & -8.9 & \\
\hline SPIXYNA & $-\mathrm{SPC}$ & & & -145.4 & $\star$ \\
\hline SP INA & $-\mathrm{SPC}$ & & & -136.5 & $\star$ \\
\hline
\end{tabular}




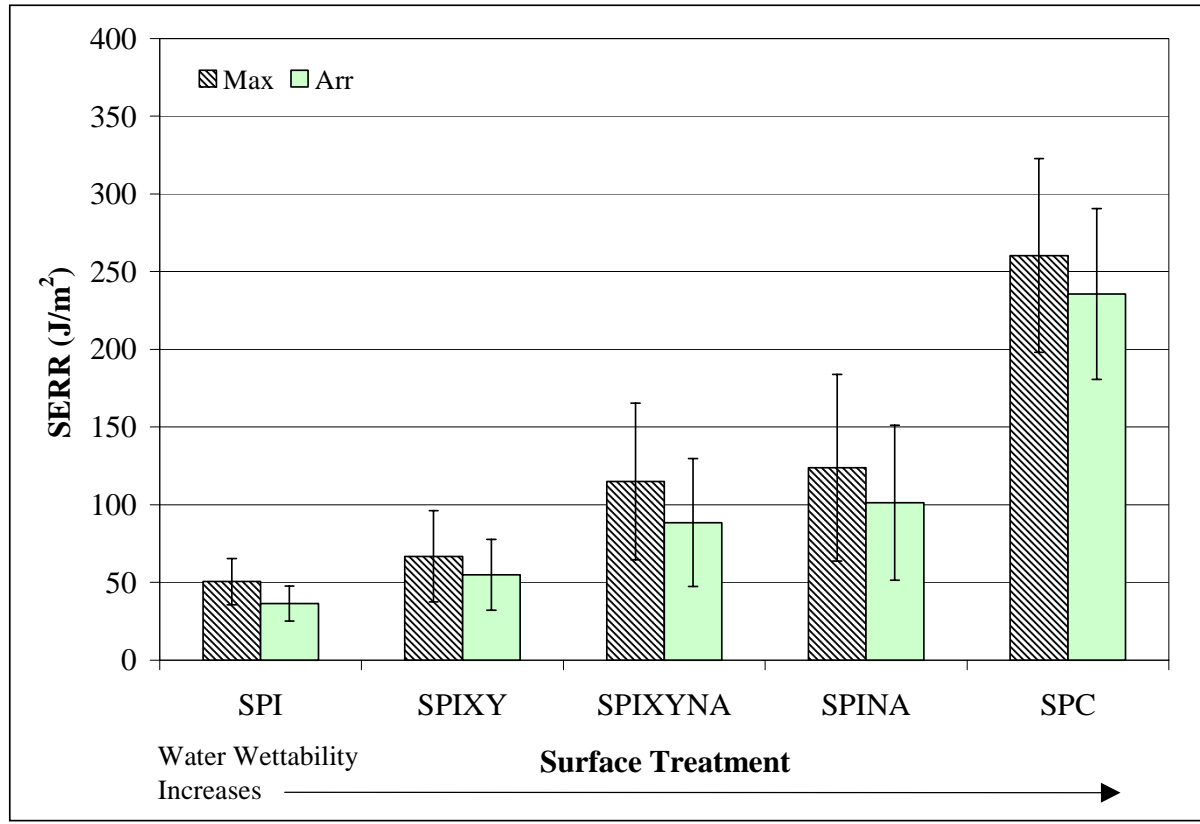

Figure 5.10. Effect of southern pine surface treatment on SERR of PVA adhesive.

Surface treatments by aqueous solution of different chemicals affected PVA adhesion in several different ways. The inactivated sample exhibited the lowest adhesion, as indicated by $\mathrm{G}_{\max }$. The treatment of SPI sample with xylanase (SPIXY) increased PVA adhesion, but not significantly. The adhesion increased significantly, when these surfaces were washed with $\mathrm{NaOH}$. These specimens (SPIXYNA) exhibited more than a double increase in the $\mathrm{G}_{\max }$. The $\mathrm{G}_{\max }$ was even greater with a surface treatment of $\mathrm{NaOH}$ alone.

Alkali treatment with $\mathrm{NaOH}$ consistently improved surface wettability. Enzymatic treatment with xylanase was probably unsuccessful because the surface was covered with components (i.e., extractives and degraded VOCs) they cannot hydrolyze. These enzymes are able to degrade xylan polymers in wood (Schönberg et al. 2001). Xylanase are aimed to treat inactivation caused by the hornification mechanism. Therefore, one cannot expect a significant reactivation effect of xylanase when the surface inactivation originates from extractives or VOCs. 
An insignificant effect of xylanases on adhesion could also be attributed to a low activity of xylanases at $\mathrm{pH}$ of around 5. The highest activity of xylanases is expected when the $\mathrm{pH}$ value is between 7.5 and 9.2 (Gupta et al. 2000). The inactivated wood surface, which was acidic, hindered the xylanases activity. Another factor that lowered xylanase activity was water evaporation. Since water evaporated very fast from the wood surface, xylanases remained there without mobility.

PVA adhesive bond failed always cohesively. In spite of some improvements in adhesion of the PVA bonded samples due to surface treatments, the highest achieved $G_{\max }$ within the treated samples (i.e., SPINA) presented less than half of the $G_{\max }$ obtained for the control samples. Therefore, surface treatments of PVA bonded samples were unsuccessful. However, an interesting relationship was again found between adhesion and wettability. $G_{\max }$ of the PVA bonded sample increased with water wettability. The relationship was not linear but rather polynomial (second order), which explained most of the variability in data (99\%). The relationship between $\mathrm{G}_{\max }$ and $\cos \theta_{\mathrm{i}}$ is shown in Figure 5.11 .

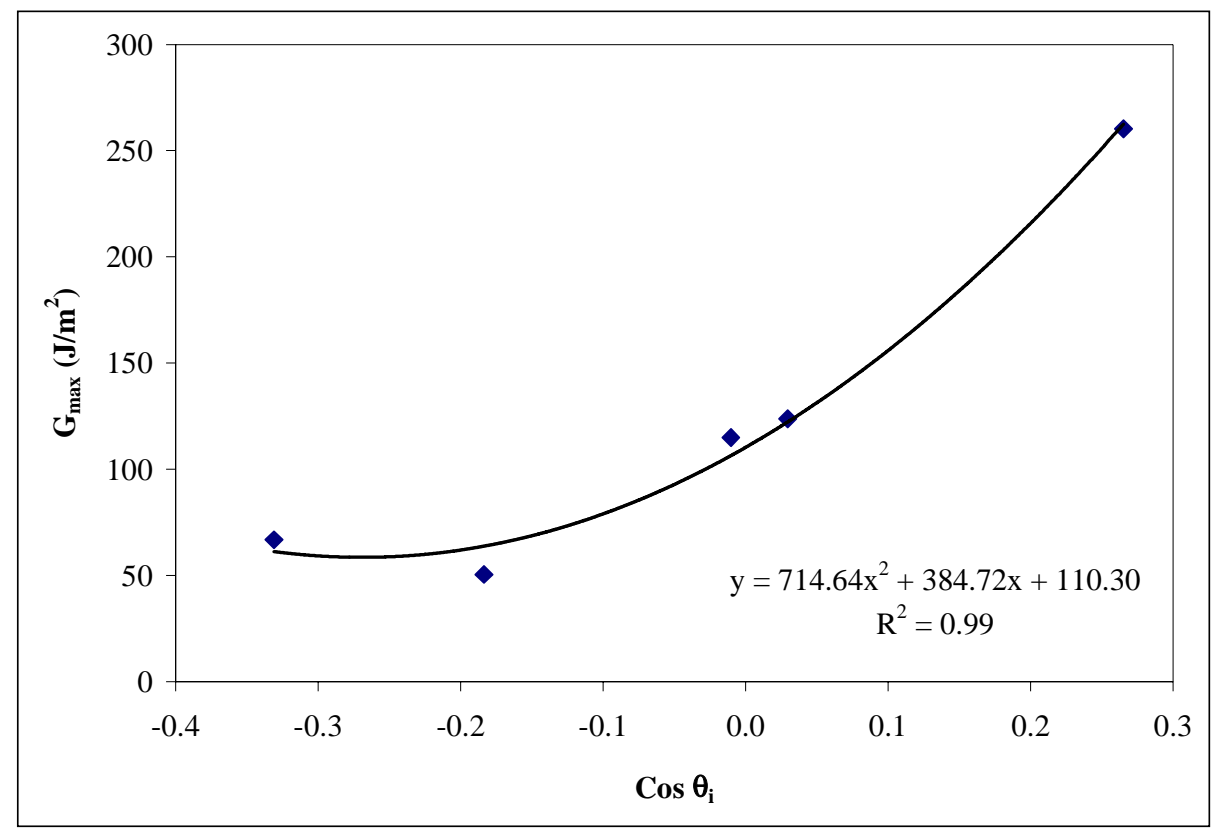

Figure 5.11. Relationship between PVA adhesion and water wettability. 


\subsubsection{Specimens Bonded with PF Adhesive}

The result of statistical analysis, which indicated significant differences in $G_{\max }$ of $P F$ adhesive, is shown in Table 5.11.

Table 5.11. Statistically significant differences in $G_{\text {max }}$ of PF adhesive among surface treatments (denoted with *).

\begin{tabular}{|c|c|c|c|c|c|}
\hline \multirow{2}{*}{$\begin{array}{l}\text { Sample } \\
\text { Name }\end{array}$} & \multirow{2}{*}{$\begin{array}{l}\text { Number of } \\
\text { Observation }\end{array}$} & \multirow[t]{2}{*}{ Gmax } & \multicolumn{3}{|c|}{ Homogeneous Groups ${ }^{a}$} \\
\hline & & & A & B $\mathrm{C}$ & \\
\hline SPIXY & 8 & 52.4 & $\mathrm{x}$ & & \\
\hline SP I & 8 & 61.1 & $\mathrm{X}$ & & \\
\hline SP IHMR & 8 & 83.5 & $\mathrm{X}$ & & \\
\hline SP IXYNA & 8 & 188.6 & & $\mathrm{X}$ & \\
\hline SP INA & 8 & 190.7 & & $\mathrm{X}$ & \\
\hline $\mathrm{SPC}$ & 8 & 248.3 & & $\mathrm{X}$ & \\
\hline \multicolumn{3}{|l|}{ Contrast } & \multicolumn{3}{|c|}{ Difference } \\
\hline SPI & - SPIXY & & & 8.6 & \\
\hline SPI & - SPIXYNA & & & -127.5 & * \\
\hline SPI & - SPINA & & & $-129 \cdot 6$ & $\star$ \\
\hline SPI & $-\mathrm{SPC}$ & & & -187.3 & * \\
\hline SPI & - SPIHMR & & & -22.4 & \\
\hline SPIXY & - SPIXYNA & & & -136.1 & * \\
\hline SPIXY & - SPINA & & & -138.2 & * \\
\hline SPIXY & $-\mathrm{SPC}$ & & & -195.9 & * \\
\hline SPIXY & - SPIHMR & & & -31.0 & \\
\hline SP IXYNA & - SPINA & & & -2.1 & \\
\hline SPIXYNA & $-\mathrm{SPC}$ & & & -59.7 & $\star$ \\
\hline SP IXYNA & - SPIHMR & & & 105.1 & $\star$ \\
\hline SPINA & $-\mathrm{SPC}$ & & & -57.6 & $\star$ \\
\hline SPINA & - SPIHMR & & & 107.2 & $\star$ \\
\hline $\mathrm{SPC}$ & - SPIHMR & & & 164.9 & $\star$ \\
\hline
\end{tabular}

The effect of surface treatment on adhesion of specimens bonded with PF adhesive differed from PVA bonded specimens. Significant improvement of $G_{\max }$ was obtained only for surface treatments that included $\mathrm{NaOH}$ (SPIXYNA and SPINA). However, these values were still significantly lower than $\mathrm{G}_{\max }$ exhibited by the control specimens. HMR and enzymatic treatments were unsuccessful in restoring the bondability of inactivated surfaces with PF adhesive. SPIHMR and SPIXY specimens exhibited a low $\mathrm{G}_{\max }, 83$ and $52 \mathrm{~J} / \mathrm{m}^{2}$, respectively. 
However, when SPXY was additionally treated with $\mathrm{NaOH}$, the $\mathrm{G}_{\max }$ of these specimens increased to the value of $189 \mathrm{~J} / \mathrm{m}^{2}$. Even a higher $\mathrm{G}_{\max }$ of $191 \mathrm{~J} / \mathrm{m}^{2}$ was achieved when $\mathrm{NaOH}$ was used solely on the inactivated surface. This treatment reached $76 \%$ of the maximum possible adhesion (SERR) achieved with the fresh surface of the control (SPC) sample (Figure 5.12).

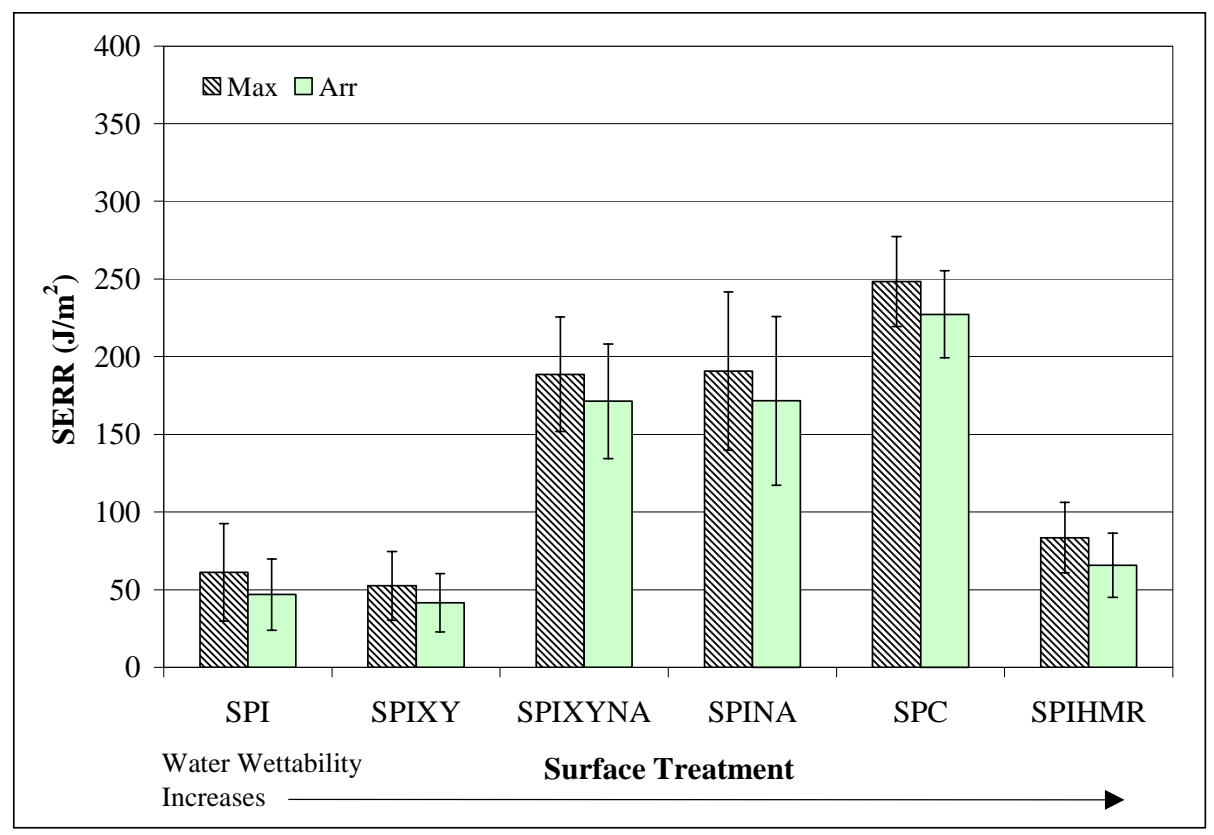

Figure 5.12. Effect of southern pine surface treatment on SERR of PF adhesive.

A significant effect of $\mathrm{NaOH}$ treatment on PF adhesion can be mainly (besides improved wettability due to $\mathrm{NaOH}$ ) attributed to its neutralization effect of the inactivated SP surfaces. The $\mathrm{pH}$ value of SP extractives, which were washed from these surfaces with water with acetone:water (9:1) ranged from 3.5 to 4 . The application of $\mathrm{NaOH}$ increased $\mathrm{pH}$ and neutralized surfaces. Neutralized extractives/VOCs have improved solubility in water and can be expected to be removed as salts during washing. This can be expected to result in enhanced adhesion. The surface treatment with xylanase was not effective in restoring $G_{\max }$ of $P F$ for the same reasons that applied in the case of PVA adhesive. Additionally, the SPIXY surface remained acidic after treatment, since the solution of enzymes had $\mathrm{pH}$ of 5. An acid condition retarded or even restrained adequate curing of $\mathrm{PF}$ adhesive that requires alkali condition for polymerization. The effect of undercured PF adhesive of SPIXY specimens is reflected in the lowest $\mathrm{G}_{\max }$. 
The HMR treatment was also not very operative. However, HMR was developed as a coupling agent to improve bondline resistance to moisture, not for dry strength (Vick et al. 1998). It is assumed that activity of HMR was limited when in contact with the acidic SP surface, which caused the drop in $\mathrm{pH}$ value. HMR coupling agent is active under alkaline conditions, but not in acidic. However, HMR treated surfaces bonded with PF adhesive had $G_{\max }$ of $83 \mathrm{~J} / \mathrm{m}^{2}$, which is $22 \mathrm{~J} / \mathrm{m}^{2}$ higher than obtained with inactivated samples. This improvement might be due to the better wettability and neutralizing effect of HMR, which contained $2.44 \%$ of $\mathrm{NaOH}$. Therefore, surfaces of SPIHMR were not as acidic as SPI and the cure of PF adhesive was less retarded.

A surface treatment with $\mathrm{NaOH}$ was an effective remedy for inactivated wood surfaces when bonded with PF adhesive, since the adhesive bond performance increased by a factor of three compared with the untreated inactivated surface. A significant increase in $\mathrm{G}_{\max }$ of surfaces treated with $\mathrm{NaOH}$ and bonded with PF (SPIXYNA and SPINA) is attributed to better wettability and neutralizing effect.

$\mathrm{NaOH}$ improved surface wettability, which often leads to better adhesion (Hancock 1963; USDA 1999; Wellons 1977). Also, washing with $\mathrm{NaOH}$ removed some of the extractives concentrated on the surface. One should note that the color of the wood surface changed also after treatment, which can be attributed either to removal of some substances or neutralizing of the surface. The later case was obviously present there, since $\mathrm{NaOH}$ is an alkaloid with a $\mathrm{pH}$ of 14, which neutralized the acid character of inactivated wood surface. Surface treatment with $\mathrm{NaOH}$ increased the $\mathrm{pH}$ value of several acidic tropical woods (Chen 1970). Neutral or alkaline conditions were beneficial for the cure of the PF adhesive, since this study used alkali catalyzed PF adhesive. Thus, the cure process proceeded more adequately due to the neutralized surface acidity, which often retards or prolongs a curing of this type of PF adhesive (Hse and Kuo 1988). Otherwise, PF adhesives can polymerize either under acid or alkaline conditions (Pizzi 1983). The reaction of PF adhesive on SPIXYNA and SPINA specimens was probably accelerated due to higher $\mathrm{pH}$ originated from $\mathrm{NaOH}$. Gardner and Elder (1988) found that gel time of $\mathrm{PF}$ adhesive was reduced when $\mathrm{NaOH}$ was applied on southern pine particles. 
The difference in $\mathrm{G}_{\max }$ of SPINA between PVA and PF bonded samples support the fact, that $\mathrm{pH}$ had an impact on $\mathrm{G}_{\max }$ of inactivate specimens bonded with $\mathrm{PF}$ adhesive. One can see that PVA adhesive provided approximately $2 / 3$ of $G_{\max }$ that PF adhesive did for SPINA. But earlier, both adhesives performed similarly on control surfaces. Precisely, $G_{\max }$ of PVA and PF on SPC surfaces was 260.3 and $248.3 \mathrm{~J} / \mathrm{m}^{2}$, respectively. One can summarize that only those adhesives that are $\mathrm{pH}$-sensitive responded to re-activation.

The correlation between the water wettability of the treated surfaces and $G_{\max }$ of PF adhesive was poor. Actually, $\mathrm{G}_{\max }$ tended to increase with increased wettability to the SPC sample, but then decreased drastically for the SPIHMR sample. However, when wettability was evaluated with PF adhesive, a strong linear relationship was observed between $G_{\max }$ of PF adhesive bond and $\cos \theta_{\mathrm{i}}$ (Figure 5.13).

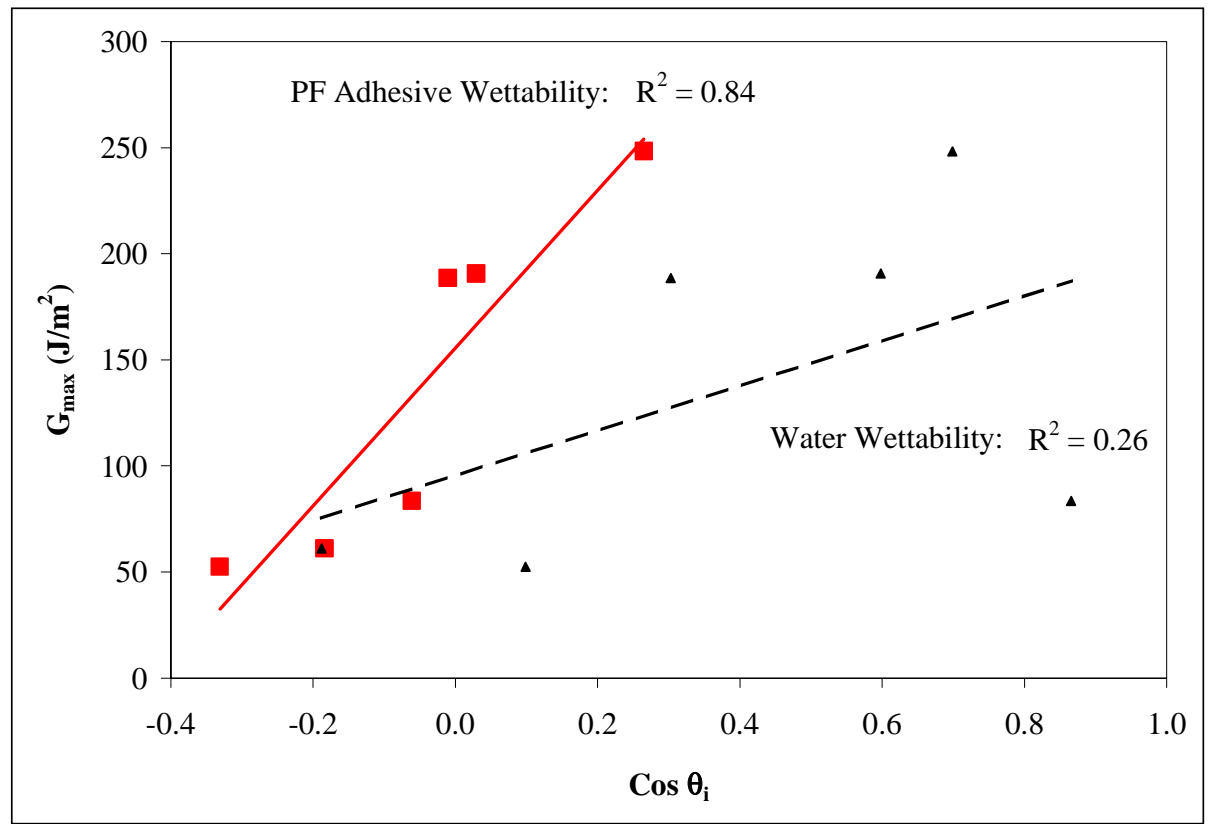

Figure 5.13. Relationship between PF adhesion and wood surface wettability.

Since the severity of wood surface inactivation is often evaluated by measurement of water wettability, the presented relationship suggests that the inactivation should rather be evaluated by wettability measurement using the adhesive. The $\theta_{i}$ of PF adhesive might mostly be related to the initial change in $\mathrm{pH}$. 


\subsubsection{Effect of Adhesive on $\mathrm{G}_{\max }$ of Inactivated Specimens}

The established adhesion between two adherends and an adhesive is not only a function of the adherends surface. The choice of the adhesive drastically impacted the adhesion of bonded assemblies. A statistically significant difference in $\mathrm{G}_{\max }$ among adhesives is shown in Table 5.12.

Table 5.12. Statistically significant differences in $G_{\text {max }}$ of inactivated SP surface among adhesives (denoted with *).

\begin{tabular}{|c|c|c|c|c|}
\hline \multirow{2}{*}{$\begin{array}{l}\text { Sample } \\
\text { Name }\end{array}$} & \multirow{2}{*}{$\begin{array}{l}\text { Number of } \\
\text { Observation }\end{array}$} & \multirow{2}{*}{ Gmax } & \multicolumn{2}{|c|}{ Homogeneous Groups ${ }^{a}$} \\
\hline & & & $A$ & B $\mathrm{C}$ \\
\hline PFHMR & 8 & 27.9 & $\mathrm{x}$ & \\
\hline PVA & 8 & 50.4 & $\mathrm{x}$ & $\mathrm{X}$ \\
\hline $\mathrm{PF}$ & 8 & 61.1 & & $\mathrm{X}$ \\
\hline PMDI & 8 & 169.3 & & $\mathrm{X}$ \\
\hline \multicolumn{3}{|c|}{ Contrast } & \multicolumn{2}{|r|}{ Difference } \\
\hline PVA & \multicolumn{2}{|l|}{$-\mathrm{PF}$} & \multicolumn{2}{|r|}{-10.6} \\
\hline PVA & \multicolumn{2}{|l|}{ - PFHMR } & \multicolumn{2}{|r|}{22.6} \\
\hline PVA & \multicolumn{2}{|l|}{ - PMDI } & \multicolumn{2}{|r|}{$-118.9 \star$} \\
\hline $\mathrm{PF}$ & \multicolumn{2}{|l|}{ - PFHMR } & \multicolumn{2}{|r|}{$33.2 *$} \\
\hline $\mathrm{PF}$ & \multicolumn{2}{|l|}{- PMDI } & \multicolumn{2}{|r|}{$-108.2 \star$} \\
\hline PFHMR & \multicolumn{2}{|l|}{ - PMDI } & \multicolumn{2}{|r|}{$-141.4 \star$} \\
\hline
\end{tabular}

Adhesive properties, such as molecular weight, viscosity, $\mathrm{pH}$, and solids content influences adhesion after adhesive cure (Marra 1992). The statistically significant differences in $\mathrm{G}_{\max }$ were indicated between the specimens bonded with PFHMR adhesive and the specimens bonded with PF or PMDI adhesives. PVA bonded specimens provided moderate $\mathrm{G}_{\max }$, which differed only from the PMDI bonded specimens. The specimens bonded with PFHMR adhesive exhibited the lowest adhesive bond performance. $G_{\max }$ of these specimens was practically negligible. The adhesive bond failed without any wood failure. The surface of the broken adhesive bond was smooth with the imprint of the opposite adherend. $G_{\max }$ of PVA and PF adhesives was higher than that of PFHMR, but still substantially lower than the PMDI value. A significant increase in $\mathrm{G}_{\max }$ was observed for the inactivated SP specimens bonded with PMDI adhesive. The effect of adhesive mixture on SERR of adhesive bond is shown in Figure 5.14. 


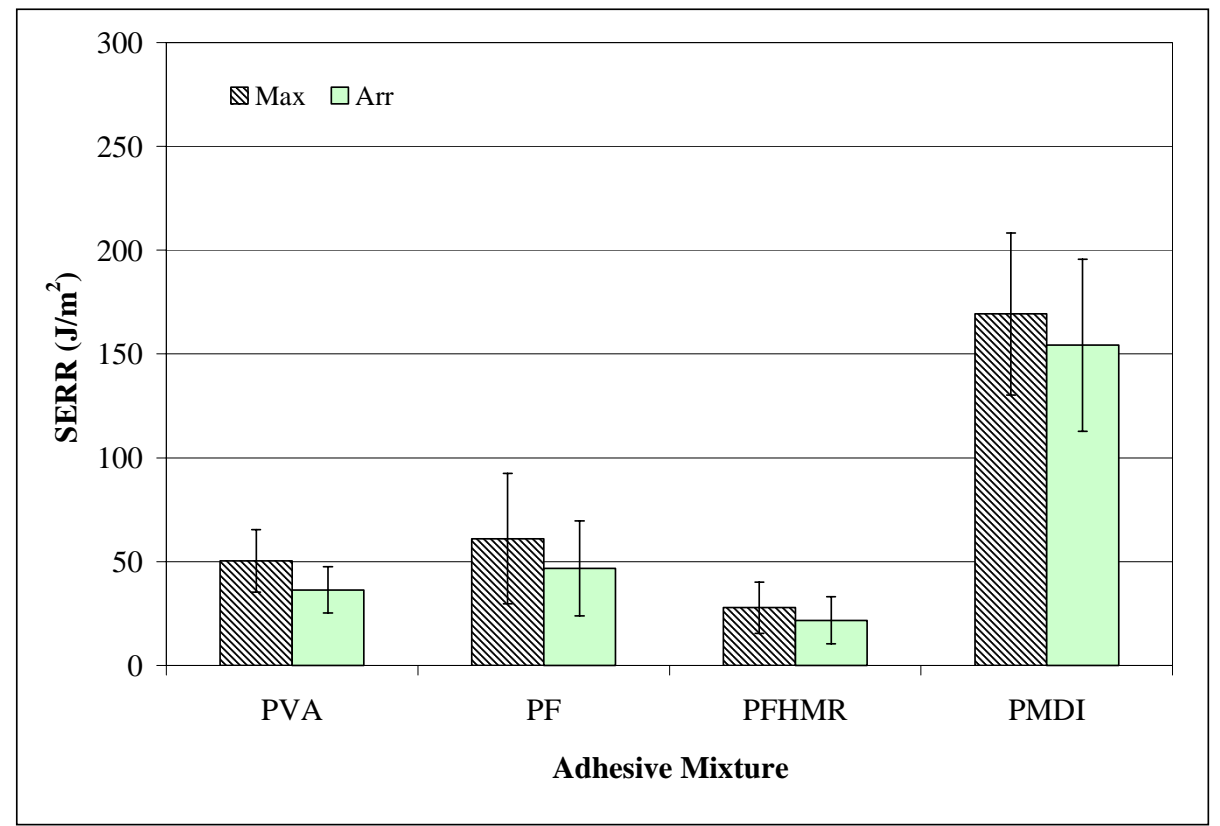

Figure 5.14. Effect of adhesive mixture on SERR of bonded SPI specimens.

A low bonding performance of PF and PVA adhesive when bonded with inactivated wood surfaces was expected. This was in agreement with the results of previous studies (Christiansen 1990; USDA 1999). A drastically low $\mathrm{G}_{\max }$ of PFHMR bonded specimens was a surprise. However, when compared with the PF bonded sample, one can see that PFHMR adhesive had substantially lower solids content than PF adhesive. The role of HMR included in PF adhesive remains unclear. It seems that HMR did not provide any benefits to the PFHMR adhesive mixture. Just the opposite, its inclusion reduced solids content up to the limit, which significantly lowered the viscosity and consistency of the adhesive itself.

A surprisingly high $\mathrm{G}_{\max }$ was observed for the inactivated specimens bonded with PMDI adhesive. Zheng and Frazier (2002) found that PMDI provided much lower adhesion than PF adhesive when bonded with yellow-poplar. This behavior was attributed to the adhesive bond thickness-PMDI had the thinnest bondline. PMDI usually has a low average molecular weight and a low viscosity. Thus, it can penetrate wood surfaces easily, which sometimes results in a starved bondline. In this case, the adhesive penetrates too deep and to the places that do not contribute to adhesion. This ability for deep penetration might be beneficial when bonding an inactivated wood surface. PMDI adhesive probably could penetrate the inactivated surface layer 
and establish some connections with wood polymers. Since inactivation partially restrains adhesive penetration, excessive PMDI penetration did not occur. The bondline was thicker than in the case of bonding with a fresh wood surface.

A bondline thickness can impact $G_{\max }$ (Ebewele et al. 1979). Blackman et al. (1991) reported that strain energy release rate initially increases with the bondline thickness until it reaches a maximum. After that point, an increase in the bondline thickness has a detrimental affect on adhesive bond performance (Zheng and Frazier 2002). Thus, variation in adhesive bond thickness in regard to adhesive type might affect the measurements of $G_{\max }$. Other factors, such as surface roughness, the nature of surface roughness, and wood anisotropy, can influence fracture energy of adhesive bonds (Ebewele et al. 1980). 


\subsection{Conclusions}

The chemical treatments improved the wettability of inactivated wood surfaces. Wettability of the treated surfaces does not necessarily correlate with adhesion, especially when evaluated with water. This suggests that the wettability should rather be evaluated by a contact angle measurement using the adhesive. The critical surface tension of inactivated wood surface was lower than that of a fresh wood surface reported in the literature.

A sufficient improvement in adhesion due to surface chemical treatment was not evident for specimens bonded with PVA. Enzymatic treatment with xylanases did not improve adhesion. The HMR coupling agent was not operative on inactivated surfaces bonded with PF adhesive. Sodium hydroxide $(\mathrm{NaOH})$ was the most effective in restoring bonding ability of $\mathrm{PF}$ adhesive with inactivated wood surfaces. The maximum strain energy release rate $\left(G_{\max }\right)$ of specimens treated with $\mathrm{NaOH}$ increased by a factor of three when compared with inactivated specimens. Of the chemical treatments employed by this study, $\mathrm{NaOH}$ was the most effective for improving adhesive bond performance, while HMR had the greatest influence on improving water wettability. This can be attributed to high alkalinity of HMR.

The choice of the adhesive drastically impacted the adhesion of bonded assemblies. The inclusion of HMR coupling agent into the PF adhesive mixture was unsuccessful in restoring the adhesion of inactivated wood surfaces. On the contrary, PMDI adhesive provided a three times higher $G_{\max }$ than PF adhesive. Since the influence of PMDI was similar to the affect of surface treatment with $\mathrm{NaOH}$, the remedy for wood surface inactivation should be based on the usage of the adhesive with a better performance.

A general conclusion is that reactivation of inactivated southern pine revolves around the acidic, non-polar, hydrophobic components on the surface, and is predictable based on measurements of surface energetics. 


\section{Chapter 6. Method for Detection of Wood Surface Inactivation}

\subsection{Introduction}

Wood surface inactivation can be detected and evaluated from several different aspects. Mechanical testing of adhesion between wood surfaces is the most relevant indication of thermally induced inactivation. A relative comparison of adhesion between freshly machined wood surfaces and heat-exposed wood surfaces provides sufficient evidence for identification of the severity of surface inactivation. Freshly produced surfaces exhibit the strongest adhesion, while the severely inactivated surfaces exhibit a weak adhesion. Therefore, observing the adhesion in situ either from a strength or an energy approach could serve as the most reliable method for detecting wood surface inactivation.

However, in situ measurement of the adhesive bond performance has at least two shortcomings. First, most of the mechanical tests are too complex. These tests usually require expensive equipment, knowledge about the stress distribution, and often a special specimen preparation. Second, a mechanical test can be employed only after the manufacturing process, thus an occurrence of the inactivated surface might be detected too late.

Several methods were proposed for detecting wood surface inactivation prior to bonding a wood with an adhesive (Chow 1971; Freeman 1959; Kadlec 1980; Troughton 2001; Walters 1973). However, a sufficient and simple method for determining wood surface inactivation is not available. Most of the proposed techniques use water to test wettability or absorbtivity of a wood surface. These methods determine how fast a water drop will wet, spread, and penetrate the wood. The results mostly address the expectations, which arise from the definition of wood surface inactivation. One should note that inactivation refers to the reduced ability of an adhesive to properly wet, spread, penetrate, and cure (USDA 1999). Sometimes the results of these methods are not directly applicable for different reasons. For example, the APA-The Engineered Wood Association developed a surface inactivation rating system, which is based on the comparison of absorption time for a drop of water on sanded and unsanded veneer surfaces. Their evaluation does not assure that absorption of adhesive will behave the same as water. 
Besides this, several other deficiencies accompany these techniques. Walters (1973) used the APA surface inactivation index and wettability ratio to describe surface inactivation of veneer. The calculations are based on measurements of the time needed for a water drop to be absorbed on an unsanded (e.g., inactivated) veneer surface and a lightly sanded veneer surface. The measurements on the sanded surface served as a reference point. Since a knife cut a pristine veneer surface during peeling, unsanded and sanded surfaces differed in roughness. Roughness has significant impact on wood wettability, and it influences water adsorption into wood (USDA 1999). Thus, a measurement of water absorption on wood surfaces with different topography might not be adequate.

A similar impact of surface roughness on wettability arises when comparing the contact angle of the inactivated surface and the reference surface, which was modified by some other manner such as sanding, brushing, extraction, or chemical treatment (Chen 1970; Kadlec 1980). Besides this, many methods comprise either water absorption or water wettability for detection of inactivation and for prediction of adhesion potential. A simultaneous measurement of absorption and wettability would, therefore, provide more useful information on wettability and spreadability of a liquid drop on a wood surface.

Some other techniques proposed for quantifying wood surface inactivation are color intensity measurement or chemical analysis by spectroscopy (Chow 1971). The change in surface color is attributed to inactivation. Therefore, a relative comparison in color of the unscraped and scraped surface (Troughton 2001) provides useful information for the identification of wood surface inactivation. However, variations in wood color are present on fresh wood surfaces, and also, the change in a wood color might not always be directly related to surface inactivation, which makes this technique less reliable.

\subsubsection{Objectives}

The objective of the work described in this chapter was to establish a fast and reliable method for wood surface inactivation prior to bonding. 


\subsection{Material}

Heartwood samples of southern pine (SP) with MC around FSP were cut into tangential lamellas $(60 \times 320 \mathrm{~mm})$ and planed to the thickness of 12 or $10 \mathrm{~mm}$. Only wood without gross defects was chosen for sample preparation. Wood samples were sorted into the control group (SPC) and the inactivated group (SPI). Both groups were dried together in a convection oven at $200^{\circ} \mathrm{C}$ for 90 minutes to achieve $2 \% \mathrm{MC}$. The SPC sample comprised 4 lamellas, which were 12 $\mathrm{mm}$ thick and the SPI sample comprised 6 lamellas, which were $10 \mathrm{~mm}$ thick. Wood drying was carried out under the following drying parameters (Table 6.1).

Table 6.1. Properties of wood samples and drying parameters.

\begin{tabular}{lccc}
\hline $\begin{array}{c}\text { Wood Specification and } \\
\text { Drying Parameters }\end{array}$ & \multicolumn{2}{c}{ Sample } \\
\cline { 1 - 1 } Initial Average MC $(\%)$ & & SPC & SPI \\
Final Average MC $(\%)$ & & 25.5 & 25.2 \\
Max. Surface Temp. $\left({ }^{\circ} \mathrm{C}\right)$ & 196 & $<2$ \\
Drying Time (hrs:min) & $1: 30$ & 196 \\
Surface Modification & Removed by planing & None \\
Number of Lamellas & 4 & 6 \\
\hline
\end{tabular}

\subsubsection{Drying of Wood Samples}

The actual MC was controlled by the weight measurement of the samples during drying. A computer monitored the temperature of the wood surface of one SPI lamella every minute, while the temperature of the drying air was recorded manually every 10 minutes. The emission of volatile organic compounds (VOCs) was recognized when the surface temperature reached $130^{\circ} \mathrm{C}$. The intensity of VOCs emission increased with temperature. An occurrence of excessive VOCs emission, which appeared as smoke, was recognized when the surface temperature was above $150^{\circ} \mathrm{C}$. The wood surface reached this temperature in 40 minutes, when the average MC of the lamellas was below $8 \%$ (Figure 6.1). 


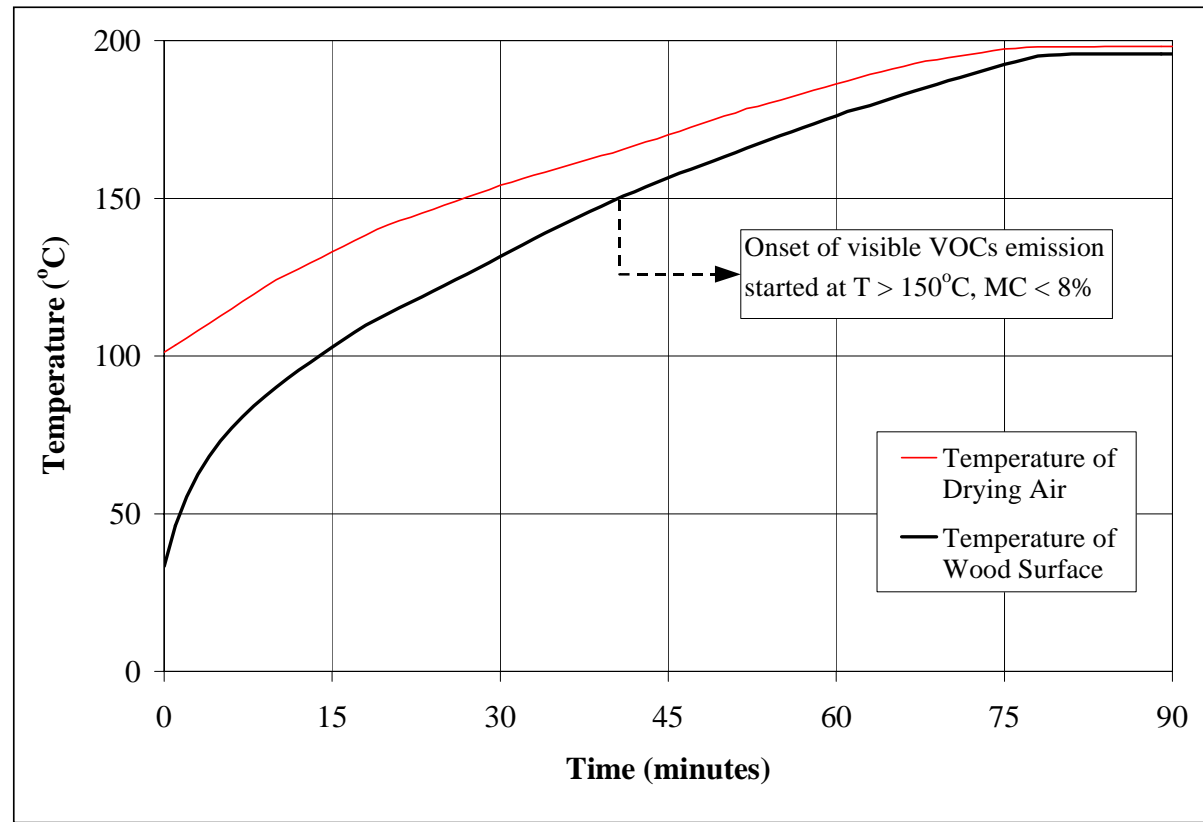

Figure 6.1. Changes in temperatures during southern pine lamellas drying.

After drying, the samples were cooled to room temperature. Dried SPI lamellas were stored in a plastic bag prior to contact angle and absorption measurements. Just before the measurements, a small area of 20x60 mm was removed by a planer on one side of each lamella (Figure 6.2). The measurements were then performed on the removed (i.e., reference) surface and on the not-removed (i.e., inactive) surface. After measurements, SPI lamellas were bonded for fracture testing. SPC lamellas, which were stored separately, were used only for fracture testing. The surface of these lamellas was removed by planing prior to bonding the fracture specimens. The contact angle and absorption were not measured for the SPC sample, since this was done on the removed (reference) surfaces of the SPI sample.

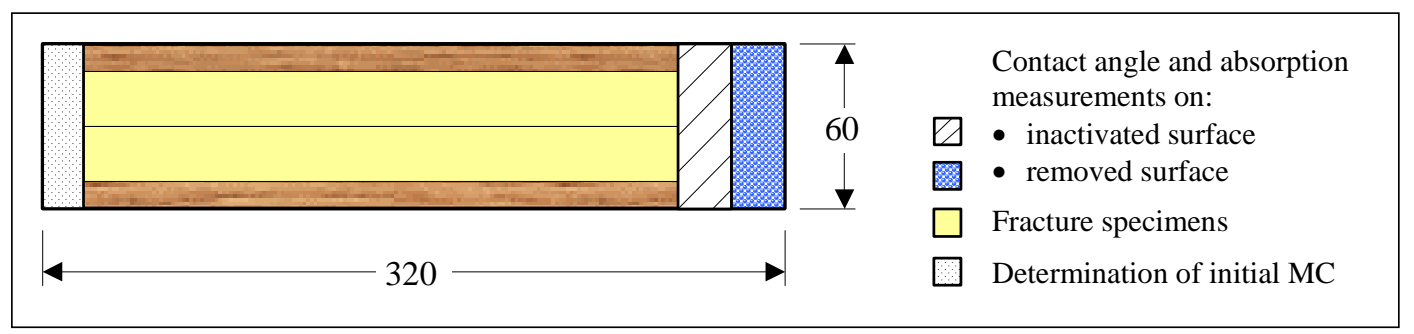

Figure 6.2. Specimen cutting diagram for each lamella. Width $(\mathrm{mm})$ is tangential direction. 


\subsection{Methods}

\subsubsection{Surface Inactivation Method}

This method evaluates wood surface inactivation prior to bonding. The method is based on a comparison of changes in a certain property (p), which is obtained from the studied surface (s) and on the control surface (c). The control surface should represent a surface that exhibits the extreme value (minimum or maximum) of a measured property. The control surface and the studied surface should be processed (machined) in the same way. The initial value (i) and the final value (f) of a property on both surfaces are measured within the relevant time period $\left(t_{r}\right)$. The result can be expressed as a ratio, an index, a percent, or an absolute value. The method is performed using the following steps:

Step 1. Selection of the studied surface $\left(\mathrm{S}_{\mathrm{s}}\right)$.

Step 2. Preparation of the control surface.

Step 3. Selection of control surface $\left(\mathrm{S}_{\mathrm{c}}\right)$.

Step 4. Selection of a property to be measured (p).

Step 5. Selection of replicate measurements (n).

Step 6. Identification of the relevant time period $\left(\mathrm{t}_{\mathrm{r}}\right)$ for measurement.

Step 7. Initial measurement of a property $\left(\mathrm{p}_{\mathrm{is}}\right)$ on $\mathrm{S}_{\mathrm{s}}$.

Step 8. Final measurement of a property $\left(\mathrm{p}_{\mathrm{fs}}\right)$ on $\mathrm{S}_{\mathrm{s}}$.

Step 9. Initial measurement of a property $\left(\mathrm{p}_{\mathrm{ic}}\right)$ on $\mathrm{S}_{\mathrm{c}}$.

Step 10. Final measurement of a property $\left(\mathrm{p}_{\mathrm{fc}}\right)$ on $\mathrm{S}_{\mathrm{c}}$.

Step 11. Evaluation of the measurements.

Step 12. Statistical analysis and interpretation of the results. 


\subsection{Results}

Contact angle and absorption of a liquid drop were used to develop the application of the inactivation method. Two measurements are shown, first with PF adhesive and then with water.

\subsubsection{Wood Surface Inactivation Tested with PF Adhesive}

Step 1. The $\mathrm{S}_{\mathrm{s}}$ was selected on the SP specimen, which was exposed to a high temperature under the procedure described in section 6.2.1. The area of the $S_{\mathrm{s}}$ was $20 \times 60 \mathrm{~mm}$.

Step 2. The $\mathrm{S}_{\mathrm{c}}$ surface was prepared by planing of a $1 \mathrm{~mm}$ thick surface layer from one side of the specimen.

Step 3. The $\mathrm{S}_{\mathrm{c}}$ with the area of $20 \times 60 \mathrm{~mm}$ was selected next to the $\mathrm{S}_{\mathrm{s}}$ (Figure 6.2).

Step 4. Two properties were measured: contact angle $(\theta)$ and absorption (AB) of a $5 \mu$ drop of PF adhesive. The same type of PF adhesive was used as in section 5.2.4. The adhesive properties are presented in Table 5.4. The contact angle was measured according to the approach discussed in section 4.3.2. The absorption was measured by evaluation of a side view area of the drop (Figure 6.3).
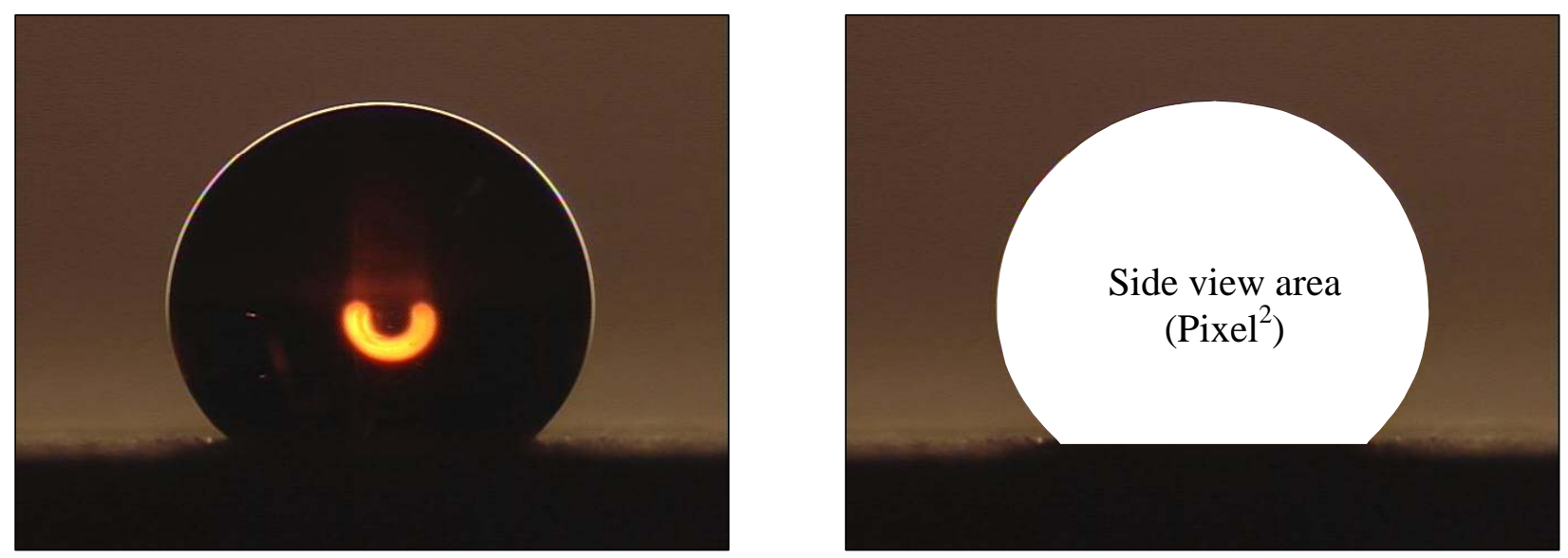

Figure 6.3. Actual image of a PF adhesive drop (left) and a side view area (right). 
It was assumed that the drop was symmetrical and that the volume of the drop was linearly proportional to the area. This relation is true when the drop is spherical. The ImagePro ${ }^{\mathrm{TM}}$ software measured the side view area. Since the magnification of the image varied between the replications (but not between initial and final image of the same measurement), the relative changes in the drop area $\left(\mathrm{AB}_{\% \mathrm{~s}}\right.$ and $\left.\mathrm{AB} \% \mathrm{c}\right)$ were used to quantify the adhesive absorption. The relative changes were expressed by percents.

Step 5. Twelve replicate measurements were done.

Step 6. The relevant time period was 120 seconds. The surface of a PF adhesive drop starts to consolidate after this period.

Step 7. The initial measurements of $\theta_{\text {is }}$ and $\mathrm{AB}_{\text {is }}$ were obtained immediately after the application of the adhesive drop to the $S_{\mathrm{s}}$.

Step 8. The final measurements of $\theta_{\mathrm{fs}}$ and $\mathrm{AB}_{\mathrm{fs}}$ were obtained 120 seconds after the application of the adhesive drop to the $S_{s}$.

Step 9. The initial measurements of $\theta_{\text {ic }}$ and $\mathrm{AB}_{\text {ic }}$ were obtained immediately after the application of the adhesive drop to the $S_{c}$.

Step 10. The final measurements of $\theta_{\mathrm{fc}}$ and $\mathrm{AB}_{\mathrm{fc}}$ were obtained 120 seconds after the application of the adhesive drop to the $S_{c}$. After this step, the measurements were summarized (Table 6.2). 
Table 6.2. Contact angle and absorption results for phenol-formaldehyde adhesive.

\begin{tabular}{|c|c|c|c|c|c|c|c|c|c|c|}
\hline \multirow{3}{*}{ Replication } & \multicolumn{10}{|c|}{ Phenol-formaldehyde Adhesive } \\
\hline & \multicolumn{4}{|c|}{ Contact Angle $\left(^{\circ}\right)$} & \multicolumn{6}{|c|}{ Absorption } \\
\hline & $\theta_{\text {is }}$ & $\theta_{\mathrm{fs}}$ & $\theta_{\text {ic }}$ & $\theta_{\mathrm{fc}}$ & $\mathrm{AB}_{\text {is }}$ & $\mathrm{AB}_{\mathrm{fs}}$ & $\mathrm{AB}_{\% \mathrm{~s}}$ & $\mathrm{AB}_{\mathrm{ic}}$ & $\mathrm{AB}_{\mathrm{fc}}$ & $\mathrm{AB}_{\% \mathrm{c}}$ \\
\hline 1 & 112.4 & 100.3 & 93.3 & 81.7 & 142.8 & 128.5 & 10.0 & 166.5 & 142.4 & 14.5 \\
\hline 2 & 122.1 & 105.2 & 85.5 & 79.1 & 151.3 & 138.7 & 8.3 & 187.8 & 165.5 & 11.9 \\
\hline 3 & 113.5 & 105.2 & 98.8 & 87.9 & 137.6 & 125.2 & 9.0 & 212.3 & 186.6 & 12.1 \\
\hline 4 & 109.3 & 89.9 & 104.4 & 100.2 & 143.8 & 132.6 & 7.8 & 179.3 & 161.3 & 10.0 \\
\hline 5 & 101.1 & 90.2 & 91.1 & 78.7 & 175.9 & 157.1 & 10.7 & 209.3 & 184.7 & 11.7 \\
\hline 6 & 108.9 & 96.3 & 106.9 & 100.3 & 138.7 & 128.4 & 7.4 & 178.5 & 160.2 & 10.3 \\
\hline 7 & 112.9 & 90.5 & 91.7 & 86.1 & 167.1 & 149.5 & 10.5 & 210.6 & 186.4 & 11.5 \\
\hline 8 & 101.7 & 82.4 & 94.7 & 80.8 & 159.9 & 141.4 & 11.6 & 174.5 & 151.4 & 13.2 \\
\hline 9 & 115.1 & 102.0 & 88.3 & 75.5 & 156.9 & 139.7 & 10.9 & 158.3 & 139.0 & 12.2 \\
\hline 10 & 97.3 & 78.0 & 89.3 & 78.8 & 152.1 & 132.6 & 12.9 & 162.7 & 137.8 & 15.3 \\
\hline 11 & 91.6 & 86.0 & 103.3 & 86.9 & 135.4 & 124.0 & 8.4 & 162.5 & 144.0 & 11.4 \\
\hline 12 & 110.8 & 88.1 & 81.3 & 75.5 & 176.6 & 157.1 & 11.0 & 163.9 & 144.1 & 12.1 \\
\hline Average & 108.1 & 92.8 & 94.1 & 84.3 & 153.2 & 137.4 & 9.9 & 180.5 & 158.6 & 12.2 \\
\hline
\end{tabular}

Step 11. The measurements were evaluated as an inactivation ratio (IR) and an absorption index (ABI). The inactivation ratio was defined as the ratio between the contact angle of the studied surface $\left(\theta_{\mathrm{s}}\right)$ and the control surface $\left(\theta_{\mathrm{c}}\right)$ :

$$
I R=\frac{\theta_{s}}{\theta_{c}}
$$

Equation 6.1

The initial $\mathrm{IR}_{\mathrm{i}}$ and the final $\mathrm{IR}_{\mathrm{f}}$ were calculated. The result was expressed as an average value of the $\mathrm{IR}_{\mathrm{i}}$ and the $\mathrm{IR}_{\mathrm{f}}$. The absorption index was calculated as:

$$
A B I=\frac{A B_{\% s}}{A B_{\% c}}
$$

Equation 6.2

Step 12. Statistical t-test was used to indicate significant difference in a measured property obtained on the studied and control surfaces. The confidence level was 95\%. An example of statistical analysis as generated by statistical program STATGRAF is shown in Table 6.3. Statistically significant differences were found for all comparisons in a property (i.e., contact and absorption) obtained on the studied and control surfaces. The calculated average IR was 1.14 and the $\mathrm{ABI}$ was 0.81. Interpretation of IR and $\mathrm{ABI}$ is given in section 6.5. 
Table 6.3. Two-Sample analysis results (t-test).

\begin{tabular}{|c|c|c|c|c|}
\hline \multirow{4}{*}{ Sample Statistics: } & & $\mathrm{PF} \quad \theta_{\mathrm{fs}}$ & $\mathrm{PF} \quad \theta_{\mathrm{fc}}$ & Pooled \\
\hline & Number of Obs. & 12 & 12 & 24 \\
\hline & Average & 92.8417 & 84.2917 & 88.5667 \\
\hline & Variance & 79.6936 & 72.2099 & 75.9517 \\
\hline & Std. Deviation & 8.92712 & 8.49764 & 8.7150 \\
\hline Conf. Interval For & Diff. in Means: & Per & & \\
\hline Hypothesis Test for & $\begin{aligned} \text { HO: Diff } & =0 \\
\text { vs Alt } & : \mathrm{NE} \\
\text { at Alpha } & =0.05\end{aligned}$ & $\begin{array}{l}\text { Computed } \\
\text { Sig. Leve } \\
\text { So reject }\end{array}$ & $\begin{array}{l}\text { statistic }= \\
=0.0251277 \\
\text { o. }\end{array}$ & 2.40311 \\
\hline
\end{tabular}

\subsubsection{Wood Surface Inactivation Tested with Water}

The specimens from the previous section were also tested with water by using the procedure explained in section 6.3. Therefore, the first five steps were the same, except that $2.5 \mu 1$ of distilled water was applied instead of $5 \mu 1$.

Step 6. The relevant time period was presented with the time needed for the complete absorption of a water drop into the control surface. This time varied, which is shown in (Table 6.4). On average, a water drop was completely absorbed into the $S_{c}$ after 215 seconds.

Step 7. The initial measurements of $\theta_{\text {is }}$ and $\mathrm{AB}_{\text {is }}$ were obtained immediately after the application of the water drop to the $S_{\mathrm{s}}$.

Step 8. The final measurements of $\theta_{\mathrm{fs}}$ and $\mathrm{AB}_{\mathrm{fs}}$ were obtained after the $\mathrm{t}_{\mathrm{r}}$.

Step 9. The initial measurement of $\theta_{\text {ic }}$ was obtained immediately after the application of the water drop to the $S_{c}$. $A_{\text {ic }}$ was not measured by the image analysis because the water drop was completely absorbed during the observation. This meant that the $\mathrm{AB} \%$ was $100 \%$.

Step 10. The final measurement of $\theta_{\mathrm{fc}}$ and $\mathrm{AB}_{\mathrm{fc}}$ were obtained after the $\mathrm{t}_{\mathrm{r}}$. The measurements were then summarized (Table 6.4). 
Table 6.4. Contact angle and absorption of water.

\begin{tabular}{|c|c|c|c|c|c|c|c|c|c|}
\hline \multirow{3}{*}{ Replication } & \multicolumn{9}{|c|}{ Water } \\
\hline & \multirow{2}{*}{$\begin{array}{l}\mathbf{t}_{\mathbf{r}} \\
\mathrm{s} .\end{array}$} & \multicolumn{4}{|c|}{ Contact Angle } & \multicolumn{4}{|c|}{ Absorption } \\
\hline & & $\theta_{\text {is }}$ & $\theta_{\mathrm{fs}}$ & $\theta_{\text {ic }}$ & $\theta_{\mathrm{fc}}$ & $\mathrm{AB}_{\text {is }}$ & $\mathrm{AB}_{\mathrm{fs}}$ & $\mathrm{AB}_{\% \mathrm{~s}}$ & $\mathrm{AB}_{\% c}$ \\
\hline 1 & 190 & 103.5 & 77.8 & 39.4 & 0.0 & 66.1 & 48.5 & 26.7 & 100.0 \\
\hline 2 & 220 & 91.9 & 73.5 & 41.2 & 0.0 & 58.7 & 39.9 & 32.0 & 100.0 \\
\hline 3 & 195 & 92.1 & 74.9 & 38.7 & 0.0 & 81.0 & 55.8 & 31.0 & 100.0 \\
\hline 4 & 225 & 90.2 & 73.2 & 52.8 & 0.0 & 73.6 & 50.2 & 31.8 & 100.0 \\
\hline 5 & 210 & 92.3 & 76.9 & 52.9 & 0.0 & 105.2 & 82.2 & 21.8 & 100.0 \\
\hline 6 & 220 & 91.7 & 59.8 & 54.2 & 0.0 & 78.5 & 60.8 & 22.6 & 100.0 \\
\hline 7 & 250 & 95.6 & 61.7 & 54.4 & 0.0 & 84.6 & 63.6 & 24.8 & 100.0 \\
\hline 8 & 220 & 98.7 & 66.1 & 49.9 & 0.0 & 77.4 & 54.6 & 29.4 & 100.0 \\
\hline 9 & 225 & 90.2 & 70.3 & 55.1 & 0.0 & 68.9 & 47.0 & 31.8 & 100.0 \\
\hline 10 & 245 & 90.2 & 65.2 & 59.2 & 0.0 & 67.6 & 44.3 & 34.4 & 100.0 \\
\hline 11 & 180 & 92.8 & 79.6 & 41.4 & 0.0 & 102.9 & 81.7 & 20.6 & 100.0 \\
\hline 12 & 200 & 92.6 & 79.1 & 47.3 & 0.0 & 66.9 & 48.6 & 27.4 & 100.0 \\
\hline Average & 215 & 93.5 & 71.5 & 48.9 & 0.0 & 77.6 & 56.4 & 27.9 & 100.0 \\
\hline
\end{tabular}

Step 11. The measurements were evaluated as IR and ABI by using Eq. 6.1 and Eq. 6.2.

Step 12. Statistically significant differences were found for all comparisons in a property (i.e. contact and absorption) obtained on the studied and control surfaces. The $\mathrm{IR}_{\mathrm{i}}$ was 1.91 and the $\mathrm{ABI}$ was 0.28 . Interpretation of IR and $\mathrm{ABI}$ is given in section 6.5.

\subsubsection{Adhesive Bond Performance}

Descriptive statistics of the maximum and arrested values of the strain energy release rate (SERR) of the samples bonded with PF adhesive are shown in Table 6.5.

Table 6.5. SERR $\left(\mathrm{J} / \mathrm{m}^{2}\right)$ of samples bonded with PF adhesive.

\begin{tabular}{|c|c|c|c|c|}
\hline \multirow[t]{2}{*}{ Southern Pine } & \multicolumn{2}{|c|}{ Inactivated Surface } & \multicolumn{2}{|c|}{ Control Surface } \\
\hline & $\mathbf{G}_{\max }$ & $\mathbf{G}_{\text {arr }}$ & $\mathbf{G}_{\max }$ & $\mathbf{G}_{\text {arr }}$ \\
\hline AVERAGE & 20.0 & 16.0 & 188.8 & 171.0 \\
\hline STDEV & 4.6 & 3.7 & 35.7 & 38.9 \\
\hline $\operatorname{COV}(\%)$ & 23.1 & 23.0 & 18.9 & 22.7 \\
\hline
\end{tabular}




\subsection{Discussion}

The surface inactivation method, either when using PF adhesive or water results, was able to detect wood surface inactivation. However, the results were less pronounced when using the adhesive. The results were related to the adhesion results, which were evaluated by fracture mechanics testing. Even though the change in adhesion was several times greater than the relative changes in the IR or ABI obtained by the surface inactivation method, the latter was sufficient to detect inactivated wood surfaces. The difference between the property obtained on the studied (inactivated) surfaces and on the control (fresh) surfaces were consistently statistically significant.

When the results obtained with $P F$ adhesives were compared with $G_{\max }$, the analysis showed that the IR of 1.14 and the ABI of 0.81 presented severely inactivated wood surfaces. The $G_{\max }$ of surfaces bonded with $P F$ adhesive was only $20 \mathrm{~J} / \mathrm{m}^{2}$, while control surfaces exhibited $\mathrm{G}_{\max }$ of $188.8 \mathrm{~J} / \mathrm{m}^{2}$. The IR of 1.14 indicates that the contact angle of the studied wood surfaces was on average $14 \%$ higher than the contact angle of the control surfaces. A higher contact angle presented a lower surface wettability. The ABI of 0.81 indicated that the inactivated surfaces had only $81 \%$ of the absorption capacity for the PF adhesive in comparison with the control surfaces. Thus the reduced wettability and absorbtivity of the surface by PF adhesive resulted in weak adhesion.

The results obtained with water showed an even more distinct relationship between adhesion and $I R$ (or $A B I$ ). The $I_{i}$ was 1.91 and the $A B I$ was 0.28 . The $I_{i}$ of 1.91 indicates that the initial contact angle of the inactivated wood surface was on average $91 \%$ higher than the initial contact angle of the control surface. The ABI of 0.28 indicates that the inactivated surface had only $28 \%$ of the absorption capacity for water in comparison with the control surface. The interpretation of surface inactivation by using ABI and IR is shown in Figure 6.4. 


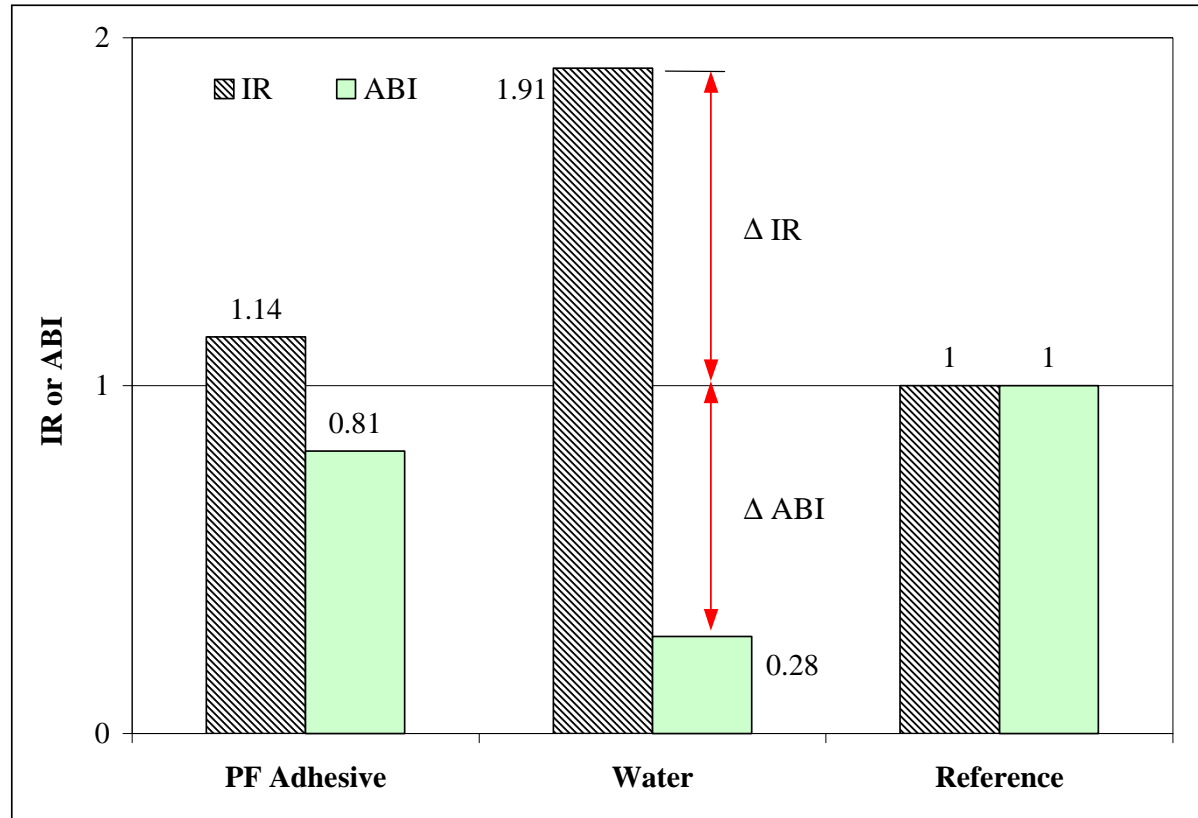

Figure 6.4. PF adhesive and water were used as test liquids to evaluate SP surface inactivation by using inactivation ratio and absorption index. The greater the deviation (i.e., $\triangle I R$ or $\triangle A B I$ ) from 1, the more severe the surface inactivation.

Again, the results on IR and ABI did not scale on the same range as the relative change in $\mathrm{G}_{\max }$ between inactivated and control surfaces. The method did not provide information on the nature of the relationship among adhesion, inactivation ratio, and absorption index. For that purpose, samples with different severities of inactivation should be prepared and tested. A plot of $\mathrm{G}_{\max }$ against IR or ABI may provide evidence of the interdependence. In spite of the lack of this relationship, the indication of severe surface inactivation was evident from these results. The method is simple and fast, which makes it feasible in an industrial environment.

\subsection{Conclusions}

The surface inactivation method for the detection of an inactivated wood surface is simple and useful. It distinguishes between inactivated and fresh wood surfaces prior to bonding based on wettability and absorption measurements. The outcome followed the results on adhesion, but the range of the relative changes was different. The method is more sensitive when water is used as a test liquid. 


\section{Chapter 7. Summary and Conclusions}

\subsection{Summary}

This study dealt with heat-induced wood surface inactivation of yellow-polar and southern pine. The main objective of the study was identification of temperature and time exposure levels that cause wood surface inactivation for these two wood species. Additionally, chemical and physical characterization of wood surfaces in regard to inactivation was accomplished. Surface chemistry and wettability were evaluated by X-ray photoelectron spectroscopy (XPS) and liquid contact angle by means of the sessile drop technique. Bond performance was determined by fracture testing using two adhesive systems. Later, chemical treatment methods of reactivation were used to improve adhesion of inactivated wood surfaces. Finally, a simple comparative method was developed for the rapid identification of inactivated wood surfaces.

The results showed that experimental observation on surface chemistry of wood constituents corresponded to the theoretical interpretation. Cellulose had the highest value of the oxygen to carbon $(\mathrm{O} / \mathrm{C})$ ratio, followed by lignin, yellow-poplar extractives, and southern pine extractives. The $\mathrm{C} 1 / \mathrm{C} 2$ ratio increased in the opposite order. The $\mathrm{C} 1$ component presents carbon, which is bonded to another carbon or hydrogen atom. The $\mathrm{C} 2$ component is carbon in $\mathrm{C}-\mathrm{O}$ bond. A high $\mathrm{O} / \mathrm{C}$ ratio or a low $\mathrm{C} 1 / \mathrm{C} 2$ ratio presented a wood surface containing mostly polysaccharides, while a low $\mathrm{O} / \mathrm{C}$ ratio and a high $\mathrm{C} 1 / \mathrm{C} 2$ ratio reflected a high concentration of non-polar organic compounds with significant mobility; i.e., extractives, degraded VOCs, and possibly lignin on the wood surface. The removal of the extractives increased the $\mathrm{O} / \mathrm{C}$ ratio and decreased the $\mathrm{C} 1 / \mathrm{C} 2$ ratio of the wood surface. The assignment of the carbon $\mathrm{C} 1 \mathrm{~s}$ peak to extractives, VOCs, and lignin cannot be distinguished by XPS analysis. However, since lignin is relatively immobile, and solvent treatment reduced the $\mathrm{C} 1$ atomic percent, the increased $\mathrm{C} 1 / \mathrm{C} 2$ ratio was likely the result of extractive/VOCs migration to the surface and residual products of the VOCs pyrolysis, which remained connected to the surface. 
The water contact angle observed on the wood surface decreased with time; an equilibrium was never reached. Southern pine exhibited a higher contact angle than yellowpoplar regardless of the temperature exposure. The extraction with acetone-water, which followed wood drying, improved wettability for both wood species. The extraction of the samples prior to drying did not improve wettability. This suggests that changes in surface energetics are related not only to extractives content but also to other factors, such as partial VOCs deposition on the wood surface. Wettability of the wood surface increased with the O/C ratio and it decreased with the $\mathrm{C} 1 / \mathrm{C} 2$ ratio.

The strain energy release rate obtained by the fracture test showed that southern pine was more susceptible to surface inactivation than yellow-poplar. Adhesive bond performance of southern pine dropped by a factor of two for samples exposed to high temperature. From a mechanical standpoint, the southern pine surface was inactive for PF adhesive when dried at $156^{\circ} \mathrm{C}$ or higher, and for PVA adhesives when dried at $187^{\circ} \mathrm{C}$. Yellow-poplar surfaces did not show a significant inactivation phenomenon when exposed to drying temperatures up to $187^{\circ} \mathrm{C}$. These specimens exhibited higher adhesive bond performance than southern pine specimens regardless of the drying temperature or adhesive used.

Wood surface chemistry changed in regard to drying temperature. The oxygen to carbon ratio $(\mathrm{O} / \mathrm{C})$ decreased, and the $\mathrm{C} 1 / \mathrm{C} 2$ ratio increased with temperature. Both yellow-poplar and southern pine surfaces indicated higher extractives contents, lignin content, and perhaps adsorbed VOCs, for samples exposed to higher temperatures, which modified the wood surface from hydrophilic to hydrophobic.

Since the hydrophobic wood surface repelled water, wettability of this surface was low (i.e., a high contact angle). The highest contact angle was obtained on the surfaces that were exposed to the highest drying temperature. The contact angle increased with drying temperature and decreased with contact angle measurement time. Wood species affected wettability, whereby southern pine exhibited higher contact angles than yellow-poplar at all studied temperature exposures. Inactivation, as indicated by a high contact angle, occurred at a lower surface temperature during drying for southern pine than yellow-poplar. Wettability was crucial for good 
adhesion. The highest values of the $G_{\max }$ were obtained at high $\cos \theta$, (i.e., low contact angle), which presents good wettability. $\mathrm{G}_{\max }$ increased with $\cos \theta$, regardless of wood species.

Several chemical treatments improved the wettability of inactivated wood surfaces. Wettability of the treated surfaces does not necessarily correlate with adhesion, especially when evaluated with a liquid, which was not used for bonding. This suggests that the wettability should be evaluated by a contact angle measurement using the adhesive. The critical surface tension of an inactivated wood surface was lower than that of a fresh wood surface reported in the literature.

Attempts to reverse surface inactivation involved aqueous solutions of xylanase, sodium hydroxide $(\mathrm{NaOH})$, xylanase- $\mathrm{NaOH}$, and hydroxymethylated resorcinol (HMR). Adhesion improvement due to surface chemical treatment was not evident for specimens bonded with PVA. Enzymatic treatment with xylanases did not improve adhesion. The HMR coupling agent was not operative on inactivated surfaces bonded with PF adhesive. $\mathrm{NaOH}$ was the most effective in restoring bonding ability of PF adhesive with inactivated wood surfaces. The maximum strain energy release rate $\left(\mathrm{G}_{\max }\right)$ of specimens treated with $\mathrm{NaOH}$ increased by a factor of three when compared with inactivated specimens. Of the chemical treatments employed by this study, $\mathrm{NaOH}$ was the most effective for improving adhesion, while HMR had the greatest influence on improving water wettability.

The choice of the adhesive drastically impacted the adhesion of inactivated wood assemblies. The inclusion of HMR coupling agent into the PF adhesive mixture was unsuccessful in restoring the adhesion of inactivated wood surfaces. PMDI adhesive provided a three times higher $G_{\max }$ than PF adhesive. Since this effect was similar to the effect of surface treatment with $\mathrm{NaOH}$, the remedy for wood surface inactivation should be based on the usage of the adhesive with a better performance.

The surface inactivation method for the detection of an inactivated wood surface is simple and useful. It distinguishes between inactivated and fresh wood surfaces prior to bonding based on wettability and absorption measurements. It might be possible to install in-line testing hardware to diagnose surface inactivation in real time. 


\subsection{Final Conclusions}

The comparative analysis of inactivated surfaces revealed clear relationships between wood surface chemistry, wettability, and adhesive bond performance. Extractives migration and VOCs degradation obviously play a significant role in heat-induced surface inactivation of southern pines.

Solvent extraction after drying improves wettability, whereas, extraction prior to drying is less effective. Wettability is directly related to the $\mathrm{O} / \mathrm{C}$ ratio and inversely related to the $\mathrm{C} 1 / \mathrm{C} 2$ ratio, suggesting that increased concentration of non-polar substances; i.e., extractives and VOCs on a wood surface reduces wettability. Southern pine clearly has a lower wettability than yellowpoplar, which the comparison of XPS and solvent extraction results indicate is due to a greater concentration of extractives and degraded VOCs on the surface.

Inactivation, as indicated by a high contact angle, occurs at a lower surface temperature during the drying of southern pine (about $150^{\circ} \mathrm{C}$ ) than yellow-poplar (about $170^{\circ} \mathrm{C}$ ). Adhesive bond performance, as determined by fracture mechanics testing, improves when contact angle decreases $\left(\theta_{\mathrm{i}}<90^{\circ}\right)$. Bond performance of PVA adhesive is less affected by drying temperature than PF adhesive, at least with the adhesive formulations used in this research. In terms of adhesion, southern pine is susceptible to inactivation at temperatures above $156^{\circ} \mathrm{C}$. Yellowpoplar does not show a significant surface inactivation for the investigated temperature range.

Of the chemical treatments employed in this study, $\mathrm{NaOH}$ is the most effective for improving adhesion, while HMR has the greatest influence on improving water wettability. PMDI adhesive significantly increased fracture energy of bonded inactivated wood surfaces. However, the maximum improvement in adhesion, caused by surface treatment or by exchange of the adhesive mixture, approaches only $75 \%$ of the adhesion that is established when bonding fresh wood surfaces. 


\section{References}

1. Adamson, A.W. 1990. Physical Chemistry of Surface. Fifth Edition. John Wiley \& Sons, Inc. New York, 777 p.

2. Ahmed, A., A. Adnot, and S. Kaliaguine. 1987. ESCA Study of the solid residues of supercritical extraction of populus tremuloides in methanol. Journal of Applied Polymer Science 34:359-375.

3. Alén R., E. Kuoppala, and P. Oesch. 1996. Formation of the main degradation compound groups from wood and its components during pyrolysis. Journal of Analytical and Applied Pyrolysis 36:137-148.

4. ASTM D 3433-93. 1997. Standard test method for fracture strength in cleavage of adhesives in bonded metal joint. Pages 212-218 in Annual Book of ASTM Standards, V 15.06 Adhesives. ASTM.

5. Baier, R.E., E.G. Shafrin, and W.A. Zisman. 1968. Adhesion: Mechanisms that assist or impede it. Science, New Series, 162 (3860):1360-1368.

6. Banerjee, S. 2001. Mechanisms of terpene release during sawdust and flake drying. Holzforschung 55(4):413-416.

7. Banerjee, S., W. Su, P. Wild, L.P. Otwell, M.E. Hittmeier, and K. Nichols. 1998. Wet line extension reduces VOCs from softwood drying. Environmental Science \& Technology 32(9):1303-1307.

8. Barry, A.O., Z. Koran, and Kaliaguine, S. 1990. Surface analysis by ESCA of sulfite post-treated CTMP. Journal of Applied Polymer Science 39:31-42.

9. Bateup, B.O. 1981. Surface chemistry and adhesion. International Journal of Adhesion and Adhesives 1:233-239.

10. Baumann, M.G.D., S.A. Batterman, and G-Z. Zhang. 1999. Terpene emissions from particleboard and medium-density fiberboard products. Forest Products Journal 49(1):4956.

11. Beamson, G. and Briggs, D. 1992. High Resolution XPS of Organic Polymers. Wiley, Chichester, UK, 295 p. 
12. Ben, Y., B.V. Kokta, J. Doucet, and S. Kaliaguine. 1993. Effect of chemical pretreatment on chemical characteristics of steam explosion pulps of Aspen. Journal of Wood Chemistry and Technology 13(3):349-369.

13. Birdi, K.S. 1997. Handbook of Surface and Colloid Chemistry. CRC Press. Boca Raton, New York, 763 p.

14. Blackman, B., J.P. Dear, A.J. Kinloch, and S. Osiyemi. 1991. The calculation of adhesive fracture energies from double-cantilever beam test specimens. Journal of Materials Science Letters 10:253-256.

15. Bodig, J. 1962. Wettability related to gluabilities of five Philippine mahoganies. Forest Products Journal 12(6):265-270.

16. Bodig, J. and B.A. Jayne. 1982. Mechanics of Wood and Wood Composites. Van Nostrand, New York, 712 p.

17. Bohlen, J.C. 1972. Shear strength of high-temperature heat-treated lumber laminated with phenol-resorcinol adhesives. Forest Product Journal 22(12) 17-24.

18. Börås, L. and P. Gatenholm. 1999. Surface composition and morphology of CTMP Fibers. Holzforschung 53(2):188-194.

19. Briggs, D. and M.P. Seah. 1990. Practical Surface Analysis. Volume 1. Auger and X-ray Photoelectron Spectroscopy. John Wiley \& Sons, Chichester, UK, 657 p.

20. Brune, D., R. Hellborg, H.J. Whitlow, and O. Hunderi. 1997. Surface Characterization. Wiley-VCH, New York, 291 p.

21. Carpenter, M.W. 1999. Characterizing the Chemistry of Yellow-poplar Surfaces Exposed to Different Surface Energy Environments Using DCA, DSC and XPS (Master's Thesis). Morgantown (WV): West Virginia University, $182 \mathrm{p}$.

22. Chen, C-M. 1970. Effect of extractive removal on adhesion and wettability of some tropical woods. Forest Products Journal 20(1):36-40.

23. Chow, S.Z. 1971. Determining veneer surface inactivation by a reflectance colorimeter. Forest Product Journal 21(2):19-24.

24. Chow, S. 1975. Minimizing wood surface inactivation at high temperatures by boron compounds. Forest Products Journal 25(5):41-48. 
25. Christiansen, A.W. 1990. How overdrying wood reduces its bonding to phenolformaldehyde adhesives: A critical review of the literature. Part I. Physical responses. Wood and Fiber Science 22(4):441-459.

26. Christiansen, A.W. 1991. How overdrying wood reduces its bonding to phenolformaldehyde adhesives: A critical review of the literature. Part II. Chemical reactions. Wood and Fiber Science 23(1):69-84.

27. Collett, B.M. 1972. A review of surface and interfacial adhesion in wood science and related fields. Wood Science and Technology 6:1-42.

28. Currier, R.A. 1958. High drying temperatures- Do they harm veneer? Forest Products Journal 8(4):128-136.

29. Davis, M., B. Rosin, L.L. Landucci, and T.W. Jeffries. 1997. Characterization of UV absorbing products released from kraft pulps by xylanases. Biological Sciences Symposium, San Francisco. Tappi Press 435-442.

30. Doris, G.M. and D.G. Gray 1978a. The surface analysis of paper and wood fibers by ESCA. I. Cellulose Chemistry and Technology 12:9-23.

31. Doris, G.M. and D.G. Gray 1978b. The surface analysis of paper and wood fibers by ESCA. II. Surface composition of mechanical pulps. Cellulose Chemistry and Technology 12:721-734.

32. Ebewele, R.O., B. River, and J. Koutsky. 1979. Tapered double cantilever beam fracture tests of phenolic-wood adhesive joint. Part I. Development of specimen geometry; effects of bondline thickness, wood anisotropy and cure time on fracture energy. Wood and Fiber Science 11(3):197-213.

33. Ebewele, R.O., B. River, and J. Koutsky. 1980. Tapered double cantilever beam fracture tests of phenolic-wood adhesive joint. Part II. Effects of surface roughness, the nature of surface roughness, and surface aging on joint fracture energy. Wood and Fiber Science 12(1):40-65.

34. Evans, F.D. and H. Wennerström. 1999. The Colloidal Domain. Where Physics, Chemistry, Biology, and Technology Meet. Second Edition. Wiley-VCH, 632 p. 
35. Fengel, D. and G. Wegener. 1989. Wood. Chemistry, Ultrastructure, Reactions. Walter de Gruyter, Berlin, 613 p.

36. Forbes, C. 1998. Wood surface inactivation and adhesive bonding. Wood product notes. http://www.ces.ncsu.edu/nreos/wood/wpn/wood_surface.htm.

37. Frazier, C.E., M.P. Laborie, and J.M. Gagliano. 2000. Fracture cleavage testing of adhesively-bonded wood. In Evans P.D. compiler: $5^{\text {th }}$ Pacific Rim Bio-Based Composite Symposium, Proceedings, Canberra, Australia, 2000, 358-364.

38. Freeman, H.A. 1959. Properties of wood and adhesion. Forest Products Journal 9(12):451-458.

39. Gagliano, J.M. 2001. An Improved Method for the Fracture Cleavage Testing of Adhesively-Bonded Wood (Master's Thesis). Blacksburg (VA): Virginia Polytechnic Institute and State University. $87 \mathrm{p}$.

40. Gagliano, J.M. and C.E. Frazier. 2001. Improvements in the fracture cleavage testing of adhesively-bonded wood. Wood and Fiber Science 33(3):377-385.

41. Garcia, O., A.L. Torres, J.F. Colom, F.I.J. Pastor, P. Diaz, and T. Vidal. 2001. Effect of cellulase-assisted refining on the properties of dried and never-dried Eucalyptus pulp. Cellulose (In press).

42. Gardner, D.J. and T.J. Elder. 1988. Surface activation treatment of wood and its effect on the gel time of phenol-formaldehyde resin. Wood and Fiber Science 20(3):378-385.

43. Gardner, D.J. and T.J. Elder. 1990. Bonding surface activated hardwood flakeboard with phenol-formaldehyde resin. I. Physical and mechanical properties. Holzforschung 44(3):201-206.

44. Gardner, D.J., N.C. Generalla, D.W. Gunnells, and M.P. Wolcott. 1991a. Dynamic Wettability of Wood. Langmuir 7:2498-2502.

45. Gardner, D.J., J.G. Ostmeyer, and T.J. Elder. 1991b. Bonding surface activated hardwood flakeboard with phenol-formaldehyde resin. II. Flake surface chemistry. Holzforschung 44(3):215-222.

46. Gardner, D.J., M.P. Wolcott, L. Wilson, Y. Huang, and M. Carpenter. 1995. Our understanding of wood surface chemistry in 1995. Pages 29-36 In: Christiansen, A.W. 
and A.H. Conne, editors. Wood Adhesives 1995. Proceedings of a symposium sponsored by USDA. Proceedings No. 7296. FPS, Madison, WI.

47. Gardner, D.J., W.T. Tze, and S.Q. Shi. 2000. Adhesive wettability of hydroxymethyl resorcinol (HMR) treated wood. Pages 65-67 in Wood Adhesive 2000. Extended Abstracts. Forest Product Society, Lake Tahoe.

48. Garnier, G. and W.G. Glasser. 1994. Measurement of surface free energy of amorphous cellulose by alkane adsorption: A critical evaluation of inverse gas chromatography (IGC). Journal of Adhesion 46:165-180.

49. Gauthier, M.M. 1995. Adhesives and Sealants. Pages 633-638 in: Brinson, H.F., editor. ASM Engineered Materials Handbook. Adhesives. ASM International.

50. Gindl, M., G. Sinn, W. Gindl, A. Reiterer, and S. Tschegg. 2001. A comparison of different methods to calculate the surface free energy of wood using contact angle measurements. Colloids and Surfaces 181:279-287.

51. Glasser, W.G., V. Dave, and C.E. Frazier. 1993. Molecular weight distribution of (semi-) commercial lignin derivatives. Journal of Wood Chemistry and Technology 13(4):545559.

52. Glasser, W.G. 2000. Classification of lignin according to chemical and molecular structure. Pages 216-238 In: Lignin: Historical, Biological, and Materials Perspectives. American Chemical Society, Symposium Series 742, Washington, DC.

53. Gray, V.R. 1962. The Wettability of Wood. Forest Products Journal 12(9):452-461.

54. Gunnells, D.W., D.J. Gardner, and M.P. Wolcott. 1994. Temperature dependence of wood surface energy. Wood and Fiber Science 26(4):447-455.

55. Gupta, S., B. Bhushan, and G.S. Hoondal. 2000. Isolation, purification and characterization of xylanase from Staphylococcus sp. SG-13 and its application in biobleaching of kraft pulp. Journal of Applied Microbiology 88:325-334.

56. Hagstrum, H.D. 1972. Electronic characterization of solid surfaces. Science, New Series 178, (4058):275-282.

57. Hancock, W.V. 1963. Effect of heat treatment on the surface of Douglas-fir veneer. Forest Product Journal 13(2):81-88. 
58. Hashemi, S., A.J. Kinloch, and J.G. Williams. 1990. The analysis of interlaminar fracture in uniaxial fiber-polymer composites. Proceedings of Royal Society of London, Series A, 427(1872):173-199.

59. Haskell. H.H., W.M. Bair, and W. Donaldson. 1966. Progress and problems in the southern pine plywood industry. Forest Products Journal 16(4):19-24.

60. Haupt, R.A. and T. Sellers Jr. 1994. Phenolic resin-wood interaction. Forest Products Journal 44(2):69-73.

61. Haygreen, J.G. and J.L. Bowyer. 1996. Forest Products and Wood Science: An Introduction. 3rd Edition. Ames: Iowa State University Press, 484 p.

62. Helm, R.F. 2002. Personal communication.

63. Hemingway, R.W. 1969. Thermal instability of fats relative to surface wettability of yellow birchwood (Betula lutea). Tappi 52(11):2149-2155.

64. Hiemenz, P.C. and R. Rajagopalan. 1997. Principles of Colloid and Surface Chemistry. Third Edition. Marcel Dekker, Inc. New York, 650 p.

65. Hillis, W.E. 1962. Wood Extractives and their Significance to the Pulp and Paper Industry. Academic Press, New York and London, 513 p.

66. Hillis, W.E. 1984. High temperature and chemical effects on wood stability. Wood Science and Technology 18:281-293.

67. Hillis, W.E. 1987. Heartwood and Tree Exudates. Springer-Verlag, Berlin, 268 p.

68. Ho F.F.-L. 1982. ESCA analysis of functional groups on modified polymer surfaces. Pages 105-133 in: Windawi, H. and F.F.-L. Ho. Applied Electron Spectroscopy for Chemical Analysis. John Wiley \& Sons, New York.

69. Hollander, J.M., D.A., Shirley, and B.P. Stoicheff. 1981. The 1981 Nobel Prize in Physics. Science, New Series, 214(4521):629-633.

70. Hon, D.N.-S. 1984. ESCA study of oxidized wood surfaces. Journal of Applied Polymer Science 29:2777-2784.

71. Hse, C. Y. 1972. Wettability of southern pine veneer by phenol formaldehyde wood adhesives. Forest Products Journal 22(1):51-56. 
72. Hse, C.Y. and M. Kuo. 1988. Influence of extractives on wood gluing and finishing-a review. Forest Product Journal 38(1):52-56.

73. Hua, X., S. Kaliaguine, B.V. Kokta, and A. Adnot. 1993a. Surface analysis of explosion Pulps by ESCA. Part 1. Carbon (1s) spectra and oxygen-to-carbon ratios. Wood Science and Technology 27:449-459.

74. Hua, X., S. Kaliaguine, B.V. Kokta, and A. Adnot. 1993b. Surface analysis of explosion Pulps by ESCA. Part 2. Oxygen (1s) and sulfur (2p) spectra. Wood Science and Technology 28:1-8.

75. Istone, W.K. 1995. X-Ray Photoelectron Spectroscopy (XPS). Pages 235-268 in: Conners, T.E. and S. Banerjee, editors. Surface Analysis of Paper. CRC Press, Inc. Boca Raton, Florida.

76. Jamin, S.A. 2001. Personal communications.

77. Jeffries, T.W. and L. Viikari. 1996. Enzymes for Pulp and Paper Processing. American Chemical Society, Washington, D.C., 326 p.

78. Johnson, R.A.; Bhattacharyya, G. K. 1992. Statistics, Principles and Methods. Second Edition. John Wiley \& Sons, Inc. New York, 686 p.

79. Johnson, S.E. and F.A. Kamke. 1992. Quantitative analysis of gross adhesive penetration in wood using fluorescence microscopy. Journal of Adhesion 40:47-61.

80. Kadlec, K.M. 1980. Predicting Surface Inactivation after Platen Drying of SecondGrowth Douglas-Fir Veneer (Master's Theses). Oregon State University, 1-63.

81. Kajita, H. and C. Skaar. 1992. Wettability of the surfaces of some American softwoods. Mokuzai Gakkaishi 38(5):516-521.

82. Kaldas, M.L., P.A. Cooper, and R. Sodhi. 1998. Oxidation of wood components during chromated copper arsenate (CCA-C) fixation. Journal of Wood Chemistry and Technology 18(1):53-67.

83. Kamdem, D.P., B. Riedel, A. Adnot, and S. Kaliaguine. 1991. ESCA spectroscopy of poly(methyl methacrylate) grafted onto wood fibers. Journal of Applied Polymer Science 43:1901-1912. 
84. Kato, K.L. and R.E. Cameron. 1999. A review of the relationship between thermallyaccelerated aging of paper and hornification. Cellulose 6:23-40.

85. Kazayawoko, M., J.J. Balatinecz, R.T. Woodhams, and R.N.S. Sodhi. 1998. X-ray photoelectron spectroscopy of lignocellulosic materials treated with maleated polypropylenes. Journal of Wood Chemistry and Technology 18(1):1-26.

86. Kelley, S.S., T.G. Rials, and W.G. Glasser. 1987. Relaxation behavior of the amorphous components of wood. Chapman and Hall Ltd. 617-624.

87. Kinloch, A.J. 1987. Adhesion and Adhesives: Science and Technology. Chapman \& Hall, London, UK, $441 \mathrm{p}$.

88. Koch, P. 1964. Techniques for Drying Thick Southern Pine Veneer. Forest Product Journal 14(9):382-386.

89. Koubaa, A., B. Riedel, and Z. Koran. 1996. Surface analysis of press dried-CTMP paper samples by electron spectroscopy for chemical analysis. Journal of Applied Polymer Science 61:545-552.

90. Kozlik, C.J. 1974. Effect of temperature, time, and drying medium on the strength and gluability of Douglas-fir and southern pine veneer. Forest Product Journal 24(2):46-53.

91. Li, K, and D.W. Reeve. 2000. The origins of kraft pulp fibre surface lignin. International Pulp Bleaching Conference Proceedings, PAPTAC, Halifax, Nova Scotia, 197-202.

92. Liptakova, E., J. Kudela, Z. Bastl, and I. Spirovova. 1995. Influence of mechanical surface treatment of wood on the wetting process. Holzforschung 49(4):369-375.

93. Liu, F.P. and T.G. Rials. 1998. Relationship of wood surface energy to surface composition. Langmuir 14:536-541.

94. Löscher, F., T. Ruckstuhl, T. Jaworek, G. Wegner, and S. Seeger. 1998. Immobilization of biomolecules on Langmuir-Blodgett films of regenerative cellulose derivatives. Langmuir 14(10):2786-2789.

95. Lu, J.Z., Q. Wu, and H.S. McNabb, Jr. 2000. Chemical coupling in wood fiber and polymer composites: A review of coupling agents and treatments. Wood and Fiber Science 32(1):88-104. 
96. Maldas, D.C. and P.D. Kamdem. 1998. Surface tension and wettability of CCA-treated red maple. Wood and Fiber Science 30(4):368-373.

97. Mann, P.S. 1995. Introductory Statistics. John Wiley\&Sons, Inc. New York, 380-430.

98. Manninen, A-M., P. Pasanen, and J.K. Holopainen. 2002. Comparing the VOC emissions between air-dried and heat-treated Scots pine wood. Atmospheric Environment (in press).

99. Mantanis, G.I. and R.A. Young. 1997. Wetting of wood. Wood Science and Technology 31:339-353.

100. Marra, A. A. 1992. Technology of Wood Bonding: Principles in Practice. Van Nostrand Reinhold. New York, 454 p.

101. McGraw, G.W., R.W. Hemingway, L.L. Ingram, Jr., C.S. Canady, and W.B. McGraw. 1999. Thermal degradation of terpenes: Camphene, $\Delta^{3}$-Carene, Limonene, and $\alpha$ Terpinene. Environmental Science \& Technology 33(22):4029-4033.

102. Mjöberg, P.J. 1981. Chemical surface analysis of wood fibers by means of ESCA. Cellulose Chemistry and Technology 15:481-486.

103. Mohseni, M. and D.G. Allen. 2000. Biofiltration of mixtures of hydrophilic and hydrophobic volatile organic compounds. Chemical Engineering Science 55:1545-1558.

104. Nguyen, T. and W.E. Johns. 1978. Polar and dispersion force contributions to the total surface free energy of wood. Wood Science and Technology 12:63-74.

105. Nguyen, T. and W.E. Johns. 1979. The effect of aging and extraction on the surface free energy of Douglas-fir and redwood. Wood Science and Technology 12:29-40.

106. Northcott, P.L. 1957. The Effect of Dryer Temperatures Upon the Gluing Properties of Douglas-fir Veneer. Forest Product Journal 7(1):10-16.

107. Northcott, P.L., H.G.M. Colbeck, W.V. Hancock, and K.C. Shen. 1959. Casehardening in plywood. Forest Products Journal 10(12):442-451.

108. Northey, R. 2002. Wood Extractives. http://courses.washington.edu/pse409/.

109. Ostmeyer, J.G., T.J. Elder, D.M. Littrell, B.J. Tatarchuk, and J.E. Winandy. 1988. Spectroscopic analysis of southern pine treated with chromated copper arsenate. Journal of Wood Chemistry and Technology 8(3):413-439. 
110. Pettersen, R.C. 1984. The Chemical Composition of Wood. Chapter 2 in The Chemistry of Solid Wood. Editor R.M. Rowell, ACS Washington, D.C., 57-126.

111. Pizzi, A. 1983. Wood Adhesives Chemistry and Technology. Marcel Dekker, Inc. New York, 364 p.

112. Pizzi, A. 1994. Advanced Wood Adhesives Technology. Marcel Dekker, Inc. New York, 289 p.

113. Podgorski, L., B. Chevet, L. Onic, and A. Merlin. 2000. Modification of wood wettability by plasma and corona treatments. International Journal of Adhesion and Adhesives 20:103-111.

114. Reeve, D.W. and Z. Tan. 1998. The study of carbon-chlorine bonds in bleached bulp with X-ray photoelectron spectroscopy. Journal of Wood Chemistry and Technology 18 (4):417-426.

115. Rowe, J.W. 1989. Natural Products of Woody Plants I and II. Springer-Verlag, Berlin, $1243 \mathrm{p}$.

116. Rowell, R.M. 1984. The Chemistry of Solid Wood. American Chemical Society, Washington, D.C., 614 p.

117. Rowell, R.M. 1995. Chemical modification of wood for improved adhesion in composites. Pages 56-60 in: Christiansen, A.W. and A.H. Conner, editors. Wood Adhesives 1995. Proceedings No. 7296, Forest Product Society, Madison, WI.

118. Scheikl, M. and M. Dunky. 1998. Measurement of dynamic and static contact angles on wood for the determination of its surface tension and the penetration of liquids into the wood surface. Holzforschung 52(1):89-94.

119. Schmidt, R.G. 1998. Aspects of Wood Adhesion: Applications of Wood ${ }^{13}$ C CP/MAS NMR and Fracture Testing (Dissertation). Blacksburg (VA): Virginia Polytechnic Institute and State University. 140 p.

120. Schönberg, C., T. Oksanen, A. Suurnäkki, H. Kettunen, and J. Buchert. 2001. The importance of xylan for the strength properties of spruce kraft pulp fibers. Holzforschung $55(6): 639-644$. 
121. Schrader, E.M. and G.I. Loeb. 1992. Modern Approaches to Wettability. Theory and Applications. Plenum Press. New York and London, 451 p.

122. Sellers, T. 1977. A Plywood Review and its Chemical Implications, Wood Technology: Chemical Aspects. In: Goldstein, I.S., editor. Symposium Series No. 43, American Chemical Society, Washington, D.C.

123. Sellers, T. 1985. Plywood and Adhesive Technology. Marcel Dekker, Inc., New York, $661 \mathrm{p}$.

124. Sernek, M., J. Resnik and F.A. Kamke. 1999. Penetration of liquid urea-formaldehyde adhesive into beech wood. Wood and Fiber Science 31(1):41-48.

125. Shi, S.Q. and D.J. Gardner. 2001. Dynamic adhesive wettability of wood. Wood and Fiber Science 33(1):58-68.

126. Shupe, T.F., C.Y. Hse, and W.H. Wang. 2001. An investigation of selected factors that influence hardwood wettability. Holzforschung 55(5):541-548.

127. Siau, J.F. 1995. Wood: Influence of Moisture on Physical Properties. Virginia Polytechnic Institute and State University. 227 p.

128. Sjöström, E. 1993. Wood Chemistry: Fundamentals and Applications. Academic Press, San Diego, 293 p.

129. Stanley, R.G. 1969. Extractives of wood, bark, and needles of the southern pines. A review. Forest Products Journal 19(11):50-56.

130. Stenius, P. and T. Vuorinen. 1999. Direct Characterization of Chemical Properties of Fibers. Pages 149-192 in: Sjöström, E. and R. Alén, editors. Analytical Methods in Wood Chemistry, Pulping, and Papermaking.

131. Su, W., Yan, H., Banerjee, S., Otwell, L. P., and Hittmeier, M. E. 1999. Field proven strategies for reducing volatile organic carbons from hardwood drying. Environmental Science \& Technology 33(7):1056-1059.

132. Subramanian, R.V. 1984. Chemistry of adhesion. Pages 323-348 in Rowell, R.M., editor. The Chemistry of Solid Wood. American Chemical Society, Washington, D.C.

133. Suchsland, O., and R.R. Stevens. 1968. Gluability of southern pine veneer dried at high temperatures. Forest Products Journal 18(1):38-42. 
134. Troughton, G. 2001. Veneer Drying Manual. Special publication SP-45. Forintek Canada Corp. 62 p.

135. Troughton, G.E., and S.Z. Chow. 1971. Migration of fatty acids to white spruce veneer surface during drying: Relevance to theories of inactivation. Wood Science 3(3):129-133.

136. Tsujii, K. 1998. Surface Activity. Principles, Phenomena, and Applications. Academic Press, San Diego 245 p.

137. USDA 1979. Extractives in Eastern Hardwoods-A Review. General technical report FPL 18. Forest Products Laboratory, Forest Service, U.S. Department of Agriculture. Madison, WI, $67 \mathrm{p}$.

138. USDA 1999. Wood Handbook. Wood as an Engineering Material/United States Department of Agriculture Forest Service, Forest Products Laboratory. Madison, WI, USDA, Forest Service, 463 p.

139. Vick, C.B. 1995. Hydroxymethylated resorcinol coupling agent for enhanced adhesion of epoxy and other thermosetting adhesives to wood. Pages 47-55 in Christiansen, A.W. and A.H. Conner, editors. Wood Adhesives 1995. Proceedings No. 7296, Forest Product Society, Madison, WI.

140. Vick, C.B., K.H. Richter, and B.H. River. 1996. Hydroxymethylated resorcinol coupling agent and method for bonding wood. United States Patent, 5,543,487. US Patent \& Trademark Office, $19 \mathrm{p}$.

141. Vick, C.B., A.W. Christiansen, and E.A. Okkonen. 1998. Reactivity of hydroxymethylated resorcinol coupling agent as it affects durability of epoxy bonds to Douglasfir. Wood and Fiber Science 30(3):312-322.

142. Vick, C.B. and E.A. Okkonen. 2000. Durability of one-part polyurethane bonds to wood improved by HMR coupling agent. Forest Products Journal 50(10):69-75.

143. Wålinder, M. and I. Johansson. 2001. Measurement of wood wettability by the Wilhelmy method. Part 1. Contamination of probe liquids by extractives. Holzforschung 55(1):2132. 
144. Walters, E.O. 1973. The effect of green veneer water content, dryer schedules, and wettability on gluing results for southern pine veneer. Forest Product Journal 23(6):4653.

145. Wegman, R.F. 1989. Surface Preparation Techniques for Adhesive Bonding. Noyes Publications, U.S.A., 150 p.

146. Wellons, J. D. 1977. Adhesion to wood substrates. Pages 150-168 in: Gould, R. F., editor. ACS Symposium Series, American Chemical Society.

147. Wellons, J.D. 1980. Wettability and gluability of Douglas-fir veneer. Forest Product Journal 30(7):53-55.

148. White, M.S., G. Ifju, and J.A. Johnson. 1974. The role of extractives in the hydrophobic behavior of loblolly pine rhytidome. Wood and Fiber Science 5(4):353-363.

149. White, M.S., G. Ifju, and J.A. Johnson. 1977. Method of measuring resin penetration into wood. Forest Products Journal 27(7):52-55.

150. White, R.H. 1987. Effect of lignin content and extractives on the higher heating values of wood. Wood and Fiber Science 19(4):446-452.

151. Widsten, P., J.E. Laine, P. Qvintus-Leino, and S. Tuominen. 2002. Effect of hightemperature defibration on the chemical structure of hardwood. Holzforschung 56(1):5659.

152. Winandy, J.E. and R.M. Rowell. 1984. The chemistry of wood strength. Pages 211-255 in: Rowell, R.M., editor. The Chemistry of Solid Wood. American Chemical Society, Washington, D.C.

153. Winfield, P.H., A.F. Harris, and A.R. Hutchinson. 2001. The use of flame ionization technology to improve the wettability and adhesion properties of wood. International Journal of Adhesion \& Adhesives 21:107-114.

154. Wu, J. and M.R. Milota. 1999. Effect of temperature and humidity on total hydrocarbon emissions from Douglas-fir lumber. Forest Products Journal 49(6):52-60.

155. Yoshimoto, T. 1989. Effect of extractives on the utilization of wood. Pages 920-931 in Natural Products of Woody Plants II. Editor Rowe, J.W. Springer-Verlag, Berlin. 
156. Young, R.A., R.M. Rammon, S.S. Kelley, and R.H. Gillespie. 1982. Bond formation by wood surface reactions: Part I - Surface analysis by ESCA. Wood Science 14(3):110-119.

157. Zavarin, E. 1984. Activation of wood surface and nonconventional bonding. Pages 349400 in: Rowell, R.M., editor. The Chemistry of Solid Wood. American Chemical Society, Washington, D.C.

158. Zavarin, E. and L. Cool. 1991. Extraneous Materials from Wood. Pages 321-407 in: Lewin, M. and I.S. Goldstein, editors. Wood Structure and Composition. Marcel Dekker, Inc. New York.

159. Zhang, H.J., D.J. Gardner, J.Z. Wang, and Q. Shi. 1997. Surface tension, adhesive wettability, and bondability of artificially weathered CCA-treated southern pine. Forest Products Journal 47(10):69-72.

160. Zheng, J. and C.E. Frazier. 2002. Personal communication.

161. Zisman, W. A. 1964. Relation of the Equilibrium Contact Angle to Liquid and Solid Constitution. In Contact Angle, Wettability and Adhesion. American Chemical Society, Washington, D.C., 389 p. 


\section{Appendix}

Appendix A. X-ray photoelectron spectroscopy results for wood surfaces.

\begin{tabular}{|c|c|c|c|c|c|c|c|c|c|c|c|}
\hline \multirow{2}{*}{$\begin{array}{c}\text { Wood } \\
\text { Species }\end{array}$} & \multirow{2}{*}{$\begin{array}{l}\text { Sample } \\
\text { Name }\end{array}$} & \multirow{2}{*}{$\begin{array}{l}\text { Number of } \\
\text { Replication }\end{array}$} & \multicolumn{9}{|c|}{ Atomic Percent $(\%)$} \\
\hline & & & C1s & O1s & N1s & C1 & $\mathrm{C} 2$ & $\mathrm{C3}$ & $\mathbf{0 1}$ & $\overline{02}$ & $\overline{03}$ \\
\hline \multirow[t]{20}{*}{ Yellow-poplar } & YP50/1 & 1 & 75.4 & 24.0 & 0.6 & 59.2 & 34.0 & 6.8 & 12.2 & 79.3 & 8.6 \\
\hline & YP50/2 & 2 & 74.7 & 24.8 & 0.5 & 58.8 & 34.7 & 6.6 & 12.2 & 78.9 & 8.9 \\
\hline & YP50/(3) & 3 & 75.7 & 23.8 & 0.4 & 64.1 & 28.4 & 7.6 & 10.9 & 76.8 & 12.3 \\
\hline & & AVERAGE & 75.3 & 24.2 & 0.5 & 60.7 & 32.4 & 7.0 & 11.8 & 78.3 & 9.9 \\
\hline & YP100/(1) & 1 & 73.3 & 26.0 & 0.8 & 55.2 & 35.1 & 9.8 & 9.6 & 77.8 & 12.6 \\
\hline & YP100/2 & 2 & 75.8 & 23.6 & 0.6 & 61.0 & 31.0 & 8.0 & 10.5 & 81.4 & 8.1 \\
\hline & YP100/3 & 3 & 78.7 & 20.9 & 0.4 & 64.9 & 29.0 & 6.1 & 11.2 & 79.3 & 9.5 \\
\hline & & AVERAGE & 75.9 & 23.5 & 0.6 & 60.4 & 31.7 & 8.0 & 10.4 & 79.5 & 10.1 \\
\hline & YP150/1 & 1 & 77.3 & 22.0 & 0.6 & 66.4 & 25.6 & 8.1 & 16.4 & 73.9 & 9.7 \\
\hline & YP150/2 & 2 & 72.8 & 26.3 & 0.8 & 54.4 & 35.9 & 9.8 & 19.1 & 70.3 & 10.5 \\
\hline & YP150/3 & 3 & 74.4 & 25.1 & 0.6 & 57.4 & 34.6 & 8.0 & 16.8 & 75.3 & 7.9 \\
\hline & & AVERAGE & 74.8 & 24.5 & 0.7 & 59.4 & 32.0 & 8.6 & 17.4 & 73.2 & 9.4 \\
\hline & YP175/1 & 1 & 76.1 & 23.0 & 0.8 & 58.0 & 34.1 & 7.9 & 30.8 & 61.7 & 7.5 \\
\hline & YP175/2 & 2 & 81.1 & 18.0 & 0.9 & 67.5 & 25.8 & 6.7 & 23.7 & 66.9 & 9.4 \\
\hline & YP175/3 & 3 & 78.2 & 21.1 & 0.7 & 66.3 & 26.1 & 7.6 & 19.9 & 70.7 & 9.4 \\
\hline & & AVERAGE & 78.5 & 20.7 & 0.8 & 63.9 & 28.7 & 7.4 & 24.8 & 66.4 & 8.8 \\
\hline & YP200/1 & 1 & 81.4 & 18.2 & 0.4 & 73.8 & 20.8 & 5.5 & 40.0 & 52.0 & 8.0 \\
\hline & YP200/2 & 2 & 77.4 & 22.2 & 0.4 & 63.2 & 30.6 & 6.3 & 23.9 & 67.3 & 8.8 \\
\hline & YP200/3 & 3 & 85.2 & 14.6 & 0.2 & 80.2 & 15.3 & 4.5 & 29.8 & 59.3 & 10.9 \\
\hline & & AVERAGE & 81.3 & 18.3 & 0.3 & 72.4 & 22.2 & 5.4 & 31.3 & 59.5 & 9.2 \\
\hline \multirow[t]{20}{*}{ Southern Pine } & SP50/1 & 1 & 79.7 & 20.0 & 0.3 & 74.2 & 18.0 & 7.8 & 13.9 & 60.9 & 25.2 \\
\hline & SP50/(2) & 2 & 81.0 & 18.0 & 1.0 & 74.5 & 16.5 & 9.0 & 16.5 & 62.1 & 21.5 \\
\hline & SP50/3 & 3 & 79.9 & 19.5 & 0.6 & 73.1 & 18.9 & 8.0 & 13.9 & 61.4 & 24.8 \\
\hline & & AVERAGE & 80.5 & 18.8 & 0.8 & 73.8 & 17.7 & 8.5 & 15.2 & 61.7 & 23.1 \\
\hline & SP100/1 & 1 & 78.7 & 20.4 & 0.9 & 71.0 & 20.9 & 8.1 & 15.5 & 63.0 & 21.4 \\
\hline & SP100/2 & 2 & 81.2 & 18.2 & 0.7 & 71.8 & 20.3 & 7.9 & 13.1 & 65.3 & 21.7 \\
\hline & SP100/(3) & 3 & 80.8 & 18.7 & 0.6 & 76.0 & 16.1 & 8.0 & 15.2 & 64.3 & 20.5 \\
\hline & & AVERAGE & 80.2 & 19.1 & 0.7 & 72.9 & 19.1 & 8.0 & 14.6 & 64.2 & 21.2 \\
\hline & SP150/(1) & 1 & 82.9 & 16.5 & 0.5 & 75.3 & 17.6 & 7.1 & 14.6 & 64.0 & 21.3 \\
\hline & SP150/2 & 2 & 83.9 & 15.7 & 0.4 & 78.9 & 15.5 & 5.6 & 13.9 & 62.1 & 24.0 \\
\hline & SP150/3 & 3 & 85.7 & 13.9 & 0.5 & 80.0 & 13.9 & 6.2 & 17.0 & 59.6 & 23.4 \\
\hline & & AVERAGE & 84.2 & 15.4 & 0.5 & 78.1 & 15.7 & 6.3 & 15.2 & 61.9 & 22.9 \\
\hline & SP175/(1) & 1 & 86.8 & 13.2 & 0.0 & 85.8 & 10.1 & 4.2 & 15.0 & 59.1 & 25.9 \\
\hline & SP175/2 & 2 & 82.7 & 16.8 & 0.5 & 76.1 & 16.4 & 7.5 & 15.2 & 61.2 & 23.6 \\
\hline & SP175/3 & 3 & 82.5 & 17.0 & 0.6 & 74.6 & 18.1 & 7.4 & 10.3 & 67.2 & 22.5 \\
\hline & & AVERAGE & 84.0 & 15.6 & 0.4 & 78.8 & 14.8 & 6.3 & 13.5 & 62.5 & 24.0 \\
\hline & SP200/1) & 1 & 86.2 & 13.4 & 0.5 & 80.6 & 13.3 & 6.1 & 19.5 & 65.1 & 15.4 \\
\hline & SP200/2 & 2 & 84.6 & 15.0 & 0.4 & 76.2 & 17.1 & 6.7 & 18.9 & 57.6 & 23.5 \\
\hline & SP200/3 & 3 & 85.0 & 14.5 & 0.6 & 76.8 & 16.9 & 6.3 & 18.7 & 58.0 & 23.3 \\
\hline & & AVERAGE & 85.2 & 14.3 & 0.5 & 77.9 & 15.8 & 6.4 & 19.0 & 60.2 & 20.7 \\
\hline
\end{tabular}


Appendix B. Water contact angles of yellow-poplar.

\begin{tabular}{|c|c|c|c|c|c|c|c|c|c|}
\hline \multirow{2}{*}{$\begin{array}{l}\text { Wood } \\
\text { Species }\end{array}$} & \multirow{2}{*}{$\begin{array}{c}\text { Max. Surface. T. } \\
\left({ }^{\circ} \mathrm{C}\right)\end{array}$} & \multirow{2}{*}{$\begin{array}{l}\text { Number of } \\
\text { Replication }\end{array}$} & \multicolumn{7}{|c|}{ Contact Angle $\left({ }^{\circ}\right)$} \\
\hline & & & $0 \mathrm{~s}$. & $10 \mathrm{~s}$. & $20 \mathrm{~s}$. & $30 \mathrm{~s}$. & $40 \mathrm{~s}$. & $50 \mathrm{~s}$. & $60 \mathrm{~s}$. \\
\hline \multirow[t]{10}{*}{ Yellow-Poplar } & \multirow[t]{10}{*}{51} & 1 & 57.4 & 38.2 & 35.7 & 29.4 & 25.4 & 16.8 & 14.1 \\
\hline & & 2 & 54.9 & 23.3 & 15.3 & 5.2 & 0.0 & 0.0 & 0.0 \\
\hline & & 3 & 58.8 & 33.5 & 29.6 & 27.7 & 22.6 & 17.4 & 15.5 \\
\hline & & 4 & 50.4 & 33.2 & 17.9 & 5.3 & 0.0 & 0.0 & 0.0 \\
\hline & & 5 & 64.5 & 60.0 & 56.3 & 51.4 & 49.2 & 46.6 & 39.6 \\
\hline & & 6 & 71.0 & 58.1 & 54.9 & 50.3 & 45.2 & 42.8 & 39.9 \\
\hline & & & 6 & 6 & 6 & 6 & 6 & 6 & 6 \\
\hline & & AVERAGE & 59.5 & 41.1 & 35.0 & 28.2 & 23.7 & 20.6 & 18.2 \\
\hline & & STDEV & 7.3 & 14.8 & 17.7 & 20.4 & 21.2 & 20.2 & 18.0 \\
\hline & & $\operatorname{COV}(\%)$ & 12.3 & 36.0 & 50.6 & 72.4 & 89.2 & 98.1 & 98.9 \\
\hline \multirow[t]{10}{*}{ Yellow-Poplar } & \multirow[t]{10}{*}{104} & 1 & 61.2 & 47.6 & 45.9 & 39.2 & 36.2 & 35.3 & 34.4 \\
\hline & & 2 & 62.2 & 51.2 & 48.0 & 39.3 & 37.6 & 36.9 & 35.2 \\
\hline & & 3 & 59.4 & 37.0 & 32.5 & 30.2 & 25.3 & 21.6 & 20.0 \\
\hline & & 4 & 74.9 & 54.4 & 51.2 & 50.2 & 48.8 & 46.2 & 44.7 \\
\hline & & 5 & 59.3 & 47.2 & 44.2 & 40.0 & 35.0 & 32.7 & 30.5 \\
\hline & & 6 & 53.3 & 44.4 & 43.4 & 42.3 & 41.1 & 40.6 & 39.8 \\
\hline & & & 6 & 6 & 6 & 6 & 6 & 6 & 6 \\
\hline & & AVERAGE & 61.7 & 47.0 & 44.2 & 40.2 & 37.3 & 35.6 & 34.1 \\
\hline & & STDEV & 7.2 & 6.0 & 6.4 & 6.4 & 7.7 & 8.3 & 8.4 \\
\hline & & $\operatorname{COV}(\%)$ & 11.6 & 12.7 & 14.5 & 16.0 & 20.6 & 23.3 & 24.8 \\
\hline \multirow[t]{10}{*}{ Yellow-Poplar } & \multirow[t]{10}{*}{156} & 1 & 71.5 & 59.3 & 56.4 & 46.0 & 41.5 & 40.2 & 39.0 \\
\hline & & 2 & 71.6 & 61.5 & 58.3 & 56.2 & 53.5 & 50.1 & 46.8 \\
\hline & & 3 & 71.3 & 55.9 & 53.0 & 49.4 & 48.4 & 45.9 & 41.2 \\
\hline & & 4 & 72.0 & 51.2 & 49.1 & 45.4 & 42.1 & 39.6 & 39.6 \\
\hline & & 5 & 70.5 & 58.1 & 54.8 & 46.1 & 41.8 & 41.2 & 38.1 \\
\hline & & & 71.4 & 56.7 & 51.2 & 50.0 & 49.3 & 48.7 & 48.4 \\
\hline & & & 6 & 6 & 6 & 6 & 6 & 6 & \\
\hline & & AVERAGE & 71.4 & 57.1 & 53.8 & 48.8 & 46.1 & 44.3 & 42.2 \\
\hline & & STDEV & 0.5 & 3.5 & 3.4 & 4.1 & 5.0 & 4.6 & 4.3 \\
\hline & & $\operatorname{COV}(\%)$ & 0.7 & 6.1 & 6.3 & 8.4 & 10.9 & 10.3 & 10.3 \\
\hline \multirow[t]{10}{*}{ Yellow-Poplar } & \multirow[t]{10}{*}{172} & 1 & 73.8 & 61.1 & 55.6 & 53.4 & 50.3 & 48.4 & 47.4 \\
\hline & & 2 & 77.4 & 65.8 & 56.1 & 47.7 & 46.9 & 45.9 & 45.0 \\
\hline & & 3 & 68.7 & 50.6 & 42.3 & 37.0 & 35.7 & 35.7 & 35.7 \\
\hline & & 4 & 64.3 & 42.0 & 39.4 & 39.4 & 36.9 & 36.3 & 35.5 \\
\hline & & 5 & 75.1 & 58.4 & 56.1 & 50.4 & 41.5 & 38.2 & 38.2 \\
\hline & & 6 & 74.7 & 51.3 & 43.4 & 42.2 & 36.4 & 35.2 & 35.2 \\
\hline & & & 6 & 6 & 6 & 6 & 6 & 6 & \\
\hline & & AVERAGE & 72.3 & 54.8 & 48.8 & 45.0 & 41.3 & 40.0 & 39.5 \\
\hline & & STDEV & 4.9 & 8.6 & 7.9 & 6.5 & 6.1 & 5.7 & 5.3 \\
\hline & & $\operatorname{COV}(\%)$ & 6.7 & 15.6 & 16.2 & 14.4 & 14.9 & 14.3 & 13.5 \\
\hline \multirow[t]{10}{*}{ Yellow-Poplar } & \multirow[t]{10}{*}{187} & 1 & 75.7 & 53.3 & 50.7 & 49.6 & 49.0 & 48.5 & 47.5 \\
\hline & & 2 & 72.6 & 55.0 & 53.4 & 52.9 & 52.1 & 51.9 & 51.7 \\
\hline & & 3 & 70.9 & 55.5 & 52.5 & 48.5 & 46.8 & 45.7 & 45.0 \\
\hline & & 4 & 79.8 & 57.2 & 51.2 & 45.1 & 44.9 & 44.6 & 44.4 \\
\hline & & 5 & 92.5 & 90.1 & 75.3 & 74.9 & 73.2 & 69.6 & 69.3 \\
\hline & & 6 & 73.5 & 63.8 & 60.7 & 58.1 & 58.1 & 58.1 & 58.1 \\
\hline & & & 6 & 6 & 6 & 6 & 6 & 6 & \\
\hline & & AVERAGE & $\mathbf{7 7 . 5}$ & 62.5 & $\mathbf{5 7 . 3}$ & 54.8 & 54.0 & 53.1 & 52.7 \\
\hline & & STDEV & 8.0 & 14.0 & 9.5 & 10.8 & 10.5 & 9.5 & 9.6 \\
\hline & & $\operatorname{COV}(\%)$ & 10.3 & 22.4 & 16.6 & 19.7 & 19.4 & 17.8 & 18.2 \\
\hline
\end{tabular}


Appendix C. Water contact angles of southern pine.

\begin{tabular}{|c|c|c|c|c|c|c|c|c|c|}
\hline \multirow{2}{*}{$\begin{array}{c}\text { Wood } \\
\text { Species }\end{array}$} & \multirow{2}{*}{$\begin{array}{c}\text { Max. Surface T. } \\
\left({ }^{\circ} \mathrm{C}\right)\end{array}$} & \multirow{2}{*}{$\begin{array}{l}\text { Number of } \\
\text { Replication }\end{array}$} & \multicolumn{7}{|c|}{ Contact Angle $\left({ }^{\circ}\right)$} \\
\hline & & & $0 \mathrm{~s}$. & $10 \mathrm{~s}$. & $20 \mathrm{~s}$. & $30 \mathrm{~s}$. & $40 \mathrm{~s}$. & $50 \mathrm{~s}$. & $60 \mathrm{~s}$. \\
\hline \multirow[t]{10}{*}{ Southern Pine } & \multirow[t]{10}{*}{51} & 1 & 81.9 & 60.1 & 56.1 & 51.2 & 47.8 & 40.4 & 39.4 \\
\hline & & 2 & 76.2 & 62.6 & 48.8 & 43.5 & 37.2 & 32.2 & 31.0 \\
\hline & & 3 & 88.5 & 73.2 & 60.6 & 55.2 & 50.7 & 47.8 & 47.0 \\
\hline & & 4 & 86.2 & 72.7 & 62.8 & 52.9 & 49.0 & 44.6 & 38.2 \\
\hline & & 5 & 82.3 & 70.5 & 58.4 & 55.5 & 50.5 & 49.3 & 44.7 \\
\hline & & 6 & 86.7 & 54.7 & 44.5 & 42.5 & 41.0 & 39.5 & 37.2 \\
\hline & & $\mathrm{n}$ & 6 & 6 & 6 & 6 & 6 & 6 & 0 \\
\hline & & AVERAGE & 83.6 & 65.6 & 55.2 & 50.1 & 46.0 & 42.3 & 39.6 \\
\hline & & STDEV & 4.5 & 7.6 & 7.1 & 5.8 & 5.6 & 6.3 & 5.7 \\
\hline & & $\operatorname{COV}(\%)$ & 5.3 & 11.6 & 12.9 & 11.5 & 12.2 & 14.9 & 14.4 \\
\hline \multirow[t]{10}{*}{ Southern Pine } & \multirow[t]{10}{*}{104} & 1 & 89.1 & 76.9 & 68.2 & 57.6 & 53.3 & 50.3 & 47.9 \\
\hline & & 2 & 95.4 & 87.6 & 83.9 & 71.1 & 60.2 & 55.1 & 51.9 \\
\hline & & 3 & 92.7 & 85.1 & 82.2 & 78.3 & 75.3 & 70.7 & 69.7 \\
\hline & & 4 & 95.9 & 92.7 & 90.0 & 88.2 & 87.3 & 84.7 & 80.2 \\
\hline & & 5 & 85.5 & 84.2 & 72.1 & 69.4 & 66.3 & 65.5 & 60.8 \\
\hline & & 6 & 83.1 & 66.9 & 64.6 & 63.2 & 62.2 & 61.1 & 61.1 \\
\hline & & & 6 & 6 & 6 & 6 & 6 & 6 & \\
\hline & & AVERAGE & 90.3 & 82.2 & 76.8 & 71.3 & 67.4 & 64.6 & 61.9 \\
\hline & & STDEV & 5.3 & 9.1 & 10.0 & 10.9 & 12.1 & 12.2 & 11.8 \\
\hline & & $\operatorname{COV}(\%)$ & 5.9 & 11.0 & 13.0 & 15.3 & 18.0 & 19.0 & 19.0 \\
\hline \multirow[t]{10}{*}{ Southern Pine } & \multirow[t]{10}{*}{156} & 1 & 88.8 & 87.9 & 86.9 & 84.0 & 78.3 & 77.5 & 77.5 \\
\hline & & 2 & 89.6 & 71.2 & 68.3 & 64.8 & 62.0 & 60.9 & 60.1 \\
\hline & & 3 & 90.1 & 86.1 & 81.3 & 78.0 & 72.4 & 72.0 & 71.7 \\
\hline & & 4 & 100.2 & 89.1 & 82.3 & 76.9 & 72.1 & 70.5 & 68.8 \\
\hline & & 5 & 94.2 & 93.4 & 92.7 & 90.8 & 90.5 & 90.1 & 90.1 \\
\hline & & 6 & 123.8 & 116.8 & 111.1 & 109.4 & 107.8 & 106.6 & 105.4 \\
\hline & & & 6 & 6 & 6 & 6 & 6 & 6 & \\
\hline & & AVERAGE & 97.8 & 90.7 & 87.1 & 84.0 & 80.5 & 79.6 & 78.9 \\
\hline & & STDEV & 13.4 & 14.9 & 14.2 & 15.2 & 16.3 & 16.3 & 16.4 \\
\hline & & $\operatorname{COV}(\%)$ & 13.7 & 16.4 & 16.4 & 18.0 & 20.2 & 20.5 & 20.7 \\
\hline \multirow[t]{10}{*}{ Southern Pine } & \multirow[t]{10}{*}{172} & 1 & 104.5 & 99.1 & 98.8 & 98.0 & 97.0 & 97.0 & 96.1 \\
\hline & & 2 & 98.9 & 97.3 & 94.0 & 90.6 & 90.6 & 90.1 & 88.8 \\
\hline & & 3 & 93.7 & 74.7 & 66.3 & 60.3 & 60.3 & 58.0 & 58.0 \\
\hline & & 4 & 97.7 & 76.8 & 66.1 & 61.1 & 60.2 & 60.2 & 57.4 \\
\hline & & 5 & 92.2 & 87.2 & 85.1 & 83.6 & 82.2 & 81.3 & 80.4 \\
\hline & & 6 & 96.8 & 94.0 & 92.9 & 91.2 & 90.8 & 88.9 & 87.9 \\
\hline & & $\mathrm{n}$ & 6 & 6 & 6 & 6 & 6 & 6 & 6 \\
\hline & & AVERAGE & 97.3 & 88.2 & 83.8 & 80.8 & 80.2 & 79.3 & 78.1 \\
\hline & & STDEV & 4.3 & 10.5 & 14.4 & 16.2 & 16.2 & 16.4 & 16.6 \\
\hline & & $\operatorname{COV}(\%)$ & 4.5 & 11.9 & 17.1 & 20.1 & 20.2 & 20.7 & 21.2 \\
\hline \multirow[t]{10}{*}{ Southern Pine } & \multirow[t]{10}{*}{187} & 1 & 112.1 & 108.1 & 105.1 & 105.1 & 105.1 & 105.0 & $\overline{104.2}$ \\
\hline & & 2 & 95.2 & 86.4 & 85.0 & 84.2 & 84.2 & 83.9 & 83.9 \\
\hline & & 3 & 93.2 & 90.4 & 88.0 & 86.6 & 85.0 & 83.8 & 82.5 \\
\hline & & 4 & 99.0 & 86.2 & 84.6 & 82.0 & 81.7 & 81.5 & 81.5 \\
\hline & & 5 & 97.0 & 93.0 & 90.3 & 90.3 & 89.7 & 89.2 & 88.4 \\
\hline & & 6 & 97.3 & 91.4 & 91.1 & 90.2 & 89.0 & 89.0 & 89.0 \\
\hline & & & 6 & 6 & 6 & 6 & 6 & 6 & \\
\hline & & AVERAGE & 98.9 & 92.6 & 90.7 & 89.7 & 89.1 & 88.7 & 88.2 \\
\hline & & STDEV & 6.7 & 8.1 & 7.5 & 8.2 & 8.4 & 8.5 & 8.4 \\
\hline & & $\operatorname{COV}(\%)$ & 6.8 & 8.7 & 8.3 & 9.2 & 9.4 & 9.6 & 9.5 \\
\hline
\end{tabular}


Appendix D. Strain energy release rate results for yellow-poplar specimens bonded with polyvinyl-acetate (PVA) and phenol-formaldehyde $(P F)$ adhesives.

\begin{tabular}{|c|c|c|c|c|c|c|}
\hline \multicolumn{3}{|c|}{ MAX = Maximum, ARR = Arrested } & \multicolumn{4}{|c|}{ Strain Energy Release Rate $\left(\mathrm{J} / \mathrm{m}^{2}\right)$} \\
\hline \multirow{2}{*}{$\begin{array}{l}\text { Wood } \\
\text { Species }\end{array}$} & \multirow{2}{*}{$\begin{array}{c}\text { Max. Surface. T. } \\
\left({ }^{\circ} \mathrm{C}\right)\end{array}$} & \multirow{2}{*}{$\begin{array}{l}\text { Number of } \\
\text { Replication }\end{array}$} & \multicolumn{2}{|c|}{ PVA } & \multicolumn{2}{|c|}{$\overline{\mathbf{P F}}$} \\
\hline & & & MAX & ARR & MAX & ARR \\
\hline \multirow[t]{10}{*}{ Yellow-Poplar } & \multirow[t]{10}{*}{51} & 1 & 330.5 & 320.3 & 445.7 & 393.9 \\
\hline & & 2 & 274.1 & 265.4 & 449.4 & 393.5 \\
\hline & & 3 & 337.4 & 286.2 & 253.6 & 222.7 \\
\hline & & 4 & 382.4 & 355.8 & 329.4 & 264.5 \\
\hline & & 5 & 293.2 & 245.3 & 343.3 & 314.2 \\
\hline & & 6 & 274.5 & 240.8 & 390.1 & 342.9 \\
\hline & & & 6 & 6 & 6 & \\
\hline & & AVERAGE & 315.4 & 285.6 & 368.6 & 321.9 \\
\hline & & STDEV & 42.6 & 45.1 & 75.3 & 69.2 \\
\hline & & $\operatorname{COV}(\%)$ & 13.5 & 15.8 & 20.4 & 21.5 \\
\hline \multirow[t]{10}{*}{ Yellow-Poplar } & \multirow[t]{10}{*}{104} & 1 & 299.5 & 267.9 & 251.1 & 220.8 \\
\hline & & 2 & 343.1 & 297.8 & 335.7 & 269.8 \\
\hline & & 3 & 252.2 & 229.6 & 385.5 & 356.8 \\
\hline & & 4 & 254.6 & 224.8 & 229.2 & 209.7 \\
\hline & & & 393.9 & 349.3 & 326.4 & 232.8 \\
\hline & & & 340.2 & 312.8 & 411.2 & 370.7 \\
\hline & & & & & & \\
\hline & & AVERAGE & 313.9 & 280.4 & 323.2 & 276.8 \\
\hline & & STDEV & 55.7 & 48.9 & 71.9 & 70.5 \\
\hline & & $\operatorname{COV}(\%)$ & 17.7 & 17.4 & 22.2 & 25.5 \\
\hline \multirow[t]{10}{*}{ Yellow-Poplar } & \multirow[t]{10}{*}{156} & 1 & 355.8 & 339.5 & 296.2 & 268.5 \\
\hline & & 2 & 339.6 & 296.7 & 344.6 & 310.0 \\
\hline & & 3 & 468.6 & 412.2 & 319.6 & 285.8 \\
\hline & & 4 & 461.6 & 362.7 & 360.3 & 320.0 \\
\hline & & 5 & 351.2 & 313.9 & 312.9 & 268.4 \\
\hline & & 6 & 272.5 & 246.2 & 295.5 & 259.6 \\
\hline & & & 6 & 6 & 6 & 6 \\
\hline & & AVERAGE & 374.9 & 328.5 & 321.5 & 285.4 \\
\hline & & STDEV & 76.1 & 57.1 & 26.2 & 24.7 \\
\hline & & $\operatorname{COV}(\%)$ & 20.3 & 17.4 & 8.2 & 8.6 \\
\hline \multirow[t]{10}{*}{ Yellow-Poplar } & \multirow[t]{10}{*}{172} & 1 & 433.8 & 393.0 & 336.7 & 286.3 \\
\hline & & 2 & 262.3 & 207.6 & 362.2 & 312.1 \\
\hline & & 3 & 465.3 & 419.9 & 365.0 & 334.5 \\
\hline & & 4 & 413.5 & 313.7 & 196.2 & 162.5 \\
\hline & & 5 & 259.9 & 178.5 & 297.5 & 271.6 \\
\hline & & 6 & 357.6 & 319.1 & 247.1 & 217.0 \\
\hline & & & 6 & 6 & 6 & \\
\hline & & AVERAGE & 365.4 & 305.3 & 300.8 & 264.0 \\
\hline & & STDEV & 88.1 & 96.7 & 67.8 & 63.8 \\
\hline & & $\operatorname{COV}(\%)$ & 24.1 & 31.7 & 22.6 & 24.2 \\
\hline \multirow[t]{10}{*}{ Yellow-Poplar } & \multirow[t]{10}{*}{187} & 1 & 180.3 & 165.1 & 295.8 & 261.9 \\
\hline & & 2 & 371.1 & 343.8 & 236.4 & 209.9 \\
\hline & & 3 & 362.5 & 361.5 & 244.3 & 220.0 \\
\hline & & 4 & 368.3 & 338.1 & 369.8 & 323.0 \\
\hline & & 5 & 254.8 & 236.8 & 357.0 & 304.3 \\
\hline & & 6 & 311.0 & 282.0 & 415.9 & 380.2 \\
\hline & & & & 6 & 6 & \\
\hline & & AVERAGE & 308.0 & 287.9 & 319.9 & 283.2 \\
\hline & & STDEV & 77.1 & 75.9 & 72.6 & 65.2 \\
\hline & & $\operatorname{COV}(\%)$ & 25.0 & 26.4 & 22.7 & 23.0 \\
\hline
\end{tabular}


Appendix E. Strain energy release rate results for southern pine specimens bonded with polyvinyl-acetate (PVA) and phenol-formaldehyde $(P F)$ adhesives.

\begin{tabular}{|c|c|c|c|c|c|c|}
\hline \multicolumn{3}{|c|}{ MAX $=$ Maximum, ARR = Arrested } & \multicolumn{4}{|c|}{ Strain Energy Release Rate $\left(\mathrm{J} / \mathrm{m}^{2}\right)$} \\
\hline \multirow{2}{*}{$\begin{array}{l}\text { Wood } \\
\text { Species }\end{array}$} & \multirow{2}{*}{$\begin{array}{c}\text { Max. Surface. T. } \\
\left({ }^{\circ} \mathrm{C}\right)\end{array}$} & \multirow{2}{*}{$\begin{array}{l}\text { Number of } \\
\text { Replication }\end{array}$} & \multicolumn{2}{|c|}{ PVA } & \multicolumn{2}{|c|}{$\overline{\mathbf{P F}}$} \\
\hline & & & MAX & ARR & MAX & ARR \\
\hline \multirow[t]{10}{*}{ Southern Pine } & \multirow[t]{10}{*}{51} & 1 & 190.8 & 132.8 & 277.1 & 203.8 \\
\hline & & 2 & 189.3 & 166.6 & 205.2 & 163.6 \\
\hline & & 3 & 243.8 & 210.5 & 188.2 & 165.9 \\
\hline & & 4 & 188.7 & 157.1 & 189.8 & 168.0 \\
\hline & & 5 & 102.4 & 67.1 & 229.8 & 202.5 \\
\hline & & 6 & 103.6 & 77.3 & 286.8 & 249.9 \\
\hline & & & 6 & 6 & 6 & 6 \\
\hline & & AVERAGE & 169.8 & 135.2 & 229.5 & 192.3 \\
\hline & & STDEV & 55.8 & 55.0 & 43.4 & 33.6 \\
\hline & & $\operatorname{COV}(\%)$ & 32.9 & 40.7 & 18.9 & 17.5 \\
\hline \multirow[t]{10}{*}{ Southern Pine } & \multirow[t]{10}{*}{104} & 1 & 207.4 & 172.0 & 166.9 & 148.9 \\
\hline & & 2 & 200.6 & 130.2 & 177.6 & 149.8 \\
\hline & & & 187.9 & 143.2 & 318.4 & 253.7 \\
\hline & & & 157.5 & 117.6 & 264.9 & 229.7 \\
\hline & & & 133.5 & 91.8 & 118.2 & 96.8 \\
\hline & & & 139.4 & 94.8 & 252.7 & 198.3 \\
\hline & & & & 6 & & \\
\hline & & AVERAGE & 171.0 & 124.9 & 216.5 & 179.5 \\
\hline & & STDEV & 31.8 & 30.5 & 74.4 & 58.4 \\
\hline & & $\operatorname{COV}(\%)$ & 18.6 & 24.4 & 34.4 & 32.5 \\
\hline \multirow[t]{10}{*}{ Southern Pine } & \multirow[t]{10}{*}{156} & 1 & 72.6 & 58.8 & 148.3 & 130.0 \\
\hline & & 2 & 84.5 & 67.4 & 134.3 & 125.4 \\
\hline & & 3 & 204.8 & 156.4 & 177.7 & 156.0 \\
\hline & & 4 & 219.0 & 182.4 & 127.7 & 116.5 \\
\hline & & 5 & 187.5 & 154.7 & 31.1 & 22.0 \\
\hline & & 6 & 228.7 & 185.0 & 38.1 & 29.4 \\
\hline & & & 6 & 6 & 6 & 6 \\
\hline & & AVERAGE & 166.2 & 134.1 & 109.5 & 96.6 \\
\hline & & STDEV & 69.4 & 56.5 & 60.6 & 56.5 \\
\hline & & $\operatorname{COV}(\%)$ & 41.8 & 42.1 & 55.3 & 58.5 \\
\hline \multirow[t]{10}{*}{ Southern Pine } & \multirow[t]{10}{*}{172} & 1 & 165.4 & 140.5 & 49.2 & 29.6 \\
\hline & & 2 & 196.9 & 170.2 & 85.9 & 47.2 \\
\hline & & 3 & 106.8 & 83.7 & 188.9 & 148.2 \\
\hline & & 4 & 120.1 & 89.6 & 143.8 & 111.5 \\
\hline & & 5 & 238.2 & 178.6 & 236.0 & 212.3 \\
\hline & & 6 & 135.9 & 108.1 & 158.5 & 147.6 \\
\hline & & & & 6 & & \\
\hline & & AVERAGE & 160.6 & 128.4 & 143.7 & 116.0 \\
\hline & & STDEV & 50.0 & 40.8 & 67.9 & 68.6 \\
\hline & & $\operatorname{cov}(\%)$ & 31.2 & 31.8 & 47.2 & 59.1 \\
\hline \multirow[t]{10}{*}{ Southern Pine } & \multirow[t]{10}{*}{187} & 1 & 55.5 & 37.7 & 40.2 & 32.2 \\
\hline & & 2 & 60.7 & 45.1 & 103.3 & 91.1 \\
\hline & & 3 & 173.7 & 93.0 & 80.3 & 62.1 \\
\hline & & 4 & 99.0 & 78.1 & 84.8 & 70.1 \\
\hline & & 5 & 40.1 & 21.9 & 123.8 & 93.4 \\
\hline & & 6 & 68.8 & 55.4 & 21.9 & 15.5 \\
\hline & & & 6 & 6 & & \\
\hline & & AVERAGE & 83.0 & 55.2 & 75.7 & 60.7 \\
\hline & & STDEV & 48.5 & 26.3 & 38.3 & 31.4 \\
\hline & & $\operatorname{COV}(\%)$ & 58.5 & 47.7 & 50.6 & 51.7 \\
\hline
\end{tabular}


Appendix F. Effective and maximum penetration of phenol-formaldehyde adhesive.

\begin{tabular}{|c|c|c|c|c|c|}
\hline \multicolumn{2}{|c|}{$\mathrm{EP}=$ Effective, $\mathrm{MP}=$ Maximum } & \multicolumn{4}{|c|}{ PF Adhesive Penetration $(\mu \mathrm{m})$} \\
\hline \multirow{2}{*}{$\begin{array}{c}\text { Max. Surface. T. } \\
\left({ }^{\circ} \mathrm{C}\right)\end{array}$} & \multirow{2}{*}{$\begin{array}{l}\text { Number of } \\
\text { Replication }\end{array}$} & \multicolumn{2}{|c|}{ Yellow-Poplar } & \multicolumn{2}{|c|}{ Southern Pine } \\
\hline & & EP & MP & EP & MP \\
\hline \multirow[t]{16}{*}{51} & 1 & 15.5 & 250.6 & 7.9 & 83.0 \\
\hline & 2 & 27.1 & 330.1 & 33.8 & 386.8 \\
\hline & 3 & 9.5 & 320.2 & 12.8 & 67.6 \\
\hline & 4 & 26.2 & 222.1 & 22.5 & 344.6 \\
\hline & 5 & 28.1 & 279.2 & 15.9 & 141.6 \\
\hline & 6 & 22.9 & 270.3 & 16.8 & 193.3 \\
\hline & 7 & 19.5 & 206.0 & 10.6 & 86.3 \\
\hline & 8 & 25.1 & 287.1 & 11.7 & 106.8 \\
\hline & 9 & 9.4 & 435.1 & 15.9 & 114.6 \\
\hline & 10 & 26.7 & 239.7 & 10.6 & 104.7 \\
\hline & 11 & 16.2 & 546.4 & 11.0 & 74.6 \\
\hline & 12 & 12.6 & 416.7 & 16.6 & 185.7 \\
\hline & $\mathrm{n}$ & 12 & 12 & 12 & 12 \\
\hline & AVERAGE & 19.9 & 316.9 & 15.5 & 157.5 \\
\hline & STDEV & 7.0 & 101.3 & 7.0 & 105.6 \\
\hline & $\operatorname{COV}(\%)$ & 35.4 & 32.0 & 45.0 & 67.1 \\
\hline \multirow[t]{16}{*}{187} & 1 & 27.9 & 239.3 & 21.8 & 157.5 \\
\hline & 2 & 22.9 & 323.8 & 6.6 & 50.4 \\
\hline & 3 & 25.8 & 265.0 & 21.8 & 156.4 \\
\hline & 4 & 23.9 & 270.8 & 4.9 & 131.5 \\
\hline & 5 & 17.9 & 144.5 & 28.7 & 203.4 \\
\hline & 6 & 31.9 & 429.9 & 13.7 & 121.3 \\
\hline & 7 & 16.3 & 224.8 & 9.3 & 104.8 \\
\hline & 8 & 9.5 & 189.8 & 32.4 & 202.9 \\
\hline & 9 & 19.8 & 247.5 & 24.2 & 140.3 \\
\hline & 10 & 29.1 & 381.2 & 7.3 & 41.3 \\
\hline & 11 & 25.2 & 192.7 & 19.3 & 152.6 \\
\hline & 12 & 18.3 & 330.4 & 6.1 & 40.4 \\
\hline & & 12 & 12 & 12 & 12 \\
\hline & AVERAGE & 22.4 & 270.0 & 16.3 & 125.2 \\
\hline & STDEV & 6.3 & 83.2 & 9.6 & 56.8 \\
\hline & $\operatorname{COV}(\%)$ & 28.1 & 30.8 & 58.6 & 45.3 \\
\hline
\end{tabular}


Appendix G. Water contact angles of extracted and unextracted yellow-poplar samples.

\begin{tabular}{|c|c|c|c|c|c|c|c|c|c|}
\hline \multirow{2}{*}{$\begin{array}{c}\text { Wood } \\
\text { Species }\end{array}$} & \multirow{2}{*}{$\begin{array}{c}\text { Surface } \\
\text { Treatment }\end{array}$} & \multirow{2}{*}{$\begin{array}{l}\text { Number of } \\
\text { Replication }\end{array}$} & \multicolumn{7}{|c|}{ Contact Angle ( $\left.{ }^{\circ}\right)$} \\
\hline & & & $0 \mathrm{~s}$. & $10 \mathrm{~s}$. & $20 \mathrm{~s}$. & $30 \mathrm{~s}$. & $40 \mathrm{~s}$. & $50 \mathrm{~s}$. & $60 \mathrm{~s}$. \\
\hline \multirow[t]{14}{*}{ Yellow-Poplar } & \multirow[t]{14}{*}{ YP50EXT } & 1 & 58.5 & 53.0 & 52.3 & 52.3 & 52.3 & 49.9 & 49.9 \\
\hline & & 2 & 63.2 & 56.0 & 53.5 & 51.8 & 51.8 & 51.0 & 51.0 \\
\hline & & 3 & 61.6 & 60.1 & 59.2 & 58.2 & 57.3 & 57.3 & 55.3 \\
\hline & & 4 & 56.7 & 54.0 & 42.7 & 40.6 & 39.5 & 39.5 & 39.5 \\
\hline & & 5 & 51.3 & 43.4 & 40.5 & 39.3 & 38.3 & 38.3 & 37.8 \\
\hline & & 6 & 61.4 & 56.6 & 48.7 & 47.2 & 47.2 & 47.2 & 46.8 \\
\hline & & 7 & 59.2 & 57.6 & 56.3 & 55.6 & 53.2 & 53.2 & 52.2 \\
\hline & & 8 & 56.0 & 44.2 & 38.0 & 36.8 & 35.7 & 35.7 & 34.6 \\
\hline & & 9 & 51.7 & 40.7 & 35.4 & 34.3 & 32.0 & 30.6 & 30.1 \\
\hline & & 10 & 57.1 & 51.2 & 48.9 & 48.0 & 47.0 & 44.8 & 44.8 \\
\hline & & & 10 & 10 & 10 & 10 & 10 & 10 & 10 \\
\hline & & AVERAGE & $\mathbf{5 7 . 7}$ & 51.7 & 47.6 & 46.4 & 45.4 & 44.8 & 44.2 \\
\hline & & STDEV & 4.0 & 6.7 & 8.1 & 8.3 & 8.5 & 8.5 & 8.4 \\
\hline & & $\operatorname{COV}(\%)$ & 6.9 & 12.9 & 16.9 & 17.8 & 18.8 & 19.0 & 18.9 \\
\hline \multirow[t]{14}{*}{ Yellow-Poplar } & \multirow[t]{14}{*}{ YP200 } & 1 & 96.7 & 95.5 & 95.5 & 93.1 & 90.1 & 88.3 & 88.3 \\
\hline & & 2 & 95.3 & 94.1 & 94.1 & 94.1 & 93.3 & 90.7 & 89.2 \\
\hline & & 3 & 102.6 & 97.1 & 96.1 & 96.1 & 93.6 & 90.6 & 90.6 \\
\hline & & 4 & 99.3 & 93.9 & 93.9 & 89.2 & 89.2 & 87.2 & 86.3 \\
\hline & & 5 & 100.7 & 95.9 & 95.9 & 95.9 & 95.9 & 91.7 & 91.7 \\
\hline & & 6 & 97.2 & 80.8 & 79.8 & 79.8 & 79.8 & 78.6 & 78.6 \\
\hline & & 7 & 87.2 & 85.1 & 83.2 & 80.1 & 77.3 & 76.2 & 75.2 \\
\hline & & 8 & 96.6 & 96.6 & 94.0 & 91.5 & 88.1 & 88.1 & 86.3 \\
\hline & & 9 & 97.0 & 94.2 & 92.3 & 92.3 & 92.3 & 92.3 & 91.8 \\
\hline & & 10 & 84.5 & 82.5 & 82.5 & 82.5 & 80.6 & 80.6 & 79.9 \\
\hline & & & 10 & 10 & 10 & 10 & 10 & 10 & 10 \\
\hline & & AVERAGE & 95.7 & 91.6 & 90.7 & 89.5 & 88.0 & 86.4 & 85.8 \\
\hline & & STDEV & 5.7 & 6.2 & 6.3 & 6.3 & 6.5 & 5.8 & 5.9 \\
\hline & & $\operatorname{COV}(\%)$ & 5.9 & 6.8 & 6.9 & 7.1 & 7.4 & 6.7 & 6.9 \\
\hline \multirow[t]{14}{*}{ Yellow-Poplar } & \multirow[t]{14}{*}{ YPEXT200 } & 1 & 82.2 & 81.7 & 81.7 & 81.7 & 81.7 & 81.7 & 81.7 \\
\hline & & 2 & 105.9 & 100.4 & 98.4 & 95.7 & 91.8 & 90.7 & 90.7 \\
\hline & & 3 & 103.2 & 102.1 & 99.8 & 99.2 & 99.2 & 99.2 & 99.2 \\
\hline & & 4 & 99.5 & 96.1 & 96.1 & 95.1 & 95.1 & 93.5 & 93.5 \\
\hline & & 5 & 102.0 & 102.0 & 99.9 & 99.9 & 99.9 & 99.9 & 99.9 \\
\hline & & 6 & 91.5 & 87.3 & 85.0 & 83.3 & 81.7 & 81.7 & 81.7 \\
\hline & & 7 & 94.2 & 93.1 & 93.1 & 93.1 & 93.1 & 92.2 & 92.2 \\
\hline & & 8 & 99.1 & 96.8 & 95.5 & 90.3 & 89.3 & 88.0 & 88.0 \\
\hline & & 9 & 106.3 & 106.3 & 106.3 & 101.3 & 99.3 & 99.3 & 99.3 \\
\hline & & 10 & 94.6 & 93.5 & 93.5 & 93.5 & 93.5 & 91.5 & 91.5 \\
\hline & & $\mathrm{n}$ & 10 & 10 & 10 & 10 & 10 & 10 & 10 \\
\hline & & AVERAGE & 97.9 & 95.9 & 94.9 & 93.3 & 92.5 & 91.8 & 91.8 \\
\hline & & STDEV & 7.4 & 7.4 & 7.2 & 6.6 & 6.7 & 6.7 & 6.7 \\
\hline & & $\operatorname{COV}(\%)$ & 7.6 & 7.7 & 7.6 & 7.1 & 7.2 & 7.3 & 7.3 \\
\hline \multirow[t]{14}{*}{ Yellow-Poplar } & \multirow[t]{14}{*}{ YP200EXT } & 1 & 71.5 & 69.2 & 66.1 & 65.4 & 64.2 & 63.9 & 63.9 \\
\hline & & 2 & 74.6 & 69.4 & 65.2 & 64.0 & 63.5 & 62.9 & 62.9 \\
\hline & & 3 & 70.2 & 66.4 & 64.7 & 61.4 & 60.1 & 59.9 & 59.5 \\
\hline & & 4 & 72.5 & 70.8 & 68.2 & 67.7 & 67.7 & 67.7 & 67.7 \\
\hline & & 5 & 73.8 & 70.0 & 69.2 & 68.4 & 68.4 & 68.4 & 68.4 \\
\hline & & 6 & 71.8 & 66.2 & 65.5 & 64.4 & 64.4 & 60.2 & 60.2 \\
\hline & & 7 & 75.0 & 65.4 & 65.4 & 62.6 & 61.2 & 59.6 & 59.6 \\
\hline & & 8 & 68.1 & 56.6 & 53.4 & 52.6 & 52.6 & 51.1 & 51.1 \\
\hline & & 9 & 65.6 & 58.5 & 57.1 & 55.7 & 54.9 & 51.7 & 51.7 \\
\hline & & 10 & 75.1 & 67.2 & 65.0 & 60.3 & 58.6 & 58.6 & 58.6 \\
\hline & & & 10 & 10 & 10 & 10 & 10 & 10 & 10 \\
\hline & & AVERAGE & 71.8 & 66.0 & 64.0 & 62.2 & 61.5 & 60.4 & 60.3 \\
\hline & & STDEV & 3.1 & 4.8 & 4.9 & 5.0 & 5.1 & 5.8 & 5.8 \\
\hline & & $\operatorname{COV}(\%)$ & 4.3 & 7.3 & 7.6 & 8.1 & 8.4 & 9.6 & 9.6 \\
\hline
\end{tabular}


Appendix $H$. Water contact angle of extracted and unextracted southern pine samples.

\begin{tabular}{|c|c|c|c|c|c|c|c|c|c|}
\hline \multirow{2}{*}{$\begin{array}{c}\text { Wood } \\
\text { Species }\end{array}$} & \multirow{2}{*}{$\begin{array}{c}\text { Surface } \\
\text { Treatment }\end{array}$} & \multirow{2}{*}{$\begin{array}{l}\text { Number of } \\
\text { Replication }\end{array}$} & \multicolumn{7}{|c|}{ Contact Angle $\left(^{\circ}\right)$} \\
\hline & & & $0 \mathrm{~s}$. & $10 \mathrm{~s}$. & $20 \mathrm{~s}$. & $30 \mathrm{~s}$. & $40 \mathrm{~s}$. & $50 \mathrm{~s}$. & $60 \mathrm{~s}$. \\
\hline \multirow[t]{14}{*}{ Southern Pine } & \multirow[t]{14}{*}{ SP50EXT } & 1 & 87.5 & 85.0 & 81.9 & 75.9 & 71.1 & 62.1 & 56.1 \\
\hline & & 2 & 78.3 & 76.2 & 73.1 & 72.6 & 72.6 & 70.4 & 69.6 \\
\hline & & 3 & 83.0 & 76.7 & 75.1 & 72.4 & 71.2 & 69.4 & 69.0 \\
\hline & & 4 & 83.4 & 82.7 & 82.7 & 79.5 & 76.6 & 76.6 & 75.6 \\
\hline & & 5 & 82.0 & 74.8 & 68.4 & 67.1 & 66.1 & 66.1 & 64.6 \\
\hline & & 6 & 78.6 & 77.6 & 77.6 & 77.6 & 77.6 & 75.8 & 74.9 \\
\hline & & 7 & 77.5 & 65.0 & 60.0 & 60.0 & 58.6 & 58.6 & 58.6 \\
\hline & & 8 & 86.1 & 77.5 & 76.2 & 74.7 & 74.7 & 74.7 & 73.8 \\
\hline & & 9 & 84.1 & 75.5 & 73.7 & 73.7 & 73.7 & 71.1 & 69.3 \\
\hline & & 10 & 74.2 & 70.6 & 69.6 & 68.4 & 67.6 & 67.6 & 67.6 \\
\hline & & & 10 & 10 & 10 & 10 & 10 & 10 & 10 \\
\hline & & AVERAGE & 81.5 & 76.2 & 73.8 & 72.2 & 71.0 & 69.2 & 67.9 \\
\hline & & STDEV & 4.2 & 5.6 & 6.7 & 5.7 & 5.7 & 5.9 & 6.6 \\
\hline & & $\operatorname{COV}(\%)$ & 5.1 & 7.4 & 9.1 & 7.9 & 8.0 & 8.5 & 9.7 \\
\hline \multirow[t]{14}{*}{ Southern Pine } & \multirow[t]{14}{*}{ SP200 } & 1 & 110.3 & 110.3 & 108.3 & 105.3 & 105.3 & 105.3 & 105.3 \\
\hline & & 2 & 117.9 & 117.9 & 115.3 & 115.3 & 115.3 & 113.3 & 113.3 \\
\hline & & 3 & 96.7 & 90.8 & 88.2 & 85.0 & 82.7 & 81.3 & 80.4 \\
\hline & & 4 & 97.5 & 81.3 & 75.9 & 74.1 & 74.1 & 74.1 & 73.1 \\
\hline & & 5 & 106.7 & 99.8 & 99.8 & 98.3 & 96.5 & 89.6 & 84.7 \\
\hline & & 6 & 107.4 & 101.7 & 92.4 & 87.4 & 83.8 & 82.8 & 82.8 \\
\hline & & 7 & 91.8 & 83.6 & 81.7 & 80.6 & 80.6 & 79.1 & 78.1 \\
\hline & & 8 & 93.6 & 90.7 & 89.3 & 88.3 & 88.3 & 88.3 & 87.3 \\
\hline & & 9 & 88.6 & 82.2 & 78.4 & 69.3 & 67.2 & 67.2 & 67.2 \\
\hline & & 10 & 90.5 & 83.3 & 76.8 & 76.0 & 76.0 & 72.4 & 72.4 \\
\hline & & & 10 & 10 & 10 & 10 & 10 & 10 & 10 \\
\hline & & AVERAGE & 100.1 & 94.1 & 90.6 & 88.0 & 87.0 & 85.3 & 84.5 \\
\hline & & STDEV & 9.8 & 12.8 & 13.5 & 14.5 & 14.8 & 14.5 & 14.5 \\
\hline & & $\operatorname{COV}(\%)$ & 9.8 & 13.6 & 14.9 & 16.5 & 17.0 & 17.0 & 17.2 \\
\hline \multirow[t]{14}{*}{ Southern Pine } & \multirow[t]{14}{*}{ SPEXT200 } & 1 & 94.7 & 93.7 & 93.7 & 93.7 & 93.7 & 93.7 & 93.7 \\
\hline & & 2 & 97.5 & 97.5 & 95.7 & 95.7 & 95.7 & 94.7 & 94.7 \\
\hline & & 3 & 116.5 & 116.5 & 116.5 & 116.5 & 116.5 & 114.3 & 114.3 \\
\hline & & 4 & 111.5 & 111.5 & 111.5 & 109.4 & 109.4 & 109.4 & 109.4 \\
\hline & & 5 & 119.7 & 119.7 & 119.7 & 119.7 & 119.7 & 119.7 & 119.7 \\
\hline & & 6 & 106.7 & 93.1 & 93.1 & 93.1 & 92.2 & 92.2 & 92.2 \\
\hline & & 7 & 114.4 & 112.3 & 111.2 & 111.2 & 111.2 & 110.1 & 110.1 \\
\hline & & 8 & 111.2 & 110.1 & 110.1 & 110.1 & 110.1 & 110.1 & 110.1 \\
\hline & & 9 & 104.9 & 104.9 & 104.9 & 104.9 & 104.9 & 102.2 & 100.8 \\
\hline & & 10 & 113.8 & 113.8 & 113.8 & 110.4 & 110.4 & 110.4 & 107.6 \\
\hline & & & 10 & 10 & 10 & 10 & 10 & 10 & 10 \\
\hline & & AVERAGE & 109.1 & 107.3 & 107.0 & 106.5 & 106.4 & 105.7 & 105.2 \\
\hline & & STDEV & 8.1 & 9.5 & 9.7 & 9.4 & 9.5 & 9.4 & 9.4 \\
\hline & & $\operatorname{COV}(\%)$ & 7.4 & 8.9 & 9.1 & 8.8 & 9.0 & 8.9 & 8.9 \\
\hline \multirow[t]{14}{*}{ Southern Pine } & \multirow[t]{14}{*}{ SP200EXT } & 1 & 92.1 & 89.9 & 85.7 & 84.2 & 83.3 & 80.1 & 80.1 \\
\hline & & 2 & 99.5 & 99.5 & 96.3 & 91.7 & 90.2 & 89.1 & 89.1 \\
\hline & & 3 & 86.5 & 85.2 & 85.2 & 82.4 & 80.1 & 80.1 & 80.1 \\
\hline & & 4 & 87.5 & 85.1 & 78.9 & 78.9 & 78.9 & 78.9 & 78.9 \\
\hline & & 5 & 86.7 & 86.7 & 86.7 & 86.7 & 85.1 & 85.1 & 84.5 \\
\hline & & 6 & 92.0 & 87.1 & 85.7 & 85.7 & 85.7 & 83.9 & 83.9 \\
\hline & & 7 & 87.3 & 79.4 & 79.4 & 78.6 & 78.6 & 78.6 & 78.6 \\
\hline & & 8 & 97.2 & 97.2 & 94.9 & 94.9 & 92.1 & 90.2 & 89.4 \\
\hline & & 9 & 102.2 & 97.3 & 95.2 & 89.5 & 88.2 & 88.2 & 88.2 \\
\hline & & 10 & 90.8 & 78.2 & 78.2 & 74.1 & 70.9 & 70.9 & 70.9 \\
\hline & & $\mathrm{n}$ & 10 & 10 & 10 & 10 & 10 & 10 & 10 \\
\hline & & AVERAGE & 92.2 & 88.6 & 86.6 & 84.7 & 83.3 & 82.5 & 82.4 \\
\hline & & STDEV & 5.7 & 7.4 & 6.8 & 6.4 & 6.4 & 6.0 & 5.8 \\
\hline & & $\operatorname{COV}(\%)$ & 6.1 & 8.3 & 7.9 & 7.6 & 7.6 & 7.2 & 7.1 \\
\hline
\end{tabular}


Appendix I. Strain energy release rate results of yellow-poplar and southern pine specimens bonded with phenol-formaldehyde adhesive.

\begin{tabular}{|c|c|c|c|c|}
\hline \multirow{2}{*}{$\begin{array}{c}\text { Wood } \\
\text { Species }\end{array}$} & \multirow{2}{*}{$\begin{array}{c}\text { Surface } \\
\text { Treatment }\end{array}$} & \multirow{2}{*}{$\begin{array}{l}\text { Number of } \\
\text { Replication }\end{array}$} & \multicolumn{2}{|c|}{ Strain Energy Release Rate $\left(\mathrm{J} / \mathrm{m}^{2}\right)$} \\
\hline & & & MAX & ARR \\
\hline Southern Pine & SP200 & $\begin{array}{r}1 \\
2 \\
3 \\
4 \\
\mathrm{n} \\
\text { AVERAGE } \\
\text { STDEV } \\
\operatorname{COV}(\%)\end{array}$ & $\begin{array}{r}92.7 \\
71.2 \\
102.0 \\
97.6 \\
4 \\
\mathbf{9 0 . 9} \\
13.6 \\
15.0\end{array}$ & $\begin{array}{r}59.1 \\
53.4 \\
46.1 \\
63.3 \\
4 \\
\mathbf{5 5 . 5} \\
7.4 \\
13.4\end{array}$ \\
\hline Southern Pine & SP200EXT & $\begin{array}{r}1 \\
2 \\
3 \\
4 \\
\mathrm{n} \\
\text { AVERAGE } \\
\text { STDEV } \\
\operatorname{COV}(\%)\end{array}$ & \begin{tabular}{r|}
125.0 \\
236.3 \\
326.2 \\
76.6 \\
4 \\
191.0 \\
112.2 \\
58.7
\end{tabular} & $\begin{array}{r}61.2 \\
177.6 \\
278.6 \\
51.3 \\
4 \\
142.2 \\
107.5 \\
75.6\end{array}$ \\
\hline Southern Pine & SPEXT200 & $\begin{array}{r}1 \\
2 \\
3 \\
4 \\
n \\
\text { AVERAGE } \\
\text { STDEV } \\
\operatorname{COV}(\%) \\
\end{array}$ & \begin{tabular}{r|}
142.9 \\
142.2 \\
152.5 \\
151.1 \\
4 \\
$\mathbf{1 4 7 . 1}$ \\
5.4 \\
3.7 \\
\end{tabular} & $\begin{array}{r}124.7 \\
121.7 \\
132.3 \\
137.8 \\
4 \\
\mathbf{1 2 9 . 1} \\
7.3 \\
5.6\end{array}$ \\
\hline Yellow-Poplar & YP200 & $\begin{array}{r}1 \\
2 \\
3 \\
4 \\
\mathrm{n} \\
\text { AVERAGE } \\
\text { STDEV } \\
\text { COV }(\%)\end{array}$ & \begin{tabular}{r|r|}
397.9 \\
485.3 \\
467.9 \\
233.7 \\
4 \\
$\mathbf{3 9 6 . 2}$ \\
114.7 \\
29.0
\end{tabular} & $\begin{array}{r}297.7 \\
361.5 \\
433.2 \\
187.4 \\
4 \\
\mathbf{3 1 9 . 9} \\
104.3 \\
32.6\end{array}$ \\
\hline Yellow-Poplar & YP200EXT & \begin{tabular}{r|}
1 \\
2 \\
3 \\
4 \\
$\mathrm{n}$ \\
AVERAGE \\
STDEV \\
$\operatorname{COV}(\%)$
\end{tabular} & $\begin{array}{r}434.2 \\
547.9 \\
485.6 \\
453.2 \\
4 \\
\mathbf{4 8 0 . 2} \\
49.9 \\
10.4\end{array}$ & $\begin{array}{r}312.4 \\
497.4 \\
431.9 \\
393.4 \\
4 \\
\mathbf{4 0 8 . 8} \\
77.3 \\
18.9\end{array}$ \\
\hline
\end{tabular}


Appendix J. Water contact angles of treated southern pine surfaces.

\begin{tabular}{|c|c|c|c|c|c|c|c|c|c|}
\hline \multirow{2}{*}{$\begin{array}{c}\text { Wood } \\
\text { Species }\end{array}$} & \multirow{2}{*}{$\begin{array}{c}\text { Surface } \\
\text { Treatment }\end{array}$} & \multirow{2}{*}{$\begin{array}{l}\text { Number of } \\
\text { Replication }\end{array}$} & \multicolumn{7}{|c|}{ Contact Angle ( ${ }^{0}$ ) } \\
\hline & & & $0 \mathrm{~s}$. & $10 \mathrm{~s}$. & $20 \mathrm{~s}$. & $30 \mathrm{~s}$. & $40 \mathrm{~s}$. & $50 \mathrm{~s}$. & $60 \mathrm{~s}$. \\
\hline \multirow[t]{16}{*}{ Southern Pine } & \multirow[t]{16}{*}{ SPC } & 1 & 44.9 & 0.0 & 0.0 & 0.0 & 0.0 & 0.0 & 0.0 \\
\hline & & 2 & 39.0 & 7.2 & 0.0 & 0.0 & 0.0 & 0.0 & 0.0 \\
\hline & & 3 & 43.9 & 11.3 & 2.7 & 0.0 & 0.0 & 0.0 & 0.0 \\
\hline & & 4 & 46.4 & 7.6 & 0.0 & 0.0 & 0.0 & 0.0 & 0.0 \\
\hline & & 5 & 44.3 & 15.2 & 7.5 & 4.2 & 0.0 & 0.0 & 0.0 \\
\hline & & 6 & 44.4 & 19.4 & 17.2 & 14.7 & 9.3 & 7.1 & 4.1 \\
\hline & & 7 & 47.2 & 20.3 & 15.7 & 14.1 & 11.9 & 9.1 & 8.1 \\
\hline & & 8 & 46.4 & 18.0 & 12.1 & 10.0 & 8.5 & 9.3 & 6.0 \\
\hline & & 9 & 46.8 & 14.0 & 13.0 & 11.9 & 9.5 & 5.7 & 3.6 \\
\hline & & 10 & 49.2 & 28.1 & 22.1 & 18.6 & 15.3 & 13.2 & 11.9 \\
\hline & & 11 & 48.2 & 9.1 & 0.0 & 0.0 & 0.0 & 0.0 & 0.0 \\
\hline & & 12 & 47.5 & 8.2 & 0.0 & 0.0 & 0.0 & 0.0 & 0.0 \\
\hline & & & 12 & 12 & 12 & 12 & 12 & 12 & 12 \\
\hline & & AVERAGE & 45.7 & 13.2 & 7.5 & 6.1 & 4.5 & 3.7 & 2.8 \\
\hline & & STDEV & 2.7 & 7.6 & 8.2 & 7.2 & 5.9 & 4.9 & 4.0 \\
\hline & & $\operatorname{COV}(\%)$ & 5.9 & 57.2 & 108.2 & 117.5 & 129.0 & 131.8 & 143.3 \\
\hline \multirow[t]{16}{*}{ Southern Pine } & \multirow[t]{16}{*}{ SPI } & 1 & 100.0 & 97.9 & 97.9 & 97.9 & 97.9 & 97.9 & 97.9 \\
\hline & & 2 & 107.4 & 105.8 & 105.8 & 105.8 & 103.8 & 103.8 & 103.8 \\
\hline & & 3 & 98.4 & 95.7 & 95.7 & 95.7 & 94.3 & 94.3 & 94.3 \\
\hline & & 4 & 100.6 & 100.6 & 100.6 & 100.6 & 100.6 & 100.6 & 100.6 \\
\hline & & 5 & 100.3 & 99.8 & 99.8 & 99.8 & 98.7 & 98.7 & 98.7 \\
\hline & & 6 & 101.6 & 100.5 & 99.2 & 98.1 & 98.1 & 98.1 & 98.1 \\
\hline & & 7 & 101.8 & 100.0 & 94.5 & 93.4 & 93.4 & 93.4 & 93.4 \\
\hline & & 8 & 100.6 & 98.6 & 98.6 & 98.6 & 96.6 & 96.6 & 96.6 \\
\hline & & 9 & 100.2 & 100.2 & 100.2 & 99.5 & 99.5 & 97.6 & 97.6 \\
\hline & & 10 & 97.4 & 95.7 & 94.6 & 94.6 & 94.6 & 94.6 & 94.6 \\
\hline & & 11 & 102.2 & 100.5 & 98.2 & 98.2 & 98.2 & 93.5 & 93.5 \\
\hline & & 12 & 99.6 & 98.8 & 95.8 & 95.8 & 94.9 & 94.9 & 94.9 \\
\hline & & & 12 & 12 & 12 & 12 & 12 & 12 & 12 \\
\hline & & AVERAGE & 100.8 & 99.5 & 98.4 & 98.2 & 97.6 & 97.0 & 97.0 \\
\hline & & STDEV & 2.5 & 2.6 & 3.2 & 3.3 & 3.0 & 3.1 & 3.1 \\
\hline & & $\operatorname{COV}(\%)$ & 2.5 & 2.7 & 3.2 & 3.3 & 3.1 & 3.2 & 3.2 \\
\hline \multirow{16}{*}{ Southern Pine } & \multirow[t]{16}{*}{ SPIHMR } & 1 & 30.0 & 6.1 & 0.0 & 0.0 & 0.0 & 0.0 & 0.0 \\
\hline & & 2 & 25.0 & 0.0 & 0.0 & 0.0 & 0.0 & 0.0 & 0.0 \\
\hline & & 3 & 29.7 & 0.0 & 0.0 & 0.0 & 0.0 & 0.0 & 0.0 \\
\hline & & 4 & 21.9 & 0.0 & 0.0 & 0.0 & 0.0 & 0.0 & 0.0 \\
\hline & & 5 & 21.8 & 0.0 & 0.0 & 0.0 & 0.0 & 0.0 & 0.0 \\
\hline & & 6 & 28.4 & 0.0 & 0.0 & 0.0 & 0.0 & 0.0 & 0.0 \\
\hline & & 7 & 21.0 & 0.0 & 0.0 & 0.0 & 0.0 & 0.0 & 0.0 \\
\hline & & 8 & 21.9 & 0.0 & 0.0 & 0.0 & 0.0 & 0.0 & 0.0 \\
\hline & & 9 & 38.2 & 6.5 & 0.0 & 0.0 & 0.0 & 0.0 & 0.0 \\
\hline & & 10 & 39.7 & 6.4 & 0.0 & 0.0 & 0.0 & 0.0 & 0.0 \\
\hline & & 11 & 41.4 & 8.3 & 6.3 & 0.0 & 0.0 & 0.0 & 0.0 \\
\hline & & 12 & 41.8 & 14.8 & 8.1 & 0.0 & 0.0 & 0.0 & 0.0 \\
\hline & & $\mathrm{n}$ & 12 & 12 & 12 & 12 & 12 & 12 & 12 \\
\hline & & AVERAGE & 30.0 & 3.5 & 1.2 & 0.0 & 0.0 & 0.0 & 0.0 \\
\hline & & STDEV & 8.2 & 4.9 & 2.8 & 0.0 & 0.0 & 0.0 & 0.0 \\
\hline & & $\operatorname{COV}(\%)$ & 27.2 & 138.8 & 235.7 & 0.0 & 0.0 & 0.0 & 0.0 \\
\hline
\end{tabular}


Appendix K. Water contact angles of treated southern pine surfaces.

\begin{tabular}{|c|c|c|c|c|c|c|c|c|c|}
\hline \multirow{2}{*}{$\begin{array}{c}\text { Wood } \\
\text { Species }\end{array}$} & \multirow{2}{*}{$\begin{array}{c}\text { Surface } \\
\text { Treatment }\end{array}$} & \multirow{2}{*}{$\begin{array}{l}\text { Number of } \\
\text { Replication }\end{array}$} & \multicolumn{7}{|c|}{ Contact Angle $\left({ }^{\circ}\right)$} \\
\hline & & & $0 \mathrm{~s}$. & $10 \mathrm{~s}$. & $20 \mathrm{~s}$. & $30 \mathrm{~s}$. & $40 \mathrm{~s}$. & $50 \mathrm{~s}$. & $60 \mathrm{~s}$. \\
\hline \multirow{16}{*}{ Southern Pine } & \multirow[t]{16}{*}{$\begin{array}{l}\text { SPIXY } \\
\end{array}$} & 1 & 90.0 & 41.5 & 35.6 & 34.0 & 33.2 & 32.7 & 25.5 \\
\hline & & 2 & 83.4 & 68.9 & 66.1 & 59.4 & 56.7 & 51.4 & 47.9 \\
\hline & & 3 & 85.0 & 63.1 & 54.1 & 54.1 & 53.3 & 51.2 & 50.5 \\
\hline & & 4 & 83.2 & 63.1 & 57.9 & 53.7 & 50.9 & 48.6 & 44.0 \\
\hline & & 5 & 89.6 & 67.5 & 60.4 & 58.3 & 56.5 & 54.0 & 50.7 \\
\hline & & 6 & 84.4 & 71.5 & 69.0 & 67.5 & 65.6 & 63.6 & 61.1 \\
\hline & & 7 & 87.1 & 70.9 & 66.1 & 57.0 & 55.9 & 54.7 & 52.6 \\
\hline & & 8 & 84.7 & 58.4 & 40.1 & 35.5 & 34.0 & 32.3 & 31.9 \\
\hline & & 9 & 80.4 & 65.1 & 44.8 & 43.1 & 37.7 & 36.8 & 34.3 \\
\hline & & 10 & 84.0 & 69.2 & 68.3 & 65.4 & 61.4 & 59.9 & 56.8 \\
\hline & & 11 & 80.3 & 65.7 & 64.0 & 62.4 & 62.2 & 61.4 & 59.8 \\
\hline & & 12 & 80.0 & 74.2 & 61.5 & 57.6 & 53.9 & 51.4 & 48.5 \\
\hline & & & 12 & 12 & 12 & 12 & 12 & 12 & 12 \\
\hline & & AVERAGE & 84.3 & 64.9 & 57.3 & 54.0 & 51.8 & 49.8 & 47.0 \\
\hline & & STDEV & 3.3 & 8.6 & 11.3 & 10.9 & 11.0 & 10.6 & 11.2 \\
\hline & & $\operatorname{COV}(\%)$ & 3.9 & 13.2 & 19.8 & 20.2 & 21.2 & 21.3 & 23.8 \\
\hline \multirow[t]{16}{*}{ Southern Pine } & \multirow[t]{16}{*}{ SPINA } & 1 & 59.9 & 18.2 & 0.0 & 0.0 & 0.0 & 0.0 & 0.0 \\
\hline & & 2 & 60.1 & 16.0 & 8.7 & 0.0 & 0.0 & 0.0 & 0.0 \\
\hline & & 3 & 48.7 & 0.0 & 0.0 & 0.0 & 0.0 & 0.0 & 0.0 \\
\hline & & 4 & 49.4 & 10.9 & 0.0 & 0.0 & 0.0 & 0.0 & 0.0 \\
\hline & & 5 & 52.1 & 7.7 & 0.0 & 0.0 & 0.0 & 0.0 & 0.0 \\
\hline & & 6 & 49.8 & 17.0 & 4.2 & 0.0 & 0.0 & 0.0 & 0.0 \\
\hline & & 7 & 51.3 & 4.6 & 0.0 & 0.0 & 0.0 & 0.0 & 0.0 \\
\hline & & 8 & 49.6 & 4.0 & 0.0 & 0.0 & 0.0 & 0.0 & 0.0 \\
\hline & & 9 & 47.3 & 0.0 & 0.0 & 0.0 & 0.0 & 0.0 & 0.0 \\
\hline & & 10 & 60.7 & 19.9 & 13.0 & 0.0 & 0.0 & 0.0 & 0.0 \\
\hline & & 11 & 50.6 & 16.0 & 9.8 & 0.0 & 0.0 & 0.0 & 0.0 \\
\hline & & 12 & 59.7 & 31.3 & 20.8 & 0.0 & 0.0 & 0.0 & 0.0 \\
\hline & & & 12 & 12 & 12 & 12 & 12 & 12 & 12 \\
\hline & & AVERAGE & 53.3 & 12.1 & 4.7 & 0.0 & 0.0 & 0.0 & 0.0 \\
\hline & & STDEV & 5.2 & 9.3 & 6.9 & 0.0 & 0.0 & 0.0 & 0.0 \\
\hline & & $\operatorname{COV}(\%)$ & 9.8 & 76.9 & 146.8 & 0.0 & 0.0 & 0.0 & 0.0 \\
\hline \multirow{16}{*}{ Southern Pine } & \multirow[t]{16}{*}{ SPIXYNA } & 1 & 78.7 & 31.1 & 21.4 & 12.1 & 6.8 & 3.4 & 0.0 \\
\hline & & 2 & 69.2 & 18.1 & 13.0 & 5.2 & 3.1 & 0.0 & 0.0 \\
\hline & & 3 & 67.9 & 13.2 & 5.5 & 3.1 & 0.0 & 0.0 & 0.0 \\
\hline & & 4 & 67.3 & 38.7 & 25.2 & 21.6 & 17.4 & 15.6 & 10.1 \\
\hline & & 5 & 80.7 & 50.1 & 40.4 & 28.4 & 23.1 & 21.1 & 16.7 \\
\hline & & 6 & 71.1 & 7.7 & 0.0 & 0.0 & 0.0 & 0.0 & 0.0 \\
\hline & & 7 & 74.2 & 16.0 & 3.3 & 0.0 & 0.0 & 0.0 & 0.0 \\
\hline & & 8 & 75.0 & 29.8 & 15.0 & 0.0 & 0.0 & 0.0 & 0.0 \\
\hline & & 9 & 71.7 & 16.1 & 0.0 & 0.0 & 0.0 & 0.0 & 0.0 \\
\hline & & 10 & 67.8 & 26.0 & 22.4 & 16.0 & 12.7 & 10.7 & 7.6 \\
\hline & & 11 & 76.8 & 28.8 & 18.3 & 10.7 & 5.5 & 0.0 & 0.0 \\
\hline & & 12 & 68.0 & 29.5 & 20.4 & 18.4 & 14.5 & 12.6 & 9.2 \\
\hline & & $\mathrm{n}$ & 12 & 12 & 12 & 12 & 12 & 12 & 12 \\
\hline & & AVERAGE & 72.4 & 25.4 & 15.4 & 9.6 & 6.9 & 5.3 & 3.6 \\
\hline & & STDEV & 4.6 & 11.9 & 11.9 & 9.8 & 8.1 & 7.6 & 5.8 \\
\hline & & $\operatorname{COV}(\%)$ & 6.4 & 46.8 & 77.5 & 101.6 & 116.6 & 144.2 & 158.4 \\
\hline
\end{tabular}


Appendix L. Strain energy release rate of treated southern pine bonded with PF, PVA, PFHMR, and PMDI adhesives.

\begin{tabular}{|c|c|c|c|c|c|c|c|c|c|c|}
\hline \multicolumn{3}{|c|}{ MAX $=$ Maximum, ARR $=$ Arrested } & \multicolumn{8}{|c|}{ Strain Energy Release Rate $\left(\mathrm{J} / \mathrm{m}^{2}\right)$} \\
\hline \multirow{2}{*}{$\begin{array}{c}\text { Wood } \\
\text { Species }\end{array}$} & \multirow{2}{*}{$\begin{array}{c}\text { Surface } \\
\text { Treatment }\end{array}$} & \multirow{2}{*}{$\begin{array}{l}\text { Number of } \\
\text { Replication }\end{array}$} & \multicolumn{2}{|c|}{$\begin{array}{l}\text { PVA } \\
\end{array}$} & \multicolumn{2}{|c|}{$\mathbf{P F}$} & \multicolumn{2}{|c|}{ PFHMR } & \multicolumn{2}{|c|}{ PMDI } \\
\hline & & & MAX & ARR & MAX & ARR & MAX & ARR & MAX & $\mathbf{A R R}$ \\
\hline Southern Pine & SPC & \begin{tabular}{r|}
1 \\
2 \\
3 \\
4 \\
5 \\
6 \\
\\
$\mathrm{n}$ \\
n \\
AVERAGE \\
STDEV \\
COV (\%)
\end{tabular} & $\begin{array}{r}260.2 \\
187.0 \\
316.5 \\
232.6 \\
330.6 \\
253.0 \\
330.8 \\
171.6 \\
8 \\
\mathbf{2 6 0 . 3} \\
62.3 \\
23.9\end{array}$ & $\begin{array}{r}243.8 \\
181.9 \\
287.4 \\
212.0 \\
294.3 \\
229.8 \\
291.5 \\
143.8 \\
8 \\
\mathbf{2 3 5 . 6} \\
55.1 \\
23.4\end{array}$ & $\begin{array}{r}276.7 \\
269.1 \\
264.9 \\
203.0 \\
214.7 \\
271.9 \\
260.2 \\
226.3 \\
8 \\
\mathbf{2 4 8 . 3} \\
29.0 \\
11.7\end{array}$ & $\begin{array}{r}253.8 \\
250.7 \\
245.8 \\
182.4 \\
190.5 \\
248.9 \\
223.1 \\
222.4 \\
8 \\
\mathbf{2 2 7 . 2} \\
28.0 \\
12.3\end{array}$ & & & & \\
\hline Southern Pine & SPI & $\begin{array}{r}1 \\
2 \\
3 \\
4 \\
5 \\
6 \\
\\
\mathrm{n} \\
\text { AVERAGE } \\
\text { STDEV } \\
\operatorname{COV}(\%)\end{array}$ & \begin{tabular}{r|}
30.0 \\
41.6 \\
51.7 \\
65.5 \\
52.3 \\
47.4 \\
76.7 \\
38.2 \\
8 \\
$\mathbf{5 0 . 4}$ \\
15.0 \\
29.8
\end{tabular} & \begin{tabular}{r|}
17.3 \\
33.7 \\
38.3 \\
49.8 \\
39.6 \\
39.0 \\
49.3 \\
24.4 \\
8 \\
36.4 \\
11.2 \\
30.8
\end{tabular} & $\begin{array}{r}44.1 \\
84.4 \\
125.1 \\
60.5 \\
26.1 \\
45.1 \\
39.3 \\
64.2 \\
8 \\
\mathbf{6 1 . 1} \\
31.4 \\
51.3\end{array}$ & $\begin{array}{r}26.4 \\
71.2 \\
83.8 \\
55.1 \\
18.7 \\
36.7 \\
30.0 \\
52.8 \\
8 \\
\mathbf{4 6 . 8} \\
22.9 \\
48.8 \\
\end{array}$ & $\begin{array}{r}98.8 \\
60.6 \\
99.3 \\
98.5 \\
62.6 \\
115.0 \\
80.2 \\
52.8 \\
8 \\
\mathbf{8 3 . 5} \\
22.7 \\
27.2\end{array}$ & $\begin{array}{r}76.4 \\
46.0 \\
73.1 \\
72.4 \\
44.5 \\
104.7 \\
62.6 \\
46.0 \\
8 \\
\mathbf{6 5 . 7} \\
20.6 \\
31.4\end{array}$ & $\begin{array}{r}145.6 \\
202.5 \\
119.6 \\
167.3 \\
200.4 \\
171.1 \\
226.2 \\
121.6 \\
8 \\
\mathbf{1 6 9 . 3} \\
39.0 \\
23.0\end{array}$ & \begin{tabular}{r|}
120.8 \\
193.3 \\
106.1 \\
139.1 \\
185.6 \\
159.6 \\
217.6 \\
111.7 \\
8 \\
$\mathbf{1 5 4 . 2}$ \\
41.5 \\
26.9
\end{tabular} \\
\hline Southern Pine & SPIHMR & \begin{tabular}{r|}
1 \\
2 \\
3 \\
4 \\
5 \\
6 \\
\\
$\mathrm{n}$ \\
n \\
AVERAGE \\
STDEV \\
$\operatorname{COV}(\%)$
\end{tabular} & & & $\begin{array}{r}32.5 \\
18.7 \\
49.4 \\
26.2 \\
34.2 \\
9.0 \\
19.8 \\
33.1 \\
8 \\
\mathbf{2 7 . 9} \\
12.3 \\
44.1\end{array}$ & $\begin{array}{r}25.5 \\
13.7 \\
44.1 \\
22.0 \\
22.4 \\
4.6 \\
16.6 \\
25.4 \\
8 \\
21.8 \\
11.4 \\
52.4\end{array}$ & & & & \\
\hline
\end{tabular}


Appendix M. Strain energy release rate of treated southern pine bonded with PF and PVA adhesives.

\begin{tabular}{|c|c|c|c|c|c|c|}
\hline \multicolumn{3}{|c|}{ MAX $=$ Maximum, $\mathrm{ARR}=$ Arrested } & \multicolumn{4}{|c|}{ Strain Energy Release Rate (J/m2) } \\
\hline \multirow{2}{*}{$\begin{array}{c}\text { Wood } \\
\text { Species }\end{array}$} & \multirow{2}{*}{$\begin{array}{c}\text { Surface } \\
\text { Treatment }\end{array}$} & \multirow{2}{*}{$\begin{array}{l}\text { Number of } \\
\text { Replication }\end{array}$} & \multicolumn{2}{|c|}{ PVA } & \multicolumn{2}{|c|}{ PF } \\
\hline & & & MAX & ARR & MAX & ARR \\
\hline \multirow[t]{12}{*}{ Southern Pine } & \multirow[t]{12}{*}{ SPIXY } & 1 & 59.0 & 42.6 & 58.3 & 45.9 \\
\hline & & 2 & 130.1 & 100.2 & 25.4 & 19.4 \\
\hline & & 3 & 34.0 & 24.5 & 56.3 & 41.6 \\
\hline & & 4 & 49.3 & 49.8 & 49.3 & 43.6 \\
\hline & & 5 & 49.2 & 43.0 & 17.7 & 12.0 \\
\hline & & 6 & 76.2 & 64.2 & 61.2 & 54.3 \\
\hline & & & 59.6 & 46.0 & 64.5 & 43.8 \\
\hline & & & 76.7 & 68.6 & 86.9 & 71.8 \\
\hline & & & 8 & 8 & 8 & 8 \\
\hline & & AVERAGE & 66.7 & 54.9 & 52.5 & 41.5 \\
\hline & & STDEV & 29.3 & 22.8 & 22.1 & 18.8 \\
\hline & & $\operatorname{COV}(\%)$ & 43.9 & 41.6 & 42.0 & 45.2 \\
\hline \multirow[t]{12}{*}{ Southern Pine } & \multirow[t]{12}{*}{ SPINA } & 1 & 91.4 & 74.4 & 126.7 & 82.0 \\
\hline & & 2 & 77.9 & 67.6 & 155.6 & 144.5 \\
\hline & & 3 & 94.1 & 81.8 & 202.0 & 188.4 \\
\hline & & 4 & 59.7 & 50.3 & 154.2 & 142.6 \\
\hline & & 5 & 104.0 & 92.5 & 233.3 & 221.1 \\
\hline & & 6 & 165.8 & 128.3 & 244.2 & 221.4 \\
\hline & & & 241.8 & 209.8 & 261.0 & 238.4 \\
\hline & & & 155.5 & 105.9 & 148.5 & 134.7 \\
\hline & & & 8 & 8 & 8 & 8 \\
\hline & & AVERAGE & 123.8 & 101.3 & 190.7 & 171.6 \\
\hline & & STDEV & 60.0 & 49.9 & 51.0 & 54.3 \\
\hline & & $\operatorname{COV}(\%)$ & 48.5 & 49.3 & 26.7 & 31.6 \\
\hline \multirow[t]{12}{*}{ Southern Pine } & \multirow[t]{12}{*}{ SPIXYNA } & 1 & 52.3 & 45.5 & 220.9 & 197.9 \\
\hline & & 2 & 124.5 & 75.3 & 164.8 & 152.8 \\
\hline & & 3 & 84.2 & 71.3 & 182.6 & 161.4 \\
\hline & & 4 & 151.2 & 129.2 & 234.0 & 216.4 \\
\hline & & 5 & 48.5 & 32.2 & 134.4 & 121.0 \\
\hline & & 6 & 124.8 & 92.2 & 147.2 & 123.0 \\
\hline & & & 138.9 & 107.5 & 208.7 & 194.8 \\
\hline & & & 194.9 & 154.5 & 216.1 & 202.4 \\
\hline & & & & 8 & & \\
\hline & & AVERAGE & 114.9 & 88.5 & 188.6 & 171.2 \\
\hline & & STDEV & 50.4 & 41.2 & 36.9 & 37.0 \\
\hline & & $\operatorname{COV}(\%)$ & 43.8 & 46.6 & 19.5 & 21.6 \\
\hline
\end{tabular}




\section{Vita}

\section{Milan Šernek}

Milan Šernek was born and raised in Slovenia. He achieved the B.S. and M.S. degrees at the University of Ljubljana, Department of Wood Science, under the guidance of Dr. Jože Resnik. During his master's study, Milan met Dr. Frederick Kamke of Virginia Tech. Dr. Kamke invited Milan to work at Virginia Tech in 1997-98 as a visiting scientist. Milan started his doctoral study in the Department of Wood Science and Forest Products at Virginia Polytechnic Institute and State University in January 2000. His doctoral research, which was supervised by Dr. Wolfgang Glasser and Dr. Frederick Kamke, dealt with wood surface inactivation, wood wettability, wood adhesion, and wood-based composites. 